ET -172

United States Derartment of Agriculture

Bureau of Entomology and Plant Quarantine

\title{
PERCENTAGE TABLES \\ FOR NUMBERS FROM 1 TO 250
}

By Charles A. Clark,

Division of Cereal and Forage Insect Investigations

\section{INTRODUCTION}

Percentage tables similar to those made available in this publication have been used for several seasons at the entomological laboratories engaged in the study of the European corn borer in the United States. The tables have been found very useful in a number of different phases of the research progran on the corn borer, and, with the hope that they will prove useful to many research workers in various fields of irvestigation, it seems worth while to make them generally available. They have been carefully checked, and it is believed that their use will reduce calculation errors.

The use of these tables is quite simple, and the following example will suffice to show how they should be used.

Parasitization studies: Many collections of host insects have been made to obtain data on parasites and parasitization. One of these collections contained 137 host insects and three species of solitary parasites were reared thereirom. Ten adults of parasite A, 18 of parasite B, and 15 of parasite C, or a total of 43 parasites were recovered from the 137 host insects, and it is desired to know the percentage of parasitization by each parasite as well as by the parasite complex as a whole. Turning to the column 137 (the number of host insects in the collection) on page 25 of the tables, we may read the percentage of parasitization for each parasite at the point where the horizontal line opposite the proper figure for the number of parasites reared intersects the 137 column, thus:

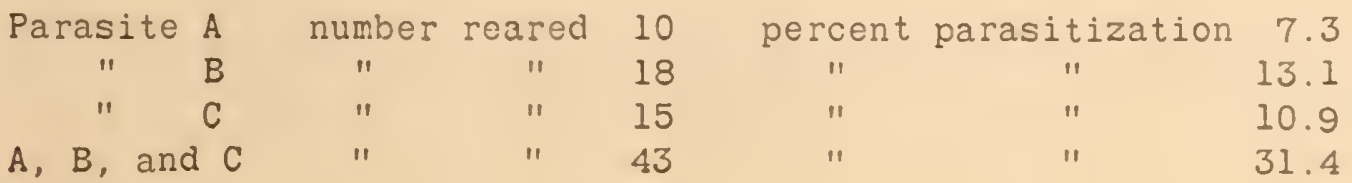


Parasitization percentages for the other collections may be determined in the same manner.

In many cases, as in the above example, it may not be considered necessary to record the percentages beyond the tenths place, but the percentage tables have been carried to the nearest thousandth for problems in which this degree of accuracy is desired.

The rounding procedure followed in these tables has been to add $I$ if the following digit is more than 5, to disregard the following digit if less than 5 , to add $I$ if the following digit is an even 5 and the digit to be rounded is odd, and to disregard the 5 if the digit to be rounded is even.

Often it will be found that the range of percentage calculations needed for a particular problem will be limited. Thus over 99 percent of the hundreds of collections of corn-borer larvae made annualiy to determine parasitization fall in the range 80 to 120 hosts collected, and parasitization, usually by several species, totals less than 50 percent. The thousands of percentage figures needed in this problem can all be read directly from only four pages $(12,14,16$, and 19) of the tables.

For more convenient use it is suggested that the staples be removed and the tables bound in a good looseleaf notebook cover with an ample series of fasterers. They can then be laid open flat on the desk at the pages particularly needed.

In setting up tables of this nature errors occur in spite of very careful checking. Every effort has been made to make the present tables accurate, but it is possible that a few errors occur. It will be appreciated if anyone finding an error in these tables will kindly inform the author. The author would also be interested in learnjing if similar tables for numbers above 250 would be useful to research workers. 
PLoopuos rume

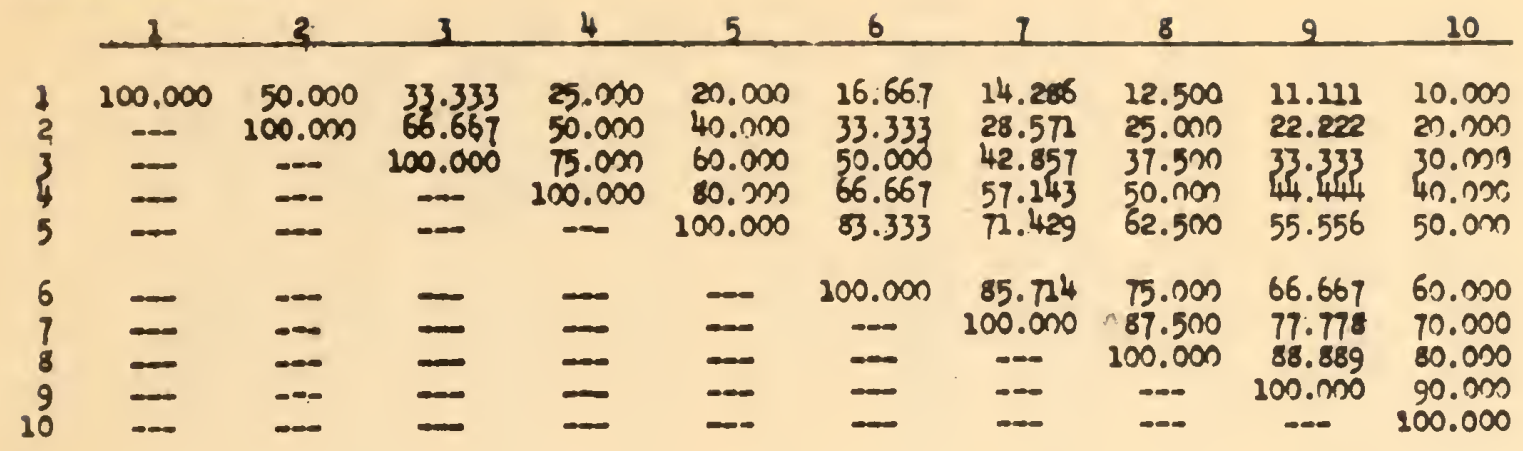


Digitized by the Internet Archive in 2013

http://archive.org/details/percentagetables00unit 


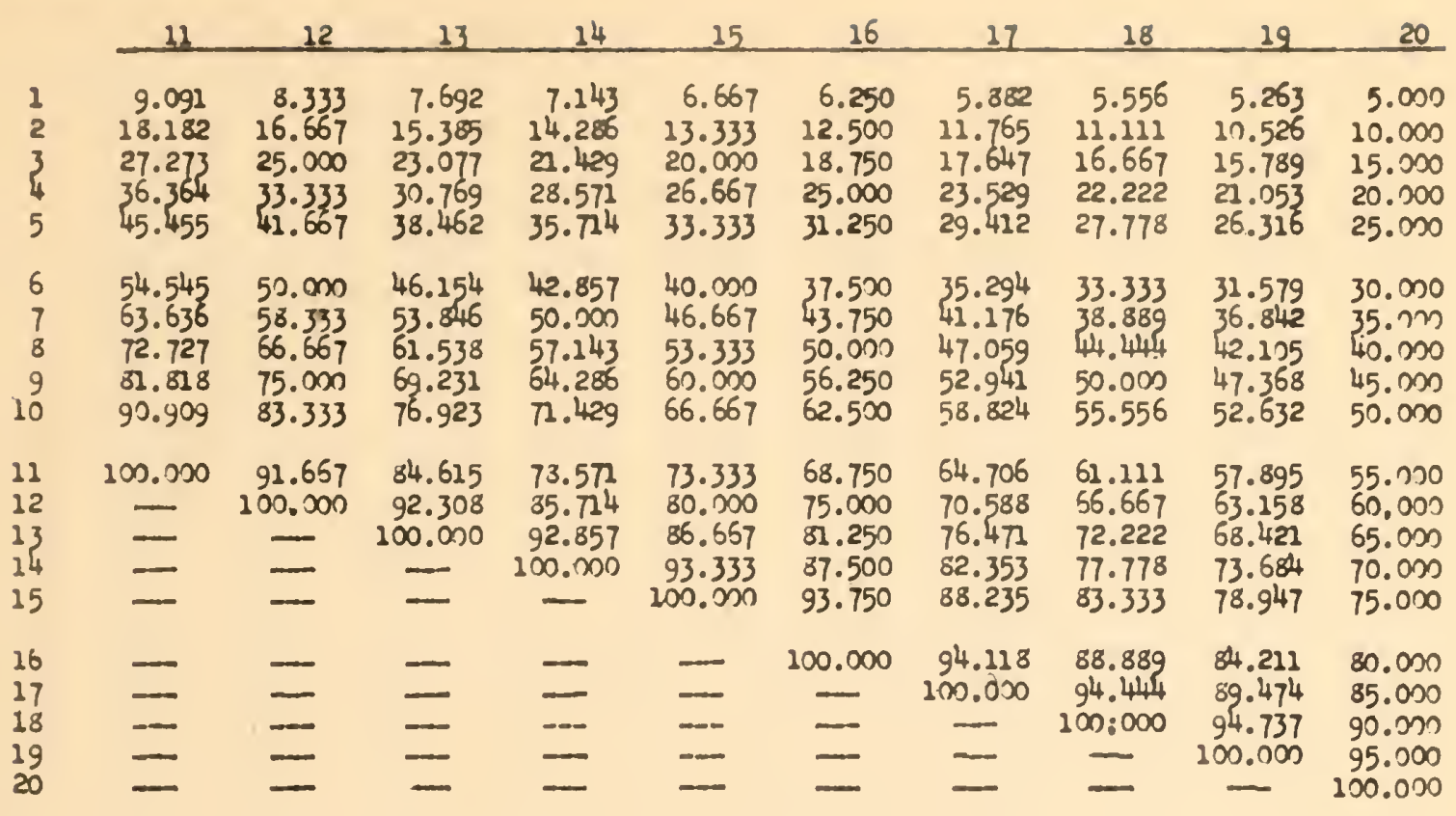




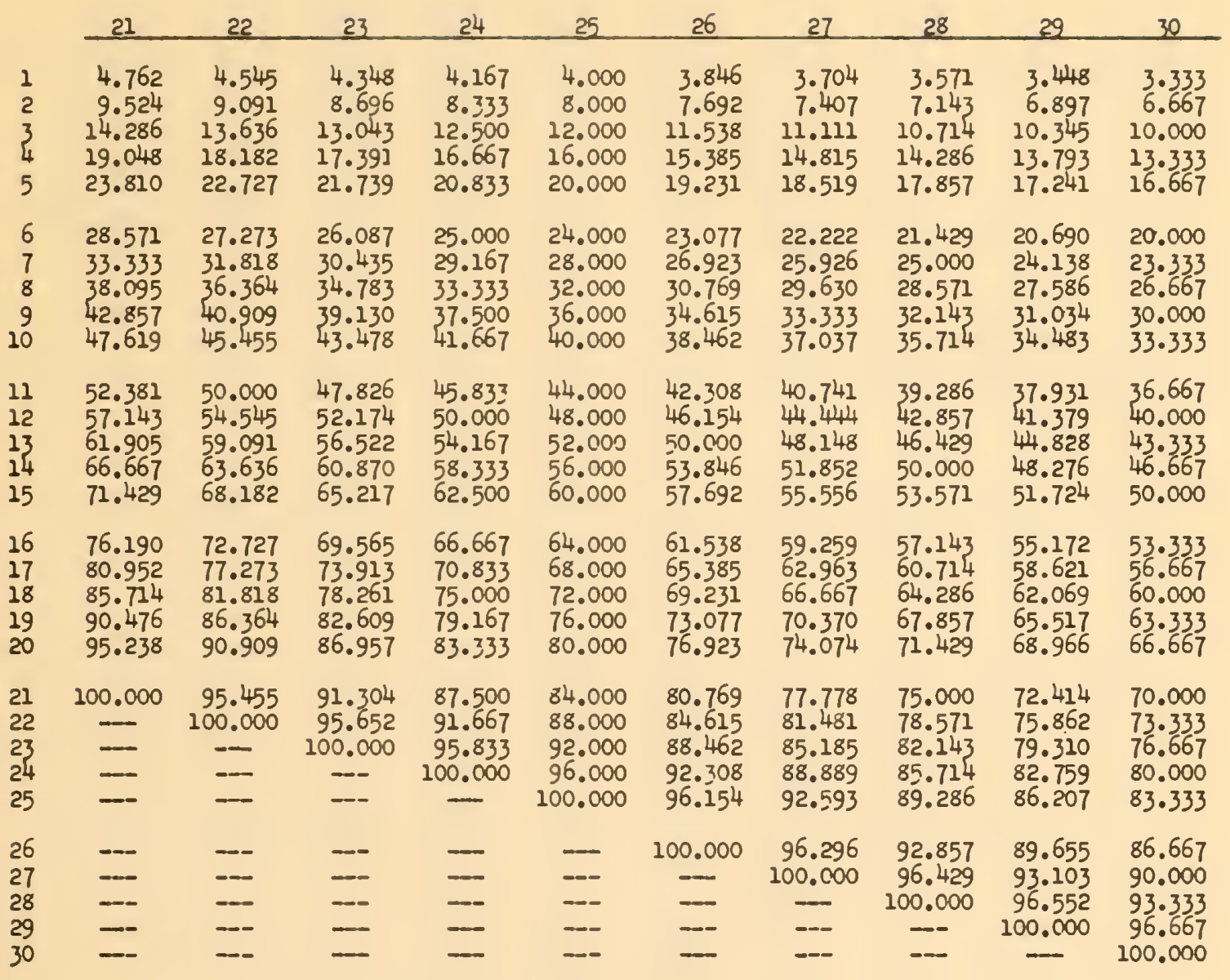




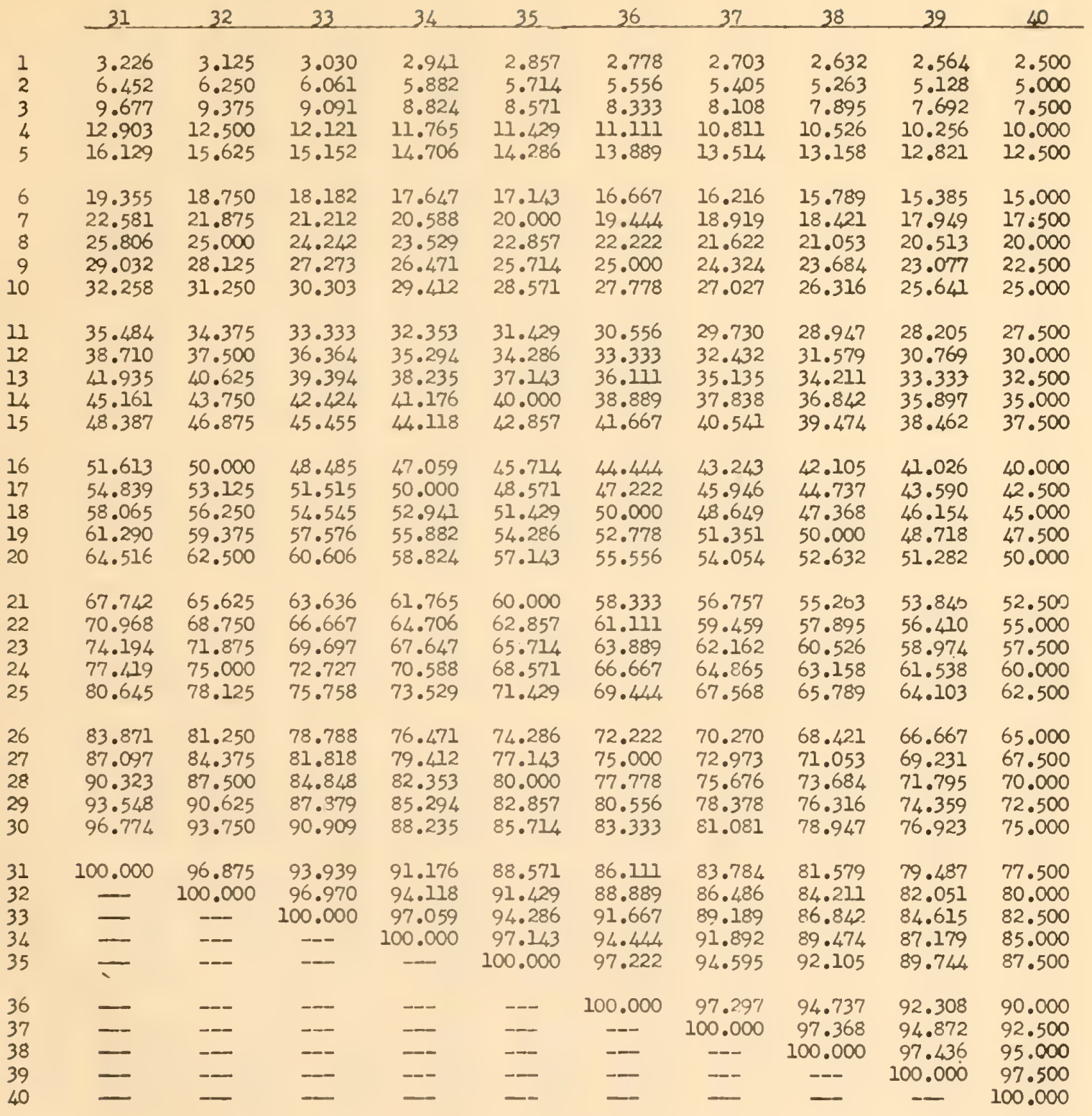





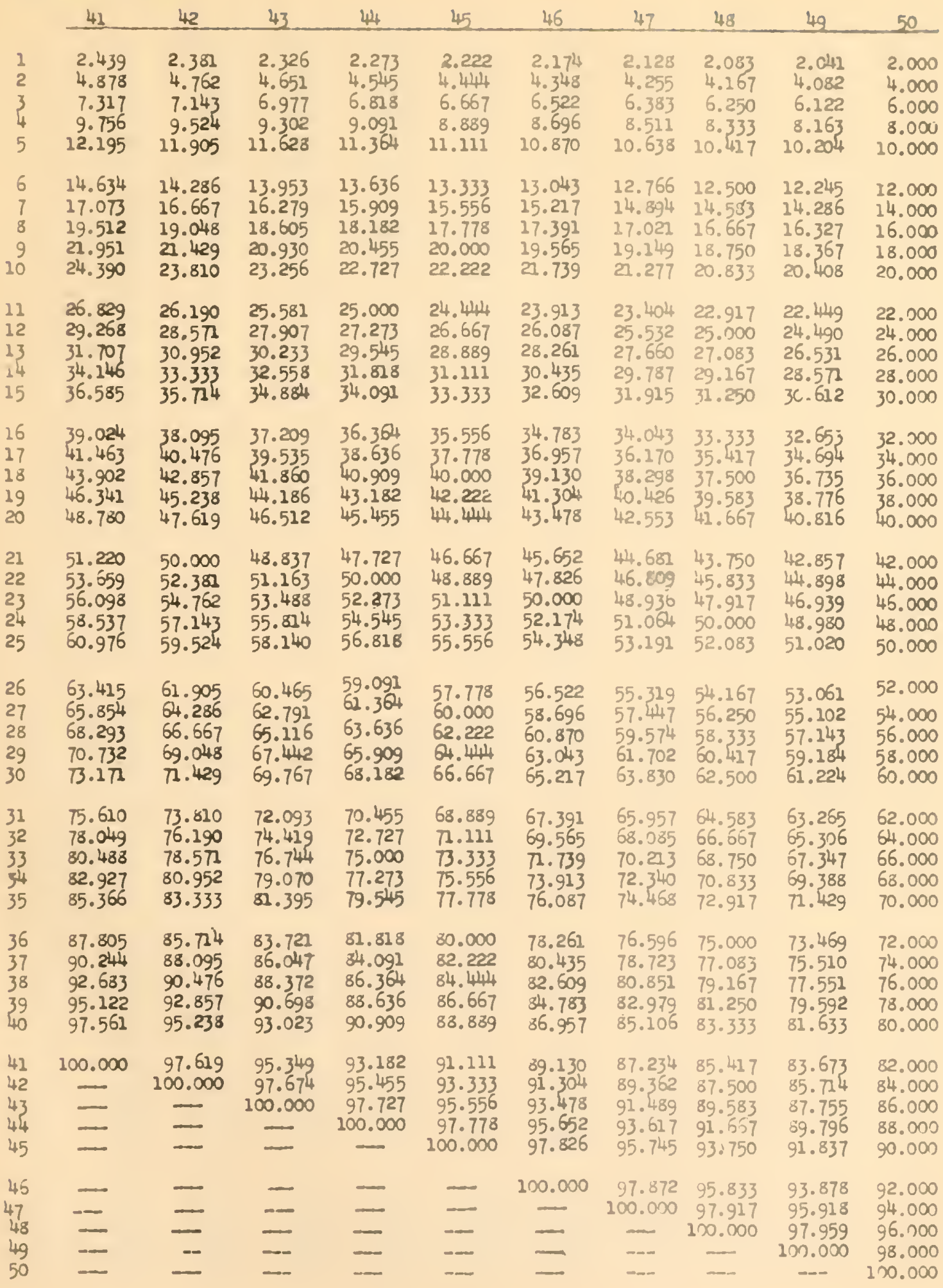





\begin{tabular}{|c|c|c|c|c|c|c|c|c|c|}
\hline 52 & 52 & 53 & 54 & 55 & 56 & 57 & 58 & 59 & 60 \\
\hline $\begin{array}{l}1.961 \\
3.922 \\
5.882 \\
7.843 \\
9.804\end{array}$ & $\begin{array}{l}1.923 \\
3.846 \\
5.769 \\
7.692 \\
9.615\end{array}$ & $\begin{array}{l}1.887 \\
3.774 \\
5.660 \\
7.547 \\
9.434\end{array}$ & $\begin{array}{l}1.852 \\
3.704 \\
5.556 \\
7.407 \\
9.259\end{array}$ & $\begin{array}{l}1.818 \\
5.656 \\
5.455 \\
7.273 \\
9.091\end{array}$ & $\begin{array}{l}1.786 \\
3.51 \\
5.357 \\
7.143 \\
8.929\end{array}$ & $\begin{array}{l}1.754 \\
3.509 \\
5.263 \\
7.018 \\
8.772\end{array}$ & $\begin{array}{l}1.724 \\
3.448 \\
5.172 \\
6.897 \\
8.621\end{array}$ & $\begin{array}{l}1.695 \\
3.390 \\
5.085 \\
6.780 \\
8.475\end{array}$ & $\begin{array}{l}1.667 \\
3.333 \\
5.000 \\
6.667 \\
8.333\end{array}$ \\
\hline $\begin{array}{l}11.765 \\
13.725 \\
15.686 \\
17.647 \\
19.608\end{array}$ & $\begin{array}{l}11.538 \\
13.462 \\
15.385 \\
17.308 \\
19.231\end{array}$ & $\begin{array}{l}11.324 \\
13.208 \\
15.094 \\
16.981 \\
18.868\end{array}$ & $\begin{array}{l}11.111 \\
12.963 \\
14.815 \\
16.667 \\
18.519\end{array}$ & $\begin{array}{l}10.909 \\
12.727 \\
14.545 \\
16.364 \\
18.182\end{array}$ & $\begin{array}{l}10.714 \\
12.500 \\
14.286 \\
16.012 \\
17.857\end{array}$ & $\begin{array}{l}10.526 \\
12.281 \\
14.035 \\
15.789 \\
17.544\end{array}$ & $\begin{array}{l}10.345 \\
12.069 \\
13.793 \\
15.517 \\
17.241\end{array}$ & $\begin{array}{l}10.169 \\
11.864 \\
13.559 \\
15.254 \\
16.949\end{array}$ & $\begin{array}{l}10.000 \\
11.667 \\
13.333 \\
15.000 \\
16.667\end{array}$ \\
\hline $\begin{array}{l}21.569 \\
23.529 \\
25.490 \\
27.451 \\
29.412\end{array}$ & $\begin{array}{l}21.154 \\
23.077 \\
25.000 \\
26.923 \\
28.846\end{array}$ & $\begin{array}{l}20.755 \\
22.642 \\
24.528 \\
26.425 \\
28.302\end{array}$ & $\begin{array}{l}20.370 \\
22.222 \\
24.074 \\
25.926 \\
27.778\end{array}$ & $\begin{array}{l}20.000 \\
21.818 \\
23.636 \\
25.455 \\
27.273\end{array}$ & $\begin{array}{l}19.643 \\
21.429 \\
23.214 \\
25.000 \\
26.786\end{array}$ & $\begin{array}{l}19.298 \\
21.053 \\
22.807 \\
24.561 \\
26.316\end{array}$ & $\begin{array}{l}18.966 \\
20.690 \\
22.414 \\
24.138 \\
25.862\end{array}$ & $\begin{array}{l}18.644 \\
20.339 \\
22.034 \\
23.729 \\
25.424\end{array}$ & $\begin{array}{l}18.333 \\
20.050 \\
21.667 \\
23.333 \\
25.000\end{array}$ \\
\hline $\begin{array}{l}31.373 \\
33.333 \\
35.294 \\
37.255 \\
39.216\end{array}$ & $\begin{array}{l}30.769 \\
32.692 \\
34.615 \\
36.538 \\
38.462\end{array}$ & $\begin{array}{l}30.189 \\
32.075 \\
33.962 \\
35.849 \\
37.736\end{array}$ & $\begin{array}{l}29.630 \\
32.481 \\
33.333 \\
35.185 \\
37.037\end{array}$ & $\begin{array}{l}29.091 \\
30.909 \\
32.727 \\
34.545 \\
36.364\end{array}$ & $\begin{array}{l}28.57 \\
30.357 \\
32.143 \\
33.929 \\
35.714\end{array}$ & $\begin{array}{l}28.070 \\
29.825 \\
31.579 \\
33.333 \\
35.088\end{array}$ & $\begin{array}{l}27.586 \\
29.310 \\
31.034 \\
32.759 \\
34.483\end{array}$ & $\begin{array}{l}27.119 \\
28.814 \\
30.508 \\
32.203 \\
33.898\end{array}$ & $\begin{array}{l}26.667 \\
28.333 \\
30.000 \\
31.667 \\
33.333\end{array}$ \\
\hline $\begin{array}{l}41.176 \\
43.137 \\
45.098 \\
47.059 \\
49.020\end{array}$ & $\begin{array}{l}40.385 \\
42.308 \\
44.231 \\
46.154 \\
48.077\end{array}$ & $\begin{array}{l}39.623 \\
41.509 \\
43.396 \\
45.283 \\
47.170\end{array}$ & $\begin{array}{l}38.889 \\
40: 741 \\
42.593 \\
44.444 \\
46.296\end{array}$ & $\begin{array}{l}38.182 \\
40.000 \\
41.818 \\
43.636 \\
45.455\end{array}$ & $\begin{array}{l}37.500 \\
39.286 \\
41.07 \\
42.857 \\
44.643\end{array}$ & $\begin{array}{l}36.842 \\
38.596 \\
40.351 \\
42.105 \\
43.860\end{array}$ & $\begin{array}{l}36.207 \\
37.931 \\
39.655 \\
41.379 \\
43.103\end{array}$ & $\begin{array}{l}35.593 \\
37.288 \\
38.983 \\
40.678 \\
42.373\end{array}$ & $\begin{array}{l}35.000 \\
36.667 \\
38.333 \\
40.000 \\
41.667\end{array}$ \\
\hline $\begin{array}{l}50.980 \\
52.941 \\
54.902 \\
56.863 \\
58.824\end{array}$ & $\begin{array}{l}50.000 \\
51.923 \\
53.846 \\
55.769 \\
57.692\end{array}$ & $\begin{array}{l}49.057 \\
50.943 \\
52.830 \\
54.727 \\
56.604\end{array}$ & $\begin{array}{l}48.148 \\
50.000 \\
51.852 \\
53.704 \\
55.556\end{array}$ & $\begin{array}{l}47.273 \\
49.091 \\
50.909 \\
52.727 \\
54.545\end{array}$ & $\begin{array}{l}46.429 \\
48.214 \\
50.000 \\
51.786 \\
53.571\end{array}$ & $\begin{array}{l}45.614 \\
47.368 \\
49.123 \\
50.877 \\
52.632\end{array}$ & $\begin{array}{l}44.828 \\
46.552 \\
48.276 \\
50.000 \\
51.724\end{array}$ & $\begin{array}{l}44.068 \\
45.763 \\
47.458 \\
49.153 \\
50.847\end{array}$ & $\begin{array}{l}43.333 \\
45.000 \\
46.667 \\
48.333 \\
50.000\end{array}$ \\
\hline $\begin{array}{l}60.724 \\
62.745 \\
64.706 \\
66.667 \\
68.627\end{array}$ & $\begin{array}{l}59.615 \\
61.538 \\
63.462 \\
65.385 \\
67.308\end{array}$ & $\begin{array}{l}58.491 \\
60.377 \\
62.264 \\
64.151 \\
66.038\end{array}$ & $\begin{array}{l}57.407 \\
59.259 \\
61.111 \\
62.963 \\
64.815\end{array}$ & $\begin{array}{l}56.364 \\
58.182 \\
60.000 \\
61.818 \\
63.636\end{array}$ & $\begin{array}{l}55.357 \\
57.143 \\
58.929 \\
50.714 \\
62.500\end{array}$ & $\begin{array}{l}54.386 \\
56.140 \\
57.895 \\
59.649 \\
61.404\end{array}$ & $\begin{array}{l}53.448 \\
55.172 \\
56.897 \\
58.621 \\
50.345\end{array}$ & $\begin{array}{l}52.542 \\
54.237 \\
55.932 \\
57.627 \\
59.322\end{array}$ & $\begin{array}{l}51.667 \\
53.333 \\
55.000 \\
56.657 \\
58.333\end{array}$ \\
\hline $\begin{array}{l}70.588 \\
72.549 \\
74.510 \\
76.471 \\
73.431\end{array}$ & $\begin{array}{l}69.231 \\
71.154 \\
73.077 \\
75.000 \\
76.923\end{array}$ & $\begin{array}{l}67.925 \\
69.811 \\
71.698 \\
73.585 \\
75.472\end{array}$ & $\begin{array}{l}66.667 \\
68.519 \\
70.370 \\
72.222 \\
74.074\end{array}$ & $\begin{array}{l}65.455 \\
67.273 \\
69.091 \\
70.909 \\
72.727\end{array}$ & $\begin{array}{l}64.285 \\
66.071 \\
67.857 \\
69.643 \\
71.429\end{array}$ & $\begin{array}{l}63.158 \\
64.912 \\
66.667 \\
68.421 \\
70.175\end{array}$ & $\begin{array}{l}62.069 \\
63.793 \\
65.517 \\
67.241 \\
68.966\end{array}$ & $\begin{array}{l}61.017 \\
62.712 \\
54.407 \\
66.102 \\
57.797\end{array}$ & $\begin{array}{l}60.000 \\
61.667 \\
63.333 \\
65.000 \\
66.667\end{array}$ \\
\hline $\begin{array}{l}80.392 \\
82.353 \\
84.314 \\
86.275 \\
88.235\end{array}$ & $\begin{array}{l}78.846 \\
80.769 \\
82.692 \\
84.615 \\
86.538\end{array}$ & $\begin{array}{l}77.358 \\
79.245 \\
81.132 \\
83.019 \\
84.906\end{array}$ & $\begin{array}{l}75.926 \\
77.778 \\
79.630 \\
81.481 \\
83.333\end{array}$ & $\begin{array}{l}74.545 \\
76.364 \\
78.182 \\
80.000 \\
81.818\end{array}$ & $\begin{array}{l}73.214 \\
75.000 \\
76.786 \\
78.57 \\
80.357\end{array}$ & $\begin{array}{l}71.930 \\
73.684 \\
75.439 \\
77.193 \\
78.947\end{array}$ & $\begin{array}{l}70.690 \\
72.414 \\
74.138 \\
75.862 \\
77.586\end{array}$ & $\begin{array}{l}69.492 \\
71.186 \\
72.881 \\
74.576 \\
76.271\end{array}$ & $\begin{array}{l}68.333 \\
70.000 \\
71.667 \\
73.333 \\
75.000\end{array}$ \\
\hline $\begin{array}{l}90.196 \\
92.157 \\
94.118 \\
96.078 \\
98.039\end{array}$ & $\begin{array}{l}88.462 \\
90.385 \\
92.308 \\
94.231 \\
96.154\end{array}$ & $\begin{array}{l}86.792 \\
86.679 \\
90.560 \\
92.453 \\
94.340\end{array}$ & $\begin{array}{l}85.185 \\
87.037 \\
88.889 \\
90.741 \\
92.593\end{array}$ & $\begin{array}{l}83.636 \\
85.455 \\
87.273 \\
89.091 \\
90.909\end{array}$ & $\begin{array}{l}82.143 \\
83.929 \\
85.714 \\
87.500 \\
89.286\end{array}$ & $\begin{array}{l}80.702 \\
82.456 \\
84.211 \\
85.965 \\
87.719\end{array}$ & $\begin{array}{l}79.320 \\
81.034 \\
82.759 \\
84.483 \\
86.207\end{array}$ & $\begin{array}{l}77.966 \\
79.651 \\
81.356 \\
83.051 \\
84.746\end{array}$ & $\begin{array}{l}76.667 \\
78.333 \\
80.000 \\
81.667 \\
83.333\end{array}$ \\
\hline
\end{tabular}



PERCEITAGE TABLES

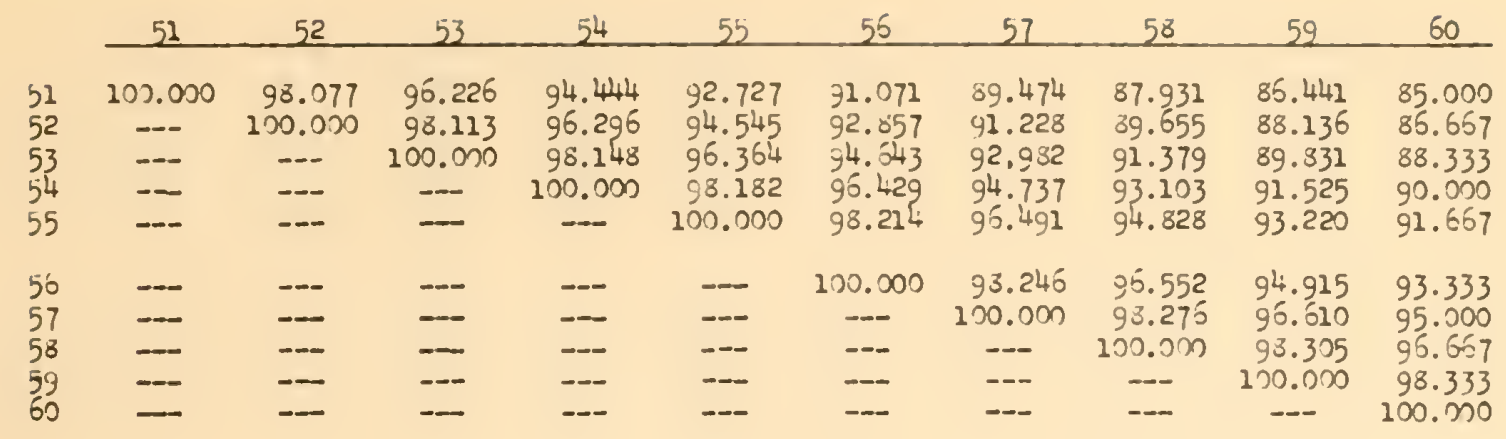





\begin{tabular}{|c|c|c|c|c|c|c|c|c|c|}
\hline 61 & 62 & 63 & 64 & 65 & 66 & 67 & 68 & 69 & 70 \\
\hline $\begin{array}{l}1.639 \\
3.279 \\
4.918 \\
6.557 \\
8.197\end{array}$ & $\begin{array}{l}1.613 \\
3.226 \\
4.839 \\
6.452 \\
8.065\end{array}$ & $\begin{array}{l}1.587 \\
3.175 \\
4.762 \\
6.349 \\
7.937\end{array}$ & $\begin{array}{l}1.562 \\
3.125 \\
4.688 \\
6.250 \\
7.812\end{array}$ & $\begin{array}{l}1.538 \\
3.077 \\
4.615 \\
6.154 \\
7.692\end{array}$ & $\begin{array}{l}1.515 \\
3.030 \\
4.545 \\
6.061 \\
7.576\end{array}$ & $\begin{array}{l}1.493 \\
2.985 \\
4.478 \\
5.970 \\
7.463\end{array}$ & $\begin{array}{l}1.471 \\
2.941 \\
4.412 \\
5.882 \\
7.353\end{array}$ & $\begin{array}{l}1.449 \\
2.899 \\
4.348 \\
5.797 \\
7.246\end{array}$ & $\begin{array}{l}1.429 \\
2.857 \\
4.286 \\
5.714 \\
7.143\end{array}$ \\
\hline $\begin{array}{r}9.836 \\
11.475 \\
13.115 \\
14.754 \\
16.393\end{array}$ & $\begin{array}{r}9.677 \\
11.290 \\
7 . .903 \\
1 . .516 \\
16.129\end{array}$ & $\begin{array}{r}9.524 \\
11.111 \\
12.698 \\
14.286 \\
15.873\end{array}$ & $\begin{array}{r}9.375 \\
10.8 \\
12 \cdot: 00 \\
14.062 \\
15.625\end{array}$ & $\begin{array}{r}9.231 \\
10.769 \\
12.308 \\
13.846 \\
15.385\end{array}$ & $\begin{array}{r}9.091 \\
10.606 \\
12.121 \\
13.636 \\
15.152\end{array}$ & $\begin{array}{r}8.955 \\
10.448 \\
11.940 \\
13.433 \\
14.925\end{array}$ & $\begin{array}{r}8.824 \\
10.294 \\
11.765 \\
13.235 \\
14.706\end{array}$ & $\begin{array}{r}8.696 \\
10.145 \\
11.594 \\
13.043 \\
14.493\end{array}$ & $\begin{array}{r}8.571 \\
10.000 \\
11.429 \\
12.857 \\
14.286\end{array}$ \\
\hline $\begin{array}{l}18.033 \\
19.672 \\
21.311 \\
22.951 \\
24.590\end{array}$ & $\begin{array}{l}17.742 \\
19.355 \\
20.968 \\
22.581 \\
24.194\end{array}$ & $\begin{array}{l}17.460 \\
19.048 \\
20.635 \\
22.222 \\
23.810\end{array}$ & $\begin{array}{l}17.188 \\
18.750 \\
20.312 \\
21.875 \\
23.438\end{array}$ & $\begin{array}{l}16.923 \\
18.462 \\
20.000 \\
21.538 \\
23.077\end{array}$ & $\begin{array}{l}16.667 \\
18.182 \\
19.697 \\
21.212 \\
22.727\end{array}$ & $\begin{array}{l}16.418 \\
17.910 \\
19.403 \\
20.896 \\
22.388\end{array}$ & $\begin{array}{l}16.176 \\
17.647 \\
19.118 \\
20.588 \\
22.059\end{array}$ & $\begin{array}{l}15.942 \\
17.391 \\
18.841 \\
20.290 \\
21.739\end{array}$ & $\begin{array}{l}15.714 \\
17.143 \\
18.571 \\
20.000 \\
21.429\end{array}$ \\
\hline $\begin{array}{l}26.230 \\
27.869 \\
29.508 \\
31.148 \\
32.787\end{array}$ & $\begin{array}{l}25.806 \\
27.419 \\
29.032 \\
30.645 \\
32.258\end{array}$ & $\begin{array}{l}25.397 \\
26.984 \\
28.571 \\
30.159 \\
31.746\end{array}$ & $\begin{array}{l}25.000 \\
26.562 \\
28.125 \\
29.688 \\
31.250\end{array}$ & $\begin{array}{l}24.615 \\
26.154 \\
27.692 \\
29.231 \\
30.769\end{array}$ & $\begin{array}{l}24.242 \\
25.758 \\
27.273 \\
28.788 \\
30.303\end{array}$ & $\begin{array}{l}23.881 \\
25.373 \\
26.866 \\
28.358 \\
29.851\end{array}$ & $\begin{array}{l}23.529 \\
25.000 \\
26.471 \\
27.941 \\
29.412\end{array}$ & $\begin{array}{l}23.188 \\
24.638 \\
26.087 \\
27.536 \\
28.986\end{array}$ & $\begin{array}{l}22.857 \\
24.286 \\
25.714 \\
27.143 \\
28.571\end{array}$ \\
\hline $\begin{array}{l}34.426 \\
36.066 \\
37.705 \\
39.344 \\
40.984\end{array}$ & $\begin{array}{l}33.871 \\
35.484 \\
37.097 \\
38.710 \\
40.323\end{array}$ & $\begin{array}{l}33.333 \\
34.921 \\
36.508 \\
38.095 \\
39.683\end{array}$ & $\begin{array}{l}32.812 \\
34.375 \\
35.938 \\
37.500 \\
39.062\end{array}$ & $\begin{array}{l}32.308 \\
33.846 \\
35.385 \\
36.923 \\
38.462\end{array}$ & $\begin{array}{l}31.818 \\
33.333 \\
34.848 \\
36.364 \\
37.879\end{array}$ & $\begin{array}{l}31.343 \\
32.836 \\
34.328 \\
35.821 \\
37.313\end{array}$ & $\begin{array}{l}30.882 \\
32.353 \\
33.824 \\
35.294 \\
36.765\end{array}$ & $\begin{array}{l}30.435 \\
31.884 \\
33.333 \\
34.783 \\
36.232\end{array}$ & $\begin{array}{l}30.000 \\
31.429 \\
.32 .857 \\
34.286 \\
35.714\end{array}$ \\
\hline $\begin{array}{l}42.623 \\
44.262 \\
45.902 \\
47.541 \\
49.180\end{array}$ & $\begin{array}{l}41.935 \\
43.548 \\
45.161 \\
46.774 \\
48.387\end{array}$ & $\begin{array}{l}47.270 \\
42.857 \\
44.444 \\
46.032 \\
47.619\end{array}$ & $\begin{array}{l}40.625 \\
42.188 \\
43.750 \\
45.312 \\
46.875\end{array}$ & $\begin{array}{l}40.000 \\
41.538 \\
43.077 \\
44.615 \\
46.154\end{array}$ & $\begin{array}{l}39.394 \\
40.909 \\
42.424 \\
43.939 \\
45.455\end{array}$ & $\begin{array}{l}38.806 \\
40.298 \\
41.791 \\
43.284 \\
44.776\end{array}$ & $\begin{array}{l}38.235 \\
39.706 \\
41.176 \\
42.647 \\
44.118\end{array}$ & $\begin{array}{l}37.681 \\
39.130 \\
40.580 \\
42.029 \\
43.478\end{array}$ & $\begin{array}{l}37.143 \\
38.571 \\
40.000 \\
41.429 \\
42.857\end{array}$ \\
\hline $\begin{array}{l}50.820 \\
52.459 \\
54.098 \\
55.738 \\
57.377\end{array}$ & $\begin{array}{l}50.000 \\
51.613 \\
53.226 \\
54.839 \\
56.452\end{array}$ & $\begin{array}{l}49.206 \\
50.794 \\
52.381 \\
53.968 \\
55.556\end{array}$ & $\begin{array}{l}48.438 \\
50.000 \\
51.562 \\
53.125 \\
54.688\end{array}$ & $\begin{array}{l}47.692 \\
49.231 \\
50.769 \\
52.308 \\
53.846\end{array}$ & $\begin{array}{l}46.970 \\
48.485 \\
50.000 \\
51.515 \\
53.030\end{array}$ & $\begin{array}{l}46.269 \\
47.761 \\
49.254 \\
50.746 \\
52.239\end{array}$ & $\begin{array}{l}45.588 \\
47.059 \\
48.529 \\
50.000 \\
51.471\end{array}$ & $\begin{array}{l}44.928 \\
46.377 \\
47.826 \\
49.275 \\
50.725\end{array}$ & $\begin{array}{l}44.286 \\
45.714 \\
47.143 \\
48.571 \\
50.000\end{array}$ \\
\hline $\begin{array}{l}59.016 \\
60.656 \\
62.295 \\
63.934 \\
65.574\end{array}$ & $\begin{array}{l}58.065 \\
59.677 \\
61.290 \\
62.903 \\
64.516\end{array}$ & $\begin{array}{l}57.143 \\
58.730 \\
60.317 \\
61.905 \\
63.492\end{array}$ & $\begin{array}{l}56.250 \\
57.812 \\
59.375 \\
60.938 \\
62.500\end{array}$ & $\begin{array}{l}55.385 \\
56.923 \\
58.462 \\
60.000 \\
61.538\end{array}$ & $\begin{array}{l}54.545 \\
56.061 \\
57.576 \\
59.091 \\
60.606\end{array}$ & $\begin{array}{l}53.731 \\
55.224 \\
56.716 \\
58.209 \\
59.701\end{array}$ & $\begin{array}{l}52.947 \\
54.412 \\
55.882 \\
57.353 \\
58.824\end{array}$ & $\begin{array}{l}52.174 \\
53.623 \\
55.072 \\
56.522 \\
57.971\end{array}$ & $\begin{array}{l}51.429 \\
52.857 \\
54.286 \\
55.714 \\
57.143\end{array}$ \\
\hline $\begin{array}{l}67.213 \\
68.852 \\
70.492 \\
72.131 \\
73.770\end{array}$ & $\begin{array}{l}66.129 \\
67.742 \\
69.355 \\
70.968 \\
72.581\end{array}$ & $\begin{array}{l}65.079 \\
66.667 \\
68.254 \\
69.841 \\
71.429\end{array}$ & $\begin{array}{l}64.062 \\
65.625 \\
67.188 \\
68.750 \\
70.312\end{array}$ & $\begin{array}{l}63.077 \\
64.615 \\
66.154 \\
67.692 \\
69.231\end{array}$ & $\begin{array}{l}52.121 \\
63.636 \\
65.152 \\
66.667 \\
68.182\end{array}$ & $\begin{array}{l}61.194 \\
62.687 \\
64.179 \\
65.672 \\
67.164\end{array}$ & $\begin{array}{l}60.294 \\
61.765 \\
63.235 \\
64.706 \\
66.176\end{array}$ & $\begin{array}{l}59.424 \\
60.870 \\
62.319 \\
63.768 \\
65.217\end{array}$ & $\begin{array}{l}58.571 \\
60.000 \\
61.429 \\
62.857 \\
64.286\end{array}$ \\
\hline $\begin{array}{l}75.410 \\
77.049 \\
78.689 \\
80.328 \\
81.967\end{array}$ & $\begin{array}{l}74.194 \\
75.806 \\
77.419 \\
79.032 \\
80.645\end{array}$ & $\begin{array}{l}73.016 \\
74.603 \\
76.190 \\
77.778 \\
79.365\end{array}$ & $\begin{array}{l}71.875 \\
73.438 \\
75.000 \\
76.562 \\
78.125\end{array}$ & $\begin{array}{l}70.769 \\
72.308 \\
73.846 \\
75.385 \\
76.923\end{array}$ & $\begin{array}{l}69.697 \\
71.212 \\
72.727 \\
74.242 \\
75.758\end{array}$ & $\begin{array}{l}68.657 \\
70.149 \\
71.642 \\
73.134 \\
74.627\end{array}$ & $\begin{array}{l}67.647 \\
69.118 \\
70.588 \\
72.059 \\
73.529\end{array}$ & $\begin{array}{l}66.667 \\
68.116 \\
69.565 \\
71.014 \\
72.464\end{array}$ & $\begin{array}{l}65.714 \\
67.143 \\
68.571 \\
70.000 \\
71.429\end{array}$ \\
\hline
\end{tabular}



PERCENTAGE TABLES

\begin{tabular}{|c|c|c|c|c|c|c|c|c|c|}
\hline 61 & 62 & 63 & 64 & 65 & 66 & 67 & 68 & 69 & 20 \\
\hline 83.607 & 82.258 & 80.952 & 79.688 & 78.462 & 77.273 & 76.119 & 75.000 & 73.913 & 72.857 \\
\hline & 83.87 & & & 80.000 & & & 76.47 & & 74,286 \\
\hline .885 & 85.484 & 84.127 & 82.812 & 81.538 & 80.3 & & 77.942 & 76.812 & 75.74 \\
\hline .525 & 87.097 & 85.714 & 84.375 & 83.077 & 81.818 & 80.597 & 79.422 & 78.261 & 7.143 \\
\hline 90.16 & 88.710 & 87.302 & 85.938 & 84.615 & 83. & 82.090 & 80.882 & 79.70 & 78.571 \\
\hline .803 & 90.323 & 88.889 & 87.500 & 86.154 & 84.848 & 83.582 & 82.353 & 81.159 & 80.000 \\
\hline & 91.935 & & 89. & & 86. & & 83.824 & & 81.429 \\
\hline 95.082 & 93.548 & 92.063 & 90.625 & 89.231 & 87.879 & 86.567 & 85.294 & 84. & 82.857 \\
\hline 96.721 & 95.161 & 93.651 & 92.188 & 90.769 & 89.3 & 88.060 & 86.765 & 85.507 & 84.286 \\
\hline 98.361 & 96.774 & 95.238 & 93.750 & 92.308 & 90.909 & 89.552 & 88.235 & 86.957 & 85.714 \\
\hline 00.000 & 98.387 & 96.825 & 95.312 & 93.846 & 92.424 & 91.045 & 89.706 & $88.406^{\circ}$ & 87.143 \\
\hline & 100.000 & 98.413 & 96.875 & 95.385 & 93.939 & 92.537 & 91.176 & 89.855 & 88.571 \\
\hline - & - & 100.000 & 98.438 & 96.923 & 95.4 & 94.030 & 92.647 & 91.304 & 90.000 \\
\hline & $\ldots$ & - & 100.000 & 98.462 & 96.970 & 95.522 & 94.118 & 92.754 & 91.429 \\
\hline & - & & & 100.000 & 98.485 & 97.015 & 95.588 & 94.203 & 92.857 \\
\hline & - & - & - & - & 100.000 & 98.507 & 97.059 & 95.652 & 94.286 \\
\hline & - & & - & - & - & 100.000 & & 97.101 & 95.714 \\
\hline & - & - & - & - & - & - & 100.000 & 98.551 & 97.143 \\
\hline & - & $\ldots$ & $\ldots$ & - & $\ldots$ & - & --- & 100.000 & 98.571 \\
\hline & $\ldots$ & - & $\ldots$ & - & --- & $\ldots$ & -- & -- & 100.000 \\
\hline
\end{tabular}





\begin{tabular}{|c|c|c|c|c|c|c|c|c|c|c|}
\hline & 71 & 72 & 73 & 74 & 75 & 76 & 77 & 78 & 79 & 80 \\
\hline $\begin{array}{l}1 \\
2 \\
3 \\
4 \\
5\end{array}$ & $\begin{array}{l}1.408 \\
2.817 \\
4.225 \\
5.634 \\
7.042\end{array}$ & $\begin{array}{l}1.389 \\
2.778 \\
4.167 \\
5.556 \\
6.944\end{array}$ & $\begin{array}{l}1.370 \\
2.740 \\
4.110 \\
5.479 \\
6.849\end{array}$ & $\begin{array}{l}1.351 \\
2.703 \\
4.054 \\
5.405 \\
6.757\end{array}$ & $\begin{array}{l}1.333 \\
2.667 \\
4.000 \\
5.333 \\
6.667\end{array}$ & $\begin{array}{l}1.316 \\
2.632 \\
3.947 \\
5.263 \\
6.579\end{array}$ & $\begin{array}{l}1.299 \\
2.597 \\
3.896 \\
5.195 \\
6.494\end{array}$ & $\begin{array}{l}1.282 \\
2.564 \\
3.81,6 \\
5.128 \\
6.410\end{array}$ & $\begin{array}{l}1.266 \\
2.532 \\
3.797 \\
5.063 \\
6.329\end{array}$ & $\begin{array}{l}1.250 \\
2.500 \\
3.750 \\
5.000 \\
6.250\end{array}$ \\
\hline $\begin{array}{r}6 \\
7 \\
8 \\
9 \\
10\end{array}$ & $\begin{array}{r}8.451 \\
9.859 \\
11.268 \\
12.676 \\
14.085\end{array}$ & $\begin{array}{r}8.333 \\
9.722 \\
11.111 \\
12.500 \\
13.889\end{array}$ & $\begin{array}{r}8.219 \\
9.589 \\
10.959 \\
12.329 \\
13.699\end{array}$ & $\begin{array}{r}\therefore .108 \\
9.459 \\
10.811 \\
12.162 \\
13.514\end{array}$ & $\begin{array}{r}8.000 \\
9.333 \\
10.667 \\
12.000 \\
13.333\end{array}$ & $\begin{array}{r}7.895 \\
9.211 \\
10.526 \\
11.842 \\
13.158\end{array}$ & $\begin{array}{r}7.792 \\
9.091 \\
10.390 \\
11.688 \\
12.987\end{array}$ & $\begin{array}{r}7.692 \\
8.974 \\
10.256 \\
11.538 \\
12.821\end{array}$ & $\begin{array}{r}7.595 \\
8.861 \\
10.127 \\
11.392 \\
12.658\end{array}$ & $\begin{array}{r}7.500 \\
8.750 \\
10.000 \\
11.250 \\
12.500\end{array}$ \\
\hline $\begin{array}{l}11 \\
12 \\
13 \\
14 \\
15\end{array}$ & $\begin{array}{l}15.493 \\
16.901 \\
18.310 \\
19.718 \\
21.127\end{array}$ & $\begin{array}{l}15.278 \\
16.667 \\
18.056 \\
19.444 \\
20.833\end{array}$ & $\begin{array}{l}15.069 \\
16.438 \\
17.808 \\
19.178 \\
20.548\end{array}$ & $\begin{array}{l}14.865 \\
16.216 \\
17.568 \\
18.919 \\
20.270\end{array}$ & $\begin{array}{l}14.667 \\
16.000 \\
17.333 \\
18.667 \\
20.000\end{array}$ & $\begin{array}{l}14.474 \\
15.789 \\
17.105 \\
18.421 \\
19.737\end{array}$ & $\begin{array}{l}14.286 \\
15.584 \\
16.883 \\
18.182 \\
19.481\end{array}$ & $\begin{array}{l}14.103 \\
15.385 \\
16.667 \\
17.949 \\
19.231\end{array}$ & $\begin{array}{l}13.924 \\
15.190 \\
16.456 \\
17.722 \\
18.987\end{array}$ & $\begin{array}{l}13.750 \\
15.000 \\
16.250 \\
17.500 \\
18.750\end{array}$ \\
\hline $\begin{array}{l}16 \\
17 \\
18 \\
19 \\
20\end{array}$ & $\begin{array}{l}22.535 \\
23.944 \\
25.352 \\
26.761 \\
28.169\end{array}$ & $\begin{array}{l}22.222 \\
23.611 \\
25.000 \\
26.389 \\
27.778\end{array}$ & $\begin{array}{l}21.918 \\
23.288 \\
24.658 \\
26.027 \\
27.397\end{array}$ & $\begin{array}{l}21.622 \\
22.973 \\
24.324 \\
25.676 \\
27.027\end{array}$ & $\begin{array}{l}21.333 \\
22.667 \\
24.000 \\
25.333 \\
26.667\end{array}$ & $\begin{array}{l}21.053 \\
22.368 \\
23.684 \\
25.000 \\
26.316\end{array}$ & $\begin{array}{l}20.779 \\
22.078 \\
23.377 \\
24.675 \\
25.974\end{array}$ & $\begin{array}{l}20.513 \\
21.795 \\
23.077 \\
24.359 \\
25.641\end{array}$ & $\begin{array}{l}20.253 \\
21.519 \\
22.785 \\
24.051 \\
25.316\end{array}$ & $\begin{array}{l}20.000 \\
21.250 \\
22.500 \\
23.750 \\
25.000\end{array}$ \\
\hline $\begin{array}{l}21 \\
22 \\
23 \\
24 \\
25\end{array}$ & $\begin{array}{l}29.577 \\
30.986 \\
32.394 \\
33.803 \\
35.211\end{array}$ & $\begin{array}{l}29.167 \\
30.556 \\
31.944 \\
33.333 \\
34.722\end{array}$ & $\begin{array}{l}28.767 \\
30.137 \\
31.507 \\
32.877 \\
34.247\end{array}$ & $\begin{array}{l}28.378 \\
29.730 \\
31.081 \\
32.432 \\
33.784\end{array}$ & $\begin{array}{l}28.000 \\
29.333 \\
30.667 \\
32.000 \\
33.333\end{array}$ & $\begin{array}{l}27.632 \\
28.947 \\
30.263 \\
31.579 \\
32.895\end{array}$ & $\begin{array}{l}27.273 \\
28.571 \\
29.870 \\
31.169 \\
32.468\end{array}$ & $\begin{array}{l}26.923 \\
28.205 \\
29.487 \\
30.769 \\
32.051\end{array}$ & $\begin{array}{l}26.582 \\
27.848 \\
29.114 \\
30.380 \\
31.646\end{array}$ & $\begin{array}{l}26.250 \\
27.500 \\
28.750 \\
30.000 \\
31.250\end{array}$ \\
\hline $\begin{array}{l}26 \\
27 \\
28 \\
29 \\
30\end{array}$ & $\begin{array}{l}36.620 \\
38.028 \\
39.437 \\
40.845 \\
42.254\end{array}$ & $\begin{array}{l}36.111 \\
37.500 \\
38.889 \\
40.278 \\
41.667\end{array}$ & $\begin{array}{l}35.616 \\
36.986 \\
38.356 \\
39.726 \\
41.096\end{array}$ & $\begin{array}{l}35.135 \\
36.486 \\
37.838 \\
39.189 \\
40.541\end{array}$ & $\begin{array}{l}34.667 \\
36.000 \\
37.333 \\
38.667 \\
40.000\end{array}$ & $\begin{array}{l}34.211 \\
35.526 \\
36.842 \\
38.158 \\
39.474\end{array}$ & $\begin{array}{l}33.766 \\
35.065 \\
36.364 \\
37.662 \\
38.961\end{array}$ & $\begin{array}{l}33.333 \\
34.615 \\
35.897 \\
37.179 \\
38.462\end{array}$ & $\begin{array}{l}32.911 \\
34.177 \\
35.443 \\
36.709 \\
37.975\end{array}$ & $\begin{array}{l}32.500 \\
33.750 \\
35.000 \\
36.250 \\
37.500\end{array}$ \\
\hline $\begin{array}{l}31 \\
32 \\
33 \\
34 \\
35\end{array}$ & $\begin{array}{l}43.662 \\
45.070 \\
46.479 \\
47.887 \\
49.296\end{array}$ & $\begin{array}{l}43.056 \\
44.444 \\
45.833 \\
47.222 \\
48.611\end{array}$ & $\begin{array}{l}42.466 \\
43.836 \\
45.205 \\
46.575 \\
47.945\end{array}$ & $\begin{array}{l}41.892 \\
43.243 \\
44.595 \\
45.946 \\
47.297\end{array}$ & $\begin{array}{l}41.333 \\
42.667 \\
44.000 \\
45.333 \\
46.667\end{array}$ & $\begin{array}{l}40.789 \\
42.105 \\
43.421 \\
44.737 \\
46.053\end{array}$ & $\begin{array}{l}40.260 \\
41.558 \\
42.857 \\
44.156 \\
45.455\end{array}$ & $\begin{array}{l}39.744 \\
41.026 \\
42.308 \\
43.590 \\
44.872\end{array}$ & $\begin{array}{l}39.241 \\
40.506 \\
41.772 \\
43.038 \\
44.304\end{array}$ & $\begin{array}{l}38.750 \\
40.000 \\
41.250 \\
42.500 \\
43.750\end{array}$ \\
\hline $\begin{array}{l}36 \\
37 \\
38 \\
39 \\
40\end{array}$ & $\begin{array}{l}50.704 \\
52.113 \\
53.521 \\
54.930 \\
56.338\end{array}$ & $\begin{array}{l}50.000 \\
51.389 \\
52.778 \\
54.167 \\
55.556\end{array}$ & $\begin{array}{l}49.315 \\
50.685 \\
52.055 \\
53.425 \\
54.795\end{array}$ & $\begin{array}{l}48.649 \\
50.000 \\
51.351 \\
52.703 \\
54.054\end{array}$ & $\begin{array}{l}48.000 \\
49.333 \\
50.667 \\
52.000 \\
53.333\end{array}$ & $\begin{array}{l}47.368 \\
48.684 \\
50.000 \\
51.316 \\
52.632\end{array}$ & $\begin{array}{l}46.753 \\
48.052 \\
49.351 \\
50.649 \\
51.948\end{array}$ & $\begin{array}{l}46.154 \\
47.436 \\
48.718 \\
50.000 \\
51.282\end{array}$ & $\begin{array}{l}45.570 \\
46.835 \\
48.101 \\
49.367 \\
50.633\end{array}$ & $\begin{array}{l}45.000 \\
46.250 \\
47.500 \\
48.750 \\
50.000\end{array}$ \\
\hline $\begin{array}{l}47 \\
42 \\
43 \\
44 \\
45\end{array}$ & $\begin{array}{l}57.746 \\
59.155 \\
60.563 \\
61.972 \\
63.380\end{array}$ & $\begin{array}{l}56.944 \\
58.333 \\
59.722 \\
61.111 \\
62.500\end{array}$ & $\begin{array}{l}56.164 \\
57.534 \\
58.904 \\
60.274 \\
61.644\end{array}$ & $\begin{array}{l}55.405 \\
56.757 \\
58.108 \\
59.459 \\
60.811\end{array}$ & $\begin{array}{l}54.667 \\
56.000 \\
57.333 \\
58.667 \\
60.000\end{array}$ & $\begin{array}{l}53.947 \\
55.263 \\
56.579 \\
57.895 \\
59.211\end{array}$ & $\begin{array}{l}53.247 \\
54.545 \\
55.844 \\
57.143 \\
58.442\end{array}$ & $\begin{array}{l}52.564 \\
53.846 \\
55.128 \\
56.410 \\
57.692\end{array}$ & $\begin{array}{l}51.899 \\
53.165 \\
54.430 \\
55.696 \\
56.962\end{array}$ & $\begin{array}{l}51.250 \\
52.500 \\
53.750 \\
55.000 \\
56.250\end{array}$ \\
\hline $\begin{array}{l}46 \\
47 \\
48 \\
49 \\
50\end{array}$ & $\begin{array}{l}64.789 \\
66.197 \\
67.606 \\
69.014 \\
70.423\end{array}$ & $\begin{array}{l}63.889 \\
65.278 \\
66.667 \\
68.056 \\
69.444\end{array}$ & $\begin{array}{l}63.014 \\
64.384 \\
65.753 \\
67.123 \\
68.493\end{array}$ & $\begin{array}{l}62.162 \\
63.514 \\
64.865 \\
66.216 \\
67.568\end{array}$ & $\begin{array}{l}61.333 \\
62.667 \\
64.000 \\
65.333 \\
66.667\end{array}$ & $\begin{array}{l}60.526 \\
61.842 \\
63.158 \\
64.474 \\
65.789\end{array}$ & $\begin{array}{l}59.740 \\
61.039 \\
62.338 \\
63.636 \\
64.935\end{array}$ & $\begin{array}{l}58.974 \\
60.256 \\
61.538 \\
62.821 \\
64.103\end{array}$ & $\begin{array}{l}58.228 \\
59.494 \\
60.759 \\
62.025 \\
63.291\end{array}$ & $\begin{array}{l}57.500 \\
58.750 \\
60.000 \\
61.250 \\
62.500\end{array}$ \\
\hline
\end{tabular}



PERCENTAGE TABLES

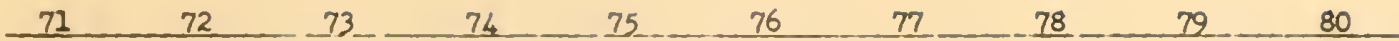

\begin{tabular}{|c|c|c|c|c|c|c|c|c|c|}
\hline $\begin{array}{l}71.831 \\
73.239 \\
74.648 \\
76.056 \\
77.465\end{array}$ & $\begin{array}{l}70.833 \\
72.222 \\
73.611 \\
75.000 \\
76.389\end{array}$ & $\begin{array}{l}69.863 \\
71.233 \\
72.603 \\
73.973 \\
75.342\end{array}$ & $\begin{array}{l}68.919 \\
70.270 \\
71.622 \\
72.973 \\
74.324\end{array}$ & $\begin{array}{l}68.000 \\
69.333 \\
70.667 \\
72.000 \\
73.333\end{array}$ & $\begin{array}{l}67.105 \\
68.421 \\
69.737 \\
71.053 \\
72.368\end{array}$ & $\begin{array}{l}66.234 \\
67.532 \\
68.831 \\
70.130 \\
71.429\end{array}$ & $\begin{array}{l}65.385 \\
66.667 \\
67.949 \\
69.231 \\
70.513\end{array}$ & $\begin{array}{l}64.557 \\
65.823 \\
67.089 \\
68.354 \\
69.620\end{array}$ & $\begin{array}{l}63.750 \\
65.000 \\
66.250 \\
67.500 \\
68.750\end{array}$ \\
\hline $\begin{array}{l}78.873 \\
80.282 \\
81.690 \\
83.099 \\
84.507\end{array}$ & $\begin{array}{l}77.778 \\
79.167 \\
80.556 \\
81.944 \\
83.333\end{array}$ & $\begin{array}{l}76.712 \\
78.082 \\
79.452 \\
80.822 \\
82.192\end{array}$ & $\begin{array}{l}75.676 \\
77.027 \\
78.378 \\
79.730 \\
81.081\end{array}$ & $\begin{array}{l}74.667 \\
76.000 \\
77.333 \\
78.667 \\
80.000\end{array}$ & $\begin{array}{l}73.684 \\
75.000 \\
76.316 \\
77.632 \\
78.947\end{array}$ & $\begin{array}{l}72.727 \\
74.026 \\
75.325 \\
76.623 \\
77.922\end{array}$ & $\begin{array}{l}71.795 \\
73.077 \\
74.359 \\
75.641 \\
76.923\end{array}$ & $\begin{array}{l}70.886 \\
72.152 \\
73.418 \\
74.684 \\
75.949\end{array}$ & $\begin{array}{l}70.000 \\
71.250 \\
72.500 \\
73.750 \\
75.000\end{array}$ \\
\hline $\begin{array}{l}85.915 \\
87.324 \\
88.732 \\
90.141 \\
91.549\end{array}$ & $\begin{array}{l}84.722 \\
86.111 \\
87.500 \\
88.889 \\
90.278\end{array}$ & $\begin{array}{l}83.562 \\
84.932 \\
86.301 \\
87.671 \\
89.041\end{array}$ & $\begin{array}{l}82.432 \\
83.784 \\
85.135 \\
86.486 \\
87.838\end{array}$ & $\begin{array}{l}81.333 \\
82.667 \\
84.000 \\
85.333 \\
86.667\end{array}$ & $\begin{array}{l}80.263 \\
81.579 \\
82.895 \\
84.211 \\
85.526\end{array}$ & $\begin{array}{l}79.221 \\
80.519 \\
81.818 \\
83.117 \\
84.416\end{array}$ & $\begin{array}{l}78.205 \\
79.487 \\
80.769 \\
82.051 \\
83.333\end{array}$ & $\begin{array}{l}77.215 \\
78.481 \\
79.747 \\
81.013 \\
82.278\end{array}$ & $\begin{array}{l}76.25 \\
77.50 \\
78.75 \\
80.00 \\
81.25\end{array}$ \\
\hline $\begin{array}{l}92.958 \\
94.366 \\
95.775 \\
97.183 \\
98.592\end{array}$ & $\begin{array}{l}91.667 \\
93.056 \\
94.444 \\
95.833 \\
97.222\end{array}$ & $\begin{array}{l}90.411 \\
91.781 \\
93.151 \\
94.521 \\
95.890\end{array}$ & $\begin{array}{l}89.189 \\
90.541 \\
91.892 \\
93.243 \\
94.595\end{array}$ & $\begin{array}{l}88.000 \\
89.333 \\
90.667 \\
92.000 \\
93.333\end{array}$ & $\begin{array}{l}86.842 \\
88.158 \\
89.474 \\
90.789 \\
92.105\end{array}$ & $\begin{array}{l}85.714 \\
87.013 \\
88.312 \\
89.610 \\
90.909\end{array}$ & $\begin{array}{l}84.615 \\
85.897 \\
87.179 \\
88.462 \\
89.744\end{array}$ & $\begin{array}{l}83.544 \\
84.810 \\
86.076 \\
87.342 \\
88.608\end{array}$ & $\begin{array}{l}82.50 \\
83.75 \\
85.00 \\
86.25 \\
87.50\end{array}$ \\
\hline $\begin{array}{c}100.000 \\
- \\
- \\
-\end{array}$ & $\begin{array}{c}98.611 \\
100.000 \\
- \\
-\end{array}$ & $\begin{array}{r}97.260 \\
98.630 \\
100.000 \\
-\end{array}$ & $\begin{array}{c}95.946 \\
97.297 \\
98.649 \\
100.000 \\
-\end{array}$ & $\begin{array}{r}94.667 \\
96.000 \\
97.333 \\
98.667 \\
100.000\end{array}$ & $\begin{array}{l}93.421 \\
94.737 \\
96.053 \\
97.368 \\
98.684\end{array}$ & $\begin{array}{l}92.208 \\
93.506 \\
94.805 \\
96.104 \\
97.403\end{array}$ & $\begin{array}{l}91.026 \\
92.308 \\
93.590 \\
94.872 \\
96.154\end{array}$ & $\begin{array}{l}89.873 \\
91.139 \\
92.405 \\
93.671 \\
94.937\end{array}$ & $\begin{array}{l}88.75 \\
90.00 \\
91.25 \\
92.50 \\
93.75\end{array}$ \\
\hline$\overline{-}$ & $\begin{array}{c}-- \\
-- \\
--\end{array}$ & $\begin{array}{c}-- \\
-- \\
--\end{array}$ & - & $\overline{-}$ & $\begin{array}{c}100.000 \\
\ldots \\
- \\
-\end{array}$ & $\begin{array}{c}98.701 \\
100.000 \\
- \\
-\end{array}$ & $\begin{array}{c}97.436 \\
98.718 \\
100.000 \\
\ldots \\
\ldots\end{array}$ & $\begin{array}{r}96.203 \\
97.468 \\
98.734 \\
100.000 \\
\end{array}$ & $\begin{array}{r}95.00 \\
96.25 \\
97.50 \\
98.75 \\
100.00\end{array}$ \\
\hline
\end{tabular}


TERCENTAGE TABLES

\begin{tabular}{|c|c|c|c|c|c|c|c|c|c|c|}
\hline & 81 & 82 & 83 & 84 & 85 & 86 & 87 & 88 & 89 & 90 \\
\hline $\begin{array}{l}1 . \\
2 \\
3 \\
4 \\
5\end{array}$ & $\begin{array}{l}1.235 \\
2.469 \\
3.704 \\
4.938 \\
6.173\end{array}$ & $\begin{array}{l}1.220 \\
2.439 \\
3.659 \\
4.878 \\
6.098\end{array}$ & $\begin{array}{l}1.205 \\
2.410 \\
3.614 \\
4.819 \\
6.024\end{array}$ & $\begin{array}{l}1.190 \\
2.381 \\
3.571 \\
4.762 \\
5.952\end{array}$ & $\begin{array}{l}1.176 \\
2.353 \\
3.529 \\
4.706 \\
5.882\end{array}$ & $\begin{array}{l}1.163 \\
2.326 \\
3.488 \\
4.651 \\
5.814\end{array}$ & $\begin{array}{l}1.149 \\
2.299 \\
3.448 \\
4.598 \\
5.747\end{array}$ & $\begin{array}{l}1.136 \\
2.273 \\
3.409 \\
4.545 \\
5.682\end{array}$ & $\begin{array}{l}1.124 \\
2.247 \\
3.371 \\
4.494 \\
5.618\end{array}$ & $\begin{array}{l}1.111 \\
2.222 \\
3.333 \\
4.444 \\
5.556\end{array}$ \\
\hline $\begin{array}{r}6 \\
7 \\
8 \\
9 \\
10\end{array}$ & $\begin{array}{r}7.407 \\
8.642 \\
9.877 \\
11.111 \\
12.346\end{array}$ & $\begin{array}{r}7.317 \\
8.537 \\
9.756 \\
10.976 \\
12.195\end{array}$ & $\begin{array}{r}7.229 \\
8.434 \\
9.639 \\
10.843 \\
12.048\end{array}$ & $\begin{array}{r}7.143 \\
8.333 \\
9.524 \\
10.714 \\
11.905\end{array}$ & $\begin{array}{r}7.059 \\
8.235 \\
9.412 \\
10.588 \\
11.765\end{array}$ & $\begin{array}{r}6.977 \\
8.140 \\
9.302 \\
10.465 \\
11.628\end{array}$ & $\begin{array}{r}6.897 \\
8.046 \\
9.195 \\
10.345 \\
11.494\end{array}$ & $\begin{array}{r}6.818 \\
7.955 \\
9.091 \\
10.227 \\
11.364\end{array}$ & $\begin{array}{r}6.742 \\
7.865 \\
8.989 \\
10.112 \\
11.236\end{array}$ & $\begin{array}{r}6.667 \\
7.778 \\
8.889 \\
10.000 \\
11.111\end{array}$ \\
\hline $\begin{array}{l}11 \\
12 \\
13 \\
14 \\
15\end{array}$ & $\begin{array}{l}13.580 \\
14.815 \\
16.049 \\
17.284 \\
18.519\end{array}$ & $\begin{array}{l}13.415 \\
14.634 \\
15.854 \\
17.073 \\
18.293\end{array}$ & $\begin{array}{l}13.253 \\
14.458 \\
15.663 \\
16.867 \\
18.072\end{array}$ & $\begin{array}{l}13.095 \\
14.286 \\
15.476 \\
16.667 \\
17.857\end{array}$ & $\begin{array}{l}12.941 \\
14.118 \\
15.294 \\
16.471 \\
17.647\end{array}$ & $\begin{array}{l}12.791 \\
13.953 \\
15.116 \\
16.279 \\
17.442\end{array}$ & $\begin{array}{l}12.644 \\
13.793 \\
14.943 \\
16.092 \\
17.241\end{array}$ & $\begin{array}{l}12.500 \\
13.636 \\
14.773 \\
15.909 \\
17.045\end{array}$ & $\begin{array}{l}12.360 \\
13.483 \\
14.607 \\
15.730 \\
16.854\end{array}$ & $\begin{array}{l}12.222 \\
13.333 \\
14.444 \\
15.556 \\
16.667\end{array}$ \\
\hline $\begin{array}{l}16 \\
17 \\
18 \\
19 \\
20\end{array}$ & $\begin{array}{l}19.753 \\
20.988 \\
22.222 \\
23.457 \\
24.691\end{array}$ & $\begin{array}{l}19.512 \\
20.732 \\
21.951 \\
23.171 \\
24.390\end{array}$ & $\begin{array}{l}19.277 \\
20.482 \\
21.687 \\
22.892 \\
24.096\end{array}$ & $\begin{array}{l}19.048 \\
20.238 \\
21.429 \\
22.619 \\
23.810\end{array}$ & $\begin{array}{l}18.824 \\
20.000 \\
21.176 \\
22.353 \\
23.529\end{array}$ & $\begin{array}{l}18.605 \\
19.767 \\
20.930 \\
22.093 \\
23.256\end{array}$ & $\begin{array}{l}18.391 \\
19.540 \\
20.690 \\
21.839 \\
22.989\end{array}$ & $\begin{array}{l}18.182 \\
19.318 \\
20.455 \\
21.591 \\
22.727\end{array}$ & $\begin{array}{l}17.978 \\
19.101 \\
20.225 \\
21.348 \\
22.472\end{array}$ & $\begin{array}{l}17.778 \\
18.889 \\
20.000 \\
21.111 \\
22.222\end{array}$ \\
\hline $\begin{array}{l}21 \\
22 \\
23 \\
24 \\
25\end{array}$ & $\begin{array}{l}25.926 \\
27.160 \\
28.395 \\
29.630 \\
30.864\end{array}$ & $\begin{array}{l}25.610 \\
26.829 \\
28.049 \\
29.268 \\
30.488\end{array}$ & $\begin{array}{l}25.301 \\
26.506 \\
27.711 \\
28.916 \\
30.120\end{array}$ & $\begin{array}{l}25.000 \\
26.190 \\
27.381 \\
28.571 \\
29.762\end{array}$ & $\begin{array}{l}24.706 \\
25.882 \\
27.059 \\
28.235 \\
29.412\end{array}$ & $\begin{array}{l}24.419 \\
25.581 \\
26.744 \\
27.907 \\
29.070\end{array}$ & $\begin{array}{l}24.138 \\
25.287 \\
26.437 \\
27.586 \\
28.736\end{array}$ & $\begin{array}{l}23.864 \\
25.000 \\
26.136 \\
27.273 \\
28.409\end{array}$ & $\begin{array}{l}23.596 \\
24.719 \\
25.843 \\
26.966 \\
28.090\end{array}$ & $\begin{array}{l}23.333 \\
24.444 \\
25.556 \\
26.667 \\
27.778\end{array}$ \\
\hline $\begin{array}{l}26 \\
27 \\
28 \\
29 \\
30\end{array}$ & $\begin{array}{l}32.099 \\
33.333 \\
34.568 \\
35.802 \\
37.037\end{array}$ & $\begin{array}{l}31.707 \\
32.927 \\
34.146 \\
35.366 \\
36.585\end{array}$ & $\begin{array}{l}31.325 \\
32.530 \\
33.735 \\
34.940 \\
36.145\end{array}$ & $\begin{array}{l}30.952 \\
32.143 \\
33.333 \\
34.524 \\
35.714\end{array}$ & $\begin{array}{l}30.588 \\
31.765 \\
32.941 \\
34.118 \\
35.294\end{array}$ & $\begin{array}{l}30.233 \\
31.395 \\
32.558 \\
33.721 \\
34.884\end{array}$ & $\begin{array}{l}29.885 \\
31.034 \\
32.184 \\
33.333 \\
34.483\end{array}$ & $\begin{array}{l}29.545 \\
30.682 \\
31.818 \\
32.955 \\
34.091\end{array}$ & $\begin{array}{l}29.213 \\
30.337 \\
31.461 \\
32.584 \\
33.708\end{array}$ & $\begin{array}{l}28.889 \\
30.000 \\
31.311 \\
32.222 \\
33.333\end{array}$ \\
\hline $\begin{array}{l}31 \\
32 \\
33 \\
34 \\
35\end{array}$ & $\begin{array}{l}38.272 \\
39.506 \\
40.741 \\
41.975 \\
43.210\end{array}$ & $\begin{array}{l}37.805 \\
39.024 \\
40.244 \\
41.463 \\
42.683\end{array}$ & $\begin{array}{l}37.349 \\
38.554 \\
39.759 \\
40.964 \\
42.169\end{array}$ & $\begin{array}{l}36.905 \\
38.095 \\
39.286 \\
40.476 \\
41.667\end{array}$ & $\begin{array}{l}36.471 \\
37.647 \\
38.824 \\
40.000 \\
41.176\end{array}$ & $\begin{array}{l}36.047 \\
37.209 \\
38.372 \\
39.535 \\
40.698\end{array}$ & $\begin{array}{l}35.632 \\
36.782 \\
37.931 \\
39.080 \\
40.230\end{array}$ & $\begin{array}{l}35.227 \\
36.364 \\
37.500 \\
38.636 \\
39.773\end{array}$ & $\begin{array}{l}34.831 \\
35.955 \\
37.079 \\
38.202 \\
39.326\end{array}$ & $\begin{array}{l}34.444 \\
35.556 \\
36.667 \\
37.778 \\
38.889\end{array}$ \\
\hline $\begin{array}{l}36 \\
37 \\
38 \\
39 \\
40\end{array}$ & $\begin{array}{l}44 \cdot 444 \\
45.679 \\
46.914 \\
48.148 \\
49.383\end{array}$ & $\begin{array}{l}43.902 \\
45.122 \\
46.341 \\
47.561 \\
48.780\end{array}$ & $\begin{array}{l}43.373 \\
44.578 \\
45.783 \\
46.988 \\
48.193\end{array}$ & $\begin{array}{l}42.857 \\
44.048 \\
45.238 \\
46.429 \\
47.619\end{array}$ & $\begin{array}{l}42.353 \\
43.529 \\
44.706 \\
45.882 \\
47.059\end{array}$ & $\begin{array}{l}41.860 \\
43.023 \\
44.186 \\
45.349 \\
46.512\end{array}$ & $\begin{array}{l}41.379 \\
42.529 \\
43.678 \\
44.828 \\
45.977\end{array}$ & $\begin{array}{l}40.909 \\
42.045 \\
43.182 \\
44.318 \\
45.455\end{array}$ & $\begin{array}{l}40.449 \\
41.573 \\
42.697 \\
43.820 \\
44.944\end{array}$ & $\begin{array}{l}40.000 \\
41.111 \\
42.222 \\
43.333 \\
44.444\end{array}$ \\
\hline $\begin{array}{l}41 \\
42 \\
43 \\
44 \\
45\end{array}$ & $\begin{array}{l}50.617 \\
51.852 \\
53.086 \\
54.321 \\
55.556\end{array}$ & $\begin{array}{l}50.000 \\
51.220 \\
52.439 \\
53.659 \\
54.878\end{array}$ & $\begin{array}{l}49.398 \\
50.602 \\
51.807 \\
53.012 \\
54.217\end{array}$ & $\begin{array}{l}48.810 \\
50.000 \\
51.190 \\
52.381 \\
53.571\end{array}$ & $\begin{array}{l}48.235 \\
49.412 \\
50.588 \\
51.765 \\
52.941\end{array}$ & $\begin{array}{l}47.674 \\
48.837 \\
50.000 \\
51.163 \\
52.326\end{array}$ & $\begin{array}{l}47.126 \\
48.276 \\
49.425 \\
50.575 \\
51.724\end{array}$ & $\begin{array}{l}46.591 \\
47.727 \\
48.864 \\
50.000 \\
51.136\end{array}$ & $\begin{array}{l}46.067 \\
47.191 \\
48.315 \\
49.438 \\
50.562\end{array}$ & $\begin{array}{l}45.556 \\
46.667 \\
47.778 \\
48.889 \\
50.000\end{array}$ \\
\hline $\begin{array}{l}46 \\
47 \\
48 \\
49 \\
50\end{array}$ & $\begin{array}{l}56.790 \\
58.025 \\
59.259 \\
60.494 \\
61.728\end{array}$ & $\begin{array}{l}56.098 \\
57.317 \\
58.537 \\
59.756 \\
60.976\end{array}$ & $\begin{array}{l}55.422 \\
56.627 \\
57.831 \\
59.036 \\
60.241\end{array}$ & $\begin{array}{l}54.762 \\
55.952 \\
57.143 \\
58.333 \\
59.524\end{array}$ & $\begin{array}{l}54.118 \\
55.294 \\
56.471 \\
57.647 \\
58.824\end{array}$ & $\begin{array}{l}53.488 \\
54.651 \\
55.811 \\
56.977 \\
58.140\end{array}$ & $\begin{array}{l}52.874 \\
54.023 \\
55.172 \\
56.322 \\
57.471\end{array}$ & $\begin{array}{l}52.273 \\
53.109 \\
54.545 \\
55.682 \\
56.818\end{array}$ & $\begin{array}{l}51.685 \\
52.809 \\
53.933 \\
55.056 \\
56.180\end{array}$ & $\begin{array}{l}51.111 \\
52.222 \\
53.333 \\
54.444 \\
55.556\end{array}$ \\
\hline
\end{tabular}





\begin{tabular}{|c|c|c|c|c|c|c|c|c|c|c|}
\hline & 81 & 82 & 83 & 84 & 85 & 86 & 87 & 88 & 89 & 20 \\
\hline $\begin{array}{l}51 \\
52 \\
53 \\
54 \\
55\end{array}$ & $\begin{array}{l}62.963 \\
64.198 \\
65.432 \\
66.667 \\
67.901\end{array}$ & $\begin{array}{l}62.195 \\
63.415 \\
64.634 \\
65.854 \\
67.073\end{array}$ & $\begin{array}{l}61.446 \\
62.651 \\
63.855 \\
65.060 \\
66.265\end{array}$ & $\begin{array}{l}60.714 \\
61.905 \\
63.095 \\
64.286 \\
65.476\end{array}$ & $\begin{array}{l}60.000 \\
61.176 \\
62.353 \\
63.529 \\
64.706\end{array}$ & $\begin{array}{l}59.302 \\
60.465 \\
61.628 \\
62.791 \\
63.953\end{array}$ & $\begin{array}{l}58.621 \\
59.770 \\
60.920 \\
62.069 \\
63.218\end{array}$ & $\begin{array}{l}57.955 \\
59.091 \\
60.227 \\
61.364 \\
62.500\end{array}$ & $\begin{array}{l}57.303 \\
58.427 \\
59.551 \\
60.674 \\
61.798\end{array}$ & $\begin{array}{l}56.667 \\
57.778 \\
58.889 \\
60.000 \\
61.111\end{array}$ \\
\hline $\begin{array}{l}56 \\
57 \\
58 \\
59 \\
60\end{array}$ & $\begin{array}{l}69.136 \\
70.370 \\
71.605 \\
72.840 \\
74.074\end{array}$ & $\begin{array}{l}68.293 \\
69.512 \\
70.732 \\
71.951 \\
73.171\end{array}$ & $\begin{array}{l}67.470 \\
68.675 \\
69.880 \\
71.084 \\
72.289\end{array}$ & $\begin{array}{l}66.667 \\
67.857 \\
69.048 \\
70.238 \\
71.429\end{array}$ & $\begin{array}{l}65.882 \\
67.059 \\
68.235 \\
69.412 \\
70.588\end{array}$ & $\begin{array}{l}65.116 \\
66.279 \\
67.442 \\
68.605 \\
69.767\end{array}$ & $\begin{array}{l}64.368 \\
65.517 \\
66.667 \\
67.816 \\
68.966\end{array}$ & $\begin{array}{l}63.636 \\
64.773 \\
65.909 \\
67.045 \\
68.182\end{array}$ & $\begin{array}{l}62.921 \\
64.045 \\
65.169 \\
66.292 \\
67.416\end{array}$ & $\begin{array}{l}62.222 \\
63.333 \\
64.444 \\
65.556 \\
66.667\end{array}$ \\
\hline $\begin{array}{l}61 \\
62 \\
63 \\
64 \\
65\end{array}$ & $\begin{array}{l}75.309 \\
76.543 \\
77.778 \\
79.012 \\
80.247\end{array}$ & $\begin{array}{l}74.390 \\
75.610 \\
76.829 \\
78.049 \\
79.268\end{array}$ & $\begin{array}{l}73.494 \\
74.699 \\
75.904 \\
77.108 \\
78.313\end{array}$ & $\begin{array}{l}7 \% .619 \\
73.810 \\
75.000 \\
76.190 \\
77.381\end{array}$ & $\begin{array}{l}71.765 \\
72.941 \\
74.118 \\
75.294 \\
76.471\end{array}$ & $\begin{array}{l}70.930 \\
72.093 \\
73.256 \\
74.479 \\
75.581\end{array}$ & $\begin{array}{l}70.115 \\
71.264 \\
72.414 \\
73.563 \\
74.713\end{array}$ & $\begin{array}{l}69.318 \\
70.455 \\
71.591 \\
72.727 \\
73.864\end{array}$ & $\begin{array}{l}68.539 \\
69.663 \\
70.787 \\
71.910 \\
73.034\end{array}$ & $\begin{array}{l}67.778 \\
68.889 \\
70.000 \\
71.111 \\
72.222\end{array}$ \\
\hline $\begin{array}{l}66 \\
67 \\
68 \\
69 \\
70\end{array}$ & $\begin{array}{l}81.481 \\
82.716 \\
83.951 \\
85.185 \\
86.420\end{array}$ & $\begin{array}{l}80.488 \\
81.707 \\
82.927 \\
84.146 \\
85.366\end{array}$ & $\begin{array}{l}79.518 \\
80.723 \\
81.928 \\
83.133 \\
84.337\end{array}$ & $\begin{array}{l}78.571 \\
79.762 \\
80.952 \\
82.11 .3 \\
83.333\end{array}$ & $\begin{array}{l}77.647 \\
78.824 \\
80.000 \\
81.176 \\
82.353\end{array}$ & $\begin{array}{l}76.744 \\
77.907 \\
79.070 \\
80.233 \\
81.395\end{array}$ & $\begin{array}{l}75.862 \\
77.011 \\
78.161 \\
79.310 \\
80.460\end{array}$ & $\begin{array}{l}75.000 \\
76.136 \\
77.273 \\
78.409 \\
79.545\end{array}$ & $\begin{array}{l}74.157 \\
75.281 \\
76.404 \\
77.528 \\
78.652\end{array}$ & $\begin{array}{l}73.333 \\
74.444 \\
75.556 \\
76.667 \\
77.778\end{array}$ \\
\hline $\begin{array}{l}71 \\
72 \\
73 \\
74 \\
75\end{array}$ & $\begin{array}{l}87.654 \\
88.889 \\
90.123 \\
91.358 \\
92.593\end{array}$ & $\begin{array}{l}86.585 \\
87.805 \\
89.024 \\
90.244 \\
91.463\end{array}$ & $\begin{array}{l}85.542 \\
86.747 \\
87.952 \\
89.157 \\
90.361\end{array}$ & $\begin{array}{l}84.524 \\
85.714 \\
86.905 \\
88.095 \\
89.286\end{array}$ & $\begin{array}{l}83.529 \\
84.706 \\
85.882 \\
87.059 \\
88.235\end{array}$ & $\begin{array}{l}82.558 \\
83.721 \\
84.884 \\
86.047 \\
87.209\end{array}$ & $\begin{array}{l}81.609 \\
82.759 \\
83.908 \\
85.057 \\
86.207\end{array}$ & $\begin{array}{l}80.682 \\
81.818 \\
82.955 \\
84.091 \\
85.227\end{array}$ & $\begin{array}{l}79.775 \\
80.899 \\
82.022 \\
83.146 \\
84.270\end{array}$ & $\begin{array}{l}78.889 \\
80.000 \\
81.111 \\
82.222 \\
83.333\end{array}$ \\
\hline $\begin{array}{l}76 \\
77 \\
78 \\
79 \\
80\end{array}$ & $\begin{array}{l}93.827 \\
95.062 \\
96.296 \\
97.531 \\
98.765\end{array}$ & $\begin{array}{l}92.683 \\
93.902 \\
95.122 \\
96.341 \\
97.561\end{array}$ & $\begin{array}{l}91.566 \\
92.771 \\
93.976 \\
95.181 \\
96.386\end{array}$ & $\begin{array}{l}90.476 \\
91.667 \\
92.857 \\
94.048 \\
95.238\end{array}$ & $\begin{array}{l}89.412 \\
90.588 \\
91.765 \\
92.941 \\
94.118\end{array}$ & $\begin{array}{l}88.372 \\
89.535 \\
90.698 \\
91.860 \\
93.023\end{array}$ & $\begin{array}{l}87.356 \\
88.506 \\
89.655 \\
90.805 \\
91.954\end{array}$ & $\begin{array}{l}86.364 \\
87.500 \\
88.636 \\
89.773 \\
90.909\end{array}$ & $\begin{array}{l}85.393 \\
86.517 \\
87.640 \\
88.764 \\
89.888\end{array}$ & $\begin{array}{l}84.444 \\
85.556 \\
86.667 \\
87.778 \\
88.889\end{array}$ \\
\hline $\begin{array}{l}81 \\
82 \\
83 \\
84 \\
85\end{array}$ & $\begin{array}{c}100.000 \\
- \\
- \\
-\end{array}$ & $\begin{array}{c}98.780 \\
100.000 \\
- \\
- \\
-\end{array}$ & $\begin{array}{r}97.590 \\
98.795 \\
100.000 \\
- \\
-\end{array}$ & $\begin{array}{r}96.429 \\
97.619 \\
98.810 \\
100.000 \\
\end{array}$ & $\begin{array}{r}95.294 \\
96.471 \\
97.647 \\
98.824 \\
100.000\end{array}$ & $\begin{array}{l}94.186 \\
95.349 \\
96.512 \\
97.674 \\
98.837\end{array}$ & $\begin{array}{l}93.103 \\
94.253 \\
95.402 \\
96.552 \\
97.701\end{array}$ & $\begin{array}{l}92.045 \\
93.182 \\
94.318 \\
95.455 \\
96.591\end{array}$ & $\begin{array}{l}91.011 \\
92.135 \\
93.258 \\
94.382 \\
95.506\end{array}$ & $\begin{array}{l}90.000 \\
91.111 \\
92.222 \\
93.333 \\
94.444\end{array}$ \\
\hline $\begin{array}{l}86 \\
87 \\
88 \\
89 \\
90\end{array}$ & 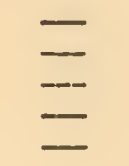 & $\overline{--}$ & $\begin{array}{l}-- \\
-- \\
--\end{array}$ & $\begin{array}{l}-- \\
-- \\
--- \\
--\end{array}$ & $\begin{array}{l}-- \\
-- \\
-- \\
--\end{array}$ & $\begin{array}{c}100.000 \\
- \\
- \\
- \\
-\end{array}$ & $\begin{array}{c}98.851 \\
100.000 \\
\ldots \\
- \\
-\end{array}$ & $\begin{array}{c}97.727 \\
98.864 \\
100.000 \\
\ldots- \\
-\end{array}$ & $\begin{array}{r}96.629 \\
97.753 \\
98.876 \\
100.000 \\
\ldots\end{array}$ & $\begin{array}{r}95.556 \\
96.667 \\
97.778 \\
98.889 \\
100.000\end{array}$ \\
\hline
\end{tabular}





\begin{tabular}{|c|c|c|c|c|c|c|c|c|c|c|}
\hline & 91 & 92 & 93 & 94 & 95 & 96 & 27 & 98 & 92 & 100 \\
\hline $\begin{array}{l}1 \\
2 \\
3 \\
4 \\
5\end{array}$ & $\begin{array}{l}1.099 \\
2.198 \\
3.297 \\
4.396 \\
5.495\end{array}$ & $\begin{array}{l}1.087 \\
2.174 \\
3.261 \\
4.348 \\
5.435\end{array}$ & $\begin{array}{l}1.075 \\
2.151 \\
3.226 \\
4.301 \\
5.376\end{array}$ & $\begin{array}{l}1.064 \\
2.128 \\
3.191 \\
4.255 \\
5.319\end{array}$ & $\begin{array}{l}1.053 \\
2.105 \\
3.158 \\
4.211 \\
5.263\end{array}$ & $\begin{array}{l}1.042 \\
2.083 \\
3.125 \\
4.167 \\
5.208\end{array}$ & $\begin{array}{l}1.031 \\
2.062 \\
3.093 \\
4.124 \\
5.155\end{array}$ & $\begin{array}{l}1.020 \\
2.041 \\
3.061 \\
4.082 \\
5.102\end{array}$ & $\begin{array}{l}1.010 \\
2.020 \\
3.030 \\
4.040 \\
5.051\end{array}$ & $\begin{array}{l}1.000 \\
2.000 \\
3.000 \\
4.000 \\
5.000\end{array}$ \\
\hline $\begin{array}{r}6 \\
7 \\
8 \\
9 \\
10\end{array}$ & $\begin{array}{r}6.593 \\
7.692 \\
8.791 \\
9.890 \\
10.989\end{array}$ & $\begin{array}{r}6.522 \\
7.609 \\
8.696 \\
9.783 \\
10.870\end{array}$ & $\begin{array}{r}6.452 \\
7.527 \\
8.602 \\
9.677 \\
10.753\end{array}$ & $\begin{array}{r}6.383 \\
7.447 \\
8.511 \\
9.574 \\
10.638\end{array}$ & $\begin{array}{r}6.316 \\
7.368 \\
8.421 \\
9.474 \\
10.526\end{array}$ & $\begin{array}{r}6.250 \\
7.292 \\
8.333 \\
9.375 \\
10.417\end{array}$ & $\begin{array}{r}6.186 \\
7.216 \\
8.247 \\
9.278 \\
10.309\end{array}$ & $\begin{array}{r}6.122 \\
7.143 \\
8.163 \\
9.184 \\
10.204\end{array}$ & $\begin{array}{r}6.061 \\
7.071 \\
8.081 \\
9.091 \\
10.101\end{array}$ & $\begin{array}{r}6.000 \\
7.000 \\
8.000 \\
9.000 \\
10.000\end{array}$ \\
\hline $\begin{array}{l}12 \\
13 \\
14 \\
15\end{array}$ & $\begin{array}{l}12.088 \\
13.187 \\
14.286 \\
15.385 \\
16.484\end{array}$ & $\begin{array}{l}11.957 \\
13.043 \\
14.130 \\
15.217 \\
16.304\end{array}$ & $\begin{array}{l}11.828 \\
12.903 \\
13.978 \\
15.054 \\
16.129\end{array}$ & $\begin{array}{l}11.702 \\
12.766 \\
13.830 \\
14.894 \\
15.957\end{array}$ & $\begin{array}{l}11.579 \\
12.632 \\
13.684 \\
14.737 \\
15.789\end{array}$ & $\begin{array}{l}11.458 \\
12.500 \\
13.542 \\
14.583 \\
15.625\end{array}$ & $\begin{array}{l}11.340 \\
12.371 \\
13.402 \\
14.433 \\
15.464\end{array}$ & $\begin{array}{l}11.224 \\
12.245 \\
13.265 \\
14.286 \\
15.306\end{array}$ & $\begin{array}{l}11.111 \\
12.121 \\
13.131 \\
14.141 \\
15.152\end{array}$ & $\begin{array}{l}11.000 \\
12.000 \\
13.000 \\
14.000 \\
15.000\end{array}$ \\
\hline 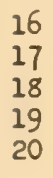 & $\begin{array}{l}17.582 \\
18.681 \\
19.780 \\
20.879 \\
21.978\end{array}$ & $\begin{array}{l}17.391 \\
18.478 \\
19.565 \\
20.652 \\
21.739\end{array}$ & $\begin{array}{l}17.204 \\
18.280 \\
19.355 \\
20.430 \\
22.505\end{array}$ & $\begin{array}{l}17.021 \\
18.085 \\
19.149 \\
20.213 \\
21.277\end{array}$ & $\begin{array}{l}16.842 \\
17.895 \\
18.947 \\
20.000 \\
21.053\end{array}$ & $\begin{array}{l}16.667 \\
17.708 \\
18.750 \\
19.792 \\
20.833\end{array}$ & $\begin{array}{l}16.495 \\
17.526 \\
18.557 \\
19.588 \\
20.619\end{array}$ & $\begin{array}{l}16.327 \\
17.347 \\
18.367 \\
19.388 \\
20.408\end{array}$ & $\begin{array}{l}16.162 \\
17.172 \\
18.182 \\
19.192 \\
20.202\end{array}$ & $\begin{array}{l}16.000 \\
17.000 \\
18.000 \\
19.000 \\
20.000\end{array}$ \\
\hline $\begin{array}{l}22 \\
23 \\
24 \\
25\end{array}$ & $\begin{array}{l}23.077 \\
24.176 \\
25.275 \\
26.374 \\
27.473\end{array}$ & $\begin{array}{l}22.826 \\
23.913 \\
25.000 \\
26.087 \\
27.174\end{array}$ & $\begin{array}{l}22.581 \\
23.656 \\
24.731 \\
25.806 \\
26.882\end{array}$ & $\begin{array}{l}22.340 \\
23.404 \\
24.468 \\
25.532 \\
26.596\end{array}$ & $\begin{array}{l}22.105 \\
23.158 \\
24.211 \\
25.263 \\
26.316\end{array}$ & $\begin{array}{l}21.875 \\
22.917 \\
23.958 \\
25.000 \\
26.042\end{array}$ & $\begin{array}{l}21.649 \\
22.680 \\
23.711 \\
24.742 \\
25.773\end{array}$ & $\begin{array}{l}21.429 \\
22.449 \\
23.469 \\
24.490 \\
25.510\end{array}$ & $\begin{array}{l}21.212 \\
22.222 \\
23.232 \\
24.242 \\
25.253\end{array}$ & $\begin{array}{l}21.000 \\
22.000 \\
23.000 \\
24.000 \\
25.000\end{array}$ \\
\hline $\begin{array}{l}26 \\
27 \\
28 \\
29 \\
30\end{array}$ & $\begin{array}{l}28.571 \\
29.670 \\
30.769 \\
31.868 \\
32.967\end{array}$ & $\begin{array}{l}28.261 \\
29.348 \\
30.435 \\
31.522 \\
32.609\end{array}$ & $\begin{array}{l}27.957 \\
29.032 \\
30.108 \\
31.183 \\
32.258\end{array}$ & $\begin{array}{l}27.660 \\
28.723 \\
29.787 \\
30.851 \\
31.915\end{array}$ & $\begin{array}{l}27.368 \\
28.421 \\
29.474 \\
30.526 \\
31.579\end{array}$ & $\begin{array}{l}27.083 \\
28.125 \\
29.167 \\
30.208 \\
31.250\end{array}$ & $\begin{array}{l}26.804 \\
27.835 \\
28.866 \\
29.897 \\
30.928\end{array}$ & $\begin{array}{l}26.531 \\
27.551 \\
28.571 \\
29.592 \\
30.612\end{array}$ & $\begin{array}{l}26.263 \\
27.273 \\
28.283 \\
29.293 \\
30.303\end{array}$ & $\begin{array}{l}26.000 \\
27.000 \\
28.000 \\
29.000 \\
30.000\end{array}$ \\
\hline $\begin{array}{l}31 \\
32 \\
33 \\
34 \\
35\end{array}$ & $\begin{array}{l}34.066 \\
35.164 \\
36.264 \\
37.363 \\
38.462\end{array}$ & $\begin{array}{l}33.696 \\
34.783 \\
35.870 \\
36.957 \\
38.043\end{array}$ & $\begin{array}{l}33.333 \\
34.509 \\
35.484 \\
36.559 \\
37.634\end{array}$ & $\begin{array}{l}32.979 \\
34.043 \\
35.106 \\
36.170 \\
37.234\end{array}$ & $\begin{array}{l}32.632 \\
33.684 \\
34.737 \\
35.789 \\
36.842\end{array}$ & $\begin{array}{l}32.292 \\
33.333 \\
34.375 \\
35.417 \\
36.458\end{array}$ & $\begin{array}{l}31.959 \\
32.990 \\
34.021 \\
35.052 \\
36.082\end{array}$ & $\begin{array}{l}31.633 \\
32.653 \\
33.673 \\
34.694 \\
35.714\end{array}$ & $\begin{array}{l}31.313 \\
32.323 \\
33.333 \\
34.343 \\
35.354\end{array}$ & $\begin{array}{l}31.000 \\
32.000 \\
33.000 \\
34.000 \\
35.000\end{array}$ \\
\hline $\begin{array}{l}36 \\
37 \\
38 \\
39\end{array}$ & $\begin{array}{l}39.560 \\
40.659 \\
41.758 \\
42.857 \\
43.956\end{array}$ & $\begin{array}{l}39.130 \\
40.217 \\
41.304 \\
42.391 \\
43.478\end{array}$ & $\begin{array}{l}38.710 \\
33.785 \\
40.860 \\
41.935 \\
43.011\end{array}$ & $\begin{array}{l}38.298 \\
39.362 \\
40.426 \\
41.489 \\
42.553\end{array}$ & $\begin{array}{l}37.895 \\
38.947 \\
40.000 \\
41.053 \\
42.105\end{array}$ & $\begin{array}{l}37.500 \\
38.542 \\
39.583 \\
40.625 \\
41.667\end{array}$ & $\begin{array}{l}37.113 \\
38.144 \\
39.175 \\
40.206 \\
41.237\end{array}$ & $\begin{array}{l}36.735 \\
37.755 \\
38.776 \\
39.796 \\
40.816\end{array}$ & $\begin{array}{l}36.364 \\
37.374 \\
38.384 \\
39.394 \\
40.404\end{array}$ & $\begin{array}{l}36.000 \\
37.000 \\
38.000 \\
39.000 \\
40.000\end{array}$ \\
\hline $\begin{array}{l}41 \\
42 \\
43 \\
44 \\
45\end{array}$ & $\begin{array}{l}45.055 \\
46.154 \\
47.253 \\
48.352 \\
49.451\end{array}$ & $\begin{array}{l}44.565 \\
45.652 \\
46.739 \\
47.826 \\
48.913\end{array}$ & $\begin{array}{l}44.086 \\
45.161 \\
46.237 \\
47.312 \\
48.387\end{array}$ & $\begin{array}{l}43.617 \\
44.681 \\
45.745 \\
46.809 \\
47.872\end{array}$ & $\begin{array}{l}43.158 \\
44.211 \\
45.263 \\
46.316 \\
47.368\end{array}$ & $\begin{array}{l}42.708 \\
43.750 \\
44.792 \\
45.833 \\
46.875\end{array}$ & $\begin{array}{l}42.268 \\
43.299 \\
44.330 \\
45.361 \\
46.392\end{array}$ & $\begin{array}{l}41.837 \\
42.857 \\
43.878 \\
44.898 \\
45.918\end{array}$ & $\begin{array}{l}41.414 \\
42.424 \\
43.434 \\
44.414 \\
45.455\end{array}$ & $\begin{array}{l}41.000 \\
42.000 \\
43.000 \\
4.000 \\
+5.000\end{array}$ \\
\hline $\begin{array}{l}46 \\
47 \\
48 \\
49\end{array}$ & $\begin{array}{l}50.549 \\
51.648 \\
52.747 \\
53.846 \\
54.945\end{array}$ & $\begin{array}{l}50.000 \\
51.087 \\
52.174 \\
53.261 \\
54.348\end{array}$ & $\begin{array}{l}49.462 \\
50.538 \\
51.613 \\
52.688 \\
53.763\end{array}$ & $\begin{array}{l}48.936 \\
50.000 \\
51.064 \\
52.128 \\
53.191\end{array}$ & $\begin{array}{l}48.421 \\
49.474 \\
50.526 \\
51.579 \\
52.632\end{array}$ & $\begin{array}{l}47.917 \\
48.958 \\
50.000 \\
51.042 \\
52.083\end{array}$ & $\begin{array}{l}47.423 \\
48.454 \\
49.485 \\
50.515 \\
51.546\end{array}$ & $\begin{array}{l}46.939 \\
47.959 \\
48.980 \\
50.000 \\
51.020\end{array}$ & $\begin{array}{l}46.465 \\
47.475 \\
48.485 \\
49.495 \\
50.505\end{array}$ & $\begin{array}{l}46.000 \\
47.000 \\
48.000 \\
49.000 \\
50.000\end{array}$ \\
\hline
\end{tabular}





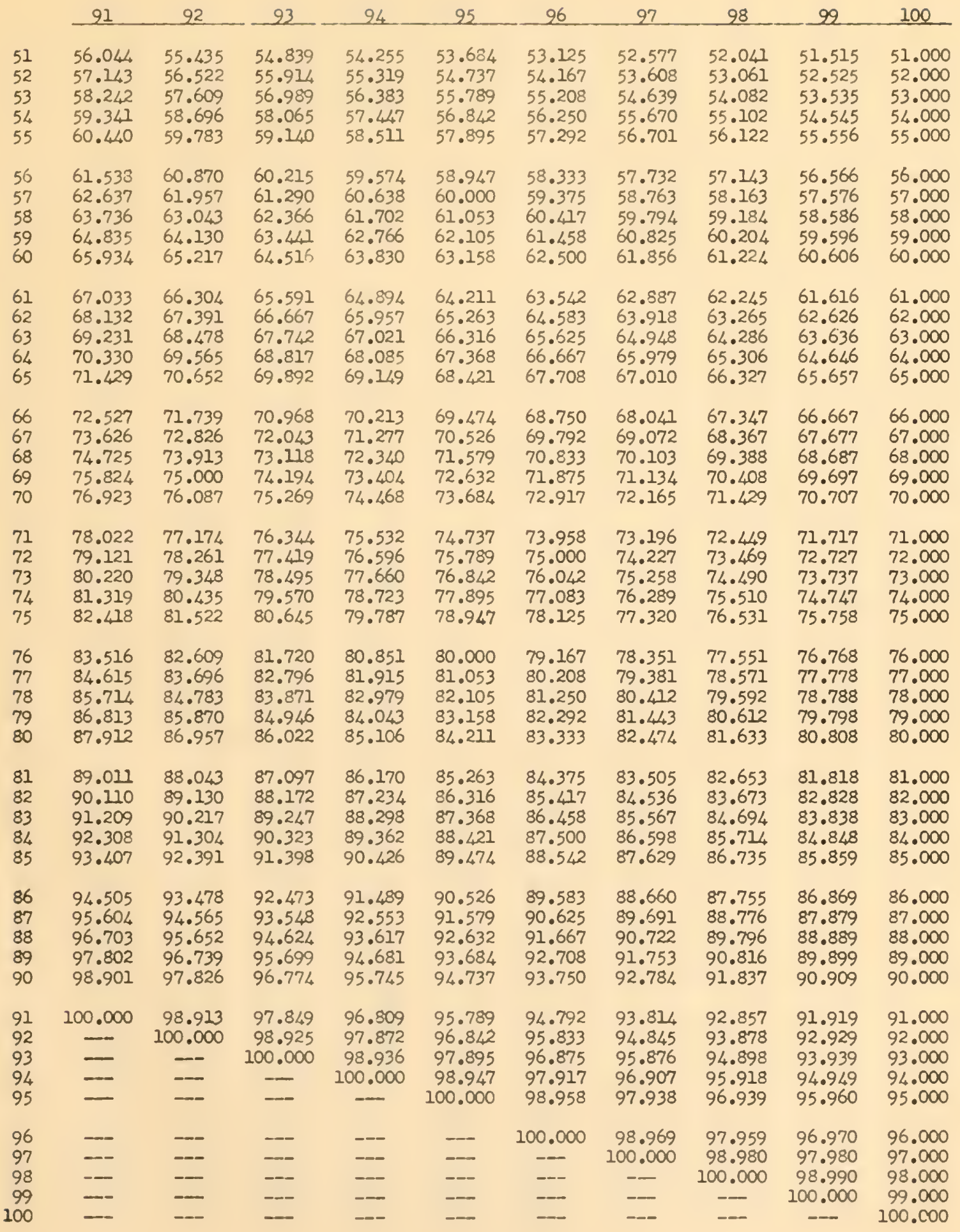





\begin{tabular}{|c|c|c|c|c|c|c|c|c|c|}
\hline 101 & 102 & 103 & 104 & 105 & 106 & 107 & 108 & 109 & 110 \\
\hline $\begin{array}{l}0.990 \\
1.980 \\
2.970 \\
3.960 \\
4.950\end{array}$ & $\begin{array}{l}0.980 \\
1.961 \\
2.941 \\
3.922 \\
4.902\end{array}$ & $\begin{array}{l}0.971 \\
1.942 \\
2.913 \\
3.883 \\
4.854\end{array}$ & $\begin{array}{l}0.962 \\
1.923 \\
2.885 \\
3.846 \\
4.808\end{array}$ & $\begin{array}{l}0.952 \\
1.905 \\
2.857 \\
3.810 \\
4.762\end{array}$ & $\begin{array}{l}0.943 \\
1.887 \\
2.830 \\
3.774 \\
4.717\end{array}$ & $\begin{array}{l}0.935 \\
1.869 \\
2.804 \\
3.738 \\
4.673\end{array}$ & $\begin{array}{l}0.926 \\
1.852 \\
2.778 \\
3.704 \\
4.630\end{array}$ & $\begin{array}{l}0.917 \\
1.835 \\
2.752 \\
3.670 \\
4.587\end{array}$ & $\begin{array}{l}0.909 \\
1.818 \\
2.727 \\
3.636 \\
4.545\end{array}$ \\
\hline $\begin{array}{l}5.941 \\
6.931 \\
7.921 \\
8.911 \\
9.901\end{array}$ & $\begin{array}{l}5.882 \\
6.863 \\
7.843 \\
8.824 \\
9.804\end{array}$ & $\begin{array}{l}5.825 \\
6.796 \\
7.767 \\
8.738 \\
9.709\end{array}$ & $\begin{array}{l}5.769 \\
6.731 \\
7.692 \\
8.654 \\
9.615\end{array}$ & $\begin{array}{l}5.714 \\
6.667 \\
7.619 \\
8.571 \\
9.524\end{array}$ & $\begin{array}{l}5.660 \\
6.604 \\
7.547 \\
8.491 \\
9.434\end{array}$ & $\begin{array}{l}5.607 \\
6.542 \\
7.477 \\
8.411 \\
9.346\end{array}$ & $\begin{array}{l}5.556 \\
6.481 \\
7.407 \\
8.333 \\
9.259\end{array}$ & $\begin{array}{l}5.505 \\
6.422 \\
7.339 \\
8.257 \\
9.174\end{array}$ & $\begin{array}{l}5.455 \\
6.364 \\
7.273 \\
8.182 \\
9.091\end{array}$ \\
\hline $\begin{array}{l}10.891 \\
11.881 \\
12.871 \\
13.861 \\
14.851\end{array}$ & $\begin{array}{l}10.784 \\
11.765 \\
12.745 \\
13.725 \\
14.706\end{array}$ & $\begin{array}{l}10.680 \\
11.650 \\
12.621 \\
13.592 \\
14.563\end{array}$ & $\begin{array}{l}10.577 \\
11.538 \\
12.500 \\
13.462 \\
14.423\end{array}$ & $\begin{array}{l}10.476 \\
11.429 \\
12.381 \\
13.333 \\
14.286\end{array}$ & $\begin{array}{l}10.377 \\
11.321 \\
12.264 \\
13.208 \\
14.151\end{array}$ & $\begin{array}{l}0.280 \\
1.215 \\
2.150 \\
3.084 \\
4.019\end{array}$ & $\begin{array}{l}10.185 \\
11.111 \\
12.037 \\
12.963 \\
13.889\end{array}$ & $\begin{array}{l}10.092 \\
11.009 \\
11.927 \\
12.844 \\
13.761\end{array}$ & $\begin{array}{l}10.000 \\
10.909 \\
11.818 \\
12.727 \\
13.636\end{array}$ \\
\hline $\begin{array}{l}15.842 \\
16.832 \\
17.822 \\
18.812 \\
19.802\end{array}$ & $\begin{array}{l}15.686 \\
16.667 \\
17.647 \\
18.627 \\
19.608\end{array}$ & $\begin{array}{l}15.534 \\
16.505 \\
17.476 \\
18.447 \\
19.417\end{array}$ & $\begin{array}{l}15.385 \\
16.346 \\
17.308 \\
18.269 \\
19.231\end{array}$ & $\begin{array}{l}15 . \angle 3 \circ \\
16.190 \\
17.143 \\
18.095 \\
19.048\end{array}$ & $\begin{array}{l}15.094 \\
16.038 \\
16.981 \\
17.925 \\
18.868\end{array}$ & $\begin{array}{l}14.953 \\
15.888 \\
16.822 \\
17.757 \\
18.692\end{array}$ & $\begin{array}{l}14.815 \\
15.741 \\
16.667 \\
1 . .593 \\
18.519\end{array}$ & $\begin{array}{l}14.679 \\
15.596 \\
16.514 \\
17.431 \\
18.349\end{array}$ & $\begin{array}{l}14.545 \\
15.455 \\
16.364 \\
17.273 \\
18.182\end{array}$ \\
\hline $\begin{array}{l}20.792 \\
22.782 \\
22.772 \\
23.762 \\
24.752\end{array}$ & $\begin{array}{l}20.588 \\
21.569 \\
22.549 \\
23.529 \\
24.510\end{array}$ & $\begin{array}{l}20.388 \\
21.359 \\
22.330 \\
23.301 \\
24.272\end{array}$ & & $\begin{array}{l}20.000 \\
20.952 \\
21.305 \\
22.857 \\
23.810\end{array}$ & $\begin{array}{l}21.698 \\
22.642 \\
23.585\end{array}$ & $\begin{array}{l}19.526 \\
20.561 \\
21.495 \\
22.430 \\
23.364\end{array}$ & $\begin{array}{l}19.414 \\
20.370 \\
21.296 \\
22.222 \\
23.148\end{array}$ & & $\begin{array}{l}19.991 \\
20.000 \\
20.909 \\
21.818 \\
22.727\end{array}$ \\
\hline $\begin{array}{l}25.743 \\
26.733 \\
27.723 \\
28.713 \\
29.703\end{array}$ & $\begin{array}{l}25.490 \\
26.471 \\
27.451 \\
28.431 \\
29.412\end{array}$ & $\begin{array}{l}25.243 \\
26.214 \\
27.184 \\
28.155 \\
29.126\end{array}$ & $\begin{array}{l}25.000 \\
25.962 \\
26.923 \\
27.885 \\
28.846\end{array}$ & $\begin{array}{l}25.714 \\
26.667 \\
27.619 \\
28.571\end{array}$ & $\begin{array}{l}24.528 \\
25.472 \\
26.415 \\
27.358 \\
28.302\end{array}$ & & & $\begin{array}{l}23.853 \\
24.771 \\
25.688 \\
26.606 \\
27.523\end{array}$ & $\begin{array}{l}23.636 \\
24.545 \\
25.455 \\
26.364 \\
27.273\end{array}$ \\
\hline $\begin{array}{l}31.683 \\
32.673 \\
33.663 \\
34.653\end{array}$ & $\begin{array}{l}30.392 \\
31.373 \\
32.353 \\
33.333 \\
34.314\end{array}$ & $\begin{array}{l}30.097 \\
31.068 \\
32.039 \\
33.010 \\
33.981\end{array}$ & & $\begin{array}{l}29.524 \\
30.476 \\
31.429 \\
32.381 \\
33.333\end{array}$ & & & $\begin{array}{l}29.630 \\
30.556 \\
31.481 \\
32.407\end{array}$ & $\begin{array}{l}28.440 \\
29.358 \\
30.275 \\
31.193 \\
32.110\end{array}$ & $\begin{array}{l}28.182 \\
29.091 \\
30.000 \\
30.909 \\
31.518\end{array}$ \\
\hline $\begin{array}{l}35.644 \\
36.634 \\
37.624 \\
38.614 \\
39.604\end{array}$ & $\begin{array}{l}35.294 \\
36.275 \\
37.255 \\
38.235 \\
39.216\end{array}$ & $\begin{array}{l}34.951 \\
35.922 \\
36.893 \\
37.864 \\
38.835\end{array}$ & $\begin{array}{l}34.615 \\
35.577 \\
36.538 \\
37.500 \\
38.462\end{array}$ & $\begin{array}{l}34.286 \\
35.238 \\
36.190 \\
37.143 \\
38.095\end{array}$ & $\begin{array}{l}33.962 \\
34.906 \\
35.849 \\
36.792 \\
37.736\end{array}$ & $\begin{array}{l}33.645 \\
34.579 \\
35.514 \\
36.449 \\
37.383\end{array}$ & & $\begin{array}{l}33.028 \\
33.945 \\
34.862 \\
35.780 \\
36.697\end{array}$ & $\begin{array}{l}32.727 \\
33.636 \\
34.545 \\
35.455 \\
36.364\end{array}$ \\
\hline $\begin{array}{l}40.594 \\
41.584 \\
42.574 \\
43.564 \\
44.554\end{array}$ & $\begin{array}{l}40.196 \\
41.176 \\
42.157 \\
43.137 \\
44.118\end{array}$ & $\begin{array}{l}39.806 \\
40.777 \\
41.748 \\
42.718 \\
43.689\end{array}$ & $\begin{array}{l}39.423 \\
40.385 \\
41.346 \\
42.308\end{array}$ & $\begin{array}{l}39.048 \\
40.000 \\
40.952 \\
41.905 \\
42.857\end{array}$ & $\begin{array}{l}38.679 \\
39.623 \\
40.566 \\
41.509 \\
42.453\end{array}$ & 42.056 & & $\begin{array}{l}37.615 \\
38.532 \\
39.450 \\
40.367 \\
41.284\end{array}$ & $\begin{array}{l}37.273 \\
38.182 \\
39.091 \\
40.000 \\
40.909\end{array}$ \\
\hline $\begin{array}{l}45.545 \\
46.535 \\
47.525 \\
48.515 \\
49.505\end{array}$ & $\begin{array}{l}45.098 \\
46.078 \\
47.059 \\
48.039 \\
49.020\end{array}$ & $\begin{array}{l}44.660 \\
45.631 \\
46.602 \\
47.573 \\
48.544\end{array}$ & $\begin{array}{l}44.231 \\
45.192 \\
46.154 \\
47.115 \\
48.077\end{array}$ & $\begin{array}{l}43.810 \\
44.762 \\
45.714 \\
46.667 \\
47.619\end{array}$ & $\begin{array}{l}45.283 \\
46.226 \\
47.170\end{array}$ & $\begin{array}{l}43.925 \\
44.860 \\
45.794 \\
46.729\end{array}$ & $\begin{array}{l}42.593 \\
43.519 \\
44.441 \\
45.370 \\
46.296\end{array}$ & $\begin{array}{l}42.202 \\
43.119 \\
44.037 \\
44.954 \\
45.872\end{array}$ & $\begin{array}{l}41.818 \\
42.727 \\
43.636 \\
44.545 \\
45.455\end{array}$ \\
\hline
\end{tabular}



PERCENTACE TABLES

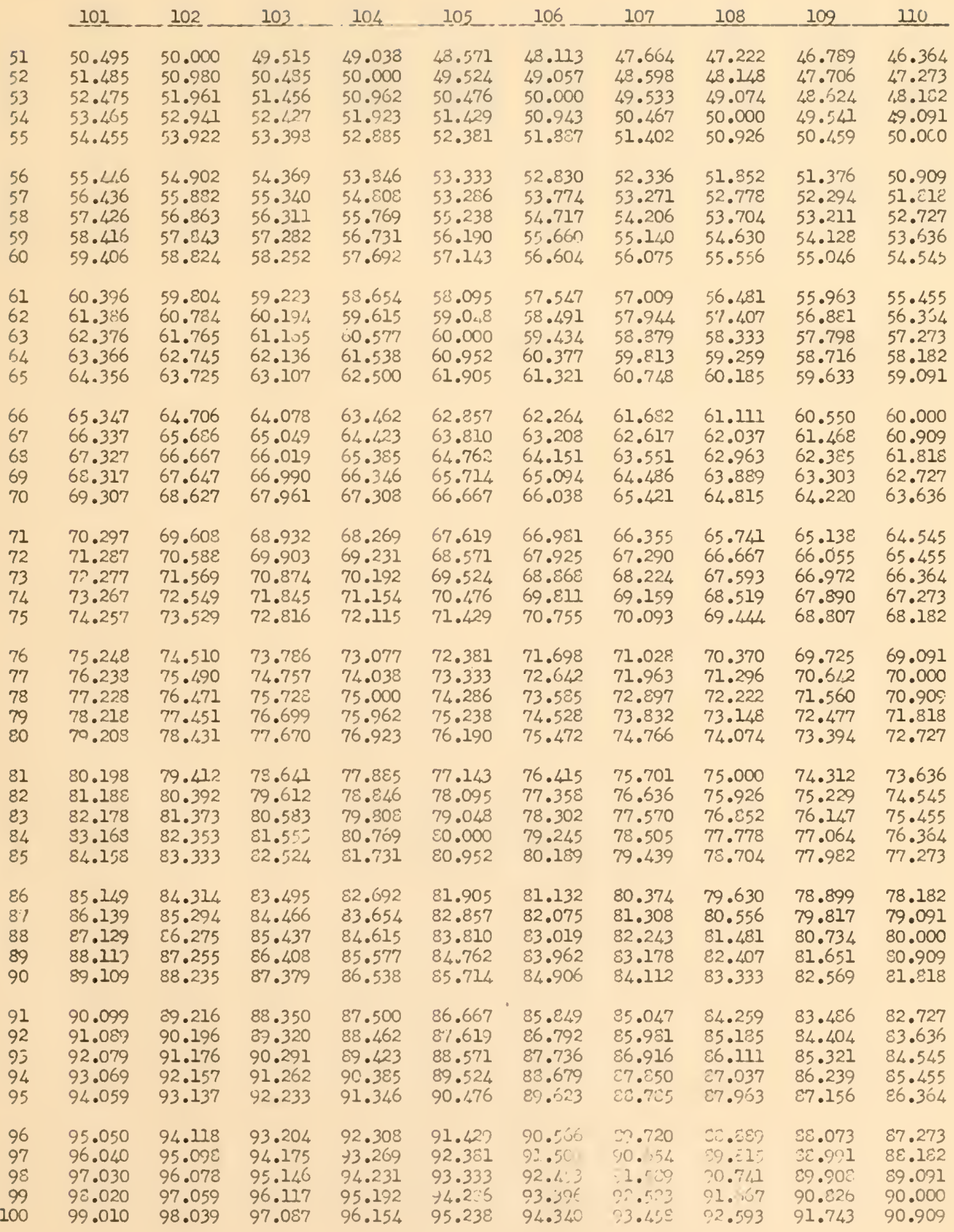


PERCENTAGE, TABLES

\begin{tabular}{|c|c|c|c|c|c|c|c|c|c|c|}
\hline & 101. & 102 & 103 & 104 & 105 & 106 & 107 & 108 & 109 & 111 \\
\hline 101 & 100.000 & 99.020 & 98.058 & 97.115 & 96.190 & 95.283 & 94.393 & 93.519 & 92.661 & 91.818 \\
\hline 02 & - & 100.000 & 99.029 & 98.077 & 97.143 & 96.226 & 95. & 94.444 & 93.578 & 92.727 \\
\hline 103 & - & - & 100.000 & 99.038 & 98.095 & 97.170 & 96.26 & 95.370 & 94.495 & 93.636 \\
\hline 104 & - & - & - & 100.000 & 99.048 & 98.113 & 97.196 & 96.296 & 95.413 & 94.545 \\
\hline 105 & - & --- & -- & -- & 100.000 & 99.057 & 98.131 & 97.222 & 96.330 & 95.455 \\
\hline & - & -- & - & - & - & 100.000 & 99.065 & $98.1 / 8$ & 97.248 & 96.364 \\
\hline & - & - & -- & -- & $\ldots$ & - & 100.000 & 99.074 & 98.165 & 97.273 \\
\hline & - & -- & -- & -- & --- & -- & -. & 100.000 & 99.083 & 98.132 \\
\hline 1 & -- & $\ldots$ & - & --- & --- & - & -- & _. & 100.000 & 99.091 \\
\hline 110 & - & - & -- & --- & -- & --- & -- & --- & - & 100.000 \\
\hline
\end{tabular}




$$
\text { - }
$$




\begin{tabular}{|c|c|c|c|c|c|c|c|c|c|c|}
\hline & 111 & 112 & 113 & 114 & 115 & 116 & 117 & 118 & 119 & 120 \\
\hline $\begin{array}{l}1 \\
2 \\
3 \\
4 \\
5\end{array}$ & $\begin{array}{l}0.901 \\
1.802 \\
2.703 \\
3.604 \\
4.505\end{array}$ & $\begin{array}{l}0.893 \\
1.786 \\
2.679 \\
3.571 \\
4.464\end{array}$ & $\begin{array}{l}0.835 \\
1.770 \\
2.655 \\
3.540 \\
4.425\end{array}$ & $\begin{array}{l}0.877 \\
1.754 \\
2.632 \\
3.509 \\
4.386\end{array}$ & $\begin{array}{l}0.870 \\
1.739 \\
2.609 \\
3.478 \\
4.348\end{array}$ & $\begin{array}{l}0.862 \\
1.724 \\
2.586 \\
3.448 \\
4.310\end{array}$ & $\begin{array}{l}0.855 \\
1.709 \\
2.564 \\
3.419 \\
4.274\end{array}$ & $\begin{array}{l}0.847 \\
1.695 \\
2.542 \\
3.390 \\
4.237\end{array}$ & $\begin{array}{l}0.840 \\
1.681 \\
2.521 \\
3.361 \\
4.202\end{array}$ & $\begin{array}{l}0.833 \\
1.667 \\
2.500 \\
3.333 \\
4.167\end{array}$ \\
\hline $\begin{array}{r}6 \\
7 \\
8 \\
9 \\
10\end{array}$ & $\begin{array}{l}5.405 \\
6.306 \\
7.207 \\
8.108 \\
9.009\end{array}$ & $\begin{array}{l}5.357 \\
6.250 \\
7.143 \\
8.036 \\
8.929\end{array}$ & $\begin{array}{l}5.310 \\
6.195 \\
7.080 \\
7.965 \\
8.850\end{array}$ & $\begin{array}{l}5.263 \\
6.140 \\
7.018 \\
7.895 \\
8.772\end{array}$ & $\begin{array}{l}5.217 \\
6.087 \\
6.957 \\
7.826 \\
3.696\end{array}$ & $\begin{array}{l}5.172 \\
6.234 \\
6.897 \\
7.759 \\
0.621\end{array}$ & $\begin{array}{l}5.128 \\
5.983 \\
6.838 \\
7.692 \\
8.547\end{array}$ & $\begin{array}{l}5.085 \\
5.932 \\
6.780 \\
7.627 \\
8.475\end{array}$ & $\begin{array}{l}5.042 \\
5.882 \\
6.723 \\
7.563 \\
8.403\end{array}$ & $\begin{array}{l}5.700 \\
5.83 \\
6.65 \\
7.500 \\
8.33\end{array}$ \\
\hline $\begin{array}{l}13 \\
14 \\
15\end{array}$ & $\begin{array}{r}9.910 \\
10.811 \\
11.712 \\
12.613 \\
13.514\end{array}$ & $\begin{array}{r}9.821 \\
10.714 \\
11.607 \\
12.500 \\
13.393\end{array}$ & $\begin{array}{r}9.735 \\
10.619 \\
11.504 \\
12.389 \\
13.274\end{array}$ & $\begin{array}{r}9.649 \\
10.526 \\
11.404 \\
12.261 \\
13.158\end{array}$ & $\begin{array}{r}9.565 \\
10.435 \\
11.304 \\
12.174 \\
13.243\end{array}$ & $\begin{array}{r}9.483 \\
10.345 \\
11.207 \\
12.069 \\
12.931\end{array}$ & $\begin{array}{r}9.402 \\
10.256 \\
11.111 \\
11.266 \\
12.821\end{array}$ & $\begin{array}{r}9.322 \\
10.169 \\
11.017 \\
11.864 \\
12.712\end{array}$ & $\begin{array}{r}9.244 \\
10.084 \\
10.924 \\
11.765 \\
12.605\end{array}$ & $\begin{array}{r}9.16 \\
10.000 \\
10.83 \\
11.66 \\
12.500\end{array}$ \\
\hline $\begin{array}{l}17 \\
18 \\
19 \\
20\end{array}$ & $\begin{array}{l}14.414 \\
15.315 \\
16.216 \\
17.117 \\
18.018\end{array}$ & $\begin{array}{l}14.286 \\
15.179 \\
16.071 \\
16.964 \\
17.857\end{array}$ & $\begin{array}{l}14.159 \\
15.044 \\
15.929 \\
16.814 \\
17.699\end{array}$ & $\begin{array}{l}14.035 \\
14.912 \\
15.789 \\
16.667 \\
17.544\end{array}$ & $\begin{array}{l}13.913 \\
14.783 \\
15.552 \\
16.522 \\
17.391\end{array}$ & $\begin{array}{l}13.793 \\
14.655 \\
15.517 \\
16.379 \\
17.241\end{array}$ & $\begin{array}{l}13.675 \\
14.530 \\
15.385 \\
16.239 \\
17.094\end{array}$ & $\begin{array}{l}13.559 \\
14.407 \\
15.254 \\
16.102 \\
16.949\end{array}$ & $\begin{array}{l}13.445 \\
14.286 \\
15.126 \\
15.966 \\
16.807\end{array}$ & $\begin{array}{l}13.33 \\
14.16 \\
15.00 \\
15.83 \\
16.66\end{array}$ \\
\hline $\begin{array}{l}21 \\
22 \\
23 \\
24 \\
25\end{array}$ & $\begin{array}{l}18.929 \\
19.820 \\
20.721 \\
21.622 \\
22.523\end{array}$ & $\begin{array}{l}18.750 \\
19.643 \\
20.536 \\
21.429 \\
22.321\end{array}$ & $\begin{array}{l}18.534 \\
19.469 \\
20.354 \\
21.239 \\
22.124\end{array}$ & $\begin{array}{l}18.421 \\
19.298 \\
20.175 \\
21.053 \\
21.930\end{array}$ & $\begin{array}{l}18.261 \\
19.130 \\
20.000 \\
20.870 \\
21.739\end{array}$ & $\begin{array}{l}18.173 \\
18.966 \\
19.826 \\
20.690 \\
21.552\end{array}$ & $\begin{array}{l}17.949 \\
18.803 \\
19.658 \\
20.513 \\
21.368\end{array}$ & $\begin{array}{l}17.797 \\
18.644 \\
19.492 \\
20.339 \\
21.186\end{array}$ & $\begin{array}{l}17.647 \\
18.487 \\
19.328 \\
20.168 \\
21.008\end{array}$ & $\begin{array}{l}17.50 \\
18.33 \\
19.16 \\
20.00 \\
20.83\end{array}$ \\
\hline $\begin{array}{l}26 \\
27 \\
28 \\
29 \\
30\end{array}$ & $\begin{array}{l}23.423 \\
24.324 \\
25.225 \\
26.126 \\
27.027\end{array}$ & $\begin{array}{l}23.214 \\
24.107 \\
25.000 \\
25.893 \\
26.786\end{array}$ & $\begin{array}{l}23.009 \\
23.894 \\
24.779 \\
25.664 \\
26.549\end{array}$ & $\begin{array}{l}22.807 \\
23.684 \\
24.561 \\
25.439 \\
26.316\end{array}$ & $\begin{array}{l}22.609 \\
23.478 \\
24.348 \\
25.217 \\
26.087\end{array}$ & $\begin{array}{l}22.414 \\
23.276 \\
24.138 \\
25.000 \\
25.862\end{array}$ & $\begin{array}{l}22.222 \\
23.077 \\
23.932 \\
24.736 \\
25.541\end{array}$ & $\begin{array}{l}22.034 \\
22.881 \\
23.729 \\
24.576 \\
25.424\end{array}$ & $\begin{array}{l}21.849 \\
22.689 \\
23.529 \\
24.370 \\
25.210\end{array}$ & $\begin{array}{l}21.66 \\
22.50 \\
23.33 \\
24.16 \\
25.00\end{array}$ \\
\hline $\begin{array}{l}21 \\
32 \\
33 \\
34 \\
35\end{array}$ & $\begin{array}{l}27.928 \\
28.829 \\
29.730 \\
30.631 \\
31.532\end{array}$ & $\begin{array}{l}27.679 \\
28.571 \\
29.464 \\
30.357 \\
31.250\end{array}$ & $\begin{array}{l}27.434 \\
28.319 \\
29.204 \\
30.088 \\
30.973\end{array}$ & $\begin{array}{l}27.193 \\
28.070 \\
28.947 \\
29.825 \\
30.702\end{array}$ & $\begin{array}{l}26.957 \\
27.826 \\
28.696 \\
29.565 \\
30.435\end{array}$ & $\begin{array}{l}26.724 \\
27.586 \\
28.448 \\
29.310 \\
30.172\end{array}$ & $\begin{array}{l}26.496 \\
27.350 \\
28.205 \\
29.060 \\
29.915\end{array}$ & $\begin{array}{l}26.271 \\
27.119 \\
27.966 \\
28.814 \\
29.661\end{array}$ & $\begin{array}{l}26.050 \\
26.891 \\
27.731 \\
28.571 \\
29.412\end{array}$ & $\begin{array}{l}25.83 \\
26.66 \\
27.50 \\
28.33 \\
29.16\end{array}$ \\
\hline $\begin{array}{l}36 \\
37 \\
38 \\
39 \\
40\end{array}$ & $\begin{array}{l}32.432 \\
33.333 \\
34.234 \\
35.135 \\
36.036\end{array}$ & $\begin{array}{l}32.143 \\
33.036 \\
33.929 \\
34.821 \\
35.714\end{array}$ & $\begin{array}{l}31.858 \\
32.743 \\
33.628 \\
34.513 \\
35.398\end{array}$ & $\begin{array}{l}31.579 \\
32.456 \\
33.333 \\
34.211 \\
35.086\end{array}$ & $\begin{array}{l}31.304 \\
32.174 \\
33.043 \\
33.913 \\
34.783\end{array}$ & $\begin{array}{l}31.034 \\
31.097 \\
32.759 \\
33.621 \\
34.483\end{array}$ & $\begin{array}{l}30.769 \\
31.624 \\
32.479 \\
33.333 \\
34.183\end{array}$ & $\begin{array}{l}30.508 \\
31.356 \\
32.203 \\
33.051 \\
33.898\end{array}$ & $\begin{array}{l}30.252 \\
31.092 \\
31.933 \\
32.773 \\
33.613\end{array}$ & $\begin{array}{l}30.00 \\
30.83 \\
31.66 \\
32.50 \\
33.33\end{array}$ \\
\hline $\begin{array}{l}42 \\
43 \\
44 \\
45\end{array}$ & $\begin{array}{l}36.937 \\
37.838 \\
38.739 \\
39.640 \\
40.541\end{array}$ & $\begin{array}{l}36.607 \\
37.500 \\
38.393 \\
39.286 \\
40.179\end{array}$ & $\begin{array}{l}36.283 \\
37.168 \\
38.053 \\
38.938 \\
39.823\end{array}$ & $\begin{array}{l}35.965 \\
36.842 \\
37.719 \\
38.596 \\
39.474\end{array}$ & $\begin{array}{l}35.552 \\
36.522 \\
37.391 \\
38.261 \\
39.130\end{array}$ & $\begin{array}{l}35.345 \\
36.207 \\
37.009 \\
37.931 \\
38.793\end{array}$ & $\begin{array}{l}35.043 \\
35.897 \\
36.752 \\
37.607 \\
38.462\end{array}$ & $\begin{array}{l}34.746 \\
35.593 \\
36.441 \\
37.288 \\
38.136\end{array}$ & $\begin{array}{l}34.454 \\
35.294 \\
36.134 \\
36.975 \\
37.815\end{array}$ & $\begin{array}{l}34.16 \\
35.00 \\
35.83 \\
36.66 \\
37.50\end{array}$ \\
\hline $\begin{array}{l}46 \\
47 \\
48 \\
49\end{array}$ & $\begin{array}{l}41.441 \\
42.342 \\
43.243 \\
44.144 \\
45.045\end{array}$ & $\begin{array}{l}41.071 \\
41.964 \\
42.857 \\
43.750 \\
44.643\end{array}$ & $\begin{array}{l}40.708 \\
41.593 \\
42.478 \\
43.363 \\
44.248\end{array}$ & $\begin{array}{l}40.351 \\
41.228 \\
42.105 \\
42.982 \\
43.860\end{array}$ & $\begin{array}{l}40.000 \\
40.870 \\
41.739 \\
42.609 \\
43.478\end{array}$ & $\begin{array}{l}39.655 \\
40.517 \\
41.379 \\
42.241 \\
43.103\end{array}$ & $\begin{array}{l}39.316 \\
40.171 \\
41.026 \\
41.880 \\
42.735\end{array}$ & $\begin{array}{l}38.953 \\
39.830 \\
40.678 \\
41.525 \\
42.373\end{array}$ & $\begin{array}{l}38.655 \\
39.496 \\
40.336 \\
41.176 \\
42.017\end{array}$ & $\begin{array}{l}38.33 \\
39.16 \\
40.00 \\
40.83 \\
41.66\end{array}$ \\
\hline
\end{tabular}





\begin{tabular}{|c|c|c|c|c|c|c|c|c|c|c|}
\hline & 111 & 112 & 113 & 114 & 115 & 116 & 117 & 118 & 112 & 120 \\
\hline & $\begin{array}{l}45.946 \\
46.817 \\
47.748 \\
48.649 \\
49.550\end{array}$ & $\begin{array}{l}45.536 \\
46.429 \\
47.321 \\
48.214 \\
49.107\end{array}$ & $\begin{array}{l}45.133 \\
46.015 \\
16.903 \\
47.788 \\
48.673\end{array}$ & $\begin{array}{l}44.737 \\
45.614 \\
46.491 \\
47.368 \\
48.246\end{array}$ & $\begin{array}{l}44.348 \\
45.217 \\
46.087 \\
46.957 \\
47.826\end{array}$ & $\begin{array}{l}43.966 \\
44.828 \\
45.690 \\
46.552 \\
47.414\end{array}$ & $\begin{array}{l}43.590 \\
44_{4} .444 \\
45.299 \\
46.154 \\
47.009\end{array}$ & $\begin{array}{l}43.220 \\
14.068 \\
4.915 \\
45.763 \\
46.610\end{array}$ & $\begin{array}{l}42.857 \\
43.697 \\
44.538 \\
45.378 \\
46.216\end{array}$ & $\begin{array}{l}12.500 \\
43.333 \\
44.167 \\
45.000 \\
45.833\end{array}$ \\
\hline & $\begin{array}{l}50.450 \\
51.351 \\
52.252 \\
53.153 \\
54.054\end{array}$ & $\begin{array}{l}50.000 \\
50.893 \\
51.786 \\
52.679 \\
53.571\end{array}$ & $\begin{array}{l}49.558 \\
50.41,2 \\
51.327 \\
52.212 \\
53.097\end{array}$ & $\begin{array}{l}49.123 \\
50.000 \\
50.877 \\
51.754 \\
52.632\end{array}$ & $\begin{array}{l}48.696 \\
49.565 \\
50.1,35 \\
51.304 \\
52.174\end{array}$ & $\begin{array}{l}48.276 \\
49.138 \\
50.000 \\
50.862 \\
51.724\end{array}$ & $\begin{array}{l}47.863 \\
48.718 \\
49.573 \\
50.427 \\
51.282\end{array}$ & $\begin{array}{l}47.458 \\
48.305 \\
49.153 \\
50.000 \\
50.847\end{array}$ & $\begin{array}{l}47.059 \\
47.899 \\
48.739 \\
49.580 \\
50.420\end{array}$ & $\begin{array}{l}46.667 \\
47.500 \\
48.333 \\
49.167 \\
50.000\end{array}$ \\
\hline 64 & $\begin{array}{l}54.955 \\
55.856 \\
56.757 \\
57.658 \\
58.559\end{array}$ & $\begin{array}{l}54.464 \\
5 \div .357 \\
56.250 \\
57.143 \\
58.036\end{array}$ & $\begin{array}{l}53.982 \\
54.567 \\
55.752 \\
56.637 \\
57.522\end{array}$ & $\begin{array}{l}53.509 \\
54.386 \\
55.263 \\
56.140 \\
57.018\end{array}$ & $\begin{array}{l}53.043 \\
53.913 \\
54.783 \\
55.652 \\
56.522\end{array}$ & $\begin{array}{l}52.586 \\
53.11_{1} \\
54.310 \\
55.172 \\
56.034\end{array}$ & & & $\begin{array}{l}51.261 \\
52.1 .01 \\
52.941 \\
53.782 \\
54.627\end{array}$ & $\begin{array}{l}50.833 \\
51.567 \\
52.500 \\
53.333 \\
54.167\end{array}$ \\
\hline $\begin{array}{l}66 \\
67 \\
68 \\
69 \\
70\end{array}$ & $\begin{array}{l}59.459 \\
60.360 \\
61.261 \\
62.162 \\
63.063\end{array}$ & $\begin{array}{l}58.929 \\
59.821 \\
60.714 \\
61.607 \\
62.500\end{array}$ & $\begin{array}{l}58.407 \\
59.292 \\
60.177 \\
61.062 \\
51.947\end{array}$ & $\begin{array}{l}57.895 \\
58.772 \\
59.649 \\
50.526 \\
61.404\end{array}$ & $\begin{array}{l}57.391 \\
58.261 \\
59.130 \\
60.000 \\
60.870\end{array}$ & $\begin{array}{l}56.897 \\
57.759 \\
58.621 \\
59.483 \\
60.345\end{array}$ & & & $\begin{array}{l}55.462 \\
56.303 \\
57.143 \\
57.983 \\
58.824\end{array}$ & $\begin{array}{l}55.000 \\
55.033 \\
56.657 \\
57.500 \\
58.333\end{array}$ \\
\hline $\begin{array}{l}71 \\
72 \\
73 \\
74 \\
75\end{array}$ & $\begin{array}{l}63.964 \\
64.865 \\
65.766 \\
66.667 \\
67.563\end{array}$ & $\begin{array}{l}63.393 \\
64.286 \\
65.179 \\
66.071 \\
66.904\end{array}$ & $\begin{array}{l}62.832 \\
63.717 \\
64.602 \\
65.457 \\
65.372\end{array}$ & $\begin{array}{l}62.281 \\
63.158 \\
64.035 \\
64.912 \\
65.789\end{array}$ & $\begin{array}{l}61.739 \\
62.609 \\
63.478 \\
64.348 \\
65.217\end{array}$ & $\begin{array}{l}61.207 \\
62.069 \\
62.931 \\
63.793 \\
64.655\end{array}$ & & & $\begin{array}{l}59.664 \\
60.504 \\
61.3145 \\
62.185 \\
63.025\end{array}$ & $\begin{array}{l}59.167 \\
60.000 \\
60.833 \\
61.667 \\
62.500\end{array}$ \\
\hline $\begin{array}{l}76 \\
77 \\
78 \\
79 \\
80\end{array}$ & $\begin{array}{l}6 \varepsilon .465 \\
69.369 \\
70.270 \\
71.171 \\
72.072\end{array}$ & $\begin{array}{l}67.857 \\
68.750 \\
69.643 \\
70.536 \\
71.429\end{array}$ & $\begin{array}{l}67.257 \\
68.142 \\
69.027 \\
69.912 \\
70.796\end{array}$ & $\begin{array}{l}66.667 \\
67.544 \\
63.121 \\
69.298 \\
70.175\end{array}$ & $\begin{array}{l}66.087 \\
66.957 \\
67.826 \\
68.696 \\
69.565\end{array}$ & $\begin{array}{l}65.517 \\
66.379 \\
67.241 \\
68.103 \\
68.966\end{array}$ & $\begin{array}{l}4.957 \\
5.812 \\
6.667 \\
7.521 \\
8.376\end{array}$ & & $\begin{array}{l}63.266 \\
64.706 \\
65.546 \\
66.387 \\
67.227\end{array}$ & $\begin{array}{l}63.333 \\
64.167 \\
65.000 \\
65.833 \\
66.667\end{array}$ \\
\hline $\begin{array}{l}81 \\
82 \\
83 \\
84 \\
85\end{array}$ & $\begin{array}{l}72.973 \\
73.874 \\
74.775 \\
75.676 \\
76.577\end{array}$ & $\begin{array}{l}72.321 \\
73.214 \\
74.107 \\
75.000 \\
75.893\end{array}$ & & & & & & & & $\begin{array}{l}67.500 \\
63.333 \\
69.167 \\
70.000 \\
70.833\end{array}$ \\
\hline $\begin{array}{l}86 \\
87 \\
88 \\
89 \\
90\end{array}$ & & & & & & & & & & $\begin{array}{l}71.667 \\
72.500 \\
73.333 \\
74.167 \\
75.000\end{array}$ \\
\hline $\begin{array}{l}91 \\
92 \\
93 \\
94\end{array}$ & $\begin{array}{l}81.982 \\
82.883 \\
83.784 \\
84.685 \\
85.586\end{array}$ & $\begin{array}{l}81.250 \\
82.143 \\
83.036 \\
83.929 \\
84.821\end{array}$ & $\begin{array}{l}80.5 .31 \\
81.416 \\
82.301 \\
83.196 \\
84.071\end{array}$ & $\begin{array}{l}79.82 .5 \\
80.702 \\
81.579 \\
82.456 \\
83.333\end{array}$ & & & & & $\begin{array}{l}76.471 \\
77.311 \\
78.151 \\
78.992 \\
79.832\end{array}$ & $\begin{array}{l}75.833 \\
76.667 \\
77.500 \\
7 \varepsilon .333 \\
79.167\end{array}$ \\
\hline 96 & $\begin{array}{l}86.486 \\
87.307 \\
88.285 \\
89.189 \\
90.090\end{array}$ & $\begin{array}{l}86.607 \\
87.500 \\
88.393 \\
89.286\end{array}$ & $\begin{array}{l}84.956 \\
85.841 \\
86.726 \\
87.611 \\
88.496\end{array}$ & $\begin{array}{l}\therefore 6 .: \angle 2 \\
87.715\end{array}$ & $\begin{array}{l}8 / .345 \\
55.217 \\
86.057 \\
66.957\end{array}$ & $\begin{array}{l}83.621 \\
84.6 .23 \\
85.345 \\
86.207\end{array}$ & $\begin{array}{l}82.906 \\
83.761 \\
64.615 \\
25.470\end{array}$ & $\begin{array}{l}81.356 \\
82.203 \\
83.051 \\
83.898 \\
84.746\end{array}$ & $\begin{array}{l}80.672 \\
81.513 \\
82.353 \\
83.193 \\
84.031\end{array}$ & $\begin{array}{l}80.000 \\
80.833 \\
81.667 \\
82.500 \\
83.333\end{array}$ \\
\hline
\end{tabular}



PERCENTAGE TABLES

\begin{tabular}{|c|c|c|c|c|c|c|c|c|c|c|}
\hline & 111 & 112 & 213 & 114 & 115 & -116 & 117 & 218 & 112 & 120 \\
\hline $\begin{array}{l}101 \\
102 \\
103 \\
104 \\
105\end{array}$ & $\begin{array}{l}90.991 \\
91.892 \\
92.793 \\
93.694 \\
94.595\end{array}$ & $\begin{array}{l}90.179 \\
91.071 \\
91.964 \\
92.857 \\
93.750\end{array}$ & $\begin{array}{l}89.381 \\
90.265 \\
91.150 \\
92.035 \\
92.920\end{array}$ & $\begin{array}{l}88.596 \\
89.474 \\
90.351 \\
91.228 \\
92.105\end{array}$ & $\begin{array}{l}87.826 \\
88.696 \\
89.565 \\
90.435 \\
91.304\end{array}$ & $\begin{array}{l}87.069 \\
87.931 \\
88.793 \\
89.655 \\
90.517\end{array}$ & $\begin{array}{l}86.325 \\
87.179 \\
88.034 \\
88.889 \\
89.744\end{array}$ & $\begin{array}{l}85.593 \\
86.441 \\
87.288 \\
88.136 \\
88.983\end{array}$ & $\begin{array}{l}84.874 \\
85.714 \\
86.555 \\
87.395 \\
88.235\end{array}$ & $\begin{array}{l}84.167 \\
85.000 \\
85.833 \\
86.667 \\
87.500\end{array}$ \\
\hline $\begin{array}{l}106 \\
107 \\
108 \\
109 \\
110\end{array}$ & $\begin{array}{l}95.495 \\
96.396 \\
97.297 \\
98.198 \\
99.099\end{array}$ & $\begin{array}{l}94.643 \\
95.536 \\
96.429 \\
97.321 \\
98.214\end{array}$ & $\begin{array}{l}93.805 \\
94.690 \\
95.575 \\
96.460 \\
97.345\end{array}$ & $\begin{array}{l}92.982 \\
93.860 \\
94.737 \\
95.614 \\
96.491\end{array}$ & $\begin{array}{l}92.174 \\
93.043 \\
93.913 \\
94.783 \\
95.652\end{array}$ & $\begin{array}{l}91.379 \\
92.247 \\
93.103 \\
93.966 \\
94.828\end{array}$ & $\begin{array}{l}90.598 \\
91.453 \\
92.308 \\
93.162 \\
94.017\end{array}$ & $\begin{array}{l}89.831 \\
90.678 \\
91.525 \\
92.373 \\
93.220\end{array}$ & $\begin{array}{l}89.076 \\
89.916 \\
90.756 \\
91.597 \\
92.437\end{array}$ & $\begin{array}{l}88.333 \\
89.167 \\
90.000 \\
90.833 \\
91.667\end{array}$ \\
\hline $\begin{array}{l}111 \\
112 \\
113 \\
114 \\
115\end{array}$ & ${ }^{100.000}=$ & $\begin{array}{c}99.107 \\
100.000 \\
-- \\
--\end{array}$ & $\begin{array}{r}98.230 \\
99.125 \\
100.000 \\
-\end{array}$ & $\begin{array}{r}97.368 \\
98.246 \\
99.123 \\
100.000 \\
\end{array}$ & $\begin{array}{r}96.522 \\
97.391 \\
98.261 \\
99.130 \\
100.000\end{array}$ & $\begin{array}{l}95.690 \\
96.552 \\
97.414 \\
98.276 \\
99.138\end{array}$ & $\begin{array}{l}94.872 \\
95.726 \\
96.581 \\
97.436 \\
98.291\end{array}$ & $\begin{array}{l}94.068 \\
94.915 \\
95.763 \\
96.610 \\
97.458\end{array}$ & $\begin{array}{l}93.277 \\
94.118 \\
94.958 \\
95.798 \\
96.639\end{array}$ & $\begin{array}{l}92.500 \\
93.333 \\
91.167 \\
95.000 \\
95.833\end{array}$ \\
\hline $\begin{array}{l}116 \\
117 \\
118 \\
119\end{array}$ & $\overline{-}$ & $\overline{-}$ & 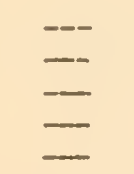 & $\overline{-}$ & $\bar{m}$ & $\frac{100.000}{-}$ & $\begin{array}{r}99.145 \\
100.000 \\
-\end{array}$ & $\begin{array}{r}98.305 \\
99.153 \\
100.000 \\
=\end{array}$ & $\begin{array}{r}97.249 \\
95.329 \\
99.160 \\
100.000 \\
\end{array}$ & $\begin{array}{r}9.867 \\
97 \% .500 \\
98.333 \\
99.167 \\
200.000\end{array}$ \\
\hline
\end{tabular}





\begin{tabular}{|c|c|c|c|c|c|c|c|c|c|}
\hline 121 & 122 & 123 & 124 & 125 & 126 & 127 & 128 & 129 & 130 \\
\hline $\begin{array}{l}0.826 \\
1.653 \\
2.479 \\
3.306 \\
4.132\end{array}$ & $\begin{array}{l}0.320 \\
1.639 \\
2.459 \\
3.279 \\
4.098\end{array}$ & $\begin{array}{l}0.813 \\
1.626 \\
2.439 \\
3.252 \\
4.065\end{array}$ & $\begin{array}{l}0.806 \\
1.013 \\
2.419 \\
3.226 \\
4.032\end{array}$ & $\begin{array}{l}0.800 \\
1.600 \\
2.400 \\
3.200 \\
4.000\end{array}$ & $\begin{array}{l}0.794 \\
1.587 \\
2.331 \\
3.175 \\
3.968\end{array}$ & $\begin{array}{l}0.787 \\
1.575 \\
2.362 \\
3.150 \\
3.937\end{array}$ & $\begin{array}{l}0.731 \\
1.562 \\
2.344 \\
3.125 \\
3.905\end{array}$ & $\begin{array}{l}0.775 \\
1.550 \\
2.326 \\
3.171 \\
3.876\end{array}$ & $\begin{array}{l}0.76 \\
1.53 \\
2.37 \\
3.07 \\
3.84\end{array}$ \\
\hline $\begin{array}{l}4.959 \\
5.785 \\
6.612 \\
7.433 \\
8.264\end{array}$ & $\begin{array}{l}4.918 \\
5.738 \\
0.557 \\
7.377 \\
3.197\end{array}$ & $\begin{array}{l}4.878 \\
5.691 \\
6.504 \\
7.317 \\
3.130\end{array}$ & $\begin{array}{l}4.839 \\
5.645 \\
6.452 \\
7.258 \\
8.065\end{array}$ & $\begin{array}{l}4.500 \\
5.600 \\
6.400 \\
7.200 \\
3.000\end{array}$ & $\begin{array}{l}4.762 \\
5.556 \\
6.349 \\
7.143 \\
7.937\end{array}$ & $\begin{array}{l}4.724 \\
5.512 \\
6.299 \\
7.087 \\
7.874\end{array}$ & $\begin{array}{l}4.608 \\
5.469 \\
6.250 \\
7.031 \\
7.012\end{array}$ & $\begin{array}{l}4.651 \\
5.426 \\
6.202 \\
6.977 \\
7.752\end{array}$ & $\begin{array}{l}4.01 \\
5.38 \\
6.15 \\
6.92 \\
7.59\end{array}$ \\
\hline $\begin{array}{r}9.091 \\
9.917 \\
10.744 \\
11.570 \\
12.397\end{array}$ & $\begin{array}{r}9.016 \\
9.636 \\
10.656 \\
11.475 \\
12.295\end{array}$ & $\begin{array}{r}8.943 \\
9.756 \\
10.569 \\
11.332 \\
12.195\end{array}$ & $\begin{array}{r}8.871 \\
9.677 \\
10.484 \\
11.290 \\
12.097\end{array}$ & $\begin{array}{r}8.300 \\
9.600 \\
10.400 \\
11.200 \\
12.000\end{array}$ & $\begin{array}{r}8.730 \\
9.524 \\
10.317 \\
11.111 \\
11.905\end{array}$ & $\begin{array}{r}3.561 \\
9.449 \\
10.236 \\
11.024 \\
11.811\end{array}$ & $\begin{array}{r}8.594 \\
9.375 \\
10.156 \\
10.938 \\
11.719\end{array}$ & $\begin{array}{r}8.527 \\
9.302 \\
10.778 \\
10.353 \\
11.328\end{array}$ & $\begin{array}{r}8.46 \\
9.23 \\
10.03 \\
10.76 \\
11.53\end{array}$ \\
\hline $\begin{array}{l}13.223 \\
14.050 \\
14.376 \\
15.702 \\
16.529\end{array}$ & $\begin{array}{l}13.115 \\
13.934 \\
14.754 \\
15.574 \\
10.393\end{array}$ & $\begin{array}{l}13.208 \\
13.821 \\
14.634 \\
15.447 \\
16.260\end{array}$ & $\begin{array}{l}12.903 \\
13.710 \\
14.516 \\
15.323 \\
16.129\end{array}$ & $\begin{array}{l}12.300 \\
13.500 \\
14.40 \\
15.200 \\
16.000\end{array}$ & $\begin{array}{l}12.698 \\
13.492 \\
14.236 \\
15.079 \\
15.873\end{array}$ & $\begin{array}{l}12.598 \\
13.385 \\
14.173 \\
14.951 \\
15.7^{\prime}+3\end{array}$ & $\begin{array}{l}12.200 \\
13.281 \\
14.062 \\
14.0144 \\
15.625\end{array}$ & $\begin{array}{l}12.403 \\
13.173 \\
13.953 \\
14.729 \\
15.504\end{array}$ & $\begin{array}{l}12.32 \\
13.07 \\
13.84 \\
14.51 \\
15.38\end{array}$ \\
\hline $\begin{array}{l}17.355 \\
18.152 \\
19.008 \\
19.835 \\
20.651\end{array}$ & $\begin{array}{l}17.213 \\
18.033 \\
18.852 \\
19.672 \\
20.432\end{array}$ & $\begin{array}{l}17.073 \\
17.086 \\
18.699 \\
19.512 \\
20.325\end{array}$ & $\begin{array}{l}16.935 \\
17.742 \\
18.548 \\
19.355 \\
20.161\end{array}$ & $\begin{array}{l}16.800 \\
17.600 \\
10.400 \\
19.200 \\
20.500\end{array}$ & $\begin{array}{l}15.667 \\
17.450 \\
18.254 \\
19.043 \\
19.841\end{array}$ & $\begin{array}{l}16.535 \\
17.323 \\
13.110 \\
13.898 \\
19.685\end{array}$ & $\begin{array}{l}16.406 \\
17.188 \\
17.969 \\
18.750 \\
19.531\end{array}$ & $\begin{array}{l}16.279 \\
17.054 \\
17.829 \\
18.605 \\
19.330\end{array}$ & $\begin{array}{l}10.15 \\
16.92 \\
17.60 \\
10.46 \\
19.23\end{array}$ \\
\hline $\begin{array}{l}21.488 \\
22.314 \\
23.140 \\
23.967 \\
24.793\end{array}$ & $\begin{array}{l}21.311 \\
22.131 \\
22.951 \\
23.770 \\
24.590\end{array}$ & $\begin{array}{l}21.138 \\
21.951 \\
22.764 \\
23.577 \\
24.390\end{array}$ & $\begin{array}{l}20.968 \\
21.774 \\
22.581 \\
23.387 \\
24.194\end{array}$ & $\begin{array}{l}20.000 \\
21.600 \\
22.400 \\
23.200 \\
24.000\end{array}$ & $\begin{array}{l}20.635 \\
21.429 \\
22.222 \\
23.016 \\
23.310\end{array}$ & $\begin{array}{l}20.472 \\
21.260 \\
22.047 \\
22.835 \\
23.522\end{array}$ & $\begin{array}{l}20.312 \\
21.794 \\
21.875 \\
22.656 \\
23.438\end{array}$ & $\begin{array}{l}20.155 \\
20.930 \\
21.705 \\
22.481 \\
23.256\end{array}$ & $\begin{array}{l}20.9 \\
27.7 \\
21.5 \\
22.3 \\
23.0\end{array}$ \\
\hline $\begin{array}{l}25.620 \\
20.446 \\
27.273 \\
28.099 \\
23.926\end{array}$ & $\begin{array}{l}25.410 \\
26.230 \\
27.049 \\
27.869 \\
28.689\end{array}$ & $\begin{array}{l}25.203 \\
26.016 \\
26.829 \\
27.642 \\
20.455\end{array}$ & $\begin{array}{l}25.0 n 0 \\
25.816 \\
26.613 \\
27.419 \\
28.226\end{array}$ & $\begin{array}{l}24.600 \\
25.500 \\
26.400 \\
27.200 \\
28.000\end{array}$ & $\begin{array}{l}24.503 \\
25.397 \\
26.190 \\
25.984 \\
27.778\end{array}$ & $\begin{array}{l}24.409 \\
25.197 \\
25.934 \\
26.772 \\
27.559\end{array}$ & $\begin{array}{l}24.219 \\
25.000 \\
25.731 \\
26.562 \\
27.344\end{array}$ & $\begin{array}{l}24.731 \\
24.876 \\
25.581 \\
25.357 \\
27.132\end{array}$ & $\begin{array}{l}25.15 \\
26.9\end{array}$ \\
\hline $\begin{array}{l}29.752 \\
30.573 \\
31.475 \\
32.231 \\
33.258\end{array}$ & $\begin{array}{l}29.508 \\
30.320 \\
31.148 \\
31.957 \\
32.787\end{array}$ & $\begin{array}{l}29.268 \\
30.081 \\
30.894 \\
31.707 \\
32.520\end{array}$ & $\begin{array}{l}29.032 \\
29.839 \\
37.645 \\
31.452 \\
32.258\end{array}$ & $\begin{array}{l}26.800 \\
29.500 \\
30.400 \\
31.200 \\
32.700\end{array}$ & $\begin{array}{l}20.571 \\
29.365 \\
30.159 \\
30.952 \\
31.746\end{array}$ & $\begin{array}{l}23.346 \\
29.134 \\
29.921 \\
30: 709 \\
31.496\end{array}$ & $\begin{array}{l}20.125 \\
25.906 \\
29.050 \\
37.459 \\
31.250\end{array}$ & $\begin{array}{l}27.907 \\
20.302 \\
29.457 \\
37.233 \\
31.018\end{array}$ & $\begin{array}{l}27.60 \\
20.45 \\
29.23 \\
30.25 \\
30.76\end{array}$ \\
\hline $\begin{array}{l}33.634 \\
34.711 \\
35.537 \\
36.354 \\
37.170\end{array}$ & $\begin{array}{l}33.607 \\
34.426 \\
35.246 \\
35.056 \\
35.035\end{array}$ & $\begin{array}{l}33.333 \\
34.140 \\
34.359 \\
35.772 \\
36.535\end{array}$ & $\begin{array}{l}33.765 \\
33.871 \\
34.677 \\
35.484 \\
36.290\end{array}$ & $\begin{array}{l}32.300 \\
33.500 \\
34.470 \\
35.200 \\
35.000\end{array}$ & $\begin{array}{l}32.540 \\
33.333 \\
34.127 \\
34.921 \\
35.714\end{array}$ & $\begin{array}{l}32.283 \\
33.071 \\
33.858 \\
34.646 \\
35.433\end{array}$ & $\begin{array}{l}32.031 \\
32.812 \\
33.594 \\
34.375 \\
35.156\end{array}$ & $\begin{array}{l}31.703 \\
32.558 \\
33.323 \\
34.179 \\
34.834\end{array}$ & $\begin{array}{l}31.5 \\
32.3 \\
33.2 \\
33.0 \\
34.5\end{array}$ \\
\hline $\begin{array}{l}30.017 \\
36.343 \\
37.5-9 \\
40.4=6 \\
41.322\end{array}$ & $\begin{array}{l}37.705 \\
30.525 \\
33.344 \\
40.104 \\
40.934\end{array}$ & $\begin{array}{l}37.398 \\
38.211 \\
39.724 \\
37.037 \\
40.650\end{array}$ & $\begin{array}{l}37.0 y 7 \\
37.973 \\
33.710 \\
37.516 \\
40.323\end{array}$ & $\begin{array}{l}36.800 \\
37.007 \\
36.400 \\
39.200 \\
47.000\end{array}$ & $\begin{array}{l}36.508 \\
37.302 \\
33.035 \\
33.839 \\
33.533\end{array}$ & $\begin{array}{l}36.220 \\
37.008 \\
37.795 \\
38.583 \\
39.370\end{array}$ & $\begin{array}{l}35.935 \\
33.719 \\
37.507 \\
33.201 \\
39.252\end{array}$ & $\begin{array}{l}35.659 \\
36.434 \\
37.27 \\
37.854 \\
30.75 n\end{array}$ & $\begin{array}{l}3=0 \\
37=0\end{array}$ \\
\hline
\end{tabular}




\begin{tabular}{|c|c|c|c|c|c|c|c|c|c|c|}
\hline & 221 & 122 & 123 & 124 & 125 & 126 & 127 & 128 & 129 & 130 \\
\hline 51 & 42.149 & 41.803 & 41.463 & 41.129 & 40.800 & 40.476 & 40.157 & 39.844 & 39.535 & 39.231 \\
\hline 52 & 42.975 & 42.623 & 42.276 & 41.935 & 41.600 & 41.270 & 40.945 & 40.625 & 40.310 & 40.000 \\
\hline 53 & 43.802 & 43.443 & 43.089 & 42.742 & 42.400 & 42.063 & 41.732 & 41.406 & $41: 085$ & 40.769 \\
\hline 54 & 44.628 & 44.262 & 43.902 & 43.548 & 43.200 & 42.857 & 42.520 & 42.188 & 41.860 & 41.538 \\
\hline 55 & 45.455 & 45.082 & 44.715 & 44.355 & 44.000 & 43.651 & 43.307 & 42.969 & 42.636 & 42.308 \\
\hline 56 & 46.281 & 45.902 & 45.528 & 45.161 & 44.800 & 44.444 & 44.094 & 43.750 & 43.417 & 43.077 \\
\hline 57 & 47.107 & 46.721 & 46.342 & 45.968 & 45.600 & 45.238 & 44.882 & 44.531 & 44.186 & 43.846 \\
\hline & 47.934 & 47.541 & 47.154 & 46.774 & 46.400 & 46.032 & 45.669 & 45.312 & 44.961 & 44.615 \\
\hline 59 & 48.760 & 48.361 & 47.962 & 47.581 & 47.200 & 46.825 & 46.457 & 46.094 & 45.736 & 45.385 \\
\hline & 49.587 & 49.180 & 48.780 & 48.387 & 48.000 & 47.619 & 47.244 & 46.875 & 46.512 & 46.154 \\
\hline 61 & 50.413 & 50.000 & 49.593 & 49.194 & 48.800 & 48.413 & 48.031 & 47.656 & 47.287 & 46.923 \\
\hline & & 50.820 & 50.407 & 50.000 & 49.600 & .206 & 48.819 & 48.438 & 48.062 & 47.692 \\
\hline 63 & 52.066 & 51.639 & 51.220 & 50.806 & 50.400 & .000 & 49.606 & 49.219 & 48.837 & 48.462 \\
\hline & 52.893 & 52.459 & 52.033 & 51.613 & 51.200 & 50.794 & 50.394 & 50.000 & 49.612 & 49.231 \\
\hline & 53.719 & 53.279 & 52.846 & 52.419 & 52.000 & 51.587 & 51.181 & 50.781 & 50.388 & 50.000 \\
\hline 66 & 54.545 & 54.098 & 53.659 & 53.226 & 52.800 & 52.381 & 51.969 & 51.562 & 51.163 & 50.769 \\
\hline 67 & 55.372 & 54.918 & 54.472 & 54.032 & 53.600 & 175 & .756 & 52.344 & .938 & .538 \\
\hline 68 & 56.198 & 55.738 & 55.285 & 54.839 & 54.400 & 53.968 & 53.543 & 53.125 & 52.713 & 52.308 \\
\hline & 57.025 & 56.557 & 56.098 & 55.645 & 55.200 & 54.762 & 54.331 & 53.906 & 53.488 & 53.077 \\
\hline 70 & 57.851 & 57.377 & 56.911 & 56.452 & 56.000 & 55.556 & 55.118 & 54.688 & 54.264 & 53.846 \\
\hline 71 & 58.678 & 58.197 & 57.724 & 57.258 & 56.800 & 56.349 & 55.906 & 55.469 & 55.039 & 54.615 \\
\hline 72 & 59.504 & 016 & 537 & 065 & 57.600 & .143 & .693 & .250 & .814 & .385 \\
\hline 73 & 60.331 & 59.836 & .350 & 58.871 & 58.400 & 57.937 & 57.480 & 57.031 & 56.589 & 56.154 \\
\hline 74 & 61.157 & 60.656 & 163 & 59.677 & 200 & 730 & 208 & .812 & 57.364 & 56.923 \\
\hline 75 & 61.983 & 61.475 & 60.976 & 60.484 & 60.000 & 59.524 & 59.055 & 58.594 & 58.140 & 57.692 \\
\hline 76 & 62.810 & 62.295 & 61.789 & 61.290 & 60.800 & 60.317 & 843 & 375 & 915 & 58.462 \\
\hline 77 & & & .602 & 097 & 61.600 & 61.111 & 630 & 6 & 690 & .231 \\
\hline 78 & 64.463 & 63.934 & 63.415 & 62.903 & 62.400 & 61.905 & 61.417 & 60.938 & .465 & 0.000 \\
\hline 79 & 65.289 & 64.754 & 64.228 & 63.710 & 63.200 & 698 & 205 & 719 & .260 & 60.769 \\
\hline 80 & 66.116 & 65.574 & 65.041 & 64.516 & 64.000 & 63.492 & 62.992 & 62.500 & 62.016 & 61.538 \\
\hline 81 & 66.942 & 66.393 & 65.854 & 65.323 & 800 & 64.286 & 63.780 & 63.281 & 62.791 & 62.308 \\
\hline 82 & 67.769 & 67.213 & 66.667 & 66.129 & 65.600 & .079 & 64.567 & 64.062 & .566 & .077 \\
\hline 83 & 68.595 & 68.033 & 67.480 & 66.935 & 66.400 & 65.873 & 65.354 & 64.844 & 64.341 & 63.846 \\
\hline 84 & 69.421 & 852 & 293 & & 200 & .667 & .142 & .625 & .116 & 64.615 \\
\hline 85 & 70.248 & 69.672 & 69.106 & 68.548 & 68.000 & 67.460 & 66.929 & 66.406 & 65.891 & 65.385 \\
\hline 86 & 71.074 & 70.492 & 69.919 & 69.355 & 800 & & 67.717 & 67.188 & 667 & 154 \\
\hline 87 & 71,901 & ..311 & .732 & 0.161 & 69.600 & & & 969 & 442 & .923 \\
\hline 88 & 72.727 & 2.131 & .545 & .968 & 70.400 & & .291 & .750 & .217 & 67.692 \\
\hline 89 & 73.554 & 72.951 & & 1.774 & 71.200 & & & & .992 & 68.462 \\
\hline 90 & 74.380 & 73.770 & 73.171 & 72.581 & 72.000 & 71.429 & 70.866 & 70.312 & 69.767 & 69.231 \\
\hline 91 & 75.207 & 590 & 73.984 & 73.387 & 800 & & 71 & & & \\
\hline 92 & & & & 94 & 600 & & & & 71.318 & 70.769 \\
\hline 93 & 76.860 & 76.230 & & & & & & 72.656 & 72.093 & 71.538 \\
\hline 94 & 77.686 & & 76.423 & & & 603 & & & 72.868 & 72.308 \\
\hline 95 & 78.512 & 77.869 & 77.236 & 76.613 & 76.000 & 75.397 & 74.803 & 74.219 & 73.643 & 73.077 \\
\hline 96 & 79.339 & 78.689 & 78.049 & 77.419 & 76.800 & 76.190 & & 75.000 & 74.419 & 73.846 \\
\hline 97 & 80.165 & & 78.862 & 78.226 & 77.600 & 984 & 76.378 & 75.781 & 75.194 & 74.615 \\
\hline & & & & & 78.400 & & & 76.562 & 75.969 & 75.385 \\
\hline 99 & 81.818 & 81.148 & & 79.839 & 79.200 & 78.571 & 77.953 & 77.344 & 76.744 & 76.154 \\
\hline 100 & 82.645 & 81.967 & 81.301 & 80.645 & 80.000 & 79.365 & 78.740 & 78.125 & 77.519 & 76.923 \\
\hline
\end{tabular}




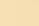


PERCENTAGE TABLES

$\begin{array}{llllllllll}121 & 122 & 123 & 124 & 125 & 126 & 127 & 128 & 129 & 129\end{array}$

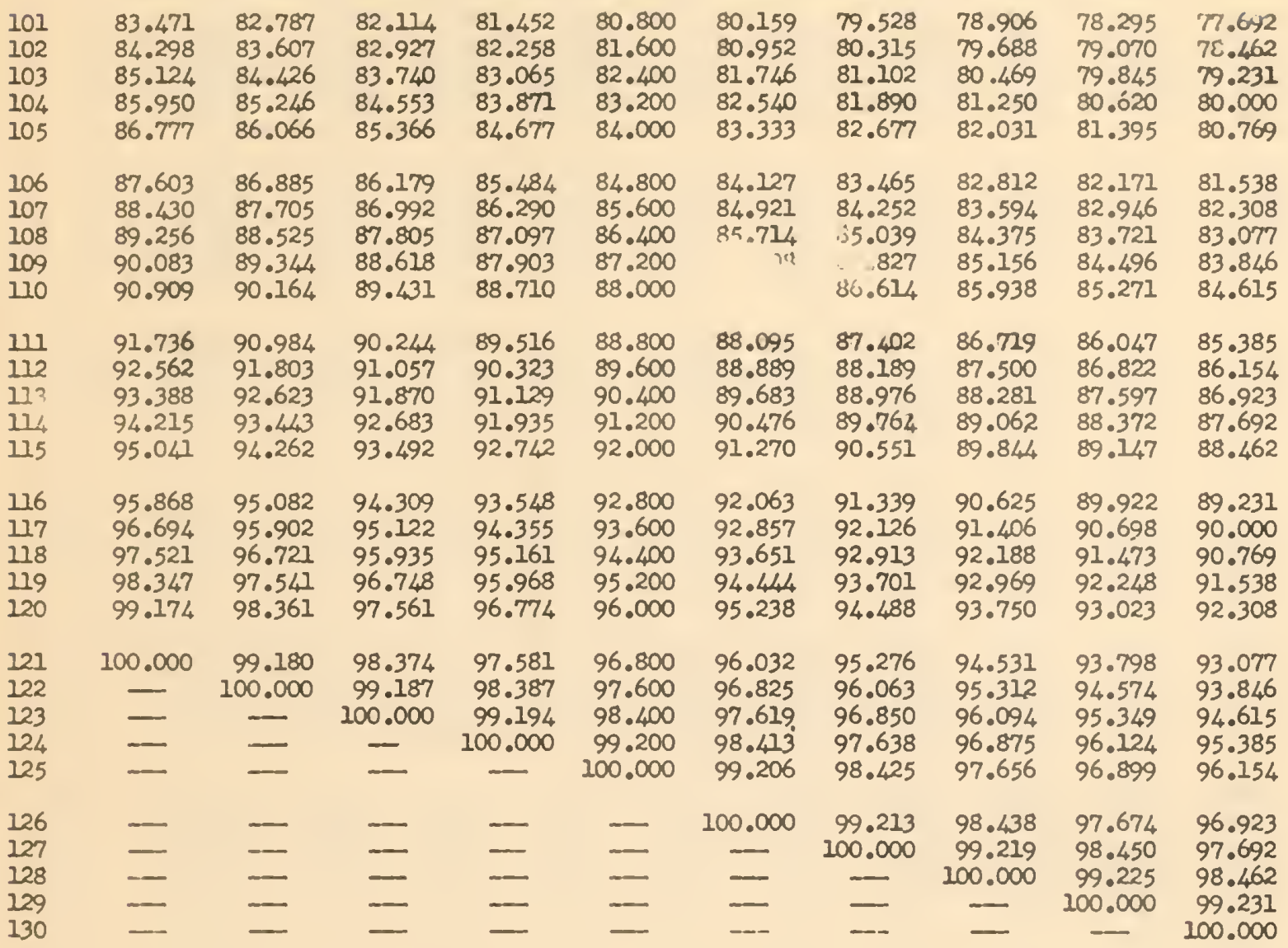



0.763

1.527

0.758

$$
2.290
$$

1.515

0.752

3.053

2.273

1.504

- 0.746

2.256

3.817

3.788

3.008

3.759

4.580

4.545

5.344

6.107

5.303

4.511

6.870

6.061

7.634

6.818

5.263

6.015

6.767

7.519

8.397

8.333

9.160

9.091

12

13

14

9.924

9.848

10.587

10.606

8.271

9.023

9.774

10.526

$11 . / 50$

11.364

11.278

12.214

12.12 .1

12.030

12.977

12.879

12.782

13.534

14.504

13.636

14.286

15.267

15.152

15.038

16.031

15.909

16.794

16.667

15.789

16.541

17.557

17.424

17.293

18.321

15.182

18.045

19.084

18.939

18.797

$19.847 \quad 17.697$

$20.611 \quad 20.455$

21.374

21.212

19.549

20.301

21.053

21.805

13721.970

22.556

$23.664 \quad 23.485$

$24.427 \quad 24.242$

23.308

$2 / .060$

24.812

25.191

25.000

$25.954 \quad 25.753$

25.564

2.6 .316

1.193
2.239
2.985
$3.73 ?$

0.747

1.481

2.222

2.963

3.704

4.478

5.224

5.970

6.716

7.463

$4.1,14$

5.185

5.926

6.667

7.407

8.209

8.955

9.701

$10.1,1,8$

8.148

8.889

9.630

10.370

11.194

11.111

\subsection{0 \\ 11.852}

12.687

12.593

13.1433

13.333

14.179

14.074

14.925

14.815

\subsection{2 \\ 15.556}

16.418

17.164

17.910

16.296

17.037

17.778

18.557

18.519

19.103

20.149

20.896

21.642

22.388

19.259

20.000

20.7 : 1

21.481

22.272

23.134

23.881

24.627

25.373

26.119

22.963

23.704

24.444

25.185

25.926

136

137

138

139.

$1 / 0$

0.735
1.471
2.206
2.941
3.676

0.730

0.725

1.460

2.190

2.920

3.650

1.1449

2.174

2.899

3.623

0.719

0.714

4.412

5.147

5.882

6.618

7.353

4.380

5.109

5.839

6.569

7.299

4.348

5.072

5.797

6.522

7.246

1.439

2.158

2.878

3.597

1.429

2.143

2.857

8.088

8.824

9.559

10.294

8.029

8.759

7.971

9.489

8.696

10.219

9.420

11.020

10.949

10.145

10.870

4.317

5.036

5.755

6.475

7.194

3.571

$\begin{array}{ll}11.765 & 11.679\end{array}$

12.500

11.594

12.319

13.235

13.139

$13.971 \quad 13.869$

$14.706 \quad 14.599$

13.043

13.768

14.493

$15.441 \quad 15.328$

16.176

16.912

17.647

16.058

15.217

15.942

16.788

17.515
18.248

16.667

17.391

18.116

19.116

19.853

18.978

20.588

21.324

19.708

18.847

20.438

22.059

21.168

21.898

19.565

20.290

21.014

21.739

7.914

8.633

9.353

10.072

10.791

4.286

5.000

5.714

6.429

7.143

22.794

23.529

22.628

22.464

24.265

23.358

24.088

23.188

23.913

24.638 



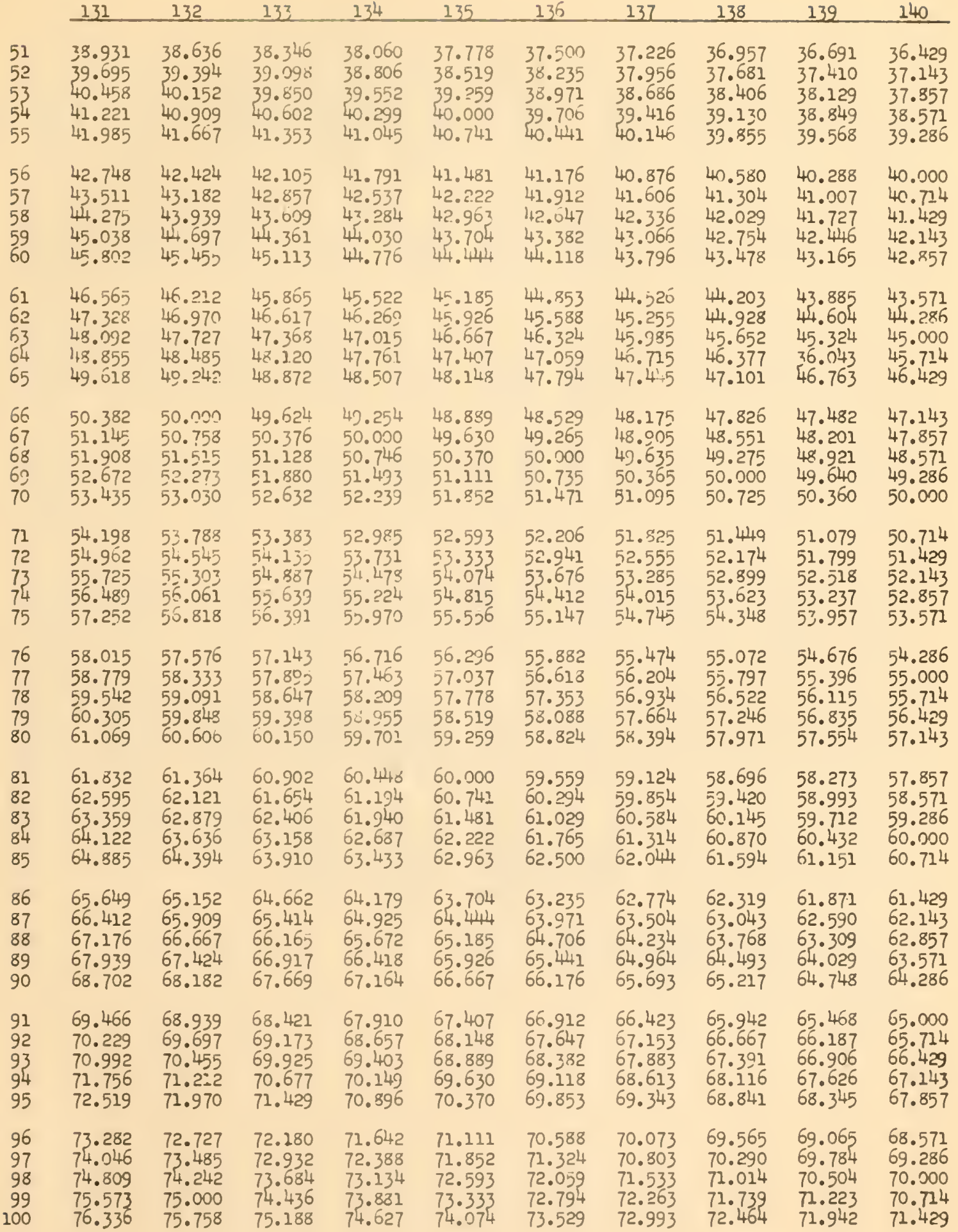





\begin{tabular}{|c|c|c|c|c|c|c|c|c|c|c|}
\hline & 131 & 132 & 133 & 134 & 135 & 136 & 137 & 138 & 139 & 140 \\
\hline & $\begin{array}{l}77.099 \\
77.863 \\
78.626 \\
79.389 \\
30.153\end{array}$ & $\begin{array}{l}76.515 \\
77.273 \\
78.030 \\
78.788 \\
79.545\end{array}$ & & & & & $\begin{array}{l}73.723 \\
74.453 \\
75.182 \\
75.912 \\
76.642\end{array}$ & & & $\begin{array}{l}72.143 \\
72.857 \\
73.571 \\
74.286 \\
75.000\end{array}$ \\
\hline & $\begin{array}{l}80.916 \\
81.679 \\
82.443 \\
83.206 \\
83.969\end{array}$ & & $\begin{array}{l}79.699 \\
80.451 \\
81.203 \\
81.955 \\
82.707\end{array}$ & & & & & & & $\begin{array}{l}75.714 \\
76.429 \\
77.143 \\
77.857 \\
78.571\end{array}$ \\
\hline & $\begin{array}{l}84.733 \\
85.496 \\
86.260 \\
87.023 \\
87.780\end{array}$ & & $\begin{array}{l}83.459 \\
84.211 \\
84.962 \\
85.714 \\
80.406\end{array}$ & $\begin{array}{l}82.836 \\
83.582 \\
84.328 \\
85.075 \\
85.821\end{array}$ & $\begin{array}{l}82.222 \\
32.963 \\
83.704 \\
84.444 \\
85.185\end{array}$ & & & & $\begin{array}{l}79.856 \\
80.576 \\
81.295 \\
82.014 \\
82.734\end{array}$ & $\begin{array}{l}79.286 \\
80.200 \\
80.714 \\
81.429 \\
82.143\end{array}$ \\
\hline & $\begin{array}{l}88.550 \\
89.313 \\
90.076 \\
90.840 \\
91.603\end{array}$ & & $\begin{array}{l}87.218 \\
87.970 \\
88.722 \\
89.474 \\
90.226\end{array}$ & & & & & & & $\begin{array}{l}82.857 \\
83.571 \\
84.286 \\
85.000 \\
85.714\end{array}$ \\
\hline & $\begin{array}{l}92.366 \\
93.130 \\
93.393 \\
94.656 \\
95.420\end{array}$ & $\begin{array}{l}91.667 \\
92.424 \\
93.182 \\
93.939 \\
94.697\end{array}$ & & & & & & & & $\begin{array}{l}86.429 \\
87.143 \\
87.857 \\
88.571 \\
89.286\end{array}$ \\
\hline & $\begin{array}{l}96.183 \\
96.947 \\
97.710 \\
98.473 \\
99.237\end{array}$ & $\begin{array}{l}95.455 \\
05.212 \\
96.970 \\
97.727 \\
98.485\end{array}$ & $\begin{array}{l}94.737 \\
95.489 \\
96.241 \\
96.992 \\
97.744\end{array}$ & $\begin{array}{l}94.030 \\
94.776 \\
95.522 \\
96.269 \\
97.015\end{array}$ & $\begin{array}{l}93.333 \\
94.074 \\
94.815 \\
95.556 \\
96.296\end{array}$ & & $\begin{array}{l}91.971 \\
92.701 \\
93.431 \\
94.161 \\
94.891\end{array}$ & & & $\begin{array}{l}90.200 \\
90.714 \\
91.429 \\
92.143 \\
92.857\end{array}$ \\
\hline & $\begin{array}{l}00.000 \\
--- \\
--- \\
---\end{array}$ & $\begin{array}{c}99.242 \\
100.000 \\
--- \\
--\end{array}$ & $\begin{array}{c}98.496 \\
99.248 \\
100.000 \\
--- \\
---\end{array}$ & $\begin{array}{r}37.761 \\
98.507 \\
99.254 \\
100.000 \\
\end{array}$ & $\begin{array}{r}97.037 \\
17.778 \\
98.519 \\
99.259 \\
100.000\end{array}$ & & & & & $\begin{array}{l}93.571 \\
94.286 \\
95.000 \\
95.714 \\
96.429\end{array}$ \\
\hline & $\begin{array}{l}-- \\
-- \\
--- \\
--\end{array}$ & $\begin{array}{l}--- \\
--- \\
--- \\
--\end{array}$ & $\begin{array}{l}--- \\
--- \\
--- \\
---\end{array}$ & $\begin{array}{l}--- \\
-- \\
--- \\
--\end{array}$ & $\begin{array}{l}--- \\
--- \\
--- \\
---\end{array}$ & $\begin{array}{c}100.000 \\
-- \\
-- \\
--\end{array}$ & $\begin{array}{c}99.270 \\
100.200 \\
--- \\
---\end{array}$ & $\begin{array}{r}98.551 \\
99.275 \\
100.000 \\
--- \\
---\end{array}$ & $\begin{array}{r}97.842 \\
98.561 \\
99.281 \\
100.000 \\
-.-\end{array}$ & $\begin{array}{r}97.143 \\
97.357 \\
93.571 \\
99.286 \\
100.000\end{array}$ \\
\hline
\end{tabular}





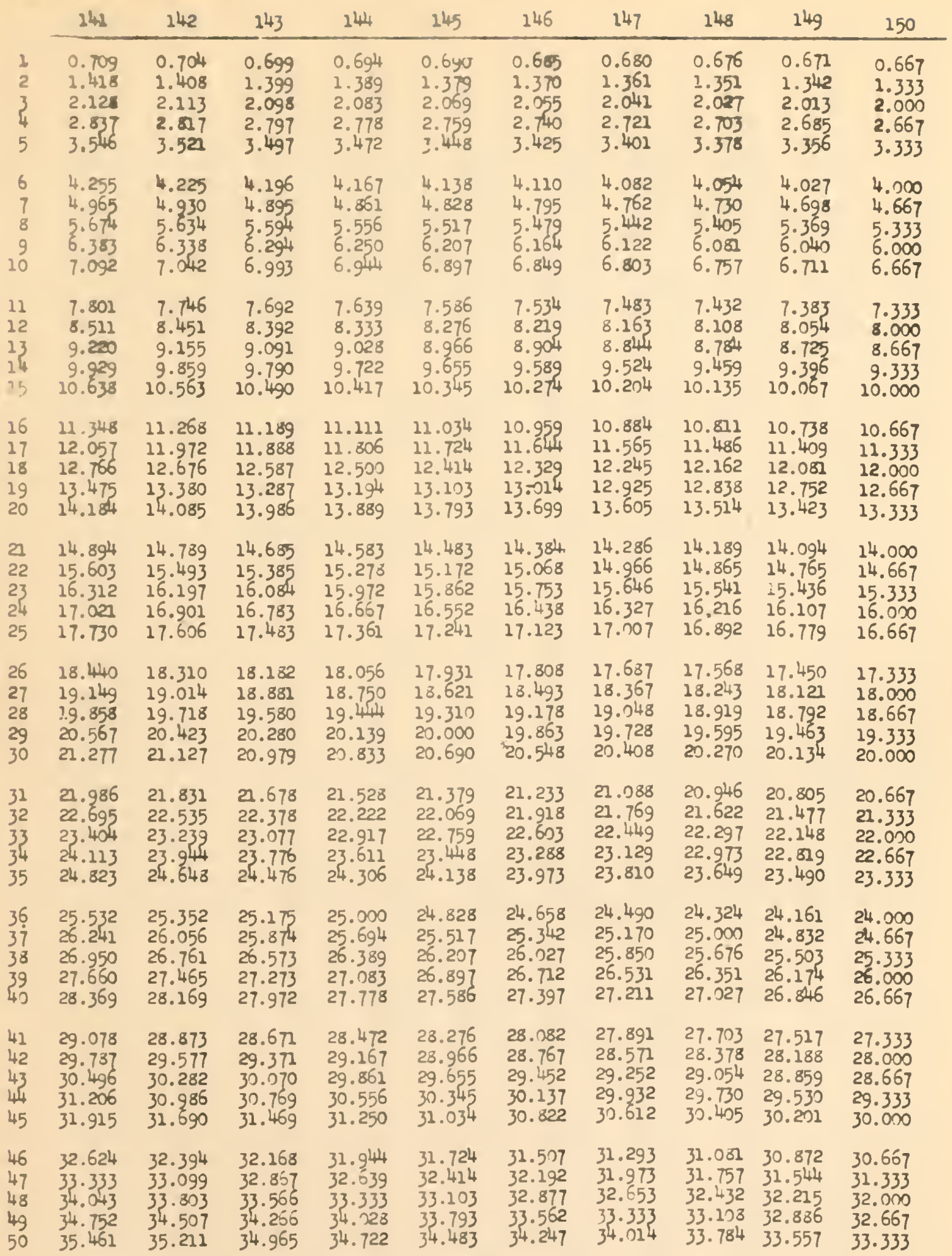





\begin{tabular}{|c|c|c|c|c|c|c|c|c|c|}
\hline 141 & 142 & 143 & 144 & 145 & 146 & 147 & 148 & 149 & 150 \\
\hline $\begin{array}{l}36.170 \\
36.879\end{array}$ & $\begin{array}{l}35.915 \\
36.620 \\
37.324 \\
38.028 \\
38.732\end{array}$ & $\begin{array}{l}35.664 \\
36.364 \\
37.063 \\
37.762 \\
38.462\end{array}$ & $\begin{array}{l}35.417 \\
36.111 \\
36.806 \\
37.500 \\
38.194\end{array}$ & $\begin{array}{l}35.172 \\
35.862 \\
36.552 \\
37.241 \\
37.931\end{array}$ & $\begin{array}{l}34.932 \\
35.616 \\
36.301 \\
36.986 \\
37.671\end{array}$ & & & & $\begin{array}{l}34.000 \\
34.667 \\
35.333 \\
36.000 \\
36.667\end{array}$ \\
\hline $\begin{array}{l}39.716 \\
40.426 \\
41.135 \\
41.844 \\
42.553\end{array}$ & $\begin{array}{l}39.437 \\
40.141 \\
40.845 \\
41.549 \\
42.254\end{array}$ & $\begin{array}{l}39.161 \\
39.860 \\
40.559\end{array}$ & $\begin{array}{l}38.889 \\
39.583 \\
40.278\end{array}$ & $\begin{array}{l}38.621 \\
39.310 \\
40.000 \\
40.690 \\
41.379\end{array}$ & $\begin{array}{l}38.356 \\
39.041 \\
39.726 \\
40.411 \\
41.096\end{array}$ & & & & $\begin{array}{l}37.333 \\
38.000 \\
38.667 \\
39.333 \\
40.000\end{array}$ \\
\hline $\begin{array}{l}43.262 \\
43.972 \\
44.681 \\
45.390 \\
46.099\end{array}$ & $\begin{array}{l}42.958 \\
43.662 \\
44.366 \\
45.070 \\
45.775\end{array}$ & $\begin{array}{l}42.657 \\
43.357 \\
44.056 \\
44.755 \\
45.455\end{array}$ & $\begin{array}{l}42.361 \\
43.056 \\
43.750 \\
44.444 \\
45.139\end{array}$ & $\begin{array}{l}42.069 \\
42.759 \\
43.448 \\
44.138 \\
44.828\end{array}$ & $\begin{array}{l}41.781 \\
42.466 \\
43.151 \\
43.836 \\
44.521\end{array}$ & & & & $\begin{array}{l}40.667 \\
41.333 \\
42.000 \\
42.667 \\
43.333\end{array}$ \\
\hline $\begin{array}{l}46.809 \\
47.518 \\
48.227 \\
48.936 \\
49.645\end{array}$ & & & $\begin{array}{l}45.833 \\
46.528 \\
47.222 \\
47.917 \\
48.611\end{array}$ & & $\begin{array}{l}45.205 \\
45.890 \\
46.575 \\
47.260 \\
47.945\end{array}$ & & & $\begin{array}{l}44.295 \\
44.966 \\
45.638 \\
46.309 \\
46.980\end{array}$ & $\begin{array}{l}44.000 \\
44.667 \\
45.333 \\
46.000 \\
46.667\end{array}$ \\
\hline $\begin{array}{l}50.355 \\
51.064 \\
51.773 \\
52.482 \\
53.191\end{array}$ & $\begin{array}{l}50.000 \\
50.704 \\
51.408 \\
52.113 \\
52.817\end{array}$ & $\begin{array}{l}49.650 \\
50.350 \\
51.049 \\
51.748 \\
52.448\end{array}$ & $\begin{array}{l}49.306 \\
50.000 \\
50.694 \\
51.389 \\
52.083\end{array}$ & & $\begin{array}{l}48.630 \\
49.315 \\
50.000 \\
50.685 \\
51.370\end{array}$ & & & & $\begin{array}{l}+7.333 \\
48.000 \\
48.667 \\
49.333 \\
50.000\end{array}$ \\
\hline $\begin{array}{l}53.901 \\
54.620 \\
55.319 \\
56.028 \\
56.738\end{array}$ & $\begin{array}{l}53.521 \\
54.225 \\
54.930 \\
55.634 \\
56.338\end{array}$ & $\begin{array}{l}53.147 \\
53.846 \\
54.545 \\
55.245 \\
55.944\end{array}$ & $\begin{array}{l}52.778 \\
53.472 \\
54.167 \\
54.861 \\
55.556\end{array}$ & $\begin{array}{l}52.414 \\
53.103 \\
53.793 \\
54.483 \\
55.172\end{array}$ & $\begin{array}{l}52.055 \\
52.740 \\
53.425 \\
54.110 \\
54.795\end{array}$ & $\begin{array}{l}53.741 \\
54.422\end{array}$ & & & $\begin{array}{l}50.667 \\
51.333 \\
52.000 \\
52.667 \\
53.333\end{array}$ \\
\hline $\begin{array}{l}57.447 \\
58.156 \\
58.865 \\
59.574 \\
60.284\end{array}$ & $\begin{array}{l}57.042 \\
57.746 \\
58.451 \\
59.155 \\
59.859\end{array}$ & $\begin{array}{l}56.643 \\
57.343 \\
58.042 \\
58.741 \\
59.441\end{array}$ & $\begin{array}{l}56.250 \\
56.0 .44 \\
57.639 \\
58.333 \\
59.028\end{array}$ & & $\begin{array}{l}55.479 \\
56.164 \\
56.849 \\
57.534 \\
58.219\end{array}$ & $\begin{array}{l}55.102 \\
55.782 \\
56.463 \\
57.143 \\
57.823\end{array}$ & & $\begin{array}{l}54.362 \\
55.034 \\
55.705 \\
56.376 \\
57.047\end{array}$ & $\begin{array}{l}54.000 \\
54.667 \\
55.333 \\
56.000 \\
56.667\end{array}$ \\
\hline $\begin{array}{l}60.993 \\
61.702 \\
62.411 \\
63.121 \\
63.830\end{array}$ & $\begin{array}{l}60.563 \\
61.268 \\
61.972 \\
62.676 \\
63.380\end{array}$ & $\begin{array}{l}60.140 \\
60.839 \\
61.538 \\
52.238 \\
62.937\end{array}$ & & & $\begin{array}{l}58.904 \\
59.589 \\
60.274 \\
60.959 \\
61.644\end{array}$ & $\begin{array}{l}58.503 \\
59.184 \\
59.864 \\
60.544\end{array}$ & & & $\begin{array}{l}57.333 \\
58.000 \\
58.667 \\
59.333 \\
60.000\end{array}$ \\
\hline $\begin{array}{l}64.539 \\
65.248 \\
65.057 \\
66.667 \\
67.376\end{array}$ & $\begin{array}{l}64.085 \\
64.769 \\
65.493 \\
66.197 \\
66.901\end{array}$ & $\begin{array}{l}63.636 \\
64.336 \\
65.035 \\
65.734 \\
66.434\end{array}$ & $\begin{array}{l}63.194 \\
63.889 \\
64.583 \\
65.278 \\
65.972\end{array}$ & $\begin{array}{l}62.759 \\
63.448 \\
64.138 \\
64.828 \\
65.517\end{array}$ & $\begin{array}{l}62.329 \\
63.014 \\
63.699 \\
64.384 \\
65.068\end{array}$ & $\begin{array}{l}61.905 \\
62.585 \\
63.265 \\
63.946 \\
64.626\end{array}$ & & & $\begin{array}{l}60.667 \\
61.333 \\
62.000 \\
62.667 \\
63.333\end{array}$ \\
\hline $\begin{array}{l}085 \\
794 \\
504 \\
213\end{array}$ & $\begin{array}{l}67.606 \\
68.310 \\
69.014 \\
69.718 \\
70.423\end{array}$ & $\begin{array}{l}67.832 \\
68.531 \\
69.231 \\
69.930\end{array}$ & $\begin{array}{l}66.667 \\
67.361 \\
68.056 \\
68.750 \\
69.444\end{array}$ & $\begin{array}{l}66.207 \\
66.897 \\
67.586 \\
68.276 \\
68.066\end{array}$ & $\begin{array}{l}65.753 \\
66.438 \\
67.123 \\
67.808 \\
68.493\end{array}$ & $\begin{array}{l}65.306 \\
65.086 \\
66.667 \\
67.347 \\
68.027\end{array}$ & $\begin{array}{l}64.865 \\
65.541 \\
66.216 \\
66.892 \\
67.568\end{array}$ & $\begin{array}{l}64.430 \\
65.101 \\
65.772 \\
66.443 \\
67.114\end{array}$ & $\begin{array}{l}64.000 \\
64.667 \\
65.333 \\
66.000 \\
66.667\end{array}$ \\
\hline
\end{tabular}





\begin{tabular}{|c|c|c|c|c|c|c|c|c|c|c|}
\hline & 141 & 142 & 143 & 144 & 145 & 146 & 147 & 148 & 149 & 150 \\
\hline & 74.468 & & $\begin{array}{l}72 . \\
72 . \\
73 .\end{array}$ & & & & & & $\begin{array}{l}67.785 \\
68.456 \\
69.128 \\
69.799 \\
70.470\end{array}$ & $\begin{array}{l}67.333 \\
68.000 \\
68.667 \\
69.333 \\
70.000\end{array}$ \\
\hline & $\begin{array}{l}76.596 \\
77.305 \\
78.014\end{array}$ & $\begin{array}{l}74.648 \\
75.352 \\
76.056 \\
76.761 \\
77.465\end{array}$ & $\begin{array}{l}74.126 \\
74.825 \\
75.524 \\
76.224 \\
76.923\end{array}$ & & $\begin{array}{l}73.103 \\
73.793 \\
74.483 \\
75.172 \\
75.862\end{array}$ & $\begin{array}{l}72.603 \\
73.288 \\
73.973 \\
74.658 \\
75.342\end{array}$ & & & & $\begin{array}{l}70.667 \\
71.333 \\
72.000 \\
72.667 \\
73.333\end{array}$ \\
\hline & $\begin{array}{l}78.723 \\
79.433 \\
80.142 \\
80.851 \\
81.560\end{array}$ & $\begin{array}{l}78.169 \\
78.873 \\
79.577 \\
80.282 \\
80.986\end{array}$ & & & & & & & $\begin{array}{l}74.497 \\
75.168 \\
75.839 \\
76.510 \\
77.181\end{array}$ & $\begin{array}{l}74.000 \\
74.667 \\
75.333 \\
76.000 \\
76.667\end{array}$ \\
\hline & $\begin{array}{l}82.270 \\
82.979 \\
83.688 \\
84.397 \\
85.106\end{array}$ & & & & & & & & & $\begin{array}{l}77.333 \\
78.000 \\
78.667 \\
79.333 \\
80.000\end{array}$ \\
\hline 125 & $\begin{array}{l}85.816 \\
86.525 \\
87.234 \\
87.943 \\
88.652\end{array}$ & $\begin{array}{l}85.211 \\
85.915 \\
86.620 \\
87.324 \\
88.028\end{array}$ & $\begin{array}{l}84.615 \\
85.315 \\
86.014 \\
86.713 \\
87.413\end{array}$ & $\begin{array}{l}84.028 \\
84.722 \\
85.417 \\
86.111 \\
86.806\end{array}$ & & & & & & $\begin{array}{l}80.667 \\
81.333 \\
82.000 \\
82.667 \\
83.333\end{array}$ \\
\hline & & & & & & & & & & $\begin{array}{l}84.000 \\
84.667 \\
85.333 \\
86.000 \\
86.667\end{array}$ \\
\hline 130 & $\begin{array}{l}92.908 \\
93.617 \\
94.326 \\
95.035 \\
95.745\end{array}$ & $\begin{array}{l}92.254 \\
92.958 \\
93.662 \\
94.366 \\
95.070\end{array}$ & & & & & & & & \\
\hline & $\begin{array}{l}96.454 \\
97.163 \\
97.872 \\
98.582 \\
99.291\end{array}$ & $\begin{array}{l}95.775 \\
96.479 \\
97.183 \\
97.887 \\
98.592\end{array}$ & $\begin{array}{l}95.105 \\
95.804 \\
96.503 \\
97.203 \\
97.902\end{array}$ & & & & & & & $\begin{array}{l}90.667 \\
91.333 \\
92.000 \\
92.667 \\
93.333\end{array}$ \\
\hline & 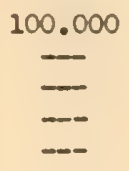 & $\begin{array}{c}99.296 \\
100.000 \\
- \\
-\end{array}$ & $\begin{array}{r}98.601 \\
99.301 \\
100.000 \\
- \\
\end{array}$ & $\begin{array}{r}97.917 \\
98.611 \\
99.306 \\
100.000 \\
\end{array}$ & $\begin{array}{r}97.241 \\
97.931 \\
98.621 \\
99.310 \\
100.000\end{array}$ & & & & $\begin{array}{l}94.361 \\
95.302 \\
95.973 \\
96.644 \\
97.315\end{array}$ & $\begin{array}{l}94.000 \\
94.667 \\
95.333 \\
96.000 \\
96.667\end{array}$ \\
\hline & 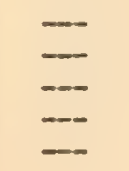 & $=$ & $\overline{-}$ & $\overline{--}$ & $\begin{array}{l}--- \\
--- \\
-- \\
--\end{array}$ & ${ }^{100.000}=$ & $\begin{array}{c}99.320 \\
100.000 \\
\ldots \\
=\end{array}$ & $\begin{array}{r}98.649 \\
99.324 \\
100.000 \\
\ldots \\
\ldots-\end{array}$ & $\begin{array}{r}97.987 \\
98.658 \\
99.329 \\
100.000 \\
\ldots\end{array}$ & $\begin{array}{r}97.333 \\
98.000 \\
98.667 \\
99.333 \\
100.000\end{array}$ \\
\hline
\end{tabular}




\begin{tabular}{|c|c|c|c|c|c|c|c|c|c|c|}
\hline & 151 & 152 & 153 & 154 & 155 & 156 & 157 & 158 & 159 & 160 \\
\hline $\begin{array}{l}1 \\
2 \\
3 \\
4 \\
5\end{array}$ & $\begin{array}{l}0.662 \\
1.325 \\
1.987 \\
2.649 \\
3.311\end{array}$ & $\begin{array}{l}0.658 \\
1.316 \\
1.974 \\
2.532 \\
3.289\end{array}$ & $\begin{array}{l}0.654 \\
1.307 \\
1.961 \\
2.614 \\
3.268\end{array}$ & $\begin{array}{l}0.649 \\
1.299 \\
1.948 \\
2.597 \\
3.247\end{array}$ & $\begin{array}{l}0.645 \\
1.290 \\
1.935 \\
2.581 \\
3.226\end{array}$ & $\begin{array}{l}0.641 \\
1.282 \\
1.923 \\
2.564 \\
3.205\end{array}$ & $\begin{array}{l}0.637 \\
1.274 \\
1.911 \\
2.548 \\
3.185\end{array}$ & $\begin{array}{l}0.633 \\
1.266 \\
1.899 \\
2.532 \\
3.165\end{array}$ & $\begin{array}{l}0.629 \\
1.258 \\
1.887 \\
2.516 \\
3.145\end{array}$ & $\begin{array}{l}0.625 \\
1.250 \\
1.875 \\
2.500 \\
3.125\end{array}$ \\
\hline $\begin{array}{l}6 \\
7 \\
8 \\
9\end{array}$ & $\begin{array}{l}3.974 \\
4.636 \\
5.298 \\
5.960 \\
6.623\end{array}$ & $\begin{array}{l}3.947 \\
4.605 \\
5.263 \\
5.921 \\
6.579\end{array}$ & $\begin{array}{l}3.922 \\
4.575 \\
5.229 \\
5.882 \\
6.536\end{array}$ & $\begin{array}{l}3.896 \\
4.545 \\
5.195 \\
5.844 \\
6.494\end{array}$ & $\begin{array}{l}3.871 \\
4.516 \\
5.161 \\
5.806 \\
6.452\end{array}$ & $\begin{array}{l}3.846 \\
4.487 \\
5.128 \\
5.769 \\
6.410\end{array}$ & $\begin{array}{l}3.822 \\
4.459 \\
5.096 \\
5.732 \\
6.359\end{array}$ & $\begin{array}{l}3.797 \\
4.430 \\
5.063 \\
5.696 \\
6.329\end{array}$ & $\begin{array}{l}3.774 \\
4.403 \\
5.031 \\
5.660 \\
6.289\end{array}$ & $\begin{array}{l}3.750 \\
4.375 \\
5.000 \\
5.525 \\
6.250\end{array}$ \\
\hline & $\begin{array}{l}7.285 \\
7.947 \\
8.609 \\
9.272 \\
9.934\end{array}$ & $\begin{array}{l}7.237 \\
7.895 \\
8.553 \\
9.211 \\
9.868\end{array}$ & $\begin{array}{l}7.190 \\
7.843 \\
8.497 \\
9.150 \\
9.804\end{array}$ & $\begin{array}{l}7.143 \\
7.792 \\
8.442 \\
9.091 \\
9.740\end{array}$ & $\begin{array}{l}7.097 \\
7.742 \\
8.387 \\
9.032 \\
9.677\end{array}$ & $\begin{array}{l}7.051 \\
7.692 \\
8.333 \\
8.974 \\
9.615\end{array}$ & $\begin{array}{l}7.006 \\
7.643 \\
8.280 \\
8.917 \\
3.554\end{array}$ & $\begin{array}{l}6.962 \\
7.595 \\
8.228 \\
8.861 \\
9.494\end{array}$ & $\begin{array}{l}6.918 \\
7.547 \\
8.276 \\
8.805 \\
9.434\end{array}$ & $\begin{array}{l}6.875 \\
7.500 \\
8.125 \\
8.750 \\
9.375\end{array}$ \\
\hline & $\begin{array}{l}10.596 \\
11.258 \\
11.921 \\
12.583 \\
13.245\end{array}$ & $\begin{array}{l}10.526 \\
11.184 \\
11.842 \\
12.500 \\
13.158\end{array}$ & $\begin{array}{l}10.458 \\
11.111 \\
11.765 \\
12.418 \\
13.072\end{array}$ & $\begin{array}{l}10.390 \\
11.039 \\
11.688 \\
12.338 \\
12.987\end{array}$ & & $\begin{array}{l}10.256 \\
10.897 \\
11.538 \\
12.179 \\
12.821\end{array}$ & $\begin{array}{l}10.191 \\
10.828 \\
11.465 \\
12.102 \\
12.739\end{array}$ & & $\begin{array}{l}10.063 \\
10.692 \\
11.321 \\
11.950 \\
12.579\end{array}$ & $\begin{array}{l}10.000 \\
10.625 \\
11.250 \\
11.875 \\
12.500\end{array}$ \\
\hline & $\begin{array}{l}13.907 \\
14.570 \\
15.232 \\
15.894 \\
16.556\end{array}$ & $\begin{array}{l}13.816 \\
14.474 \\
15.132 \\
15.789 \\
16.447\end{array}$ & $\begin{array}{l}13.725 \\
14.379 \\
15.033 \\
15.636 \\
16.340\end{array}$ & $\begin{array}{l}13.636 \\
14.236 \\
14.935 \\
15.584 \\
16.234\end{array}$ & $\begin{array}{l}13.548 \\
14.194 \\
14.339 \\
15.484 \\
16.129\end{array}$ & $\begin{array}{l}13.462 \\
14.103 \\
14.744 \\
15.335 \\
16.026\end{array}$ & $\begin{array}{l}13.376 \\
14.013 \\
14.650 \\
15.287 \\
15.924\end{array}$ & $\begin{array}{l}13.291 \\
13.924 \\
14.557 \\
15.190 \\
15.823\end{array}$ & $\begin{array}{l}13.208 \\
13.836 \\
14.465 \\
15.094 \\
15.723\end{array}$ & $\begin{array}{l}13.125 \\
13.750 \\
14.375 \\
15.000 \\
15.625\end{array}$ \\
\hline & $\begin{array}{l}17.219 \\
17.381 \\
18.543 \\
19.295 \\
19.868\end{array}$ & $\begin{array}{l}17.105 \\
17.763 \\
18.421 \\
19.079 \\
19.737\end{array}$ & $\begin{array}{l}16.993 \\
17.647 \\
18.301 \\
18.954 \\
19.608\end{array}$ & $\begin{array}{l}16.883 \\
17.532 \\
18.182 \\
18.831 \\
19.481\end{array}$ & & $\begin{array}{l}16.567 \\
17.308 \\
17.949 \\
18.590 \\
19.231\end{array}$ & & & $\begin{array}{l}16.352 \\
16.981 \\
17.610 \\
18.239 \\
18.868\end{array}$ & $\begin{array}{l}16.250 \\
16.875 \\
17.500 \\
18.125 \\
18.750\end{array}$ \\
\hline 4 & $\begin{array}{l}20.530 \\
21.192 \\
21.354 \\
22.517 \\
23.179\end{array}$ & $\begin{array}{l}20.395 \\
21.053 \\
21.711 \\
22.368 \\
23.026\end{array}$ & $\begin{array}{l}20.261 \\
20.915 \\
21.559 \\
22.222 \\
22.576\end{array}$ & $\begin{array}{l}20.130 \\
20.779 \\
21.429 \\
22.778 \\
22.727\end{array}$ & & $\begin{array}{l}19.372 \\
20.513 \\
21.154 \\
21.795 \\
22.436\end{array}$ & $\begin{array}{l}19.745 \\
20.382 \\
21.019 \\
21.656 \\
22.293\end{array}$ & & $\begin{array}{l}19.497 \\
20.126 \\
20.755 \\
21.384 \\
22.013\end{array}$ & $\begin{array}{l}19.375 \\
20.000 \\
20.625 \\
21.250 \\
21.875\end{array}$ \\
\hline $\begin{array}{l}35 \\
37 \\
3 z \\
39 \\
40\end{array}$ & $\begin{array}{l}23.341 \\
24.503 \\
25.106 \\
25.328 \\
26.490\end{array}$ & $\begin{array}{l}23.684 \\
24.342 \\
25.000 \\
25.650 \\
26.316\end{array}$ & $\begin{array}{l}23.529 \\
24.183 \\
24.637 \\
25.490 \\
26.144\end{array}$ & $\begin{array}{l}23.377 \\
24.226 \\
24.675 \\
25.325 \\
25.974\end{array}$ & & $\begin{array}{l}23.077 \\
23.718 \\
24.359 \\
25.020 \\
25.641\end{array}$ & $\begin{array}{l}22.930 \\
23.567 \\
24.204 \\
24.841 \\
25.478\end{array}$ & $\begin{array}{l}22.785 \\
23.418 \\
24.051 \\
24.684 \\
25.316\end{array}$ & $\begin{array}{l}22.642 \\
23.270 \\
23.899 \\
24.528 \\
25.157\end{array}$ & $\begin{array}{l}22.500 \\
23.125 \\
23.750 \\
24.375 \\
25.000\end{array}$ \\
\hline 3 & $\begin{array}{l}27.152 \\
27.015 \\
28.477 \\
29.139 \\
29.801\end{array}$ & $\begin{array}{l}26.974 \\
27.632 \\
28.269 \\
28.947 \\
29.605\end{array}$ & $\begin{array}{l}26.797 \\
27.451 \\
23.105 \\
28.758 \\
29.412\end{array}$ & $\begin{array}{l}26.623 \\
27.273 \\
27.922 \\
28.571 \\
29.221\end{array}$ & $\begin{array}{l}26.452 \\
27.097 \\
27.742 \\
28.387 \\
29.032\end{array}$ & $\begin{array}{l}26.232 \\
20.923 \\
27.564 \\
28.205 \\
28.846\end{array}$ & $\begin{array}{l}26.115 \\
26.752 \\
27.389 \\
28.725 \\
28.662\end{array}$ & $\begin{array}{l}25.949 \\
26.582 \\
27.215 \\
27.348 \\
28.431\end{array}$ & $\begin{array}{l}25.786 \\
26.415 \\
27.044 \\
27.673 \\
28.302\end{array}$ & $\begin{array}{l}25.625 \\
26.250 \\
26.875 \\
27.500 \\
28.125\end{array}$ \\
\hline 9 & $\begin{array}{l}30.404 \\
31.126 \\
31.738 \\
32.450 \\
33.113\end{array}$ & $\begin{array}{l}30.263 \\
30.721 \\
31.579 \\
32.237 \\
32.895\end{array}$ & $\begin{array}{l}30.065 \\
30.719 \\
31.373 \\
32.026 \\
32.680\end{array}$ & $\begin{array}{l}29.370 \\
30.519 \\
31.169 \\
31.818 \\
32.468\end{array}$ & $\begin{array}{l}29.677 \\
30.323 \\
30.968 \\
31.613 \\
32.258\end{array}$ & $\begin{array}{l}29.487 \\
30.128 \\
30.769 \\
31.410 \\
32.051\end{array}$ & $\begin{array}{l}29.299 \\
29.936 \\
30.573 \\
31.210 \\
31.847\end{array}$ & $\begin{array}{l}29.114 \\
29.747 \\
30.380 \\
31.013 \\
31.046\end{array}$ & $\begin{array}{l}28.931 \\
29.560 \\
30.189 \\
30.818 \\
31.447\end{array}$ & $\begin{array}{l}28.750 \\
29.375 \\
30.000 \\
30.625 \\
31.250\end{array}$ \\
\hline
\end{tabular}





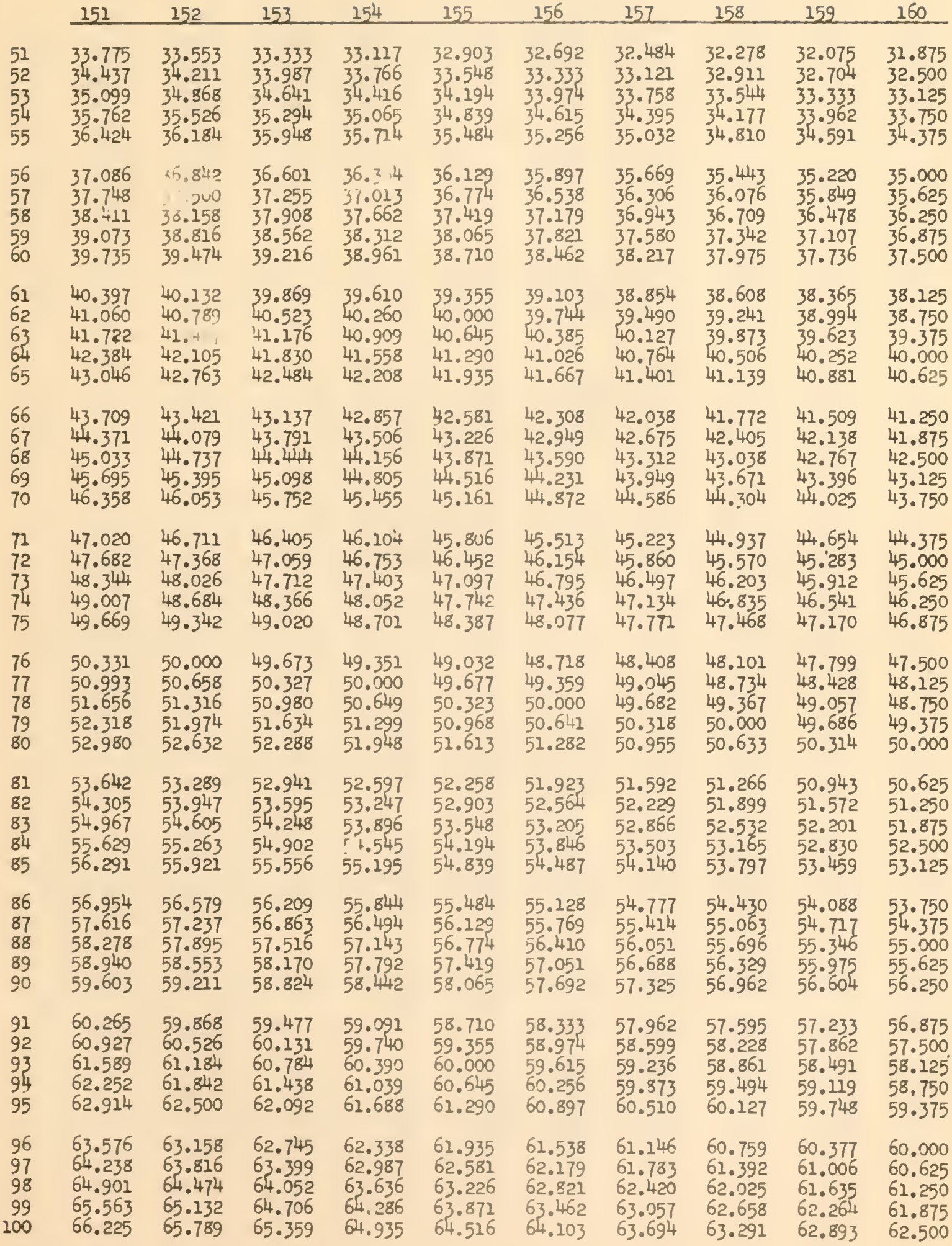


151

153

154

155

156

157

158

159

160

101

$66.887 \quad 66.447 \quad 66.013$

102

103

104

105

106

107

108

110

111

112

11

115

11676.82

$117 \quad 77.483$

11878.146

11978.808

$120 \quad 79.470$

12

122

12

12

125

126

127

128

129

130

13186.755

13287.417

13388.079

13488.742

13589.404

13690.066

13790.728

13891.391

13992.053

$140 \quad 92.715$

14183.377

14294.040

14394.702

14495.364

14596.026

146

147

148

149

150

\subsection{7}

67.320

67.763

68.421

67.974

69.079

68.627

\subsection{7}

69.281

69.935

70.588

71.053

71.711

72.368

71.242

71.895

73.026

73.684

74.342

75.000

75.658

72.549

73.856

74.510

75.163

76.316

76.974

77.632

75.817

76.471

77.124

78.289

78.947

78.431

79.605

80.263

80.921

81.579

79.085

79.739

80.392

81.046

81.699

82.353

83.007

83.660

83.553

84.211

84.868

84.314

86.184

86.842

87.500

88.158

88.816

89.474

90.132

90.789

91.447

92.105

85.621

86.275

86.928

87.582

88.235

88.889

89.543

90.196

90.850

91.503

92.763

93.421

92.157

92.810

94.079

94.737

93.464

94.118

95.395

94.771

95.425

96.68996 .053

97.351

96.711

96.078

97.368

98.67598 .026

99.338
95.684
73.203

77.778

65.584

66.234

66.883

67.532

68.182

65.161

65.806

66.452

67.097

68.831

69.481

$7 C .130$

70.779

71.429

67.742

72.078

72.727

73.377

74.026

74.675

68.387

69.032

69.677

70.323

70.968

75.325

75.974

$7 \in .623$

71.613

72.258

72.903

73.548

74.194

77.273

77.922

74.839

75.484

76.129

76.774

77.419

78.571

79.221

78.065

79.870

78.710

79.355

80.519

81.169

80.000

80.645

81.818

81. 290

82.468

83.117

83.766

81.935

82.581

83.226

83.871

85.065

85.714

86.364

87.013

87.662

84.516

85.161

85.806

86.452

87.097

88.312

88.961

89.610

87.742

88.387

89.032

90.260

90.909

89.677

90.323

91.558

90.968

82.208

92.857

91.613

92.258

93.506

92.903

93.548

94.156

94.194

94.805
96.732

97.386

95.455

96.104

96.753

94.839

95.484

96.129

96.774

64.744

65.385

66.026

66.667

67.308

67.949

68.590

69.231

69.372

70.513

71.151

71.795

72.436

73.077

73.718

74.359

75.000

75.641

76.282

76.923

77.564

78.205

78.846

79.487

80.128

80.769

81.410

82.051

82.692

83.333

64.331

64.968

65.605

66.242

66.879 

P IRCMANTAGI TABLES

$\begin{array}{rrrrrrrrrrr} & 151 & 152 & 153 & 154 & 155 & 156 & 157 & 158 & 159 & 160 \\ 151 & 100.000 & 99.342 & 98.693 & 98.052 & 97.419 & 96.795 & 96.178 & 95.570 & 94.969 & 94.375 \\ 152 & - & 100.000 & 99.346 & 98.701 & 98.065 & 97.436 & 96.815 & 96.203 & 95.597 & 95.000 \\ 153 & - & - & 100.000 & 99.351 & 98.710 & 98.077 & 97.452 & 96.835 & 96.226 & 95.625 \\ 154 & - & - & - & 100.000 & 99.355 & 98.718 & 98.089 & 97.468 & 96.855 & 96.250 \\ 155 & - & - & - & -- & 100.000 & 99.359 & 98.726 & 98.101 & 97.484 & 96.875 \\ 156 & - & - & - & - & -- & 100.000 & 99.363 & 98.734 & 98.113 & 97.500 \\ 157 & - & - & - & - & -- & - & 100.000 & 99.367 & 98.742 & 98.125 \\ 158 & - & - & - & - & -- & - & - & 100.000 & 99.371 & 98.750 \\ 159 & - & - & - & - & - & - & - & - & 100.000 & 99.375 \\ 160 & - & - & - & - & - & - & - & -- & -- & 100.000\end{array}$




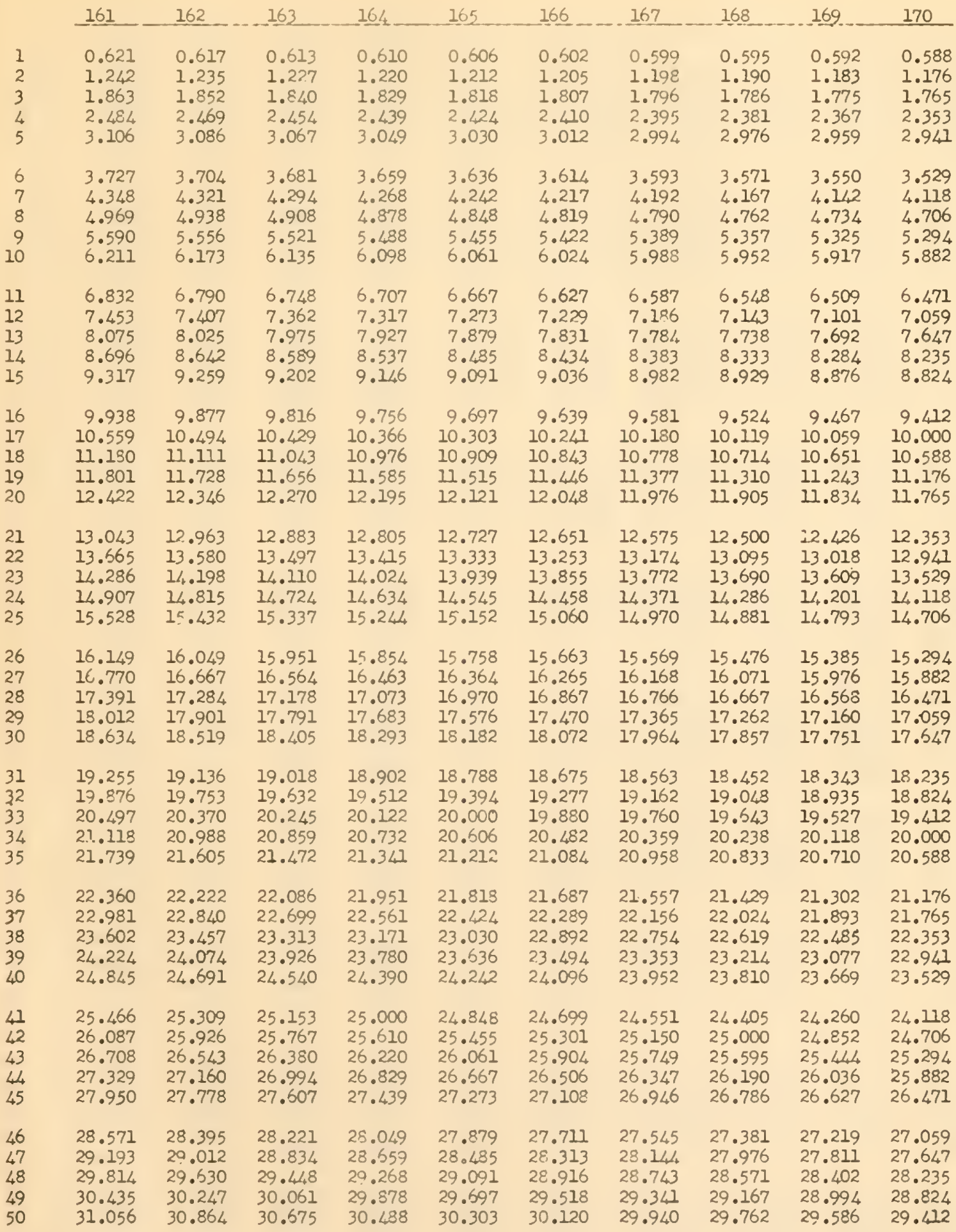




\begin{tabular}{llllllllll}
161 & 162 & 163 & 164 & 165 & 166 & 167 & 168 & 169 & 160 \\
\hline
\end{tabular}

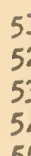

51

52

53

54

55

56

57

58

59

60

61

62

63

64

65

66

67

68

69

70

71

72

73

74

75

76

77

78

79

80

81

82

83

84

85

86

87

88

89

90

91

92

93

94

95

96

97

98

99

100 $\begin{array}{lll}31.677 & 31.481 & 31.288\end{array}$

32.298

32.919

32.099

32.716

31.902

33.540

33.333

34.161

33.951

34.783

34.568

35.404

36.025

35.185

35.802

36.646

36.420

37.037

37.888

38.509

37.654

39.130

38.272

38.889

39.506

39.752

40.373

40.123

40.994

41.615

42.236

40.747

41.358

41.975

42.857

43.478

42.593

43.210

44.099

43.827

44.720

45.342

44.444

45.062

45.679

46.584

46.296

47.205

47.326

46.914

47.531

48.447

48.148

48.765

49.068
49.689

49.383

50.311

50.932

50.000

50.617

51.235

52.174

51.852

52.795

52.469

53.416

54.037

53.086

53.704

54.321

55.280

54.938

55.901

55.556

56.522

56.173

$57.1 / 3$

57.764

58.385

56.790

57.407

58.025

58.642

59.627

59.259

60.248

60.870

59.877

60.494

61.111

62.112

61.728
32.515

33.129

33.742

34.356

34.969

35.583

36.196

36.810

37.423

38.037

38.650

39.264

39.877

40.491

41.104

41.718

42.331

42.945

43.558

44.172

44.785

45.399

46.012

46.626

47.239

47.853

48.466

49.080

49.693

50.307

50.920

51.534

52.147

52.761

53.374

53.988

55.215

55.828

56.442

57.055

57.669

58.282

58.896

59.509

60.123

60.736

61.350
54.601

$\begin{array}{lll}31.098 & 30.909 \quad 30.723\end{array}$

$\begin{array}{lll}31.707 & 31.515 & 31.325\end{array}$

$\begin{array}{lll}32.317 & 32.121 & 31.928\end{array}$

$\begin{array}{lll}32.927 & 32.727 & 32.530\end{array}$

$\begin{array}{lll}33.537 & 33.333 \quad 33.133\end{array}$

\section{$34.146 \quad 33.939 \quad 33.735$}

34.756

35.366

35.976

34.545

34.337

$35.152 \quad 34.940$

$35.758 \quad 35.542$

36.585

36.364

36.145

37.195

$36.970 \quad 36.747$

37.805

37.576

$38.415 \quad 38.182$

39.024

38.789

37.349

37.952

39.634

39.394

38.554

39.157

40.244

40.000

39.759

40.854

41.463

40.606

42.073

41.212

40.361

40.964

41.818

47.566

42.683

42.424

42.169

43.293

43.030

42.77

43.636

43.373

44.512

44.242

43.976

45.122

$44.848 \quad 44.578$

45.732

$45 \cdot 455$

45.181

$46.341 \quad 46.061 \quad 45.783$

$46.951 \quad 46.667$

$47.561 \quad 47.273$

46.386

48.171

47.879

46.988

47.590

48.780

48.485

49.390

49.091

50.000

49.697

50.610

50.303

51.220

50.909

51.515

$48 \lcm{193}$

48.795

49.398

50.000

50.602

51.205

52.439

52.121

51.807

53.049

52.727

52.410

53.012

54.268

53.333

53.939

53.614

54.878

54.545

54.217 

PERCENTAGE TABLES

\begin{tabular}{|c|c|c|c|c|c|c|c|c|c|c|}
\hline & -161 & 162 & 163 & 164 & 165 & 166 & 167 & 168 & 169 & 170 \\
\hline $\begin{array}{l}101 \\
102 \\
103 \\
10 \% \\
105\end{array}$ & $\begin{array}{l}62.733 \\
63.354 \\
63.975 \\
64.596 \\
65.217\end{array}$ & $\begin{array}{l}62.346 \\
62.963 \\
63.580 \\
64.198 \\
64.815\end{array}$ & $\begin{array}{l}61.963 \\
62.577 \\
63.190 \\
63.804 \\
64.417\end{array}$ & $\begin{array}{l}61.585 \\
62.195 \\
62.805 \\
63.415 \\
64.024\end{array}$ & $\begin{array}{l}61.212 \\
61.818 \\
62.424 \\
63.030 \\
63.636\end{array}$ & $\begin{array}{l}60.843 \\
61.446 \\
62.048 \\
62.651 \\
63.253\end{array}$ & $\begin{array}{l}60.479 \\
61.078 \\
61.677 \\
62.275 \\
62.874\end{array}$ & $\begin{array}{l}60.119 \\
60.714 \\
61.310 \\
61.905 \\
62.500\end{array}$ & $\begin{array}{l}59.763 \\
60.355 \\
60.947 \\
61.538 \\
62.130\end{array}$ & $\begin{array}{l}59.412 \\
60.000 \\
60.588 \\
61.176 \\
61.765\end{array}$ \\
\hline $\begin{array}{l}106 \\
107 \\
108 \\
109 \\
110\end{array}$ & $\begin{array}{l}65.839 \\
66.460 \\
67.081 \\
67.702 \\
68.323\end{array}$ & $\begin{array}{l}65.432 \\
66.049 \\
66.667 \\
67.284 \\
67.901\end{array}$ & $\begin{array}{l}65.031 \\
65.644 \\
66.258 \\
66.871 \\
67.485\end{array}$ & $\begin{array}{l}64.634 \\
65.244 \\
65.854 \\
66.463 \\
67.073\end{array}$ & $\begin{array}{l}64.242 \\
64.848 \\
65.455 \\
66.061 \\
66.667\end{array}$ & $\begin{array}{l}63.855 \\
64.458 \\
65.060 \\
65.663 \\
66.265\end{array}$ & $\begin{array}{l}63.473 \\
64.072 \\
64.671 \\
65.269 \\
65.868\end{array}$ & $\begin{array}{l}63.095 \\
63.690 \\
61.286 \\
64.881 \\
65.476\end{array}$ & $\begin{array}{l}62.722 \\
63.314 \\
63.905 \\
64.497 \\
65.089\end{array}$ & $\begin{array}{l}62.353 \\
62.941 \\
63.529 \\
64.118 \\
64.706\end{array}$ \\
\hline $\begin{array}{l}111 \\
112 \\
113 \\
114 \\
115\end{array}$ & $\begin{array}{l}68.944 \\
69.565 \\
70.186 \\
70.807 \\
71.429\end{array}$ & $\begin{array}{l}68.519 \\
69.136 \\
69.753 \\
70.370 \\
70.998\end{array}$ & $\begin{array}{l}68.098 \\
68.712 \\
69.325 \\
69.939 \\
70.552\end{array}$ & $\begin{array}{l}67.683 \\
68.293 \\
68.902 \\
69.512 \\
70.122\end{array}$ & $\begin{array}{l}67.273 \\
67.879 \\
68.485 \\
69.091 \\
69.697\end{array}$ & $\begin{array}{l}66.867 \\
67.470 \\
68.072 \\
68.675 \\
69.277\end{array}$ & $\begin{array}{l}66.467 \\
67.066 \\
67.665 \\
68.263 \\
68.862\end{array}$ & $\begin{array}{l}66.071 \\
66.667 \\
67.262 \\
67.857 \\
68.452\end{array}$ & $\begin{array}{l}65.680 \\
66.272 \\
66.864 \\
67.456 \\
68.047\end{array}$ & $\begin{array}{l}65.294 \\
65.882 \\
66.471 \\
67.059 \\
67.647\end{array}$ \\
\hline $\begin{array}{l}116 \\
117 \\
118 \\
119 \\
120\end{array}$ & $\begin{array}{l}72.050 \\
72.671 \\
73.292 \\
73.913 \\
74.534\end{array}$ & $\begin{array}{l}71.605 \\
72.222 \\
72.840 \\
73.457 \\
74.074\end{array}$ & $\begin{array}{l}71.166 \\
71.779 \\
72.393 \\
73.006 \\
73.620\end{array}$ & $\begin{array}{l}70.732 \\
71.341 \\
71.951 \\
72.561 \\
73.171\end{array}$ & $\begin{array}{l}70.303 \\
70.909 \\
71.515 \\
72.121 \\
72.727\end{array}$ & $\begin{array}{l}69.880 \\
70.482 \\
71.084 \\
71.687 \\
72.289\end{array}$ & $\begin{array}{l}69.461 \\
70.060 \\
70.659 \\
71.257 \\
71.856\end{array}$ & $\begin{array}{l}69.048 \\
69.643 \\
70.238 \\
70.833 \\
71.429\end{array}$ & $\begin{array}{l}68.639 \\
69.231 \\
69.822 \\
70.414 \\
71.006\end{array}$ & $\begin{array}{l}68.235 \\
68.824 \\
69.412 \\
70.000 \\
70.588\end{array}$ \\
\hline $\begin{array}{l}121 \\
122 \\
123 \\
124 \\
125\end{array}$ & $\begin{array}{l}75.155 \\
75,776 \\
76.398 \\
77.019 \\
77.640\end{array}$ & $\begin{array}{l}74.691 \\
75.309 \\
75.926 \\
76.543 \\
77.160\end{array}$ & $\begin{array}{l}74.233 \\
74.847 \\
75.460 \\
76.074 \\
76.687\end{array}$ & $\begin{array}{l}73.780 \\
74.390 \\
75.000 \\
75.610 \\
76.220\end{array}$ & $\begin{array}{l}73.333 \\
73.939 \\
74.545 \\
75.152 \\
75.758\end{array}$ & $\begin{array}{l}72.892 \\
73.494 \\
74.096 \\
74.699 \\
75.301\end{array}$ & $\begin{array}{l}72.455 \\
73.054 \\
73.653 \\
74.251 \\
74.850\end{array}$ & $\begin{array}{l}72.024 \\
72.619 \\
73.214 \\
73.810 \\
74.405\end{array}$ & $\begin{array}{l}71.598 \\
72.189 \\
72.781 \\
73.373 \\
73.964\end{array}$ & $\begin{array}{l}71.176 \\
71.765 \\
72.353 \\
72.941 \\
73.529\end{array}$ \\
\hline $\begin{array}{l}126 \\
127 \\
128 \\
129 \\
130\end{array}$ & $\begin{array}{l}78.251 \\
78.882 \\
79.503 \\
80.124 \\
80.745\end{array}$ & $\begin{array}{l}77.778 \\
75.395 \\
79.012 \\
79.630 \\
80.247\end{array}$ & $\begin{array}{l}77.301 \\
77.914 \\
78.528 \\
79.141 \\
79.755\end{array}$ & $\begin{array}{l}76.829 \\
77.439 \\
78.049 \\
78.659 \\
79.268\end{array}$ & $\begin{array}{l}76.364 \\
76.970 \\
77.576 \\
78.182 \\
78.788\end{array}$ & $\begin{array}{l}75.904 \\
76.506 \\
77.108 \\
77.711 \\
78.313\end{array}$ & $\begin{array}{l}75.449 \\
76.048 \\
76.647 \\
77.246 \\
77.844\end{array}$ & $\begin{array}{l}75.000 \\
75.595 \\
76.190 \\
76.786 \\
77.381\end{array}$ & $\begin{array}{l}74.556 \\
75.148 \\
75.740 \\
76.331 \\
76.923\end{array}$ & $\begin{array}{l}74.118 \\
74.706 \\
75.294 \\
75.882 \\
76.471\end{array}$ \\
\hline $\begin{array}{l}131 \\
132 \\
133 \\
134 \\
135\end{array}$ & $\begin{array}{l}81.366 \\
81.988 \\
82.609 \\
83.230 \\
83.851\end{array}$ & $\begin{array}{l}80.864 \\
81.481 \\
82.099 \\
82.716 \\
83.333\end{array}$ & $\begin{array}{l}80.368 \\
80.982 \\
81.595 \\
82.209 \\
82.822\end{array}$ & $\begin{array}{l}79.878 \\
80.488 \\
81.098 \\
81.707 \\
82.317\end{array}$ & $\begin{array}{l}79.394 \\
80.000 \\
80.606 \\
81.212 \\
81.818\end{array}$ & $\begin{array}{l}78.916 \\
79.518 \\
80.120 \\
80.723 \\
81.325\end{array}$ & $\begin{array}{l}78.443 \\
79.042 \\
79.641 \\
80.240 \\
80.838\end{array}$ & $\begin{array}{l}77.976 \\
78.571 \\
79.167 \\
79.762 \\
80.357\end{array}$ & $\begin{array}{l}77.515 \\
78.107 \\
78.698 \\
79.290 \\
79.882\end{array}$ & $\begin{array}{l}77.059 \\
77.647 \\
78.235 \\
78.824 \\
79.412\end{array}$ \\
\hline $\begin{array}{l}136 \\
137 \\
138 \\
139 \\
140\end{array}$ & $\begin{array}{l}84.472 \\
85.093 \\
85.714 \\
86.335 \\
86.957\end{array}$ & $\begin{array}{l}83.951 \\
84.568 \\
85.185 \\
85.802 \\
86.420\end{array}$ & $\begin{array}{l}83.436 \\
84.049 \\
84.663 \\
85.276 \\
85.890\end{array}$ & $\begin{array}{l}82.927 \\
83.537 \\
84.146 \\
84.756 \\
85.366\end{array}$ & $\begin{array}{l}82.424 \\
83.030 \\
83.636 \\
84.242 \\
81.848\end{array}$ & $\begin{array}{l}81.928 \\
82.530 \\
83.133 \\
83.735 \\
84.337\end{array}$ & $\begin{array}{l}81.437 \\
82.036 \\
82.635 \\
83.234 \\
83.832\end{array}$ & $\begin{array}{l}80.952 \\
81.545 \\
82.143 \\
82.738 \\
83.333\end{array}$ & $\begin{array}{l}80.473 \\
81.065 \\
81.657 \\
82.249 \\
82.840\end{array}$ & $\begin{array}{l}80.000 \\
80.588 \\
81.176 \\
81.765 \\
82.353\end{array}$ \\
\hline $\begin{array}{l}141 \\
142 \\
1 / 3 \\
144 \\
145\end{array}$ & $\begin{array}{l}87.578 \\
88.199 \\
88.820 \\
89.441 \\
90.062\end{array}$ & $\begin{array}{l}87.037 \\
87.654 \\
88.272 \\
88.889 \\
89.506\end{array}$ & $\begin{array}{l}86.503 \\
87.117 \\
87.730 \\
88.344 \\
88.957\end{array}$ & $\begin{array}{l}85.976 \\
86.585 \\
87.195 \\
87.805 \\
88.415\end{array}$ & $\begin{array}{l}85.455 \\
86.061 \\
86.667 \\
87.273 \\
87.879\end{array}$ & $\begin{array}{l}84.940 \\
85.542 \\
86.145 \\
86.747 \\
87.349\end{array}$ & $\begin{array}{l}84.431 \\
85.030 \\
85.629 \\
86.228 \\
86.826\end{array}$ & $\begin{array}{l}83.929 \\
8 \% .524 \\
85.119 \\
85.714 \\
86.310\end{array}$ & $\begin{array}{l}83.432 \\
84.024 \\
84.615 \\
85.207 \\
85.799\end{array}$ & $\begin{array}{l}82.041 \\
83.529 \\
84.118 \\
81.706 \\
85.294\end{array}$ \\
\hline $\begin{array}{l}146 \\
147 \\
148 \\
149 \\
150\end{array}$ & $\begin{array}{l}90.683 \\
91.304 \\
91.925 \\
92.547 \\
93.168\end{array}$ & $\begin{array}{l}90.123 \\
90.741 \\
91.358 \\
91.975 \\
92.593\end{array}$ & $\begin{array}{l}89.571 \\
90.184 \\
90.798 \\
91.411 \\
92.025\end{array}$ & $\begin{array}{l}89.024 \\
89.634 \\
90.244 \\
90.854 \\
91.463\end{array}$ & $\begin{array}{l}88.485 \\
89.091 \\
98.697 \\
90.303 \\
90.909\end{array}$ & $\begin{array}{l}87.952 \\
88.554 \\
89.157 \\
89.759 \\
90.361\end{array}$ & $\begin{array}{l}87.425 \\
88.024 \\
88.623 \\
89.222 \\
89.820\end{array}$ & $\begin{array}{l}86.905 \\
87.500 \\
68.095 \\
88.690 \\
89.256\end{array}$ & $\begin{array}{l}86.391 \\
86.982 \\
87.574 \\
88.166 \\
88.757\end{array}$ & $\begin{array}{l}85.882 \\
86.471 \\
87.059 \\
87.647 \\
88.235\end{array}$ \\
\hline
\end{tabular}



PERCENTAGE. TABLES

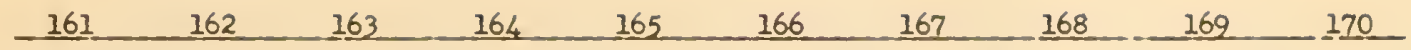

\begin{tabular}{|c|c|c|c|c|c|c|c|c|c|c|}
\hline $\begin{array}{l}151 \\
152 \\
153 \\
154 \\
155\end{array}$ & $\begin{array}{l}93.789 \\
94.410 \\
95.031 \\
95.652 \\
96.273\end{array}$ & $\begin{array}{l}93.210 \\
93.827 \\
94.444 \\
95.062 \\
95.679\end{array}$ & $\begin{array}{l}92.638 \\
93.252 \\
93.865 \\
94.479 \\
95.092\end{array}$ & $\begin{array}{l}92.073 \\
92.683 \\
93.293 \\
93.902 \\
94.512\end{array}$ & $\begin{array}{l}91.515 \\
92.121 \\
92.727 \\
93.333 \\
93.939\end{array}$ & $\begin{array}{l}90.964 \\
91.566 \\
92.169 \\
92.771 \\
93.373\end{array}$ & $\begin{array}{l}90.419 \\
91.018 \\
91.617 \\
92.216 \\
92.814\end{array}$ & $\begin{array}{l}89.881 \\
90.476 \\
91.071 \\
91.667 \\
92.262\end{array}$ & $\begin{array}{l}89.349 \\
89.941 \\
90.533 \\
91.124 \\
91.716\end{array}$ & $\begin{array}{l}88.824 \\
89.412 \\
90.000 \\
90.588 \\
91.176\end{array}$ \\
\hline $\begin{array}{l}59 \\
60\end{array}$ & $\begin{array}{l}96.894 \\
97.516 \\
98.137 \\
98.758 \\
99.379\end{array}$ & $\begin{array}{l}96.296 \\
96.914 \\
97.531 \\
98.148 \\
98.765\end{array}$ & $\begin{array}{l}95.706 \\
96.319 \\
96.933 \\
97.546 \\
98.160\end{array}$ & $\begin{array}{l}95.122 \\
95.732 \\
96.341 \\
96.951 \\
97.561\end{array}$ & $\begin{array}{l}94.545 \\
95.152 \\
95.758 \\
96.364 \\
96.970\end{array}$ & $\begin{array}{l}93.976 \\
94.578 \\
95.181 \\
95.783 \\
96.386\end{array}$ & $\begin{array}{l}93.413 \\
94.012 \\
94.611 \\
95.210 \\
95.808\end{array}$ & $\begin{array}{l}92.857 \\
93.452 \\
94.048 \\
94.643 \\
95.238\end{array}$ & $\begin{array}{l}92.308 \\
92.899 \\
93.491 \\
94.083 \\
94.675\end{array}$ & $\begin{array}{l}91.765 \\
92.353 \\
92.941 \\
93.529 \\
94.118\end{array}$ \\
\hline $\begin{array}{l}161 \\
162 \\
163 \\
164 \\
165\end{array}$ & $\frac{00.000}{-}$ & $\begin{array}{c}99.383 \\
100.000 \\
-\ldots \\
-\end{array}$ & $\begin{array}{r}98.773 \\
99.387 \\
100.000 \\
\ldots \\
-\end{array}$ & $\begin{array}{r}98.171 \\
98.780 \\
99.390 \\
100.000 \\
\end{array}$ & $\begin{array}{r}97.576 \\
98.182 \\
98.788 \\
99.394 \\
100.000\end{array}$ & $\begin{array}{l}96.988 \\
97.590 \\
98.193 \\
98.795 \\
99.398\end{array}$ & $\begin{array}{l}96.407 \\
97.006 \\
97.605 \\
98.204 \\
98.802\end{array}$ & $\begin{array}{l}95.833 \\
96.429 \\
97.024 \\
97.619 \\
98.214\end{array}$ & $\begin{array}{l}95.266 \\
95.858 \\
96.450 \\
97.041 \\
97.633\end{array}$ & $\begin{array}{l}94.706 \\
9.5 .294 \\
95.582 \\
96.471 \\
97.059\end{array}$ \\
\hline $\begin{array}{l}166 \\
167 \\
168 \\
169 \\
170\end{array}$ & $\overline{-}$ & $\begin{array}{c}- \\
-- \\
-\end{array}$ & $\overline{-}$ & $\begin{array}{c}- \\
-- \\
-- \\
-\end{array}$ & $\overline{-}$ & $\begin{array}{c}100.000 \\
- \\
- \\
- \\
-\end{array}$ & $\begin{array}{r}99 \cdot 401 \\
100.000 \\
- \\
-\end{array}$ & $\begin{array}{r}98.810 \\
99.405 \\
100.000 \\
\ldots \\
\ldots\end{array}$ & $\begin{array}{r}98.225 \\
98.817 \\
99.408 \\
100.000 \\
\end{array}$ & $\begin{array}{r}97.647 \\
98.235 \\
98.824 \\
99.412 \\
100.000\end{array}$ \\
\hline
\end{tabular}





\begin{tabular}{|c|c|c|c|c|c|c|c|c|c|}
\hline 17 & 272 & 173 & 174 & 175 & 176 & 177 & 278 & 179. & 180 \\
\hline $\begin{array}{l}29.825 \\
30.409 \\
30.994 \\
31.579 \\
38.144\end{array}$ & $\begin{array}{l}29.651 \\
30.233 \\
30.814 \\
32.395 \\
31.977\end{array}$ & $\begin{array}{l}29.480 \\
30.058 \\
30.636 \\
31.214 \\
31.792\end{array}$ & $\begin{array}{l}29.310 \\
29.885 \\
30.460 \\
31.034 \\
31.609\end{array}$ & $\begin{array}{l}29.143 \\
29.714 \\
30.286 \\
30.857 \\
31.429\end{array}$ & $\begin{array}{l}28.977 \\
29.545 \\
30.114 \\
30.682 \\
31.250\end{array}$ & $\begin{array}{l}28.814 \\
29.379 \\
29.944 \\
30.508 \\
31.073\end{array}$ & $\begin{array}{l}28.652 \\
29.213 \\
29.775 \\
30.337 \\
30.899\end{array}$ & $\begin{array}{l}28.492 \\
29.050 \\
29.609 \\
30.268 \\
30.726\end{array}$ & $\begin{array}{l}28.333 \\
28.889 \\
29.444 \\
30.007 \\
30.556\end{array}$ \\
\hline $\begin{array}{l}32.749 \\
33.333 \\
33.918 \\
34.503 \\
35.088\end{array}$ & $\begin{array}{l}32.558 \\
33.140 \\
33.721 \\
34.302 \\
34.884\end{array}$ & $\begin{array}{l}32.370 \\
32.948 \\
33.526 \\
34.104 \\
34.682\end{array}$ & $\begin{array}{l}32.184 \\
32.759 \\
33.333 \\
33.908 \\
34.483\end{array}$ & $\begin{array}{l}32.000 \\
32.571 \\
33.143 \\
33.714 \\
34.286\end{array}$ & $\begin{array}{l}31.828 \\
32.386 \\
32.955 \\
33.523 \\
34.091\end{array}$ & $\begin{array}{l}31.638 \\
32.203 \\
32.768 \\
33.333 \\
33.898\end{array}$ & $\begin{array}{l}31.461 \\
32.022 \\
32.584 \\
33.146 \\
33.708\end{array}$ & $\begin{array}{l}31.285 \\
31.844 \\
32.402 \\
32: 961 \\
33.520\end{array}$ & $\begin{array}{l}31.211 \\
31.667 \\
32.222 \\
32.778 \\
33.333\end{array}$ \\
\hline $\begin{array}{l}35.673 \\
36.257 \\
36.842 \\
37.427 \\
38.012\end{array}$ & $\begin{array}{l}35.465 \\
36.447 \\
36.628 \\
37.209 \\
37.791\end{array}$ & $\begin{array}{l}35.260 \\
35.838 \\
36.416 \\
36.994 \\
37.572\end{array}$ & $\begin{array}{l}35.057 \\
35.632 \\
36.207 \\
36.782 \\
37.356\end{array}$ & $\begin{array}{l}34.857 \\
35.429 \\
36.000 \\
36.571 \\
37.243\end{array}$ & $\begin{array}{l}34.659 \\
35.227 \\
35.795 \\
36.364 \\
36.932\end{array}$ & $\begin{array}{l}34.463 \\
35.028 \\
35.593 \\
36.158 \\
36.723\end{array}$ & $\begin{array}{l}34.270 \\
34.831 \\
35.393 \\
35.955 \\
36.517\end{array}$ & $\begin{array}{l}34.078 \\
34.637 \\
35.196 \\
35.754 \\
36.313\end{array}$ & $\begin{array}{l}33.889 \\
34.444 \\
35.000 \\
35.556 \\
36.111\end{array}$ \\
\hline $\begin{array}{l}38.596 \\
39.181 \\
39.766 \\
40.351 \\
40.936\end{array}$ & $\begin{array}{l}38.372 \\
38.953 \\
39.535 \\
40.116 \\
40.698\end{array}$ & $\begin{array}{l}38.150 \\
38.728 \\
39.306 \\
39.884 \\
40.462\end{array}$ & $\begin{array}{l}37.931 \\
38.506 \\
39.080 \\
39.655 \\
40.230\end{array}$ & $\begin{array}{l}37.714 \\
38.286 \\
38.857 \\
39.429 \\
40.000\end{array}$ & $\begin{array}{l}37.500 \\
38.068 \\
38.636 \\
39.205 \\
39.713\end{array}$ & $\begin{array}{l}37.288 \\
37.853 \\
38.418 \\
38.983 \\
39.548\end{array}$ & $\begin{array}{l}37.079 \\
37.640 \\
38.202 \\
38.764 \\
39.325\end{array}$ & $\begin{array}{l}36.872 \\
37.430 \\
37.989 \\
38.547 \\
39.106\end{array}$ & $\begin{array}{l}36.667 \\
37.222 \\
37.778 \\
38.333 \\
38.889\end{array}$ \\
\hline $\begin{array}{l}41.520 \\
42.105 \\
42.690 \\
43.275 \\
43.860\end{array}$ & $\begin{array}{l}41.279 \\
41.860 \\
42.442 \\
43.023 \\
43.605\end{array}$ & $\begin{array}{l}41.040 \\
41.618 \\
42.197 \\
42.775 \\
43.353\end{array}$ & $\begin{array}{l}40.805 \\
41.379 \\
41.954 \\
42.529 \\
43.103\end{array}$ & $\begin{array}{l}40.571 \\
41.243 \\
41.724 \\
42.286 \\
42.857\end{array}$ & $\begin{array}{l}40.341 \\
40.909 \\
41.477 \\
42.045 \\
42.614\end{array}$ & $\begin{array}{l}40.213 \\
40.678 \\
41.243 \\
41.808 \\
42.373\end{array}$ & $\begin{array}{l}39.888 \\
40.449 \\
41.011 \\
41.573 \\
42.135\end{array}$ & $\begin{array}{l}39.665 \\
40.223 \\
40.782 \\
41.341 \\
41.899\end{array}$ & $\begin{array}{l}39.414 \\
40.070 \\
40.556 \\
41.111 \\
41.667\end{array}$ \\
\hline $\begin{array}{l}44.444 \\
45.029 \\
45.014 \\
45.199 \\
46.734\end{array}$ & $\begin{array}{l}44.136 \\
44.767 \\
45.349 \\
45.930 \\
46.512\end{array}$ & $\begin{array}{l}43.931 \\
44.509 \\
45.087 \\
45.605 \\
46.243\end{array}$ & $\begin{array}{l}43.678 \\
44.253 \\
44.828 \\
45.402 \\
45.977\end{array}$ & $\begin{array}{l}43.429 \\
44.000 \\
44.571 \\
45.143 \\
45.714\end{array}$ & $\begin{array}{l}43.182 \\
43.750 \\
44.318 \\
44.685 \\
45.455\end{array}$ & $\begin{array}{l}42.938 \\
43.503 \\
44.068 \\
44.633 \\
45.198\end{array}$ & $\begin{array}{l}42.697 \\
43.258 \\
43.820 \\
44.382 \\
44.944\end{array}$ & $\begin{array}{l}42.458 \\
43.017 \\
43.575 \\
44.134 \\
44.693\end{array}$ & $\begin{array}{l}42.222 \\
42.778 \\
43.333 \\
43.889 \\
44.444\end{array}$ \\
\hline $\begin{array}{l}47.368 \\
47.953 \\
48.538 \\
49.123 \\
49.738\end{array}$ & $\begin{array}{l}47.093 \\
47.674 \\
48.250 \\
48.837 \\
49.419\end{array}$ & $\begin{array}{l}46.321 \\
47.399 \\
47.977 \\
43.555 \\
49.133\end{array}$ & $\begin{array}{l}46.552 \\
47.126 \\
47.701 \\
48.276 \\
48.851\end{array}$ & $\begin{array}{l}46.286 \\
46.857 \\
47.429 \\
48.000 \\
48.572\end{array}$ & $\begin{array}{l}46.023 \\
46.591 \\
47.159 \\
47.727 \\
48.295\end{array}$ & $\begin{array}{l}45.763 \\
46.328 \\
46.893 \\
47.458 \\
48.023\end{array}$ & $\begin{array}{l}45.506 \\
46.067 \\
46.629 \\
47.191 \\
47.753\end{array}$ & $\begin{array}{l}45.251 \\
45.810 \\
46.369 \\
46.927 \\
47.486\end{array}$ & $\begin{array}{l}45.000 \\
45.556 \\
46.211 \\
46.667 \\
47.222\end{array}$ \\
\hline $\begin{array}{l}50.292 \\
50.877 \\
51.462 \\
52.047 \\
52.632\end{array}$ & $\begin{array}{l}50.200 \\
50.581 \\
51.163 \\
51.744 \\
52.326\end{array}$ & $\begin{array}{l}49.711 \\
50.289 \\
50.657 \\
51.445 \\
52.023\end{array}$ & $\begin{array}{l}49.425 \\
50.000 \\
50.575 \\
51.149 \\
51.724\end{array}$ & $\begin{array}{l}49.143 \\
49.714 \\
50.286 \\
50.857 \\
51.429\end{array}$ & $\begin{array}{l}48.364 \\
49.432 \\
50.000 \\
50.568 \\
51.136\end{array}$ & $\begin{array}{l}48.588 \\
49.253 \\
49.718 \\
50.282 \\
50.847\end{array}$ & $\begin{array}{l}48.315 \\
48.876 \\
49.438 \\
50.000 \\
50.562\end{array}$ & $\begin{array}{l}48.045 \\
48.603 \\
49.162 \\
49.721 \\
50.279\end{array}$ & $\begin{array}{l}4 . .778 \\
48.333 \\
48.889 \\
49.444 \\
50.000\end{array}$ \\
\hline $\begin{array}{l}53.216 \\
53.801 \\
54.386 \\
54.971 \\
55.556\end{array}$ & $\begin{array}{l}52.907 \\
53.488 \\
54.073 \\
54.651 \\
55.233\end{array}$ & $\begin{array}{l}52.601 \\
53.179 \\
53.757 \\
54.335 \\
54.913\end{array}$ & $\begin{array}{l}52.299 \\
52.874 \\
53.448 \\
54.023 \\
54.598\end{array}$ & $\begin{array}{l}52.000 \\
52.571 \\
53.143 \\
53.714 \\
54.286\end{array}$ & $\begin{array}{l}51.775 \\
52.273 \\
52.841 \\
53.409 \\
53.977\end{array}$ & $\begin{array}{l}51.412 \\
51.977 \\
52.542 \\
53.107 \\
53.672\end{array}$ & $\begin{array}{l}51.124 \\
51.685 \\
52.247 \\
52.809 \\
53.371\end{array}$ & $\begin{array}{l}50.838 \\
51.397 \\
51.955 \\
52.514 \\
53.073\end{array}$ & $\begin{array}{l}50.556 \\
51.111 \\
51.667 \\
52.222 \\
52.778\end{array}$ \\
\hline $\begin{array}{l}56.140 \\
50.725 \\
57.310 \\
57.395 \\
58.430\end{array}$ & $\begin{array}{l}55.614 \\
56.395 \\
56.977 \\
57.558 \\
58.140\end{array}$ & $\begin{array}{l}55.491 \\
56.059 \\
56.647 \\
57.225 \\
57.803\end{array}$ & $\begin{array}{l}55.172 \\
55.747 \\
56.322 \\
56.897 \\
57.472\end{array}$ & $\begin{array}{l}54.857 \\
55.429 \\
56.000 \\
56.571 \\
57.143\end{array}$ & $\begin{array}{l}54.545 \\
55.114 \\
55.682 \\
50.250 \\
56.818\end{array}$ & $\begin{array}{l}54.237 \\
54.802 \\
55.367 \\
55.932 \\
50.497\end{array}$ & $\begin{array}{l}53.933 \\
54.494 \\
55.256 \\
55.618 \\
56.100\end{array}$ & $\begin{array}{l}53.631 \\
54.190 \\
54.749 \\
55.307 \\
55.366\end{array}$ & $\begin{array}{l}53.333 \\
53.889 \\
54.444 \\
55.000 \\
55.556\end{array}$ \\
\hline
\end{tabular}



17 17

\subsection{5}

30.409

30.994

31.579

32.164

32.749

33.333

33.918

34.503

35.088

35.673

$$
36.257
$$

36.842

37.427

38.012

38.596

39.181

39.766

40.351

40.936

41.520

42.105

42.690

43.275

43.360

44.444

45.029

45.614

45.199

46.784

47.368

47.953

48.538

49.123

49.708

50.292

50.377

51.462

52.047

52.632

53.216

53.801

54.386

54.971

55.550

56.140

50.725

57.310

57.395

58.430

172 173 174 175 176 177 178 179 180

$29.651 \quad 29.480 \quad 29.310$

30.233

30.814

31.395

31.977

32.558 33.140

33.721

34.302

34.824

35.465

36.947

36.628

37.209

37.791

38.372

38.953

39.535

40.116

40.698

41.279

41.860

42.442

43.023

43.605

44.136

44.767

45.349

45.930

46.512

47.093

47.674

48.250

46.837

49.419

30.058

30.636

29.885

30.460

$31.214 \quad 31.034$

31.792

31.609

32.370

32.184

32.948

33.526

34.104

32.759

33.333

34.682

33.908

35.260

35.838

36.416

36.994

37.572

36.150

38.728

39.306

39.884

40.462

41.040

41.618

42.197

42.775

43.353

43.931

44.509

45.087

45.665

46.243

46.321

47.399

47.977

43.555

49.133

50.000

49. 711

50.581

51.163

51. 744

50.289

50.657

51.445

52.326

52.023

52.907

53.488

52.601

54.073

54.651

53.179

53.757

54.335

55.233

54.913

55.814

56.395

55.491

56.977

56.059

57.558

50.647

57.225

57.803
35.057

35.632

36.207

36.782

37.356

37.931

38.506

39.080

39.655

40.230

40.805

41.379

41.954

42.529

43.103

43.678

44.253

44.828

45.402

45.977

46.552

47.126

47.701

48.276

48.851

49.425

50.000

50.575

51.149

52.299

52.874

53.448

54.023

54.598

55.172

55.747

56.322

56.897

57.47
51.724

29.143

29.714

30.286

30.857

31.429

28.977

29.545

30.682

31.250

32.000

32.571

33.143

33.714

34.286

31.818

32.386

32.955

33.523

34.091

34.857

34.659

35.429

36.000

36.577

35.227

35.795

37.143

36.932

37. 714

38.286

38.857

39.429

37.500

38.068 

$-17-172 \quad 173 \quad 27$

103

i02

103

104

105

106

107

108

109

110

111

112

213

214

15

116

117

118

119

120

121

122

123

124

125

126

127

128

129

130

132

132

133

13

135

136

137

138

139

140

141

142

143

144

145

146

147

148

249

250
59.064

59.649

60.234

60.819

61.404

61.988

62.573

63.158

63.743

64.327

64. 912

65.497

66.082

66.667

67.251

61.836

68.427

69.006

69.591

70.175

70.760

7.345

7.930

72.515

73.099

73.684

74.269

74.854

75.439

76.023

76.608

77.778

78.363

78.447

79.532

80.117

80.702

81.287

81.871

82.456

83.041

83.626

84.211

34.795

85.380

85.965

86.550

87.134

87.719
77.193
58.721

59.302

59.884

60.465

61.047

6. 628

62.209

62.791

63.372

63.953

64.535

65.116

65.698

66.279

66.860

67.442

68.023

68.605

69.186

69.767

70.349

70.930

71.512

72.093

72.674

73.256

73.837

74.419

75.000

75.581

76.163

76.744

77.326

77.507

78.488

79.070

79.651

80.233

30.814

11. 395

31. 977

82.558

83.140

83. 72 ?

84.302

84. 844

85.465

86.047

86.628

87.209
58.382

58.960

59.538

60.116

60.694

62.272

61.850

62.428

63.006

63.584

64.162

64.740

65.318

65.896

60.474

67.052

67.630

68.208

68.786

69.364

69.942

70.520

R. 098

71.676

72.254

72.832

73.410

73.988

74.566

75.145

75.723

76.301

76.879

77.457

78.035

78.613

79.191

79.769

80.347

80.925

64.368

64.943

65.517

66.092

66.667

67.241

67.826

68.391

68.966

69.540

70.115

70.690

71.204

71.839

72.424

72.989

73.563

74.138

74.733

75.287

75.862

76.437

77.011

77.586

78.161

78.736

79.310

79.385

80.460

81.503

31. .034

82.081

82.659

83.237

61.609

82. 184

62.759

83.815

83.333

84.393

84.971

83.908

35.549

84.433

35.057

36.127

85.632

86.705

175

176

177

178

179

180

57.714

58.286

58.857

59.429

60.000

57.955

58.523

59.091

59.659

57.627

58.192

58.757

59.322

60.227

59.887

60.795

60.452

61.304

61.017

61.932

61.532

62.500

62.147

53.068

62.712

63.636

63.277

64.571

65.143

4. 205

54. 773

63.842

64.407

64.972

65.909

65.537

66.857

65.477 


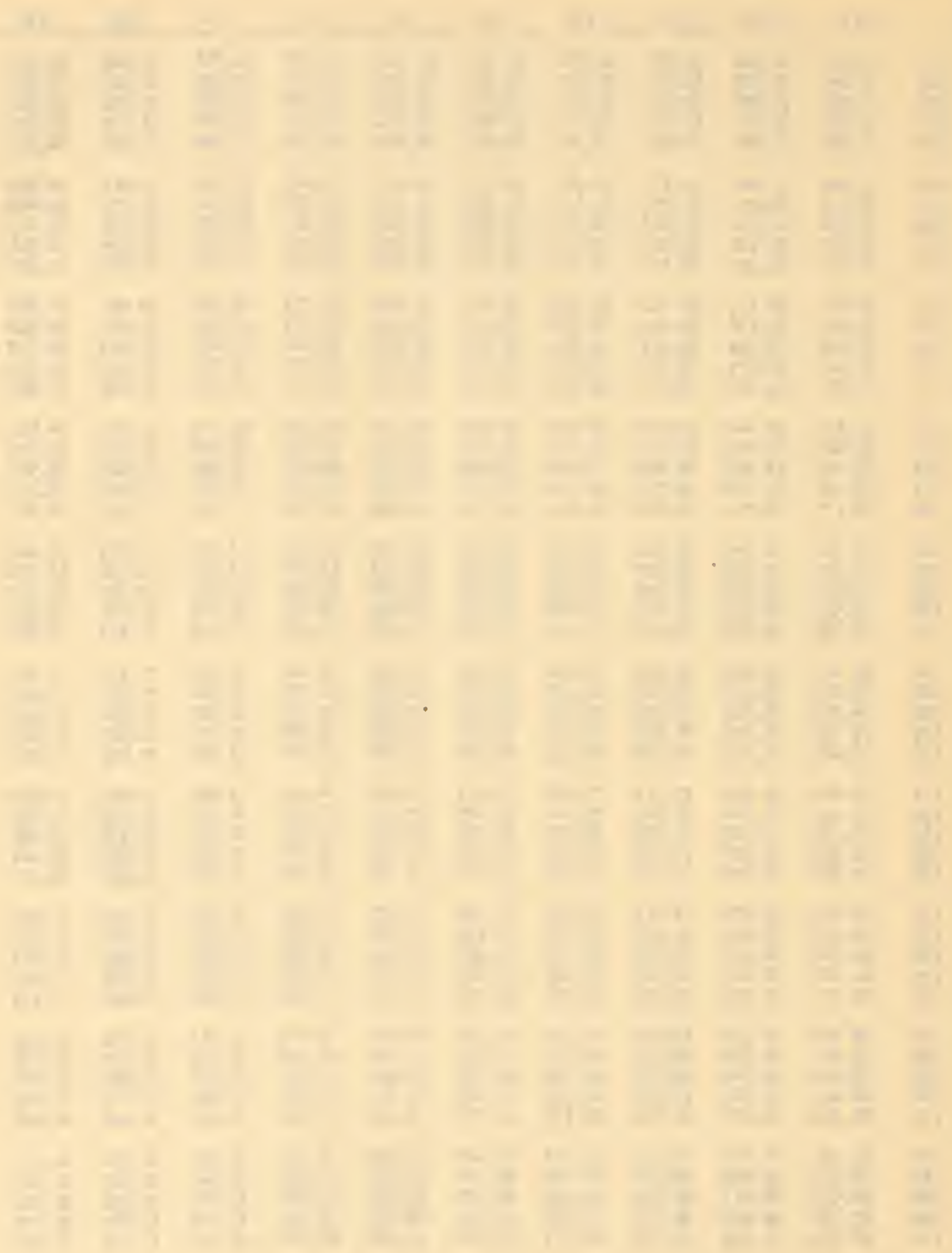





$$
\text { . }
$$




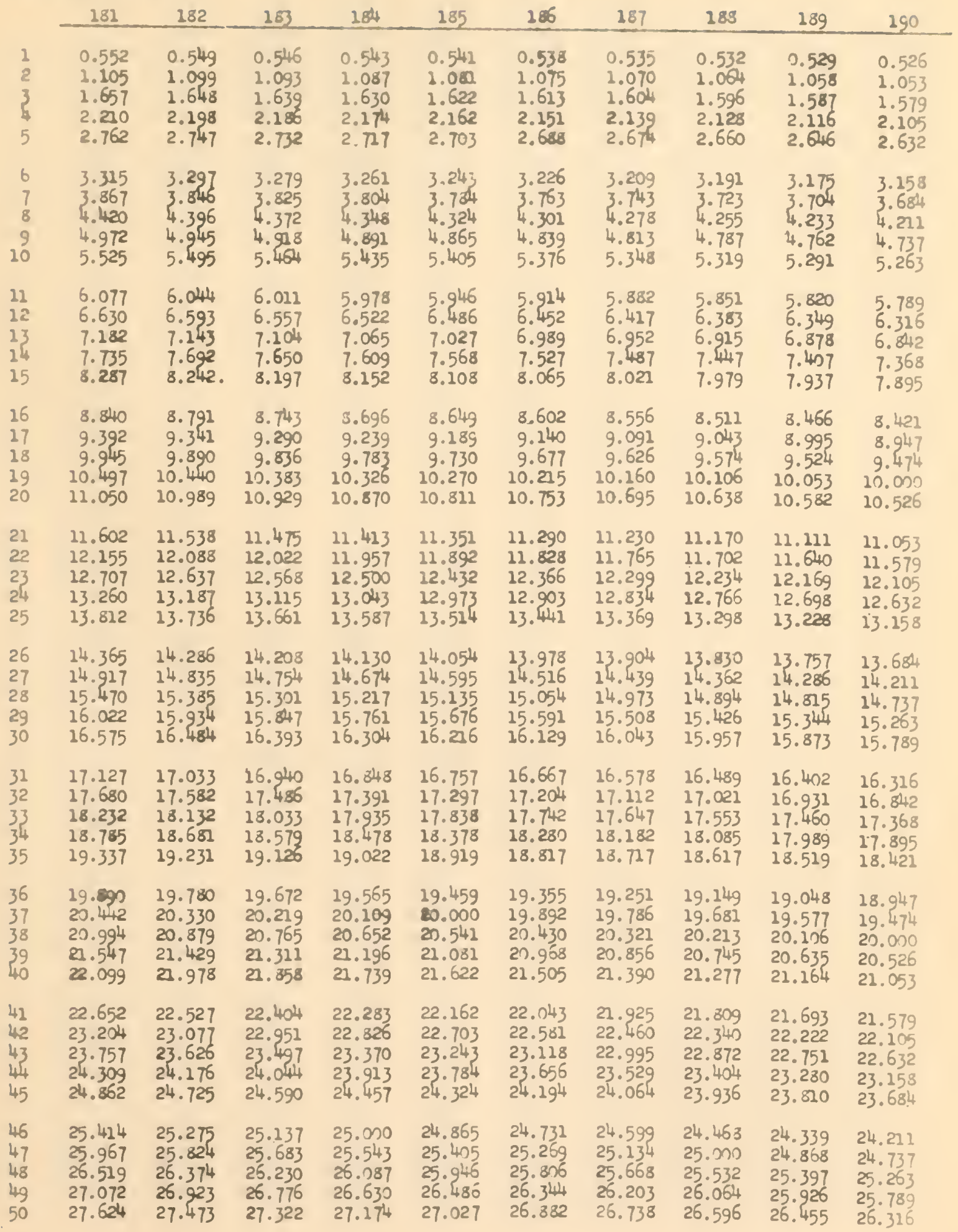





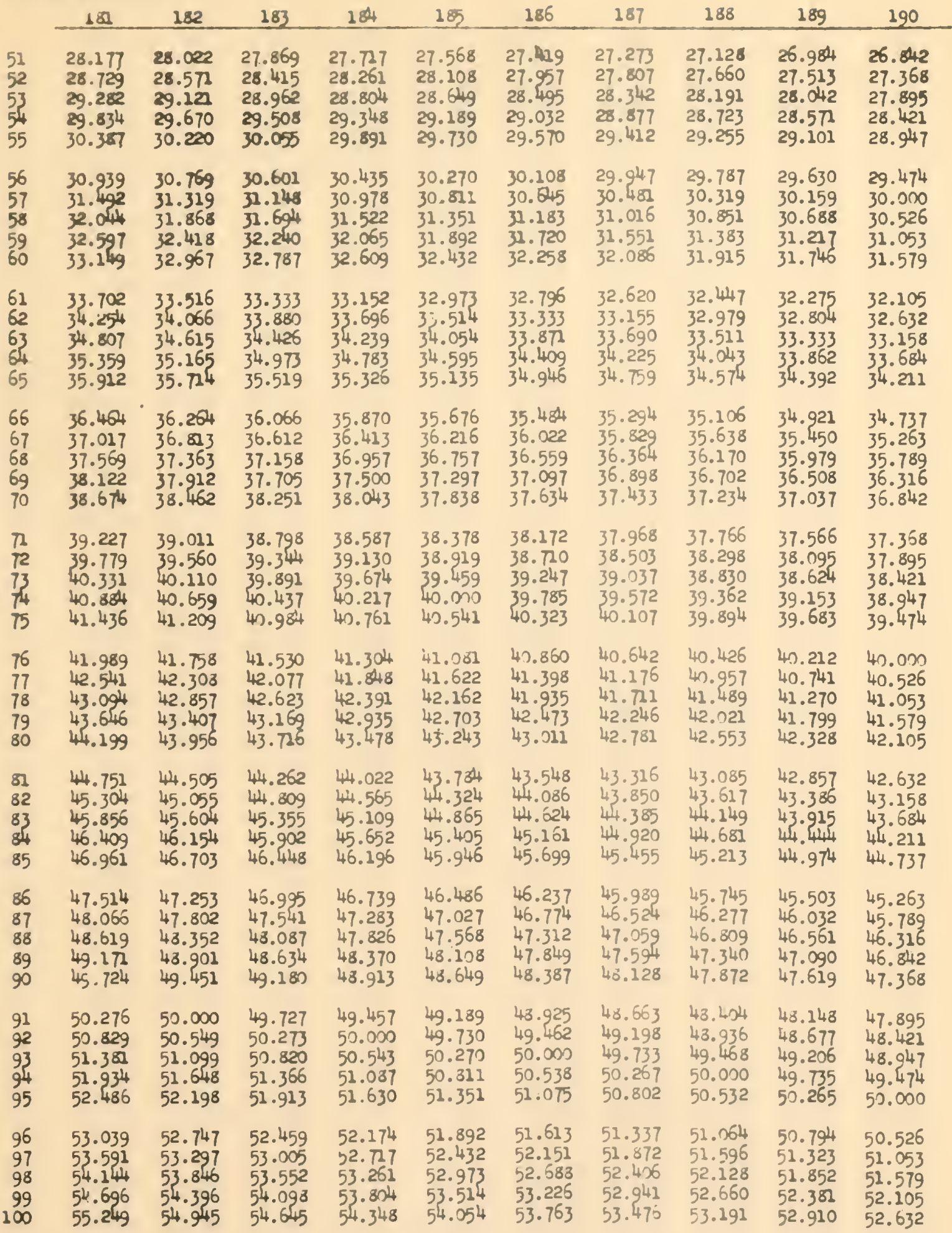





\begin{tabular}{|c|c|c|c|c|c|c|c|c|c|c|}
\hline & 18 & 182 & 183 & 184 & 185 & 186 & 187 & 188 & 189 & 190 \\
\hline $\begin{array}{l}101 \\
102 \\
103 \\
104 \\
105\end{array}$ & $\begin{array}{l}55.801 \\
56.354 \\
56.906 \\
57.459 \\
58.011\end{array}$ & $\begin{array}{l}55.495 \\
56.044 \\
56.593 \\
57.143 \\
57.692\end{array}$ & $\begin{array}{l}55.191 \\
55.738 \\
56.284 \\
56.831 \\
57.377\end{array}$ & $\begin{array}{l}54.891 \\
55.435 \\
55.978 \\
56.522 \\
57.065\end{array}$ & $\begin{array}{l}54.595 \\
55.135 \\
55.676 \\
56.216 \\
56.757\end{array}$ & $\begin{array}{l}54.301 \\
54.839 \\
55.376 \\
55.914 \\
56.452\end{array}$ & $\begin{array}{l}54.011 \\
54.545 \\
55.080 \\
55.615 \\
56.150\end{array}$ & $\begin{array}{l}53.723 \\
54.255 \\
54.787 \\
55.319 \\
55.851\end{array}$ & $\begin{array}{l}53.439 \\
53.968 \\
54.497 \\
55.026 \\
55.556\end{array}$ & $\begin{array}{l}53.158 \\
53.684 \\
54.211 \\
54.737 \\
55.263\end{array}$ \\
\hline $\begin{array}{l}106 \\
107 \\
108 \\
109 \\
110\end{array}$ & $\begin{array}{l}58.564 \\
59.116 \\
59.669 \\
60.221 \\
60.773\end{array}$ & $\begin{array}{l}58.242 \\
58.791 \\
59.341 \\
59.890 \\
60.440\end{array}$ & $\begin{array}{l}57.923 \\
58.470 \\
59.016 \\
59.563 \\
60.109\end{array}$ & $\begin{array}{l}57.609 \\
58.152 \\
58.696 \\
59.239 \\
59.783\end{array}$ & $\begin{array}{l}57.297 \\
57.838 \\
58.378 \\
58.919 \\
59.459\end{array}$ & $\begin{array}{l}56.989 \\
57.527 \\
58.065 \\
58.602 \\
59.140\end{array}$ & $\begin{array}{l}56.684 \\
57.219 \\
57.754 \\
58.289 \\
58.824\end{array}$ & $\begin{array}{l}56.383 \\
56.915 \\
57.447 \\
57.979 \\
58.511\end{array}$ & $\begin{array}{l}56.085 \\
56.614 \\
57.143 \\
57.672 \\
58.201\end{array}$ & $\begin{array}{l}55.789 \\
56.316 \\
56.842 \\
57.368 \\
57.895\end{array}$ \\
\hline $\begin{array}{l}111 \\
112 \\
113 \\
114 \\
115\end{array}$ & $\begin{array}{l}61.326 \\
61.878 \\
62.431 \\
62.983 \\
63.536\end{array}$ & $\begin{array}{l}60.989 \\
61.538 \\
62.088 \\
62.637 \\
63.187\end{array}$ & $\begin{array}{l}60.656 \\
61.202 \\
61.749 \\
62.295 \\
62.842\end{array}$ & $\begin{array}{l}60.326 \\
60.870 \\
61.413 \\
61.957 \\
62.500\end{array}$ & $\begin{array}{l}60.000 \\
60.541 \\
61.081 \\
61.622 \\
62.162\end{array}$ & $\begin{array}{l}59.677 \\
60.215 \\
60.753 \\
61.290 \\
61.828\end{array}$ & $\begin{array}{l}59.358 \\
59.893 \\
60.428 \\
60.963 \\
61.497\end{array}$ & $\begin{array}{l}59.043 \\
59.574 \\
60.106 \\
60.638 \\
61.170\end{array}$ & & $\begin{array}{l}58.421 \\
58.947 \\
59.474 \\
60.000 \\
60.526\end{array}$ \\
\hline $\begin{array}{l}116 \\
117 \\
118 \\
119 \\
120\end{array}$ & $\begin{array}{l}64.088 \\
64.641 \\
65.193 \\
65.746 \\
66.298\end{array}$ & $\begin{array}{l}63.736 \\
64.286 \\
64.835 \\
65.385 \\
65.934\end{array}$ & $\begin{array}{l}63.388 \\
63.934 \\
64.481 \\
65.027 \\
65.574\end{array}$ & $\begin{array}{l}63.043 \\
63.587 \\
64.130 \\
64.674 \\
65.227\end{array}$ & $\begin{array}{l}62.703 \\
63.243 \\
63.784 \\
64.324 \\
64.865\end{array}$ & $\begin{array}{l}62.366 \\
62.903 \\
63.441 \\
63.978 \\
64.516\end{array}$ & $\begin{array}{l}62.032 \\
62.567 \\
63.102 \\
63.636 \\
64.172\end{array}$ & & $\begin{array}{l}61.376 \\
61.905 \\
62.434 \\
52.963 \\
63.492\end{array}$ & $\begin{array}{l}61.053 \\
61.579 \\
62.105 \\
62.632 \\
63.158\end{array}$ \\
\hline $\begin{array}{l}121 \\
122 \\
123 \\
124 \\
125\end{array}$ & $\begin{array}{l}66.851 \\
67.403 \\
67.956 \\
68.508 \\
69.061\end{array}$ & $\begin{array}{l}66.484 \\
67.033 \\
67.582 \\
68.132 \\
68.681\end{array}$ & $\begin{array}{l}66.120 \\
66.667 \\
67.243 \\
67.760 \\
68.306\end{array}$ & $\begin{array}{l}65.761 \\
66.304 \\
66.048 \\
67.391 \\
67.935\end{array}$ & $\begin{array}{l}65.405 \\
65.946 \\
66.486 \\
67.027 \\
67.568\end{array}$ & $\begin{array}{l}65.054 \\
65.591 \\
66.129 \\
66.667 \\
67.204\end{array}$ & $\begin{array}{l}64.706 \\
65.241 \\
65.775 \\
66.310 \\
66.845\end{array}$ & $\begin{array}{l}64.362 \\
44.894 \\
65.426 \\
65.957 \\
66.489\end{array}$ & $\begin{array}{l}64.021 \\
64.550 \\
65.079 \\
65.608 \\
66.138\end{array}$ & $\begin{array}{l}63.684 \\
64.211 \\
64.737 \\
65.263 \\
65.789\end{array}$ \\
\hline $\begin{array}{l}126 \\
127 \\
128 \\
129 \\
130\end{array}$ & $\begin{array}{l}69.613 \\
70.166 \\
70.718 \\
7.271 \\
7.823\end{array}$ & $\begin{array}{l}69.231 \\
69.780 \\
70.330 \\
70.879 \\
71.429\end{array}$ & $\begin{array}{l}68.852 \\
69.399 \\
69.945 \\
70.492 \\
71.038\end{array}$ & & $\begin{array}{l}68.108 \\
68.649 \\
69.189 \\
69.730 \\
70.270\end{array}$ & $\begin{array}{l}67.742 \\
68.280 \\
68.817 \\
69.355 \\
69.892\end{array}$ & $\begin{array}{l}67.380 \\
67.914 \\
68.449 \\
68.984 \\
69.519\end{array}$ & $\begin{array}{l}67.021 \\
67.553 \\
68.085 \\
68.617 \\
69.149\end{array}$ & $\begin{array}{l}66.667 \\
67.196 \\
67.725 \\
68.254 \\
68.783\end{array}$ & $\begin{array}{l}66.316 \\
66.842 \\
67.368 \\
67.895 \\
68.421\end{array}$ \\
\hline $\begin{array}{l}132 \\
133 \\
134 \\
135\end{array}$ & $\begin{array}{l}72.376 \\
72.928 \\
73.481 \\
74.033 \\
74.586\end{array}$ & $\begin{array}{l}71.978 \\
72.527 \\
73.077 \\
73.626 \\
74.176\end{array}$ & $\begin{array}{l}7.585 \\
72.131 \\
72.678 \\
73.224 \\
73.770\end{array}$ & $\begin{array}{l}7.196 \\
7.739 \\
72.283 \\
72.826 \\
73.370\end{array}$ & $\begin{array}{l}70.811 \\
72.351 \\
71.892 \\
72.432 \\
72.973\end{array}$ & $\begin{array}{l}70.430 \\
70.968 \\
71.505 \\
72.043 \\
72.581\end{array}$ & & & $\begin{array}{l}69.312 \\
69.841 \\
70.370 \\
70.899 \\
7 . .429\end{array}$ & $\begin{array}{l}68.947 \\
69.474 \\
70.050 \\
70.526 \\
71.053\end{array}$ \\
\hline $\begin{array}{l}136 \\
137 \\
138 \\
139 \\
140\end{array}$ & $\begin{array}{l}75.138 \\
75.691 \\
76.243 \\
76.796 \\
77.348\end{array}$ & $\begin{array}{l}74.725 \\
75.275 \\
75.824 \\
76.374 \\
76.923\end{array}$ & $\begin{array}{l}74.317 \\
74.863 \\
75.410 \\
75.956 \\
76.503\end{array}$ & $\begin{array}{l}73.913 \\
74.457 \\
75.000 \\
75.543 \\
76.087\end{array}$ & $\begin{array}{l}73.514 \\
74.054 \\
74.595 \\
75.135 \\
75.676\end{array}$ & $\begin{array}{l}73.118 \\
73.656 \\
74.194 \\
74.731 \\
75.259\end{array}$ & $\begin{array}{l}72.727 \\
73.262 \\
73.797 \\
74.332 \\
74.866\end{array}$ & & $\begin{array}{l}71.958 \\
72.487 \\
73.016 \\
73.545 \\
74.074\end{array}$ & $\begin{array}{l}71.579 \\
72.105 \\
72.632 \\
73.158 \\
73.684\end{array}$ \\
\hline $\begin{array}{l}141 \\
142 \\
143 \\
144 \\
145\end{array}$ & $\begin{array}{l}77.901 \\
78.453 \\
79.006 \\
79.558 \\
80.110\end{array}$ & $\begin{array}{l}77.473 \\
78.022 \\
78.571 \\
79.121 \\
79.670\end{array}$ & $\begin{array}{l}77.049 \\
77.596 \\
78.142 \\
78.689 \\
79.235\end{array}$ & $\begin{array}{l}76.630 \\
77.174 \\
77.717 \\
78.261 \\
78.304\end{array}$ & $\begin{array}{l}76.216 \\
76.757 \\
77.297 \\
77.838 \\
78.378\end{array}$ & $\begin{array}{l}75.806 \\
76.344 \\
76.882 \\
77.419 \\
77.957\end{array}$ & $\begin{array}{l}75.401 \\
75.936 \\
76.471 \\
77.005 \\
77.540\end{array}$ & $\begin{array}{l}75.000 \\
75.532 \\
76.064 \\
75.596 \\
77.128\end{array}$ & $\begin{array}{l}74.6 n 3 \\
75.132 \\
75.661 \\
76.190 \\
76.720\end{array}$ & $\begin{array}{l}74.211 \\
74.737 \\
75.263 \\
75.789 \\
76.316\end{array}$ \\
\hline $\begin{array}{l}146 \\
147 \\
148 \\
149 \\
150\end{array}$ & $\begin{array}{l}80.663 \\
81.2215 \\
81.768 \\
82.320 \\
82.873\end{array}$ & $\begin{array}{l}80.220 \\
80.769 \\
81.319 \\
81.868 \\
82.418\end{array}$ & $\begin{array}{l}79.781 \\
80.328 \\
80.874 \\
81.421 \\
81.967\end{array}$ & $\begin{array}{l}79.348 \\
79.891 \\
80.435 \\
80.978 \\
81.522\end{array}$ & $\begin{array}{l}78.919 \\
79.459 \\
80.000 \\
80.541 \\
81.081\end{array}$ & $\begin{array}{l}78.495 \\
79.032 \\
79.570 \\
80.108 \\
80.645\end{array}$ & $\begin{array}{l}78.075 \\
78.610 \\
79.144 \\
79.679 \\
80.214\end{array}$ & $\begin{array}{l}77.660 \\
78.191 \\
78.723 \\
79.255 \\
79.787\end{array}$ & $\begin{array}{l}77.249 \\
77.778 \\
78.307 \\
78.836 \\
79.365\end{array}$ & $\begin{array}{l}76.842 \\
77.368 \\
77.895 \\
78.421 \\
78.947\end{array}$ \\
\hline
\end{tabular}




$\begin{array}{llllllllll}18 & 182 & 183 & 184 & 185 & 186 & 187 & 166 & 159 & 190\end{array}$

\begin{tabular}{|c|c|c|c|c|c|c|c|c|c|c|}
\hline $\begin{array}{l}251 \\
2.52 \\
153 \\
154 \\
155\end{array}$ & $\begin{array}{l}83.425 \\
83.978 \\
84.530 \\
85.083 \\
85.635\end{array}$ & $\begin{array}{l}82.967 \\
83.516 \\
84.066 \\
84.615 \\
85.165\end{array}$ & $\begin{array}{l}82.514 \\
83.060 \\
83.607 \\
84.153 \\
84.699\end{array}$ & $\begin{array}{l}82.065 \\
82.609 \\
83.152 \\
83.696 \\
84.239\end{array}$ & $\begin{array}{l}81.622 \\
82.162 \\
82.703 \\
83.243 \\
83.764\end{array}$ & $\begin{array}{l}81.183 \\
8.720 \\
22.256 \\
82.796 \\
83.333\end{array}$ & $\begin{array}{l}80.749 \\
81.283 \\
81.818 \\
82.353 \\
82.888\end{array}$ & $\begin{array}{l}80.229 \\
80.851 \\
81.383 \\
81.915 \\
82.447\end{array}$ & $\begin{array}{l}79.894 \\
80.423 \\
80.952 \\
81.481 \\
82.011\end{array}$ & $\begin{array}{l}79.474 \\
80.000 \\
80.526 \\
81.053 \\
81.579\end{array}$ \\
\hline 59 & $\begin{array}{l}86.188 \\
86.740 \\
87.293 \\
87.845 \\
88.398\end{array}$ & $\begin{array}{l}85.714 \\
86.264 \\
86.813 \\
87.363 \\
87.912\end{array}$ & $\begin{array}{l}85.246 \\
85.792 \\
86.339 \\
86.885 \\
87.432\end{array}$ & $\begin{array}{l}84.783 \\
85.326 \\
85.870 \\
86.413 \\
86.957\end{array}$ & $\begin{array}{l}84.324 \\
84.865 \\
85.405 \\
85.946 \\
86.486\end{array}$ & $\begin{array}{l}33.87 \\
84.409 \\
84.946 \\
85.484 \\
86.022\end{array}$ & $\begin{array}{l}83.422 \\
83.957 \\
84.492 \\
85.027 \\
85.561\end{array}$ & $\begin{array}{l}82.979 \\
88.511 \\
84.043 \\
84.574 \\
85.106\end{array}$ & $\begin{array}{l}82.540 \\
83.069 \\
83.598 \\
84.127 \\
84.656\end{array}$ & $\begin{array}{l}82.105 \\
82.632 \\
83.158 \\
83.684 \\
84.211\end{array}$ \\
\hline 4 & $\begin{array}{l}38.950 \\
89.503 \\
90.055 \\
90.608 \\
91.160\end{array}$ & $\begin{array}{l}88.462 \\
89.011 \\
89.560 \\
90.117 \\
90.659\end{array}$ & $\begin{array}{l}87.978 \\
88.525 \\
89.071 \\
89.617 \\
90.164\end{array}$ & $\begin{array}{l}87.500 \\
88.043 \\
88.587 \\
89.130 \\
89.674\end{array}$ & $\begin{array}{l}87.027 \\
87.568 \\
88.108 \\
88.649 \\
89.189\end{array}$ & $\begin{array}{l}86.559 \\
87.397 \\
87.634 \\
88.172 \\
88.710\end{array}$ & $\begin{array}{l}86.096 \\
86.631 \\
87.166 \\
87.701 \\
88.235\end{array}$ & $\begin{array}{l}85.638 \\
86.170 \\
86.702 \\
87.234 \\
87.766\end{array}$ & $\begin{array}{l}85.18 \\
85.74 \\
86.243 \\
86.772 \\
87.302\end{array}$ & $\begin{array}{l}84.737 \\
85.263 \\
85.789 \\
86.316 \\
86.842\end{array}$ \\
\hline j9 & $\begin{array}{l}91.713 \\
92.265 \\
92.818 \\
93.370 \\
93.923\end{array}$ & $\begin{array}{l}91.209 \\
91.758 \\
92.308 \\
92.857 \\
93.407\end{array}$ & $\begin{array}{l}90.710 \\
91.257 \\
91.803 \\
92.350 \\
92.896\end{array}$ & $\begin{array}{l}90.217 \\
90.751 \\
91.304 \\
91.848 \\
92.391\end{array}$ & $\begin{array}{l}89.730 \\
90.270 \\
90.811 \\
91.351 \\
91.892\end{array}$ & $\begin{array}{l}89.247 \\
89.785 \\
90.323 \\
90.860 \\
91.398\end{array}$ & $\begin{array}{l}88.770 \\
89.305 \\
89.840 \\
90.374 \\
90.909\end{array}$ & $\begin{array}{l}88.298 \\
88.830 \\
89.362 \\
89.894 \\
90.426\end{array}$ & $\begin{array}{l}87.831 \\
88.360 \\
88.889 \\
89.418 \\
89.947\end{array}$ & $\begin{array}{l}87.368 \\
87.895 \\
88.421 \\
88.947 \\
89.474\end{array}$ \\
\hline 74 & $\begin{array}{l}94.475 \\
95.028 \\
95.580 \\
96.133 \\
96.685\end{array}$ & $\begin{array}{l}93.956 \\
94.505 \\
95.055 \\
95.604 \\
96.154\end{array}$ & $\begin{array}{l}93.443 \\
93.989 \\
94.536 \\
95.082 \\
95.628\end{array}$ & $\begin{array}{l}92.935 \\
93.478 \\
94.022 \\
94.565 \\
95.109\end{array}$ & $\begin{array}{l}92.432 \\
92.973 \\
93.514 \\
94.054 \\
94.595\end{array}$ & $\begin{array}{l}91.935 \\
92.473 \\
93.011 \\
93.548 \\
94.086\end{array}$ & $\begin{array}{l}91.444 \\
91.979 \\
92.513 \\
93.048 \\
93.583\end{array}$ & $\begin{array}{l}90.957 \\
91.489 \\
92.021 \\
92.553 \\
93.085\end{array}$ & $\begin{array}{l}90.476 \\
91.005 \\
91.534 \\
92.063 \\
92.593\end{array}$ & $\begin{array}{l}90.000 \\
90.526 \\
91.053 \\
91.579 \\
92.105\end{array}$ \\
\hline 9 & $\begin{array}{l}97.238 \\
97.790 \\
98.343 \\
98.895 \\
99.448\end{array}$ & $\begin{array}{l}96.703 \\
97.253 \\
97.302 \\
98.352 \\
98.901\end{array}$ & $\begin{array}{l}96.175 \\
96.721 \\
97.268 \\
97.814 \\
98.361\end{array}$ & $\begin{array}{l}95.652 \\
96.196 \\
96.739 \\
97.283 \\
97.826\end{array}$ & $\begin{array}{l}95.135 \\
95.676 \\
96.216 \\
96.757 \\
97.297\end{array}$ & $\begin{array}{l}94.624 \\
95.161 \\
95.699 \\
96.237 \\
96.774\end{array}$ & $\begin{array}{l}94.118 \\
94.652 \\
95.187 \\
95.722 \\
96.257\end{array}$ & $\begin{array}{l}93.617 \\
94.149 \\
94.681 \\
95.213 \\
95.745\end{array}$ & $\begin{array}{l}93.122 \\
93.651 \\
94.180 \\
94.709 \\
95.238\end{array}$ & $\begin{array}{l}92.632 \\
93.158 \\
93.6244 \\
94.211 \\
94.737\end{array}$ \\
\hline 34 & $\begin{array}{c}120.000 \\
--- \\
-- \\
--\end{array}$ & $\begin{array}{c}99.451 \\
100.000 \\
--- \\
---\end{array}$ & $\begin{array}{c}95.907 \\
99.454 \\
100.000 \\
\ldots- \\
\ldots\end{array}$ & $\begin{array}{r}98.370 \\
98.913 \\
99.457 \\
100.000 \\
--\end{array}$ & $\begin{array}{r}97.838 \\
98.378 \\
93.919 \\
99.459 \\
100.000\end{array}$ & $\begin{array}{l}97.312 \\
97.849 \\
98.387 \\
98.925 \\
99.462\end{array}$ & $\begin{array}{l}96.791 \\
97.326 \\
97.861 \\
98.396 \\
98.930\end{array}$ & $\begin{array}{l}96.277 \\
96.809 \\
97.340 \\
97.872 \\
98.404\end{array}$ & $\begin{array}{l}95.767 \\
96.296 \\
96.825 \\
97.354 \\
97.884\end{array}$ & $\begin{array}{l}95.263 \\
95.789 \\
96.316 \\
96.842 \\
97.368\end{array}$ \\
\hline $\begin{array}{l}7 \\
38\end{array}$ & $\begin{array}{l}--- \\
-- \\
--- \\
---\end{array}$ & $\begin{array}{l}-- \\
--- \\
--- \\
---\end{array}$ & $\begin{array}{l}-- \\
-- \\
--\end{array}$ & $\begin{array}{l}--- \\
--- \\
-\infty \\
--\end{array}$ & $\begin{array}{l}--- \\
--- \\
--- \\
--\end{array}$ & $\begin{array}{c}100.000 \\
\ldots \\
-\ldots \\
-\ldots\end{array}$ & $\begin{array}{c}99.465 \\
10 n .000 \\
\cdots \\
\cdots-\end{array}$ & $\begin{array}{c}98.936 \\
99.468 \\
100.000 \\
-.- \\
-.-\end{array}$ & $\begin{array}{c}98.413 \\
98.942 \\
99.471 \\
100.000 \\
-\infty\end{array}$ & $\begin{array}{r}97.895 \\
98.421 \\
98.947 \\
99.474 \\
100.000\end{array}$ \\
\hline
\end{tabular}





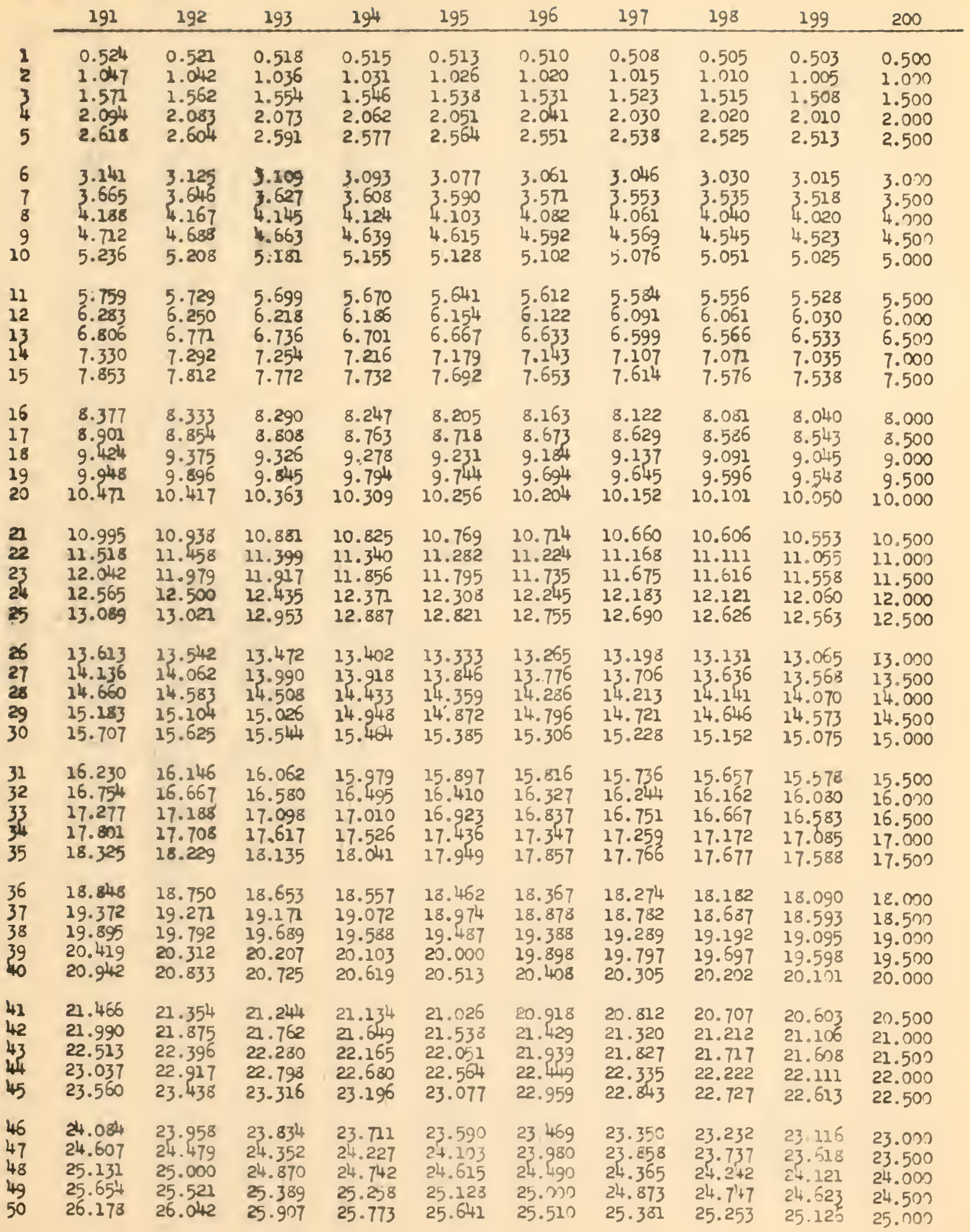


$-19$

द.6. $702 \quad 26.562$

2र. 225

$: 7.749$

28.272

28. 736

29.319

29.343

30.350

30.890

31.2114

61

32.984

33.508

34.031

60

67

5

70

72

73

74

75

76

77

78

79

80

81

82

83

84

85

86

$27.00^{\prime} 3$

2.7 .504

25.125

23.646

29.167

29.688

30.208

30.729

31. 250

31. 771

32.292

32.812

33.333

33.854

34.555

35.079

34.375

35.602

36.126

34.896

35.417

35.938

36.649

35.458

37.173

36.979

37.500

37.095

38.022

38.743

36.542

39.267

39.062

39.791

40.314

40.838

41.361

41.885

39.583

40.104

4n. 625

41.146

41.667

42.408

42.188

42.932

43.455

43.979

42.708

43.229

43.750

44.503

44.277

45.026

44.792

45.550

46.073

45.597

45.312

45.833

46.354

47.120

46.875

47.644

47.396

47.917

48.438

48.691

49. 215

48.958

49.479

50.262

50.000

52.521

51.042

51.309

51.832

51.562
193

194

395

52.083

$\begin{array}{ll}26.455 & 20.269 \\ 20.943 & 20.304 \\ 27.461 & 27.320 \\ 27.979 & 27.035 \\ 26.497 & 20.351\end{array}$

29.016

29.534

28.866

30.052

39.570

31.088

31.606

32.124

32.642

33.161

33.679

34.197

34.715

35.233

35.751

36.209

36.788

37.306

37.824

38.342

33.860

33.378

39.896

40.415

45.933

41.451

41.969

42.487

43.005

43.523

44.041

29.381

29.897

30.412

30.928

31.443

31.959

32.474

32.990

33.505

34.021

34.536

35.052

35.567

36.082

36.598

37.113

37.629

38.144

38.660

39.175

39.691

40.206

4. 722

41.237

41.753

42.268

42.784

43.299

43.814

44.560

45.078

45.596

46.114

46.632

44.330

44.845

45.361

45.876

46.392

47.150

46.907

47.668

43.187

48.705

49.223

47.423

47.938

43.454

48.969

49. 741

50.259

49.485

50.777

51.295

51.813
28. 718

29.231

29.744

30.256

30.769

$31: 282$

31. 795

32.308

32.821

33.333

33.846

34.359

34.872

35.385

35.897

36.410

35.923

37.436

37.949

38.462

38.974

39.487

40.000

40.513

41.026

41.538

42.051

42.564

43.077

43.590

44.103

44.615

26.154
26.667
27.179
27.692
28.205

3,6

26.020

26.531

27.041

27.551

28.061

$28.5 \pi$

29.082

29.592

30.102

30.612

31.122

31.633

32.143

32.653

33.163

33.673

34.184

34.694

35.204

35.714

36.224

36. 735

37.245

37.755

38.265

38.776

39.286

39.796

40.306

40.816

41.327

41.837

42.347

42.857

43.367 



\begin{tabular}{|c|c|c|c|c|c|c|c|c|c|c|}
\hline & 191 & 192 & 193 & 194 & 195 & 196 & 197 & 198 & 199 & 200 \\
\hline $\begin{array}{l}101 \\
102 \\
103 \\
104 \\
105\end{array}$ & $\begin{array}{l}52.880 \\
53.403 \\
53.927 \\
54.450 \\
54.974\end{array}$ & $\begin{array}{l}52.604 \\
53.125 \\
53.646 \\
54.167 \\
54.688\end{array}$ & $\begin{array}{l}52.332 \\
52.850 \\
53.368 \\
53.886 \\
54.404\end{array}$ & $\begin{array}{l}52.062 \\
52.577 \\
53.093 \\
53.608 \\
54.124\end{array}$ & $\begin{array}{l}51.795 \\
52.308 \\
52.821 \\
53.333 \\
53.846\end{array}$ & $\begin{array}{l}51.531 \\
52.041 \\
52.551 \\
53.051 \\
53.571\end{array}$ & $\begin{array}{l}51.269 \\
51.777 \\
52.284 \\
52.792 \\
53.299\end{array}$ & $\begin{array}{l}51.010 \\
51.515 \\
52.020 \\
52.525 \\
53.030\end{array}$ & $\begin{array}{l}50.754 \\
51.256 \\
51.759 \\
52.261 \\
52.704\end{array}$ & $\begin{array}{l}50.500 \\
51.000 \\
51.500 \\
52.000 \\
52.500\end{array}$ \\
\hline $\begin{array}{l}106 \\
107 \\
108 \\
109 \\
110\end{array}$ & $\begin{array}{l}55.497 \\
56.021 \\
56.545 \\
57.068 \\
57.592\end{array}$ & $\begin{array}{l}55.208 \\
55.729 \\
56.250 \\
56.771 \\
57.292\end{array}$ & $\begin{array}{l}54.922 \\
55.440 \\
55.959 \\
56.477 \\
56.995\end{array}$ & $\begin{array}{l}54.639 \\
55.155 \\
55.670 \\
56.186 \\
56.701\end{array}$ & $\begin{array}{l}54.359 \\
54.372 \\
55.385 \\
55.897 \\
55.410\end{array}$ & $\begin{array}{l}54.082 \\
54.592 \\
55.102 \\
55.612 \\
55.122\end{array}$ & $\begin{array}{l}53.807 \\
54.315 \\
54.822 \\
55.330 \\
55.838\end{array}$ & $\begin{array}{l}53.535 \\
54.040 \\
54.545 \\
55.051 \\
55.556\end{array}$ & $\begin{array}{l}53.266 \\
53.769 \\
54.27 \\
54.774 \\
55.276\end{array}$ & $\begin{array}{l}53.000 \\
53.500 \\
54.000 \\
54.500 \\
55.000\end{array}$ \\
\hline $\begin{array}{l}111 \\
112 \\
113 \\
114 \\
115\end{array}$ & $\begin{array}{l}58.115 \\
58.639 \\
59.162 \\
59.686 \\
60.209\end{array}$ & $\begin{array}{l}57.812 \\
58.333 \\
58.854 \\
59.375 \\
59.396\end{array}$ & $\begin{array}{l}57.513 \\
58.051 \\
58.549 \\
59.067 \\
59.585\end{array}$ & $\begin{array}{l}57.216 \\
57.732 \\
58.247 \\
58.763 \\
59.278\end{array}$ & $\begin{array}{l}56.923 \\
57.436 \\
57.949 \\
58.462 \\
53.974\end{array}$ & $\begin{array}{l}56.633 \\
57.243 \\
57.653 \\
58.163 \\
58.673\end{array}$ & $\begin{array}{l}56.345 \\
56.853 \\
57.360 \\
57.868 \\
58.376\end{array}$ & $\begin{array}{l}56.061 \\
56.566 \\
57.071 \\
57.576 \\
58.081\end{array}$ & $\begin{array}{l}55.779 \\
56.281 \\
56.784 \\
57.286 \\
57.789\end{array}$ & $\begin{array}{l}55.500 \\
56.000 \\
56.500 \\
57.020 \\
57.500\end{array}$ \\
\hline $\begin{array}{l}116 \\
117 \\
118 \\
119 \\
120\end{array}$ & $\begin{array}{l}60.733 \\
61.257 \\
61.736 \\
62.304 \\
62.827\end{array}$ & $\begin{array}{l}60.417 \\
60.938 \\
61.458 \\
61.979 \\
62.500\end{array}$ & $\begin{array}{l}60.104 \\
60.622 \\
61.140 \\
61.658 \\
62.176\end{array}$ & $\begin{array}{l}59.794 \\
60.305 \\
60.825 \\
61.340 \\
61.856\end{array}$ & $\begin{array}{l}59.487 \\
60.000 \\
60.513 \\
61.026 \\
61.538\end{array}$ & $\begin{array}{l}59.184 \\
59.694 \\
60.204 \\
60.714 \\
61.224\end{array}$ & $\begin{array}{l}53.883 \\
59.391 \\
59.898 \\
60.406 \\
60.914\end{array}$ & $\begin{array}{l}58.586 \\
59.091 \\
59.596 \\
60.101 \\
60.606\end{array}$ & $\begin{array}{l}58.291 \\
58.794 \\
59.296 \\
59.799 \\
60.302\end{array}$ & $\begin{array}{l}58.000 \\
58.500 \\
59.000 \\
59.500 \\
60.000\end{array}$ \\
\hline $\begin{array}{l}121 \\
122 \\
123 \\
124 \\
125\end{array}$ & $\begin{array}{l}63.351 \\
63.874 \\
64.398 \\
64.921 \\
65.445\end{array}$ & $\begin{array}{l}63.021 \\
63.542 \\
64.062 \\
64.503 \\
65.104\end{array}$ & $\begin{array}{l}52.694 \\
63.212 \\
53.731 \\
64.249 \\
84.767\end{array}$ & $\begin{array}{l}62.371 \\
62.837 \\
53.402 \\
63.918 \\
64.433\end{array}$ & $\begin{array}{l}62.051 \\
62.564 \\
63.077 \\
63.590 \\
64.103\end{array}$ & $\begin{array}{l}61.735 \\
62.245 \\
62.755 \\
63.265 \\
63.776\end{array}$ & $\begin{array}{l}61.421 \\
61.929 \\
62.437 \\
62.944 \\
63.452\end{array}$ & $\begin{array}{l}61.111 \\
61.616 \\
62.121 \\
62.626 \\
63.131\end{array}$ & $\begin{array}{l}60.804 \\
61.307 \\
61.809 \\
62.312 \\
62.814\end{array}$ & $\begin{array}{l}60.500 \\
61.000 \\
61.500 \\
62.000 \\
62.500\end{array}$ \\
\hline $\begin{array}{l}126 \\
127 \\
128 \\
129 \\
130\end{array}$ & $\begin{array}{l}65.959 \\
66.432 \\
67.016 \\
67.539 \\
68.063\end{array}$ & $\begin{array}{l}65.625 \\
66.146 \\
66.667 \\
67.188 \\
67.708\end{array}$ & $\begin{array}{l}65.285 \\
65.803 \\
66.321 \\
66.839 \\
67.358\end{array}$ & $\begin{array}{l}64.948 \\
65.464 \\
65.979 \\
66.495 \\
67.010\end{array}$ & $\begin{array}{l}64.615 \\
65.128 \\
65.641 \\
66.154 \\
66.667\end{array}$ & $\begin{array}{l}64.286 \\
64.796 \\
65.306 \\
65.816 \\
66.327\end{array}$ & $\begin{array}{l}63.959 \\
64.467 \\
64.975 \\
65.482 \\
65.990\end{array}$ & $\begin{array}{l}63.636 \\
64.141 \\
64.646 \\
65.152 \\
65.657\end{array}$ & $\begin{array}{l}63.317 \\
63.819 \\
64.322 \\
64.824 \\
65.327\end{array}$ & $\begin{array}{l}63.000 \\
63.500 \\
64.000 \\
64.500 \\
65.00\end{array}$ \\
\hline $\begin{array}{l}131 \\
132 \\
133 \\
134 \\
135\end{array}$ & $\begin{array}{l}68.536 \\
69.110 \\
69.634 \\
70.157 \\
70.681\end{array}$ & $\begin{array}{l}68.229 \\
68.750 \\
69.27 \\
69.79 c \\
70.312\end{array}$ & $\begin{array}{l}67.876 \\
68.394 \\
68.912 \\
69.430 \\
69.948\end{array}$ & $\begin{array}{l}67.526 \\
68.041 \\
68.557 \\
69.072 \\
69.588\end{array}$ & $\begin{array}{l}67.179 \\
67.692 \\
68.205 \\
68.718 \\
69.231\end{array}$ & $\begin{array}{l}66.837 \\
67.347 \\
67.857 \\
68.367 \\
68.878\end{array}$ & $\begin{array}{l}66.497 \\
67.005 \\
67.513 \\
68.020 \\
68.528\end{array}$ & $\begin{array}{l}66.162 \\
66.667 \\
67.172 \\
67.677 \\
68.182\end{array}$ & $\begin{array}{l}65.829 \\
66.332 \\
66.834 \\
67.337 \\
67.835\end{array}$ & $\begin{array}{l}65.500 \\
66.570 \\
55.500 \\
67.070 \\
57.500\end{array}$ \\
\hline $\begin{array}{l}136 \\
137 \\
138 \\
139 \\
140\end{array}$ & $\begin{array}{l}71.204 \\
71.728 \\
72.251 \\
72.775 \\
73.298\end{array}$ & $\begin{array}{l}70.833 \\
71.354 \\
71.875 \\
72.395 \\
72.917\end{array}$ & $\begin{array}{l}70.466 \\
70.934 \\
71.503 \\
72.021 \\
72.539\end{array}$ & $\begin{array}{l}70.103 \\
70.619 \\
71.134 \\
71.649 \\
72.165\end{array}$ & $\begin{array}{l}60.744 \\
70.256 \\
70.769 \\
71.282 \\
71.795\end{array}$ & $\begin{array}{l}69.388 \\
69.898 \\
70.408 \\
70.918 \\
71.429\end{array}$ & $\begin{array}{l}69.036 \\
69.543 \\
70.051 \\
70.558 \\
71.066\end{array}$ & $\begin{array}{l}68.687 \\
69.192 \\
69.697 \\
70.202 \\
70.707\end{array}$ & $\begin{array}{l}65.342 \\
68.844 \\
69.347 \\
69.849 \\
70.352\end{array}$ & $\begin{array}{l}58.007 \\
68.500 \\
59.000 \\
59.500 \\
70.000\end{array}$ \\
\hline $\begin{array}{l}141 \\
142 \\
143 \\
144 \\
145\end{array}$ & $\begin{array}{l}73.822 \\
74.346 \\
74.369 \\
75.393 \\
75.916\end{array}$ & $\begin{array}{l}73.438 \\
73.953 \\
74.479 \\
75.030 \\
75.521\end{array}$ & $\begin{array}{l}73.057 \\
73.575 \\
74.093 \\
74.611 \\
75.130\end{array}$ & $\begin{array}{l}72.680 \\
73.195 \\
73.711 \\
74.227 \\
74.742\end{array}$ & $\begin{array}{l}72.308 \\
72.821 \\
73.333 \\
73.846 \\
74.359\end{array}$ & $\begin{array}{l}71.939 \\
72.449 \\
72.959 \\
73.469 \\
73.920\end{array}$ & $\begin{array}{l}71.574 \\
72.081 \\
72.589 \\
73.096 \\
73.604\end{array}$ & $\begin{array}{l}71.212 \\
71.717 \\
72.222 \\
72.727 \\
73.232\end{array}$ & $\begin{array}{l}70.854 \\
72.357 \\
71.859 \\
72.362 \\
72.864\end{array}$ & $\begin{array}{l}70.500 \\
7.000 \\
7.500 \\
72.000 \\
72.500\end{array}$ \\
\hline $\begin{array}{l}146 \\
147 \\
148 \\
149 \\
157\end{array}$ & $\begin{array}{l}76.440 \\
76.963 \\
77.487 \\
70.217 \\
70.534\end{array}$ & $\begin{array}{l}76.042 \\
76.562 \\
77.033 \\
77.004 \\
76.125\end{array}$ & $\begin{array}{l}75.648 \\
76.166 \\
76.634 \\
77.202 \\
77.720\end{array}$ & $\begin{array}{l}75.258 \\
75.773 \\
76.289 \\
75.304 \\
77.320\end{array}$ & $\begin{array}{l}74.872 \\
75.385 \\
75.397 \\
76.410 \\
75.923\end{array}$ & $\begin{array}{l}74.490 \\
75.200 \\
75.510 \\
75.020 \\
76.531\end{array}$ & $\begin{array}{l}74.112 \\
74.619 \\
75.127 \\
75.635 \\
75.142\end{array}$ & $\begin{array}{r}73.737 \\
7.242 \\
-14.747 \\
75.253 \\
75.758\end{array}$ & $\begin{array}{l}73.367 \\
73.359 \\
74.372 \\
74.874 \\
75.377\end{array}$ & $\begin{array}{l}73.000 \\
73.500 \\
74.000 \\
74.500 \\
75.000\end{array}$ \\
\hline
\end{tabular}




193

194 195 196 197 198 199 203

151

152

153

154

155

156

157

158

159

$1=0$

161

152

153

164

105

130

107

103

169

170

171

172

173

174

175

176

177

178

179

180

181

132

183

184

155

1 ơ

187

138

189

190

191

192

193

13

195

196

1)7

195

1)

2วว
79.058

79.581

8. . 105

50.628

उ1. 152

78.646

79.167

79.688

80.208

80.729

81.675

81. 250

82.199

81.771

32.723

33.246

82.292

82.812

83.770

83.333

$3 \div .293$

84.817

83.854

85.342

85.854

84.375

84.396

86.387

85.417

55.938

อีó. 911

86.458

87.435

8́. 979

37.958

87.500

38.482

83.021

09.035

83.542

89.529

89.062

-0.052

90.576

39.583

90.104

$91.0 n 9$

90.525

91.623

91.146

92.147

91.667

92.670

92.138

93.194

92.778

93.717

93.223

94.24193 .750

$94.764 \quad 94.271$

95.233

35.812

94.792

96.335

95.312

95.859

95.833

96.354

97.382

95.875

97.976

97.396

93.429

57.917

93.253

30.438

99.470

93.958

100.020

\section{-- 101. ว)}
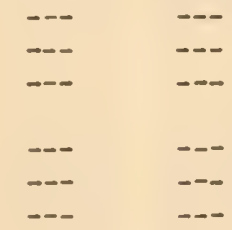

$--$
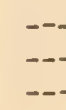

$--$
78.238

78.756

79.275

79.793

50.311

8.). 829

31. 347

31.365

32. 363

32. 402

33.427

83.938

84.456

84. 974

35.492

85. 112

30. 525

37.047

57. 565

83.033

86.601

89.119

09.637

90.155

g0. 674

91.192

91. 710

92.228

92.745

93.204

133. 782

94. 301

94.819

95.337

95.855

77.835

73.351
78.806

79.331

79.897

30.412

30. 928

31. 443

\$1. 959

32.474

32.970

33.525

34.021

34.536

85.352

55.567

35.232

86.593

87.113

87.629

88.144

33.600

39.175

69.691

90.206

90.722

91.237

91.753

92.258

92.784

93.299

93.314

34.330

94.345

95.36 ?

96.373

yb. 570

96.372

97.479

97.927

95., 27

$37.4 \varepsilon 3$

7.933

93.964

3). 432

1)ก. nว

3). 45

10. 259

3y. 45

17). 2\%?
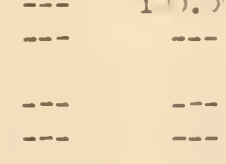

77.435

77.949
70.062

73.974

79.487

ชว.วาว

3). 513

ธ1. 026

81.530

62.251

32.564

อ3. 077

83.590

$3 .+.103$

34.615

35.128

35.641

35.154

86.667

87.179

87.592

83.205

58.718

89.231

89. 744

93.256

0. .759

91.232

1.795

92. 308

92.821

93.333

$13.040^{\circ}$

94. 353

94.372

45.305

35.397

jo. 41 ?

:-23

97.430

37.949

90.452

10. 474

39.487

1า. 3 n 



\begin{tabular}{|c|c|c|c|c|c|c|c|c|c|}
\hline 201 & 202 & 203 & 204 & 205 & 206 & 207 & 208 & 209 & 210 \\
\hline $\begin{array}{l}0.498 \\
0.995 \\
1.493 \\
1.990 \\
2.488\end{array}$ & $\begin{array}{l}0.495 \\
0.990 \\
1.485 \\
1.980 \\
2.475\end{array}$ & $\begin{array}{l}0.493 \\
0.985 \\
1.478 \\
1.970 \\
2.463\end{array}$ & $\begin{array}{l}0.490 \\
0.980 \\
1.472 \\
1.961 \\
2.451\end{array}$ & $\begin{array}{l}0.488 \\
0.976 \\
1.463 \\
1.951 \\
2.439\end{array}$ & $\begin{array}{l}0.485 \\
0.972 \\
1.456 \\
1.942 \\
2.427\end{array}$ & $\begin{array}{l}0.483 \\
0.966 \\
1.449 \\
1.932 \\
2.415\end{array}$ & $\begin{array}{l}0.481 \\
0.962 \\
1.442 \\
1.923 \\
2.404\end{array}$ & $\begin{array}{l}0.478 \\
0.957 \\
1.435 \\
1.914 \\
2.392\end{array}$ & $\begin{array}{l}0.476 \\
0.952 \\
1.429 \\
1.905 \\
2.381\end{array}$ \\
\hline $\begin{array}{l}2.985 \\
3.483 \\
3.980 \\
4.478 \\
4.975\end{array}$ & $\begin{array}{l}2.970 \\
3.465 \\
3.960 \\
4.455 \\
4.950\end{array}$ & $\begin{array}{l}2.956 \\
3.448 \\
3.941 \\
4.433 \\
4.926\end{array}$ & $\begin{array}{l}2.941 \\
3.431 \\
3.922 \\
4.412 \\
4.902\end{array}$ & $\begin{array}{l}2.927 \\
3.415 \\
3.902 \\
4.390 \\
4.878\end{array}$ & $\begin{array}{l}2.913 \\
3.398 \\
3.883 \\
4.369 \\
4.854\end{array}$ & $\begin{array}{l}2.899 \\
3.382 \\
3.865 \\
4.348 \\
4.831\end{array}$ & $\begin{array}{l}2.885 \\
3.365 \\
3.846 \\
4.327 \\
4.808\end{array}$ & $\begin{array}{l}2.87 \\
3.349 \\
3.828 \\
4.306 \\
4.785\end{array}$ & $\begin{array}{l}2.857 \\
3.333 \\
3.810 \\
4.286 \\
4.762\end{array}$ \\
\hline $\begin{array}{l}5.473 \\
5.970 \\
6.468 \\
6.965 \\
7.463\end{array}$ & $\begin{array}{l}5.446 \\
5.941 \\
6.436 \\
6.931 \\
7.426\end{array}$ & $\begin{array}{l}5.419 \\
5.921 \\
6.404 \\
6.897 \\
7.389\end{array}$ & $\begin{array}{l}5.392 \\
5.882 \\
6.373 \\
6.863 \\
7.353\end{array}$ & $\begin{array}{l}5.366 \\
5.854 \\
6.341 \\
6.829 \\
7.327\end{array}$ & $\begin{array}{l}5.340 \\
5.825 \\
6.311 \\
6.796 \\
7.282\end{array}$ & $\begin{array}{l}5.314 \\
5.797 \\
6.280 \\
6.763 \\
7.246\end{array}$ & $\begin{array}{l}5.288 \\
5.759 \\
6.250 \\
6.732 \\
7.212\end{array}$ & $\begin{array}{l}5.263 \\
5.742 \\
6.220 \\
6.699 \\
7.177\end{array}$ & $\begin{array}{l}5.238 \\
5.124 \\
6.190 \\
6.667 \\
7.143\end{array}$ \\
\hline $\begin{array}{l}7.960 \\
8.458 \\
8.955 \\
9.453 \\
9.950\end{array}$ & $\begin{array}{l}7.921 \\
8.426 \\
8.911 \\
9.406 \\
9.901\end{array}$ & $\begin{array}{l}7.882 \\
8.374 \\
8.867 \\
9.360 \\
9.852\end{array}$ & $\begin{array}{l}7.843 \\
8.333 \\
8.824 \\
9.314 \\
9.804\end{array}$ & $\begin{array}{l}7.805 \\
8.293 \\
8.780 \\
9.268 \\
9.756\end{array}$ & $\begin{array}{l}7.767 \\
8.252 \\
8.738 \\
9.223 \\
9.709\end{array}$ & $\begin{array}{l}7.729 \\
8.213 \\
8.696 \\
9.179 \\
9.662\end{array}$ & $\begin{array}{l}7.692 \\
8.173 \\
8.654 \\
9.135 \\
9.615\end{array}$ & $\begin{array}{l}7.656 \\
8.134 \\
8.612 \\
9.091 \\
9.569\end{array}$ & $\begin{array}{l}7.619 \\
8.095 \\
8.571 \\
9.043 \\
9.524\end{array}$ \\
\hline $\begin{array}{l}10.448 \\
10.945 \\
11.443 \\
11.940 \\
12.438\end{array}$ & $\begin{array}{l}10.396 \\
10.891 \\
11.386 \\
11.88 \\
12.376\end{array}$ & $\begin{array}{l}10.345 \\
10.837 \\
11.330 \\
11.823 \\
12.315\end{array}$ & $\begin{array}{l}10.294 \\
10.784 \\
11.275 \\
11.765 \\
12.255\end{array}$ & $\begin{array}{l}10.244 \\
10.732 \\
11.220 \\
11.707 \\
12.195\end{array}$ & $\begin{array}{l}10.194 \\
10.680 \\
11.165 \\
11.650 \\
12.136\end{array}$ & $\begin{array}{l}10.145 \\
10.628 \\
11.111 \\
11.594 \\
12.077\end{array}$ & $\begin{array}{l}10.096 \\
10.577 \\
11.058 \\
11.538 \\
12.019\end{array}$ & $\begin{array}{l}10.048 \\
10.526 \\
11.005 \\
11.483 \\
11.962\end{array}$ & $\begin{array}{l}10.000 \\
10.476 \\
10.952 \\
11.429 \\
11.905\end{array}$ \\
\hline $\begin{array}{l}12.935 \\
13.433 \\
13.930 \\
14.428 \\
14.925\end{array}$ & $\begin{array}{l}12.87 \\
13.366 \\
13.861 \\
14.356 \\
14.851\end{array}$ & $\begin{array}{l}12.808 \\
13.300 \\
13.793 \\
14.236 \\
14.778\end{array}$ & $\begin{array}{l}12.745 \\
13.235 \\
13.725 \\
14.216 \\
14.706\end{array}$ & $\begin{array}{l}12.683 \\
13.17 \\
13.659 \\
14.146 \\
14.634\end{array}$ & $\begin{array}{l}12.621 \\
13.107 \\
13.592 \\
14.078 \\
14.563\end{array}$ & $\begin{array}{l}12.560 \\
13.043 \\
13.527 \\
14.010 \\
14.493\end{array}$ & $\begin{array}{l}12.500 \\
12.961 \\
13.462 \\
13.942 \\
14.423\end{array}$ & $\begin{array}{l}12.440 \\
12.919 \\
13.397 \\
13.876 \\
14.354\end{array}$ & $\begin{array}{l}12.381 \\
12.857 \\
13.333 \\
13.810 \\
14.286\end{array}$ \\
\hline $\begin{array}{l}15.423 \\
15.920 \\
16.418 \\
16.915 \\
17.413\end{array}$ & $\begin{array}{l}15.347 \\
15.842 \\
16.337 \\
16.832 \\
17.327\end{array}$ & $\begin{array}{l}15.271 \\
15.764 \\
16.256 \\
16.749 \\
17.241\end{array}$ & $\begin{array}{l}15.196 \\
15.686 \\
16.176 \\
16.667 \\
17.157\end{array}$ & $\begin{array}{l}15.122 \\
15.610 \\
16.098 \\
16.585 \\
17.073\end{array}$ & $\begin{array}{l}15.049 \\
15.534 \\
16.019 \\
16.505 \\
16.990\end{array}$ & $\begin{array}{l}14.975 \\
15.459 \\
15.942 \\
16.425 \\
16.908\end{array}$ & $\begin{array}{l}14.924 \\
15.385 \\
15.865 \\
16.346 \\
16.827\end{array}$ & $\begin{array}{l}14.833 \\
15.311 \\
15.789 \\
16.268 \\
16.746\end{array}$ & $\begin{array}{l}14.762 \\
15.233 \\
15.714 \\
16.190 \\
16.667\end{array}$ \\
\hline $\begin{array}{l}17.910 \\
18.408 \\
18.905 \\
19.403 \\
19.900\end{array}$ & $\begin{array}{l}17.822 \\
18.317 \\
18.812 \\
19.307 \\
19.802\end{array}$ & $\begin{array}{l}17.734 \\
18.227 \\
18.719 \\
19.222 \\
19.704\end{array}$ & $\begin{array}{l}17.647 \\
18.137 \\
18.627 \\
19.118 \\
19.608\end{array}$ & $\begin{array}{l}17.561 \\
18.049 \\
18.537 \\
19.024 \\
19.512\end{array}$ & $\begin{array}{l}17.476 \\
17.961 \\
18.447 \\
18.932 \\
19.417\end{array}$ & $\begin{array}{l}17.391 \\
17.874 \\
18.357 \\
18.841 \\
19.324\end{array}$ & $\begin{array}{l}17.308 \\
17.788 \\
18.269 \\
18.750 \\
19.231\end{array}$ & $\begin{array}{l}17.225 \\
17.703 \\
18.122 \\
18.650 \\
19.139\end{array}$ & $\begin{array}{l}17.143 \\
17.619 \\
18.095 \\
18.571 \\
19.048\end{array}$ \\
\hline $\begin{array}{l}20.398 \\
20.896 \\
21.393 \\
21.891 \\
22.388\end{array}$ & $\begin{array}{l}20.297 \\
20.792 \\
21.287 \\
21.782 \\
22.277\end{array}$ & $\begin{array}{l}20.197 \\
20.690 \\
21.182 \\
21.675 \\
22.167\end{array}$ & $\begin{array}{l}20.098 \\
20.588 \\
21.078 \\
21.569 \\
22.059\end{array}$ & $\begin{array}{l}20.0 x 0 \\
20.488 \\
20.976 \\
21.463 \\
21.951\end{array}$ & $\begin{array}{l}19.903 \\
20.388 \\
20.874 \\
21.359 \\
21.845\end{array}$ & $\begin{array}{l}19.307 \\
20.290 \\
20.773 \\
21.256 \\
21.739\end{array}$ & $\begin{array}{l}19.712 \\
20.192 \\
20.573 \\
21.154 \\
21.635\end{array}$ & $\begin{array}{l}19.617 \\
20.096 \\
20.574 \\
21.353 \\
21.531\end{array}$ & $\begin{array}{l}19.524 \\
20.510 \\
20.476 \\
20.952 \\
21.429\end{array}$ \\
\hline $\begin{array}{l}22.886 \\
23.383 \\
23.812 \\
24.378 \\
24.876\end{array}$ & $\begin{array}{l}22.772 \\
23.267 \\
23.762 \\
24.257 \\
24.152\end{array}$ & $\begin{array}{l}22.660 \\
23.153 \\
23.645 \\
24.136 \\
24.631\end{array}$ & $\begin{array}{l}22.549 \\
23.039 \\
23.529 \\
24.020 \\
24.510\end{array}$ & $\begin{array}{l}22.439 \\
22.927 \\
23.415 \\
23.902 \\
24.399\end{array}$ & $\begin{array}{l}22.330 \\
22.816 \\
23.301 \\
23.786 \\
24.272\end{array}$ & $\begin{array}{l}22.222 \\
22.705 \\
23.138 \\
23.671 \\
24.155\end{array}$ & $\begin{array}{l}22.115 \\
22.596 \\
23.077 \\
23.558 \\
24.038\end{array}$ & $\begin{array}{l}22.710 \\
22.408 \\
22.967 \\
23.445 \\
23.923\end{array}$ & $\begin{array}{l}21.905 \\
22.381 \\
22.557 \\
23.333 \\
23.810\end{array}$ \\
\hline
\end{tabular}





\begin{tabular}{|c|c|c|c|c|c|c|c|c|c|c|}
\hline & 201 & 202 & 203 & 204 & 205 & 206 & 207 & 208 & 209 & 210 \\
\hline $\begin{array}{l}51 \\
52 \\
53 \\
54 \\
55\end{array}$ & $\begin{array}{l}25.373 \\
25.872 \\
26.368 \\
26.866 \\
27.363\end{array}$ & $\begin{array}{l}25.248 \\
25.743 \\
26.238 \\
26.733 \\
27.228\end{array}$ & $\begin{array}{l}25.123 \\
25.616 \\
26.108 \\
26.601 \\
27.094\end{array}$ & $\begin{array}{l}25.000 \\
25.490 \\
25.980 \\
26.471 \\
26.961\end{array}$ & $\begin{array}{l}24.878 \\
25.366 \\
25.854 \\
26.342 \\
26.829\end{array}$ & $\begin{array}{l}24.757 \\
25.243 \\
25.728 \\
26.214 \\
26.699\end{array}$ & $\begin{array}{l}24.638 \\
25.121 \\
25.604 \\
26.087 \\
26.570\end{array}$ & $\begin{array}{l}24.519 \\
25.000 \\
25.481 \\
25.962 \\
26.442\end{array}$ & $\begin{array}{l}24.402 \\
24.880 \\
25.359 \\
25.837 \\
26.316\end{array}$ & $\begin{array}{l}24.286 \\
24.762 \\
25.238 \\
25.714 \\
26.190\end{array}$ \\
\hline $\begin{array}{l}56 \\
57 \\
58 \\
59 \\
60\end{array}$ & $\begin{array}{l}27.861 \\
28.358 \\
28.856 \\
29.353 \\
29.851\end{array}$ & $\begin{array}{l}27.723 \\
28.218 \\
28.713 \\
29.208 \\
29.703\end{array}$ & $\begin{array}{l}27.586 \\
28.079 \\
28.571 \\
29.064 \\
29.557\end{array}$ & $\begin{array}{l}27.451 \\
27.941 \\
28.431 \\
28.922 \\
29.412\end{array}$ & $\begin{array}{l}27.317 \\
27.805 \\
28.293 \\
28.780 \\
29.268\end{array}$ & $\begin{array}{l}27.184 \\
27.670 \\
28.155 \\
28.641 \\
29.126\end{array}$ & $\begin{array}{l}27.053 \\
27.536 \\
28.019 \\
28.502 \\
28.936\end{array}$ & $\begin{array}{l}26.923 \\
27.404 \\
27.885 \\
28.365 \\
28.846\end{array}$ & $\begin{array}{l}26.794 \\
27.273 \\
27.751 \\
28.230 \\
28.708\end{array}$ & $\begin{array}{l}26.667 \\
27.143 \\
27.619 \\
28.095 \\
28.571\end{array}$ \\
\hline $\begin{array}{l}61 \\
62 \\
63 \\
64 \\
65\end{array}$ & $\begin{array}{l}30.348 \\
30.846 \\
31.343 \\
31.841 \\
32.338\end{array}$ & $\begin{array}{l}30.198 \\
30.693 \\
31.188 \\
31.683 \\
32.178\end{array}$ & $\begin{array}{l}30.049 \\
30.542 \\
31.034 \\
31.527 \\
32.020\end{array}$ & $\begin{array}{l}29.902 \\
30.392 \\
30.882 \\
31.373 \\
31.863\end{array}$ & $\begin{array}{l}29.756 \\
30.244 \\
30.732 \\
31.220 \\
31.797\end{array}$ & $\begin{array}{l}29.612 \\
30.097 \\
30.583 \\
31.068 \\
31.553\end{array}$ & $\begin{array}{l}29.469 \\
25.952 \\
30.435 \\
30.918 \\
31.401\end{array}$ & $\begin{array}{l}29.327 \\
29.808 \\
30.283 \\
32.769 \\
31.250\end{array}$ & $\begin{array}{l}29.187 \\
29.665 \\
30.144 \\
30.622 \\
31.100\end{array}$ & $\begin{array}{l}29.048 \\
29.524 \\
30.000 \\
30.476 \\
30.952\end{array}$ \\
\hline $\begin{array}{l}66 \\
67 \\
68 \\
69 \\
70\end{array}$ & $\begin{array}{l}32.836 \\
33.333 \\
33.831 \\
34.328 \\
34.826\end{array}$ & $\begin{array}{l}32.673 \\
33.168 \\
33.663 \\
34.158 \\
34.653\end{array}$ & $\begin{array}{l}32.512 \\
33.005 \\
33.498 \\
33.990 \\
34.483\end{array}$ & $\begin{array}{l}32.353 \\
32.843 \\
33.333 \\
33.824 \\
34.314\end{array}$ & $\begin{array}{l}32.195 \\
32.683 \\
33.171 \\
33.659 \\
34.146\end{array}$ & $\begin{array}{l}32.039 \\
32.524 \\
33.010 \\
33.495 \\
33.981\end{array}$ & $\begin{array}{l}31.834 \\
32.357 \\
32.850 \\
33.333 \\
33.816\end{array}$ & $\begin{array}{l}31.731 \\
32.212 \\
32.592 \\
33.173 \\
33.654\end{array}$ & $\begin{array}{l}31.579 \\
32.057 \\
32.536 \\
33.014 \\
33.493\end{array}$ & $\begin{array}{l}31.429 \\
31.905 \\
32.381 \\
32.857 \\
33.333\end{array}$ \\
\hline $\begin{array}{l}71 \\
72 \\
73 \\
74 \\
75\end{array}$ & $\begin{array}{l}35.323 \\
35.821 \\
36.318 \\
36.816 \\
37.313\end{array}$ & $\begin{array}{l}35.149 \\
35.644 \\
36.139 \\
36.634 \\
37.129\end{array}$ & $\begin{array}{l}34.975 \\
35.468 \\
35.961 \\
36.453 \\
36.946\end{array}$ & $\begin{array}{l}34.804 \\
35.294 \\
35.784 \\
36.275 \\
36.765\end{array}$ & $\begin{array}{l}34.634 \\
35.122 \\
35.610 \\
36.098 \\
36.585\end{array}$ & $\begin{array}{l}34.466 \\
34.951 \\
35.437 \\
35.922 \\
35.408\end{array}$ & $\begin{array}{l}34.3 n \\
34.733 \\
35.266 \\
35.749 \\
35.232\end{array}$ & $\begin{array}{l}34.135 \\
34.615 \\
35.096 \\
35.577 \\
36.058\end{array}$ & $\begin{array}{l}33.971 \\
34.450 \\
34.928 \\
35.4177 \\
35.835\end{array}$ & $\begin{array}{l}33.810 \\
34.285 \\
34.762 \\
35.238 \\
35.714\end{array}$ \\
\hline $\begin{array}{l}76 \\
77 \\
78 \\
79 \\
80\end{array}$ & $\begin{array}{l}37.811 \\
38.308 \\
38.806 \\
39.303 \\
39.801\end{array}$ & $\begin{array}{l}37.624 \\
38.119 \\
38.614 \\
39.109 \\
39.604\end{array}$ & $\begin{array}{l}37.438 \\
37.931 \\
38.424 \\
38.916 \\
39.409\end{array}$ & $\begin{array}{l}37.255 \\
37.745 \\
38.235 \\
38.725 \\
39.216\end{array}$ & $\begin{array}{l}37.073 \\
37.561 \\
33.049 \\
33.537 \\
39.024\end{array}$ & $\begin{array}{l}36.893 \\
37.379 \\
37.854 \\
38.350 \\
36.835\end{array}$ & $\begin{array}{l}36.715 \\
37.198 \\
37.681 \\
38.164 \\
38.647\end{array}$ & $\begin{array}{l}36.538 \\
37.919 \\
37.500 \\
37.981 \\
38.462\end{array}$ & $\begin{array}{l}36.364 \\
36.842 \\
37.321 \\
37.799 \\
38.278\end{array}$ & $\begin{array}{l}36.190 \\
36.667 \\
37.143 \\
37.619 \\
38.095\end{array}$ \\
\hline $\begin{array}{l}81 \\
82 \\
83 \\
84 \\
85\end{array}$ & $\begin{array}{l}40.299 \\
40.796 \\
41.294 \\
41.791 \\
42.289\end{array}$ & $\begin{array}{l}40.099 \\
40.594 \\
41.089 \\
41.584 \\
42.079\end{array}$ & $\begin{array}{l}39.901 \\
40.394 \\
40.887 \\
41.379 \\
41.872\end{array}$ & $\begin{array}{l}39.706 \\
40.196 \\
40.686 \\
41.176 \\
41.567\end{array}$ & $\begin{array}{l}39.512 \\
40.023 \\
42.486 \\
47.976 \\
41.463\end{array}$ & $\begin{array}{l}39.320 \\
39.806 \\
40.291 \\
40.777 \\
41.262\end{array}$ & $\begin{array}{l}39.130 \\
39.514 \\
40.097 \\
40.580 \\
41.063\end{array}$ & $\begin{array}{l}38.942 \\
39.423 \\
39.904 \\
40.385 \\
40.865\end{array}$ & $\begin{array}{l}38.756 \\
39.234 \\
39.713 \\
40.191 \\
40.670\end{array}$ & $\begin{array}{l}38.571 \\
39.048 \\
39.524 \\
40.570 \\
40.476\end{array}$ \\
\hline $\begin{array}{l}86 \\
87 \\
88 \\
89 \\
90\end{array}$ & $\begin{array}{l}42.786 \\
43.284 \\
43.781 \\
44.279 \\
44.776\end{array}$ & $\begin{array}{l}42.574 \\
43.069 \\
43.564 \\
44.059 \\
44.554\end{array}$ & $\begin{array}{l}42.355 \\
42.857 \\
43.350 \\
43.842 \\
44.335\end{array}$ & $\begin{array}{l}42.157 \\
42.647 \\
43.137 \\
43.627 \\
44.116\end{array}$ & $\begin{array}{l}41.951 \\
42.439 \\
42.927 \\
43.415 \\
43.902\end{array}$ & $\begin{array}{l}41.748 \\
42.233 \\
42.718 \\
43.204 \\
43.689\end{array}$ & $\begin{array}{l}41.546 \\
42.029 \\
42.512 \\
42.995 \\
43.478\end{array}$ & $\begin{array}{l}41.346 \\
41.827 \\
42.308 \\
42.738 \\
43.269\end{array}$ & $\begin{array}{l}41.148 \\
41.627 \\
42.105 \\
42.584 \\
43.062\end{array}$ & $\begin{array}{l}40.952 \\
41.429 \\
41.925 \\
42.381 \\
42.857\end{array}$ \\
\hline $\begin{array}{l}91 \\
92 \\
93 \\
94 \\
95\end{array}$ & $\begin{array}{l}45.274 \\
45.771 \\
46.269 \\
46.766 \\
47.264\end{array}$ & $\begin{array}{l}45.050 \\
45.545 \\
46.040 \\
46.535 \\
47.030\end{array}$ & $\begin{array}{l}44.826 \\
45.320 \\
45.813 \\
46.305 \\
46.798\end{array}$ & $\begin{array}{l}44.608 \\
45.098 \\
45.588 \\
46.078 \\
46.569\end{array}$ & $\begin{array}{l}44.390 \\
44.873 \\
45.366 \\
45.854 \\
46.341\end{array}$ & $\begin{array}{l}44.175 \\
44.603 \\
45.146 \\
45.631 \\
46.117\end{array}$ & $\begin{array}{l}43.961 \\
44.41 .4 \\
44.928 \\
45.411 \\
45.894\end{array}$ & $\begin{array}{l}43.750 \\
44.231 \\
44.712 \\
45.192 \\
45.673\end{array}$ & $\begin{array}{l}43.541 \\
44.019 \\
44.498 \\
44.976 \\
45.455\end{array}$ & $\begin{array}{l}43.333 \\
43.812 \\
44.286 \\
44.762 \\
45.238\end{array}$ \\
\hline $\begin{array}{l}96 \\
97 \\
98 \\
99 \\
100\end{array}$ & $\begin{array}{l}47.761 \\
48.259 \\
48.756 \\
49.254 \\
49.751\end{array}$ & $\begin{array}{l}47.525 \\
48.020 \\
48.515 \\
49.010 \\
49.505\end{array}$ & $\begin{array}{l}47.291 \\
47.783 \\
48.276 \\
48.763 \\
49.261\end{array}$ & $\begin{array}{l}47.059 \\
47.549 \\
48.039 \\
48.529 \\
49.020\end{array}$ & $\begin{array}{l}46.829 \\
47.317 \\
47.825 \\
48.293 \\
48.780\end{array}$ & $\begin{array}{l}46.602 \\
47.087 \\
47.573 \\
48.058 \\
48.544\end{array}$ & $\begin{array}{l}46.377 \\
46.860 \\
47.343 \\
47.826 \\
43.309\end{array}$ & $\begin{array}{l}46.154 \\
46.635 \\
47.115 \\
47.596 \\
48.077\end{array}$ & $\begin{array}{l}45.933 \\
46.411 \\
46.890 \\
47.368 \\
47.847\end{array}$ & $\begin{array}{l}45.714 \\
46.192 \\
46.667 \\
47.143 \\
47.619\end{array}$ \\
\hline
\end{tabular}





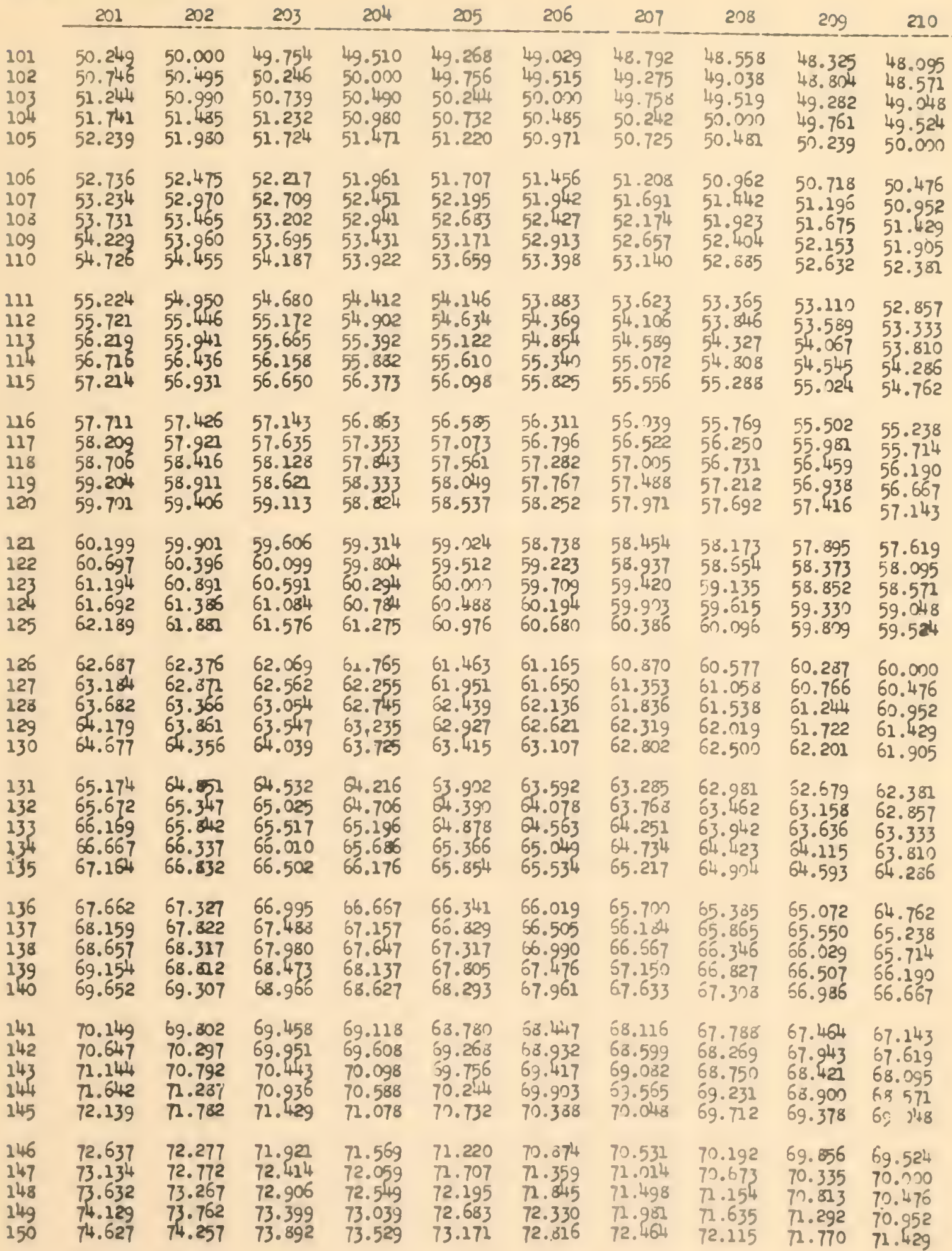


$201 \quad 202$ 203

204

205

206

207

208

809

210

151

75.124

74.752

74.334

74.020

73.659

73.301

72.947

$73.786 \quad 73.430$

76.119

75.743

74.877

74.510

74.146

76.617

76.238

154

77.114

76.733

75.862

75.000

74.634

$75.490 \quad 75.122$

75.980

15.610

14.272

74.757

73.913

75.243

74.396

74.879

76.471

156

77.612

77.228

76.847

78.109

77.723

77.340

76.961

77.833

$159 \quad 79.104$

78.218

78.325

79.602

79.208

78.618

77.451

76.098

76.585

77.073

$78.431 \quad 78.049$

75. 28

76.214

76.699

77.184

77.570

75.362

75. 445

76.329

76.812

77.295

76.922

78.537

73.155

77.778

80. 597

79.310

13.

81. 095

21. 592

ช0.693

79.303

30.296

81.138

80.786

79.902

80.392

79.024

79.512

80.073

00.882

30. 488

166

267

82.537

33.085

33.522

4.080

82.173

61. 773

81.373

30.976

81.863

33.163

83.663

170

44. 577

34. $15 \%$

82. 759

83.251

83.744

82.353

82. 43

83.333

31.463

81.951

32.439

82.927

8.075

171
172

\$. 572

4.65384 .236

$\$ 5.149 \quad 84.729$

86.070

86.567

85.644

क. 222

86.139

85.714

174

87.065

86.634

86.207

176

177

178

179

180

87.562

88.060

87.129

86.700

83.824

84.324

83.415

84.804

85.294

85.784

83.902

84.390

34.878

35.366

73.641

79.126

79.612

3). 097

78.261

78.744

79.227

79.710

$80.583 \quad 80.193$

$81.068 \quad 60.676$

$81.553 \quad 11.159$

$82.039 \quad 81.643$

$82.524 \quad 82.126$

$83.010 \quad 82.609$

$83.495 \quad 83.092$

$33.981 \quad 33.575$

44. 46684.058

24.95184 .541

86.275

35.854

37.192

38.557

89.055

37.624

87.685

83.614

88.177

36.765

87.255

87.745

88.235

86.342

86.829

87.317

87.805

85.437

85.922

86.408

86.893

87.379

85.024

85.507

85.990

86.473

86.957

87.864

90.30

39.604

39.163

182

133

4.4

185

90.547

91.045

90.099

92.594

91.542

91.089

39.655

90.148

90.640

88.725

38.293

89.210

89.706

90.196

38.780

89.268

39.756

90.244

88.350

88.335

89.320

89.805

37.440

87.923

83.406

88.839

89.372

90.291

39.855

92.537

92.079

91.626

91.176

90. 732

91.220

90.777

90.338

92.157

91.707

91.262

93.532

93.069

93.564

92.611

93.103

92.647

92.195

91.148

90.22

91. 304

92.233

91.787

93.17

92.718

92.27

93.627

192

95.025

94.554

94. 039

95.522

95.050

94.581

95.54595 .074

96.04095 .567

94.118

$94.600^{\circ}$

93.659

94.146

95.098

4.634

93.204

93.689

4.175

92.754

93.237

93.720

95.122

4.660

94.203

95.610

95.146

196

198

199

200 



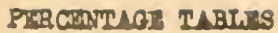

\begin{tabular}{|c|c|c|c|c|c|c|c|c|c|}
\hline 221 & 202 & 203 & 204 & 205 & 206 & 207 & 208 & 209 & 210 \\
\hline 100.000 & 99.505 & 99.015 & 98.529 & 98.049 & 97.573 & $97.1 \mathrm{n}$ & 96.635 & 96.172 & 95.714 \\
\hline -- & 100.000 & 99.507 & 99.020 & 98.537 & 98.058 & 97.585 & 97.115 & 95.651 & 95.190 \\
\hline --- & - & 100.000 & 99.510 & 99.024 & 98.544 & 98.068 & 97.596 & 97.129 & 96.667 \\
\hline --- & $\ldots$ & - & 100.000 & 99.512 & 99.029 & 98.551 & 98.077 & 97.608 & 97.143 \\
\hline$-\infty$ & $-\infty$ & - & $-\infty$ & 20.000 & 99.515 & 99.034 & 98.558 & 98.086 & 97.619 \\
\hline--- & $-\infty$ & - & -- & $-\infty$ & 100.000 & 99.517 & 99.038 & 98.565 & 98. \\
\hline$\ldots$ & $\ldots$ & - & $\infty$ & $\ldots$ & -- & 100.03 & 99.519 & 99.043 & $98.5 \pi$ \\
\hline- & $\cdots$ & -- & $\ldots$ & -- & -- & -- & 100.000 & 99.522 & 99.048 \\
\hline- & $-\infty$ & - & $\cdots$ & $-\infty$ & $-\infty$ & -- & --- & 100.000 & 99.524 \\
\hline & & --- & $-\infty$ & $-\infty$ & - & --- & -- & --- & 5 \\
\hline
\end{tabular}





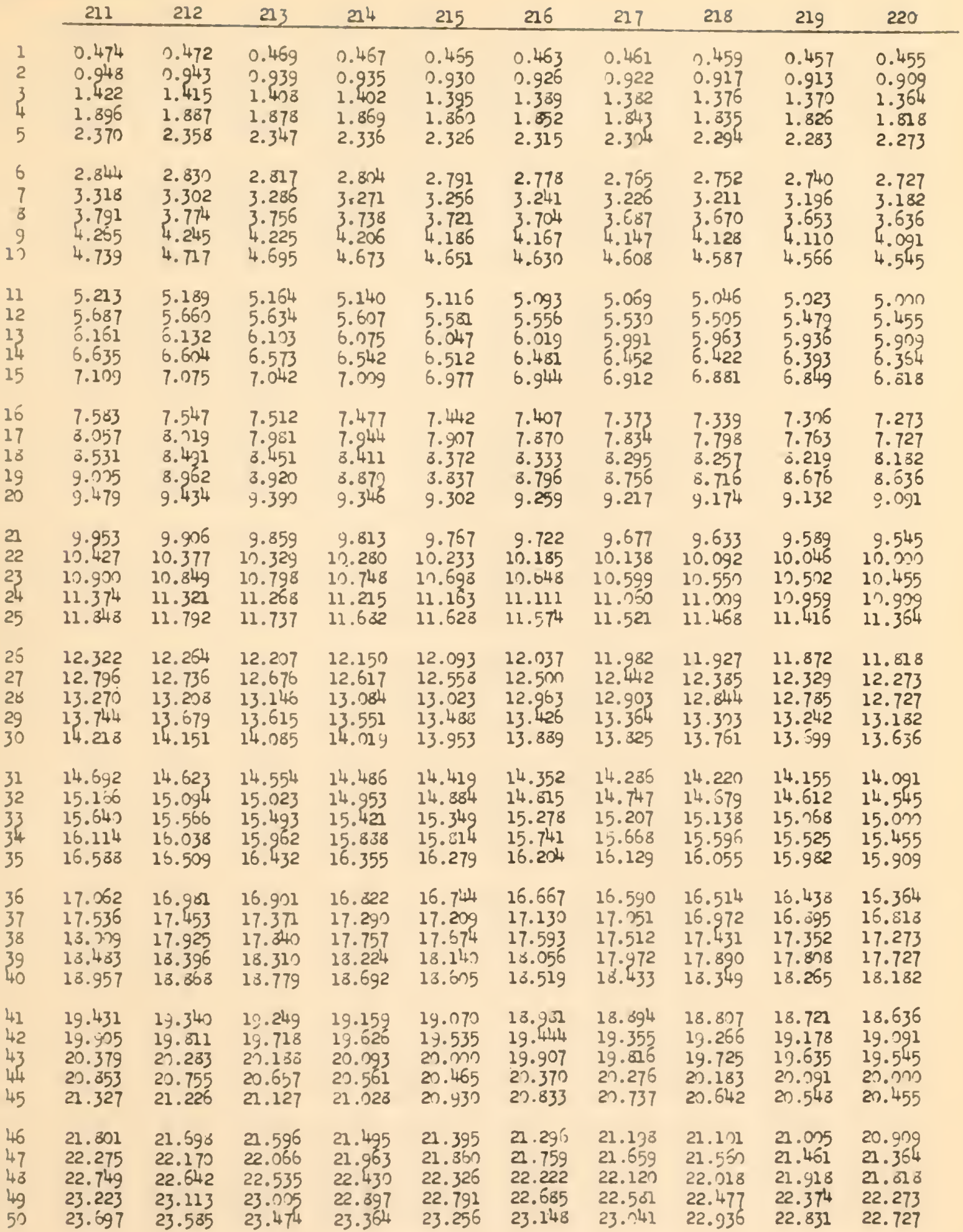




\begin{tabular}{|c|c|c|c|c|c|c|c|c|c|c|}
\hline & 21 & 212 & 213 & 214 & 2215 & 216 & 217 & 218 & 219 & 220 \\
\hline $\begin{array}{l}51 \\
52 \\
53 \\
54 \\
55\end{array}$ & $\begin{array}{l}24.171 \\
24.645 \\
25.118 \\
25.592 \\
26.066\end{array}$ & $\begin{array}{l}24.057 \\
24.528 \\
25.000 \\
25.472 \\
25.943\end{array}$ & $\begin{array}{l}23.944 \\
24.413 \\
24.883 \\
25.352 \\
25.822\end{array}$ & $\begin{array}{l}23.832 \\
24.299 \\
24.766 \\
25.234 \\
25.701\end{array}$ & $\begin{array}{l}23.721 \\
24.186 \\
24.651 \\
25.116 \\
25.581\end{array}$ & $\begin{array}{l}23.611 \\
24.074 \\
24.537 \\
25.050 \\
25.463\end{array}$ & $\begin{array}{l}23.502 \\
23.963 \\
24.424 \\
24.385 \\
25.346\end{array}$ & $\begin{array}{l}23.394 \\
23.853 \\
24.312 \\
24.771 \\
25.229\end{array}$ & $\begin{array}{l}23.288 \\
23.744 \\
24.201 \\
24.658 \\
25.114\end{array}$ & $\begin{array}{l}23.182 \\
23.636 \\
24.091 \\
24.545 \\
25.070\end{array}$ \\
\hline 58 & $\begin{array}{l}26.540 \\
27.014 \\
27.483 \\
27.962 \\
23.436\end{array}$ & $\begin{array}{l}26.415 \\
26.887 \\
27.358 \\
27.830 \\
28.302\end{array}$ & $\begin{array}{l}26.291 \\
26.761 \\
27.230 \\
27.700 \\
28.169\end{array}$ & $\begin{array}{l}26.168 \\
26.636 \\
27.103 \\
27.570 \\
28.037\end{array}$ & $\begin{array}{l}25.047 \\
26.512 \\
26.977 \\
27.442 \\
27.907\end{array}$ & $\begin{array}{l}25.926 \\
26.339 \\
26.352 \\
27.315 \\
27.778\end{array}$ & $\begin{array}{l}25.506 \\
26.267 \\
26.728 \\
27.189 \\
27.650\end{array}$ & $\begin{array}{l}25.588 \\
26.147 \\
26.606 \\
27.064 \\
27.523\end{array}$ & $\begin{array}{l}25.571 \\
26.027 \\
26.484 \\
26.941 \\
27.397\end{array}$ & $\begin{array}{l}25.455 \\
25.999 \\
26.364 \\
26.818 \\
27.273\end{array}$ \\
\hline $\begin{array}{l}62 \\
63 \\
64 \\
65\end{array}$ & $\begin{array}{l}28.910 \\
29.384 \\
29.858 \\
30.332 \\
30.826\end{array}$ & $\begin{array}{l}23.774 \\
29.245 \\
29.717 \\
30.189 \\
30.660\end{array}$ & $\begin{array}{l}28.638 \\
29.108 \\
29.577 \\
30.047 \\
30.516\end{array}$ & $\begin{array}{l}23.505 \\
23.972 \\
29.439 \\
29.907 \\
30.374\end{array}$ & $\begin{array}{l}28.372 \\
23.337 \\
29.302 \\
29.767 \\
30.233\end{array}$ & $\begin{array}{l}28.241 \\
28.704 \\
29.167 \\
29.632 \\
30.093\end{array}$ & $\begin{array}{l}28.111 \\
28.571 \\
29.032 \\
29.493 \\
29.954\end{array}$ & $\begin{array}{l}27.982 \\
28.440 \\
28.899 \\
29.358 \\
29.817\end{array}$ & $\begin{array}{l}27.354 \\
28.311 \\
28.767 \\
29.224 \\
29.580\end{array}$ & $\begin{array}{l}27.727 \\
28.152 \\
28.636 \\
29.091 \\
29.545\end{array}$ \\
\hline $\begin{array}{l}68 \\
69 \\
70\end{array}$ & $\begin{array}{l}31.280 \\
31.754 \\
32.227 \\
32.701 \\
33.175\end{array}$ & $\begin{array}{l}31.132 \\
31.604 \\
32.075 \\
32.547 \\
33.019\end{array}$ & $\begin{array}{l}30.986 \\
31.455 \\
31.925 \\
32.394 \\
32.864\end{array}$ & $\begin{array}{l}30.841 \\
31.303 \\
31.776 \\
32.243 \\
32.710\end{array}$ & $\begin{array}{l}30.698 \\
31.103 \\
31.628 \\
32.093 \\
32.558\end{array}$ & $\begin{array}{l}30.556 \\
31.019 \\
31.481 \\
31.944 \\
32.407\end{array}$ & $\begin{array}{l}32.415 \\
30.375 \\
31.336 \\
31.797 \\
32.258\end{array}$ & $\begin{array}{l}30.275 \\
30.734 \\
31.193 \\
31.651 \\
32.110\end{array}$ & $\begin{array}{l}30.237 \\
30.594 \\
31.050 \\
31.507 \\
31.963\end{array}$ & $\begin{array}{l}30.097 \\
30.455 \\
30.909 \\
31.364 \\
31.818\end{array}$ \\
\hline $\begin{array}{l}71 \\
72 \\
73 \\
74 \\
75\end{array}$ & $\begin{array}{l}33.649 \\
34.123 \\
34.597 \\
35.071 \\
35.545\end{array}$ & $\begin{array}{l}33.491 \\
33.962 \\
34.434 \\
34.906 \\
35.377\end{array}$ & $\begin{array}{l}33.333 \\
33.323 \\
34.272 \\
34.742 \\
35.211\end{array}$ & $\begin{array}{l}33.173 \\
33.645 \\
34.112 \\
34.579 \\
35.347\end{array}$ & $\begin{array}{l}33.023 \\
33.488 \\
33.953 \\
34.419 \\
34.384\end{array}$ & $\begin{array}{l}32.870 \\
33.333 \\
33.796 \\
34.259 \\
34.722\end{array}$ & $\begin{array}{l}32.719 \\
33.180 \\
33.641 \\
34.171 \\
34.562\end{array}$ & $\begin{array}{l}32.569 \\
33.028 \\
33.436 \\
33.945 \\
34.404\end{array}$ & $\begin{array}{l}32.420 \\
32.877 \\
33.333 \\
33.790 \\
34.247\end{array}$ & $\begin{array}{l}32.273 \\
32.727 \\
33.182 \\
33.636 \\
34.091\end{array}$ \\
\hline $\begin{array}{l}76 \\
77 \\
70 \\
79 \\
80\end{array}$ & $\begin{array}{l}36.019 \\
35.493 \\
36.967 \\
37.441 \\
37.915\end{array}$ & $\begin{array}{l}35.849 \\
36.321 \\
36.792 \\
37.264 \\
37.736\end{array}$ & $\begin{array}{l}35.681 \\
36.150 \\
36.620 \\
37.039 \\
37.559\end{array}$ & $\begin{array}{l}35.514 \\
35.951 \\
36.449 \\
36.916 \\
37.383\end{array}$ & $\begin{array}{l}35.347 \\
35.814 \\
36.279 \\
36.745 \\
37.299\end{array}$ & $\begin{array}{l}35.185 \\
35.648 \\
36.111 \\
36.574 \\
37.037\end{array}$ & $\begin{array}{l}35.023 \\
35.434 \\
35.945 \\
36.406 \\
36.365\end{array}$ & $\begin{array}{l}34.362 \\
35.321 \\
35.730 \\
36.239 \\
36.697\end{array}$ & $\begin{array}{l}34.703 \\
35.160 \\
35.615 \\
36.073 \\
36.530\end{array}$ & $\begin{array}{l}34.545 \\
35.000 \\
35.455 \\
35.909 \\
35.364\end{array}$ \\
\hline $\begin{array}{l}81 \\
82 \\
63 \\
84 \\
35\end{array}$ & $\begin{array}{l}33.383 \\
38.363 \\
39.335 \\
39.310 \\
40.234\end{array}$ & $\begin{array}{l}38.208 \\
38.679 \\
39.151 \\
39.623 \\
40.094\end{array}$ & $\begin{array}{l}38.028 \\
38.496 \\
38.967 \\
39.437 \\
39.906\end{array}$ & $\begin{array}{l}37.350 \\
33.310 \\
33.735 \\
39.252 \\
39.720\end{array}$ & $\begin{array}{l}37.674 \\
33.147 \\
33.525 \\
33.077 \\
33.535\end{array}$ & $\begin{array}{l}37.502 \\
37.963 \\
38.426 \\
38.339 \\
39.352\end{array}$ & $\begin{array}{l}37.327 \\
37.736 \\
38.249 \\
38.727 \\
39.171\end{array}$ & $\begin{array}{l}37.156 \\
37.615 \\
38.073 \\
33.532 \\
38.951\end{array}$ & $\begin{array}{l}36.986 \\
37.443 \\
37.900 \\
38.356 \\
38.813\end{array}$ & $\begin{array}{l}36.818 \\
37.273 \\
37.727 \\
36.182 \\
38.635\end{array}$ \\
\hline $\begin{array}{l}36 \\
87 \\
36 \\
89 \\
93\end{array}$ & $\begin{array}{l}40.758 \\
41.232 \\
41.736 \\
42.132 \\
42.654\end{array}$ & $\begin{array}{l}40.566 \\
41.238 \\
41.579 \\
41.931 \\
42.453\end{array}$ & $\begin{array}{l}40.376 \\
40.345 \\
41.315 \\
41.764 \\
42.254\end{array}$ & $\begin{array}{l}40.187 \\
40.654 \\
41.121 \\
41.539 \\
42.356\end{array}$ & $\begin{array}{l}40.070 \\
40.465 \\
40.937 \\
41.395 \\
41.360\end{array}$ & $\begin{array}{l}39.615 \\
40.278 \\
40.741 \\
41.224 \\
41.657\end{array}$ & $\begin{array}{l}39.631 \\
40.792 \\
40.553 \\
41.014 \\
41.475\end{array}$ & $\begin{array}{l}30.450 \\
39.903 \\
40.367 \\
40.826 \\
41.234\end{array}$ & $\begin{array}{l}39.269 \\
39.726 \\
40.183 \\
4 n .639 \\
41.096\end{array}$ & $\begin{array}{l}39.001 \\
39.545 \\
40.30 n \\
40.455 \\
40.909\end{array}$ \\
\hline $\begin{array}{l}91 \\
92 \\
93 \\
34 \\
95\end{array}$ & $\begin{array}{l}43.123 \\
43.502 \\
44.275 \\
44.55 ? \\
45.024\end{array}$ & $\begin{array}{l}42.925 \\
43.336 \\
43.358 \\
44.340 \\
44.311\end{array}$ & $\begin{array}{l}42.723 \\
43.192 \\
43.562 \\
44.131 \\
4.631\end{array}$ & $\begin{array}{l}42.523 \\
42.391 \\
43.453 \\
43.925 \\
44.393\end{array}$ & $\begin{array}{l}42.325 \\
42.791 \\
43.256 \\
43.721 \\
44.136\end{array}$ & $\begin{array}{l}42.139 \\
42.593 \\
43.056 \\
43.519 \\
43.93\end{array}$ & $\begin{array}{l}41.935 \\
42.336 \\
42.857 \\
43.316 \\
43.779\end{array}$ & $\begin{array}{l}41.743 \\
42.272 \\
42.661 \\
43.119 \\
43.573\end{array}$ & $\begin{array}{l}41.553 \\
42.709 \\
42.466 \\
42.922 \\
43.379\end{array}$ & $\begin{array}{l}41.364 \\
41.318 \\
42.273 \\
42.727 \\
43.182\end{array}$ \\
\hline $\begin{array}{l}96 \\
97 \\
93 \\
99 \\
100\end{array}$ & $\begin{array}{l}45.493 \\
45.972 \\
45.445 \\
46.919 \\
47.393\end{array}$ & $\begin{array}{l}45.233 \\
45.755 \\
46.225 \\
46.693 \\
47.170\end{array}$ & $\begin{array}{l}45.270 \\
45.540 \\
46.059 \\
46.479 \\
46.948\end{array}$ & $\begin{array}{l}4.360 \\
45.327 \\
45.734 \\
45.252 \\
46.729\end{array}$ & $\begin{array}{l}44.651 \\
45.116 \\
45.531 \\
46.047 \\
46.512\end{array}$ & $\begin{array}{l}44.444 \\
4.977 \\
45.379 \\
45.333 \\
46.296\end{array}$ & $\begin{array}{l}44.240 \\
44.700 \\
45.151 \\
45.622 \\
46.003\end{array}$ & $\begin{array}{l}44.037 \\
44.495 \\
44.954 \\
45.413 \\
45.372\end{array}$ & $\begin{array}{l}43.836 \\
44.292 \\
44.749 \\
45.205 \\
45.662\end{array}$ & $\begin{array}{l}43.636 \\
44.091 \\
44.545 \\
45.000 \\
45.455\end{array}$ \\
\hline
\end{tabular}




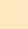


Paramear II or

\begin{tabular}{|c|c|c|c|c|c|c|c|c|c|c|}
\hline & 22. & 27 & 31 & $\Delta$ & 1 & 210 & 27 & 218 & 29 & 220 \\
\hline $\begin{array}{l}101 \\
102 \\
103 \\
104 \\
105\end{array}$ & $\begin{array}{l}47.671 \\
48.312 \\
48.315 \\
49.209 \\
49.763\end{array}$ & $\begin{array}{l}41.6= \\
4 . .113 \\
1 . .5=5 \\
49.057 \\
29.528\end{array}$ & 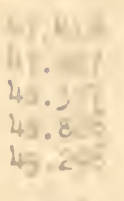 & 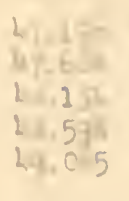 & $\begin{array}{l}16,17 \\
47-512 \\
47.07 \\
4.372 \\
1.837\end{array}$ & $\begin{array}{l}4.721 \\
47.221 \\
47.681 \\
48.148 \\
48.611\end{array}$ & 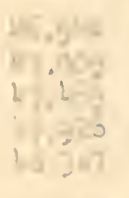 & $\begin{array}{l}26.330 \\
46.789 \\
47.218 \\
47.706 \\
1.8 .165\end{array}$ & $\begin{array}{l}46.119 \\
46.575 \\
47.032 \\
47.489 \\
47.945\end{array}$ & $\begin{array}{l}45.929 \\
46.364 \\
46.818 \\
47.273 \\
47.727\end{array}$ \\
\hline $\begin{array}{l}06 \\
07 \\
08 \\
09 \\
1.5\end{array}$ & $\begin{array}{l}50.237 \\
50.711 \\
51.115 \\
51.659 \\
52.133\end{array}$ & $\begin{array}{l}50.03 n \\
0.472 \\
50.943 \\
51.415 \\
51.837\end{array}$ & $\begin{array}{l}4.175 \\
50.235 \\
50.704 \\
51.174 \\
51.613\end{array}$ & $\begin{array}{l}49.533 \\
53.077 \\
57.2 \\
50.215 \\
0.4\end{array}$ & $\begin{array}{l}1.322 \\
49.757 \\
3.233 \\
90.693 \\
41.153\end{array}$ & $\begin{array}{l}43.074 \\
49.537 \\
50.237 \\
50.463 \\
57.926\end{array}$ & 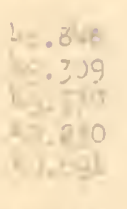 & $\begin{array}{l}16.624 \\
1=.083 \\
4.541 \\
37.777 \\
3.2=9\end{array}$ & $\begin{array}{l}48.402 \\
48.058 \\
49.315 \\
40.772 \\
50.228\end{array}$ & $\begin{array}{l}48.182 \\
48.636 \\
49.091 \\
49.545 \\
50.0 n\end{array}$ \\
\hline $\begin{array}{l}11 \\
12 \\
13 \\
14 \\
13\end{array}$ & $\begin{array}{l}52.501 \\
53.081 \\
3.07 \\
54.728 \\
3 . .73\end{array}$ & $\begin{array}{r}2.358 \\
52.830 \\
51.302 \\
5.774 \\
4.245\end{array}$ & $\begin{array}{l}52.113 \\
52.18 \\
33.058 \\
3.512 \\
53.42\end{array}$ & 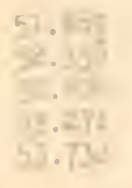 & $\begin{array}{r}1.038 \\
.293 \\
7.553 \\
3.023 \\
3.408\end{array}$ & $\begin{array}{l}51.30 \\
51.852 \\
52.315 \\
52.776 \\
53.241\end{array}$ & $\begin{array}{l}\frac{31.25}{92} \cdot 13 \\
52.13 \\
52.14\end{array}$ & $\begin{array}{r}50.917 \\
51.376 \\
.835 \\
2.294 \\
26.152\end{array}$ & $\begin{array}{l}50.605 \\
51.142 \\
51.598 \\
52.055 \\
5 c .11\end{array}$ & $\begin{array}{l}50.455 \\
50.929 \\
51.354 \\
51.813 \\
52.275\end{array}$ \\
\hline $\begin{array}{l}16 \\
17 \\
13 \\
19 \\
20\end{array}$ & $\begin{array}{l}54.970 \\
55.450 \\
55.24 \\
50.393 \\
56.37\end{array}$ & $\begin{array}{l}54.717 \\
55.139 \\
5.650 \\
30.132 \\
56.604\end{array}$ & $\begin{array}{l}3.25 \\
5 . .730 \\
55.520 \\
55.869 \\
56.336\end{array}$ & $\begin{array}{c}4.57 \\
.073 \\
25.13 \\
55.607 \\
6.07\end{array}$ & $\begin{array}{r}.573 \\
=4.49 \\
51.334 \\
55.349 \\
=5.614\end{array}$ & $\begin{array}{l}53.70 \\
54.167 \\
54.630 \\
55.093 \\
55.550\end{array}$ & 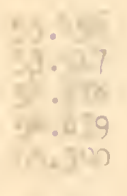 & $\begin{array}{l}53.221 \\
53.570 \\
51.223 \\
54.587 \\
55.246\end{array}$ & $\begin{array}{l}53.968 \\
53.425 \\
53.382 \\
54.338 \\
34.795\end{array}$ & $\begin{array}{l}52.727 \\
53.182 \\
53.636 \\
54.091 \\
54.545\end{array}$ \\
\hline $\begin{array}{l}121 \\
122 \\
12 j \\
124 \\
125\end{array}$ & $\begin{array}{l}57.340 \\
57.327 \\
58.29+6 \\
58.768 \\
59.242\end{array}$ & $\begin{array}{r}7.075 \\
7.547 \\
53.019 \\
58.471 \\
53.952\end{array}$ & $\begin{array}{l}56.011 \\
57.277 \\
37.740 \\
53.216 \\
38.60\end{array}$ & $\begin{array}{l}19.519 \\
37.079 \\
57.477 \\
57.944 \\
9.411\end{array}$ & $\begin{array}{r}0.279 \\
56.744 \\
5.279 \\
7.674 \\
0.140\end{array}$ & $\begin{array}{l}56.017 \\
50.431 \\
56.9^{14} \\
57.477 \\
57.070\end{array}$ & 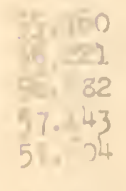 & $\begin{array}{r}5.35 \\
5.453 \\
.422 \\
56.832 \\
57.39\end{array}$ & $\begin{array}{l}55.251 \\
55.728 \\
55.164 \\
55.621 \\
57.078\end{array}$ & $\begin{array}{l}55.7 m \\
55.455 \\
55.909 \\
55.304 \\
56.818\end{array}$ \\
\hline $\begin{array}{l}126 \\
127 \\
120^{\prime} \\
129 \\
130\end{array}$ & $\begin{array}{l}59.716 \\
60.190 \\
60.604 \\
61.137 \\
62.611\end{array}$ & $\begin{array}{l}9.43^{\circ} \\
3.906 \\
0.377 \\
60.347 \\
51.321\end{array}$ & $\begin{array}{l}53.151 \\
57.62 \\
60.091 \\
60.563 \\
61.033\end{array}$ & $\begin{array}{l}0.879 \\
59.340 \\
59.811 \\
63.280 \\
60.746\end{array}$ & $\begin{array}{l}58.075 \\
59.070 \\
59.535 \\
00.777 \\
0.465\end{array}$ & $\begin{array}{l}33.333 \\
50.796 \\
59.259 \\
59.722 \\
60.185\end{array}$ & $\begin{array}{l}38.25 \\
33.25 \\
58.70 \\
39.47 \\
74.78\end{array}$ & $\begin{array}{l}57.793 \\
53.257 \\
53.736 \\
59.274 \\
53.633\end{array}$ & $\begin{array}{l}57.534 \\
57.991 \\
58.447 \\
58.374 \\
59.361\end{array}$ & $\begin{array}{l}57.273 \\
57.727 \\
56.182 \\
58.536 \\
59.091\end{array}$ \\
\hline $\begin{array}{l}131 \\
132 \\
133 \\
134 \\
135\end{array}$ & $\begin{array}{l}62.085 \\
62.559 \\
63.033 \\
63.507 \\
63.981\end{array}$ & $\begin{array}{l}51.792 \\
02.204 \\
62.736 \\
63.228 \\
63.679\end{array}$ & $\begin{array}{l}61.512 \\
61.972 \\
62.441 \\
62.911 \\
63.330\end{array}$ & $\begin{array}{l}61 .=25 \\
51.22 \\
62.153 \\
52.617 \\
63.084\end{array}$ & $\begin{array}{l}0.932 \\
01.395 \\
31.860 \\
22.326 \\
62.791\end{array}$ & $\begin{array}{l}60.648 \\
61.111 \\
61.574 \\
62.037 \\
62.570\end{array}$ & $\begin{array}{l}0.9 \\
-0.29 \\
1.20 \\
5 x \cdot 1=1 \\
-2,12\end{array}$ & $\begin{array}{l}50.092 \\
60.50 \\
51.309 \\
51.468 \\
51.027\end{array}$ & $\begin{array}{l}59.017 \\
50.274 \\
60.731 \\
61.187 \\
51.944\end{array}$ & $\begin{array}{l}59.545 \\
50.320 \\
60.455 \\
60.929 \\
51.304\end{array}$ \\
\hline $\begin{array}{l}135 \\
137 \\
138 \\
139 \\
140\end{array}$ & $\begin{array}{l}64.455 \\
64.329 \\
62.473 \\
05.077 \\
60.351\end{array}$ & $\begin{array}{l}64.151 \\
54.623 \\
65.294 \\
65.560 \\
66.038\end{array}$ & $\begin{array}{l}3.59 \\
64.319 \\
54.763 \\
65.258 \\
65.725\end{array}$ & $\begin{array}{l}3.51 \\
4.019 \\
64.83 \\
64.953 \\
65.421\end{array}$ & $\begin{array}{l}3.256 \\
3.721 \\
4.126 \\
54.651 \\
65.116\end{array}$ & $\begin{array}{l}52.96 \\
63.426 \\
63.30 \\
64.35\end{array}$ & $\begin{array}{r}2.73 \\
=3.24 \\
3.34 \\
-4.25 \\
4.715\end{array}$ & $\begin{array}{r}-3.752 \\
.727\end{array}$ & $\begin{array}{l}62.100 \\
62.557 \\
3.024 \\
63.472 \\
-3.927\end{array}$ & $\begin{array}{l}61.010 \\
62.273 \\
62.727 \\
63.102 \\
-3.636\end{array}$ \\
\hline $\begin{array}{l}141 \\
142 \\
143 \\
144 \\
145\end{array}$ & $\begin{array}{l}65.325 \\
67.297 \\
67.773 \\
68.246 \\
68.720\end{array}$ & $\begin{array}{l}66.509 \\
66.981 \\
67.453 \\
67.925 \\
58.396\end{array}$ & $\begin{array}{l}66.197 \\
66.667 \\
67.136 \\
67.606 \\
63.075\end{array}$ & $\begin{array}{l}55.8=3 \\
60.355 \\
66.22 \\
67.290 \\
67.757\end{array}$ & $\begin{array}{l}5.581 \\
65.247 \\
65.512 \\
65.977 \\
=7.4+2\end{array}$ & $\begin{array}{l}5.278 \\
65.741 \\
66.224 \\
66.657 \\
37.137\end{array}$ & $\begin{array}{r}.77 \\
0.38 \\
.-39 \\
.319 \\
25\end{array}$ & $=6$. & $\begin{array}{r}.34 \\
.247 \\
55.297 \\
5.753 \\
5.217\end{array}$ & $\begin{array}{l}4.291 \\
64.545 \\
65.270 \\
65.455 \\
65.909\end{array}$ \\
\hline $\begin{array}{l}146 \\
147 \\
148 \\
149\end{array}$ & $\begin{array}{l}69.194 \\
69.625 \\
70.142 \\
70.616 \\
71.090\end{array}$ & $\begin{array}{l}68.358 \\
59.340 \\
69.011 \\
70.283 \\
70.755\end{array}$ & $\begin{array}{l}63.545 \\
69.024 \\
69.464 \\
69.953 \\
70.423\end{array}$ & $\begin{array}{l}88.224 \\
8.012 \\
59.153 \\
59.625 \\
70.793\end{array}$ & $\begin{array}{l}7.9) 7 \\
3.372 \\
63.337 \\
09.302 \\
3.77\end{array}$ & $\begin{array}{l}67.533 \\
3.25 \\
63.513 \\
68.93 \\
69.4 .41\end{array}$ & 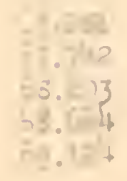 & $\begin{array}{l}67.09 \\
63.25\end{array}$ & $\begin{array}{l}6 . .557 \\
57.707 \\
57.580 \\
68.037 \\
61.493\end{array}$ & $\begin{array}{r}65.364 \\
5.018 \\
67.273 \\
67.727 \\
68.182\end{array}$ \\
\hline
\end{tabular}

\section{LIBRARY STATE PLANT BOARD}




$$
\text { . }
$$




\begin{tabular}{|c|c|c|c|c|c|c|c|c|c|c|}
\hline & 211 & 212 & 213 & 224 & 215 & 216 & 217 & 218 & 219 & 220 \\
\hline $\begin{array}{l}151 \\
152 \\
153 \\
154 \\
155\end{array}$ & $\begin{array}{l}71.584 \\
72.038 \\
72.512 \\
72.986 \\
73.460\end{array}$ & $\begin{array}{l}1.226 \\
11.698 \\
72.170 \\
72.642 \\
73.113\end{array}$ & $\begin{array}{l}70.892 \\
71.362 \\
71.831 \\
72.300 \\
72.770\end{array}$ & $\begin{array}{l}70.561 \\
71.028 \\
71.495 \\
71.963 \\
72.430\end{array}$ & $\begin{array}{l}70.233 \\
70.698 \\
71.163 \\
71.628 \\
72.093\end{array}$ & $\begin{array}{l}69.907 \\
70.370 \\
70.833 \\
11.296 \\
71.759\end{array}$ & $\begin{array}{l}69.585 \\
70.046 \\
70.507 \\
70.958 \\
71.429\end{array}$ & $\begin{array}{l}69.266 \\
69.725 \\
70.183 \\
70.642 \\
71.101\end{array}$ & $\begin{array}{l}68.950 \\
69.4106 \\
69.863 \\
70.320 \\
70.776\end{array}$ & $\begin{array}{l}58.636 \\
69.091 \\
69.545 \\
70.000 \\
70.455\end{array}$ \\
\hline $\begin{array}{l}156 \\
157 \\
158 \\
159 \\
160\end{array}$ & $\begin{array}{l}73.934 \\
74.408 \\
74.882 \\
75.355 \\
75.829\end{array}$ & $\begin{array}{l}73.585 \\
74.057 \\
74.528 \\
75.000 \\
75.472\end{array}$ & $\begin{array}{l}73.239 \\
73.709 \\
74.178 \\
74.648 \\
75.11\end{array}$ & $\begin{array}{l}72.897 \\
73.364 \\
73.832 \\
74.299 \\
74.766\end{array}$ & $\begin{array}{l}72.558 \\
73.023 \\
73.488 \\
73.953 \\
74.419\end{array}$ & $\begin{array}{l}72.222 \\
72.685 \\
73.148 \\
73.611 \\
74.074\end{array}$ & $\begin{array}{l}71.889 \\
72.350 \\
72.811 \\
73.272 \\
73.733\end{array}$ & $\begin{array}{l}71.560 \\
72.018 \\
72.477 \\
72.936 \\
73.394\end{array}$ & $\begin{array}{l}71.233 \\
71.689 \\
72.146 \\
72.603 \\
73.059\end{array}$ & $\begin{array}{l}70.909 \\
11.364 \\
71.818 \\
72.273 \\
72.727\end{array}$ \\
\hline $\begin{array}{l}161 \\
162 \\
163 \\
164 \\
165\end{array}$ & $\begin{array}{l}76.303 \\
76.777 \\
77.251 \\
77.725 \\
78.199\end{array}$ & $\begin{array}{l}75.943 \\
76.415 \\
76.837 \\
77.358 \\
77.830\end{array}$ & $\begin{array}{l}75.587 \\
76.056 \\
76.526 \\
76.995 \\
77.465\end{array}$ & $\begin{array}{l}75.234 \\
75.701 \\
75.168 \\
76.636 \\
77.103\end{array}$ & $\begin{array}{l}74.884 \\
75.349 \\
75.814 \\
76.279 \\
76.744\end{array}$ & $\begin{array}{l}74.537 \\
75.037 \\
75.463 \\
75.926 \\
75.389\end{array}$ & $\begin{array}{l}74.194 \\
74.654 \\
75.115 \\
75.576 \\
76.237\end{array}$ & $\begin{array}{l}73.853 \\
74.312 \\
74.771 \\
75.229 \\
75.683\end{array}$ & $\begin{array}{l}73.516 \\
73.973 \\
74.429 \\
74.886 \\
75.342\end{array}$ & $\begin{array}{l}73.182 \\
73.636 \\
74.091 \\
74.545 \\
75.005\end{array}$ \\
\hline $\begin{array}{l}166 \\
167 \\
168 \\
169 \\
170\end{array}$ & $\begin{array}{l}78.673 \\
79.147 \\
79.621 \\
80.295 \\
80.569\end{array}$ & $\begin{array}{l}78.302 \\
78.774 \\
79.245 \\
79.717 \\
80.189\end{array}$ & $\begin{array}{l}77.934 \\
78.404 \\
78.873 \\
79.343 \\
79.812\end{array}$ & $\begin{array}{l}77.570 \\
78.037 \\
78.505 \\
78.972 \\
79.439\end{array}$ & $\begin{array}{l}77.209 \\
77.674 \\
78.140 \\
78.605 \\
79.070\end{array}$ & $\begin{array}{l}76.852 \\
77.315 \\
77.778 \\
78.241 \\
78.704\end{array}$ & $\begin{array}{l}76.498 \\
76.959 \\
77.419 \\
77.530 \\
73.341\end{array}$ & $\begin{array}{l}76.147 \\
76.606 \\
77.064 \\
77.523 \\
77.932\end{array}$ & $\begin{array}{l}75.799 \\
76.256 \\
75.712 \\
77.159 \\
77.626\end{array}$ & $\begin{array}{l}75.455 \\
75.909 \\
75.364 \\
76.818 \\
77.273\end{array}$ \\
\hline $\begin{array}{l}171 \\
172 \\
173 \\
174 \\
175\end{array}$ & $\begin{array}{l}81.043 \\
81.517 \\
8.991 \\
82.464 \\
82.938\end{array}$ & $\begin{array}{l}30.650 \\
81.132 \\
81.604 \\
82.075 \\
82.547\end{array}$ & $\begin{array}{l}80.282 \\
80.751 \\
81.221 \\
81.690 \\
82.150\end{array}$ & $\begin{array}{l}79.907 \\
80.374 \\
30.841 \\
81.308 \\
81.776\end{array}$ & $\begin{array}{l}79.535 \\
80.050 \\
80.465 \\
80.930 \\
81.395\end{array}$ & $\begin{array}{l}79.167 \\
79.630 \\
80.093 \\
80.556 \\
8.019\end{array}$ & $\begin{array}{l}78.872 \\
79.263 \\
79.724 \\
30.184 \\
50.645\end{array}$ & $\begin{array}{l}78.440 \\
78.899 \\
79.3=8 \\
79.817 \\
80.275\end{array}$ & $\begin{array}{l}78.082 \\
78.539 \\
78.995 \\
79.452 \\
79.909\end{array}$ & $\begin{array}{l}77.727 \\
78.182 \\
78.636 \\
79.091 \\
79.545\end{array}$ \\
\hline $\begin{array}{l}176 \\
177 \\
178 \\
179 \\
180\end{array}$ & $\begin{array}{l}83.412 \\
83.886 \\
84.360 \\
84.834 \\
85.308\end{array}$ & $\begin{array}{l}83.019 \\
83.491 \\
83.962 \\
24.434 \\
34.906\end{array}$ & $\begin{array}{l}22.629 \\
83.099 \\
83.568 \\
84.038 \\
84.507\end{array}$ & $\begin{array}{l}82.243 \\
82.710 \\
83.178 \\
83.645 \\
84.112\end{array}$ & $\begin{array}{l}81.860 \\
82.326 \\
32.791 \\
83.256 \\
83.721\end{array}$ & $\begin{array}{l}81.431 \\
81.944 \\
82.407 \\
82.870 \\
83.333\end{array}$ & $\begin{array}{l}81.176 \\
61.567 \\
32.728 \\
32.488 \\
32.949\end{array}$ & $\begin{array}{l}80.734 \\
81.193 \\
81.551 \\
82.110 \\
82.569\end{array}$ & $\begin{array}{l}80.365 \\
80.822 \\
81.279 \\
81.735 \\
82.192\end{array}$ & $\begin{array}{l}80.250 \\
80.455 \\
80.909 \\
81.364 \\
81.818\end{array}$ \\
\hline $\begin{array}{l}18 \\
182 \\
183 \\
184 \\
185\end{array}$ & $\begin{array}{l}85.782 \\
36.256 \\
86.730 \\
37.204 \\
87.678\end{array}$ & $\begin{array}{l}85.377 \\
85.849 \\
36.321 \\
86.792 \\
87.264\end{array}$ & $\begin{array}{l}84.977 \\
85.446 \\
85.915 \\
36.385 \\
86.854\end{array}$ & $\begin{array}{l}84.579 \\
85.047 \\
85.514 \\
85.981 \\
86.449\end{array}$ & $\begin{array}{l}84.186 \\
84.651 \\
85.116 \\
85.521 \\
86.047\end{array}$ & $\begin{array}{l}83.796 \\
84.259 \\
24.722 \\
35.185 \\
35.648\end{array}$ & $\begin{array}{l}83.410 \\
83.872 \\
84.332 \\
84.793 \\
85.253\end{array}$ & $\begin{array}{l}83.028 \\
83.485 \\
83.945 \\
84.404 \\
84.862\end{array}$ & $\begin{array}{l}82.648 \\
83.105 \\
83.562 \\
84.01 \cdot 8 \\
84.475\end{array}$ & $\begin{array}{l}82.273 \\
82.727 \\
83.182 \\
83.636 \\
84.091\end{array}$ \\
\hline $\begin{array}{l}186 \\
137 \\
188 \\
139 \\
190\end{array}$ & $\begin{array}{l}88.152 \\
88.526 \\
89.100 \\
89.573 \\
90.047\end{array}$ & $\begin{array}{l}87.736 \\
38.208 \\
88.679 \\
39.151 \\
89.623\end{array}$ & $\begin{array}{l}87.324 \\
87.793 \\
88.263 \\
88.732 \\
89.202\end{array}$ & $\begin{array}{l}86.916 \\
87.383 \\
87.850 \\
88.318 \\
38.785\end{array}$ & $\begin{array}{l}86.512 \\
86.977 \\
87.442 \\
87.907 \\
88.372\end{array}$ & $\begin{array}{l}86.111 \\
86.574 \\
87.037 \\
87.503 \\
87.953\end{array}$ & $\begin{array}{l}85.714 \\
86.175 \\
36.636 \\
87.097 \\
87.558\end{array}$ & $\begin{array}{l}85.321 \\
35.780 \\
66.239 \\
36.697 \\
87.156\end{array}$ & $\begin{array}{l}84.932 \\
85.388 \\
85.845 \\
86.301 \\
86.51\end{array}$ & $\begin{array}{l}84.545 \\
85.000 \\
85.455 \\
85.909 \\
85.354\end{array}$ \\
\hline $\begin{array}{l}191 \\
192 \\
193 \\
194 \\
195\end{array}$ & $\begin{array}{l}90.521 \\
90.995 \\
91.469 \\
91.943 \\
92.417\end{array}$ & $\begin{array}{l}90.094 \\
90.566 \\
91.038 \\
91.509 \\
91.981\end{array}$ & $\begin{array}{l}89.671 \\
90.141 \\
90.610 \\
91.030 \\
91.549\end{array}$ & $\begin{array}{l}39.252 \\
39.720 \\
30.187 \\
90.654 \\
91.121\end{array}$ & $\begin{array}{l}88.337 \\
39.302 \\
89.767 \\
90.233 \\
90.598\end{array}$ & $\begin{array}{l}08.426 \\
33.389 \\
89.352 \\
89.815 \\
90.278\end{array}$ & $\begin{array}{l}33.018 \\
38.479 \\
30.945 \\
39.401 \\
39.862\end{array}$ & $\begin{array}{l}87.615 \\
83.073 \\
88.532 \\
83.991 \\
89.451\end{array}$ & $\begin{array}{l}87.215 \\
87.571 \\
88.128 \\
88.584 \\
89.041\end{array}$ & $\begin{array}{l}86.818 \\
87.273 \\
87.727 \\
88.182 \\
88.636\end{array}$ \\
\hline $\begin{array}{l}196 \\
197 \\
198 \\
199 \\
200\end{array}$ & $\begin{array}{l}92.891 \\
93.365 \\
93.839 \\
94.313 \\
94.787\end{array}$ & $\begin{array}{l}92.453 \\
92.925 \\
93.395 \\
93.868 \\
94.310\end{array}$ & $\begin{array}{l}92.019 \\
92.488 \\
92.958 \\
93.427 \\
93.897\end{array}$ & $\begin{array}{l}91.539 \\
92.056 \\
92.523 \\
92.991 \\
93.458\end{array}$ & $\begin{array}{l}91.163 \\
91.528 \\
92.093 \\
92.558 \\
93.023\end{array}$ & $\begin{array}{l}90.741 \\
91.204 \\
91.657 \\
92.130 \\
92.593\end{array}$ & $\begin{array}{l}90.323 \\
90.733 \\
91.244 \\
91.705 \\
92.166\end{array}$ & $\begin{array}{l}89.908 \\
90.367 \\
90.326 \\
91.284 \\
91.743\end{array}$ & $\begin{array}{l}89.498 \\
89.954 \\
90.411 \\
90.868 \\
91.324\end{array}$ & $\begin{array}{l}39.091 \\
89.545 \\
90.000 \\
90.455 \\
90.909\end{array}$ \\
\hline
\end{tabular}





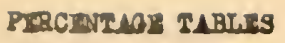

$\begin{array}{lllllllll}21 & 212 & 213 & 214 & 215 & 216 & 217 & 218 & 229\end{array}$

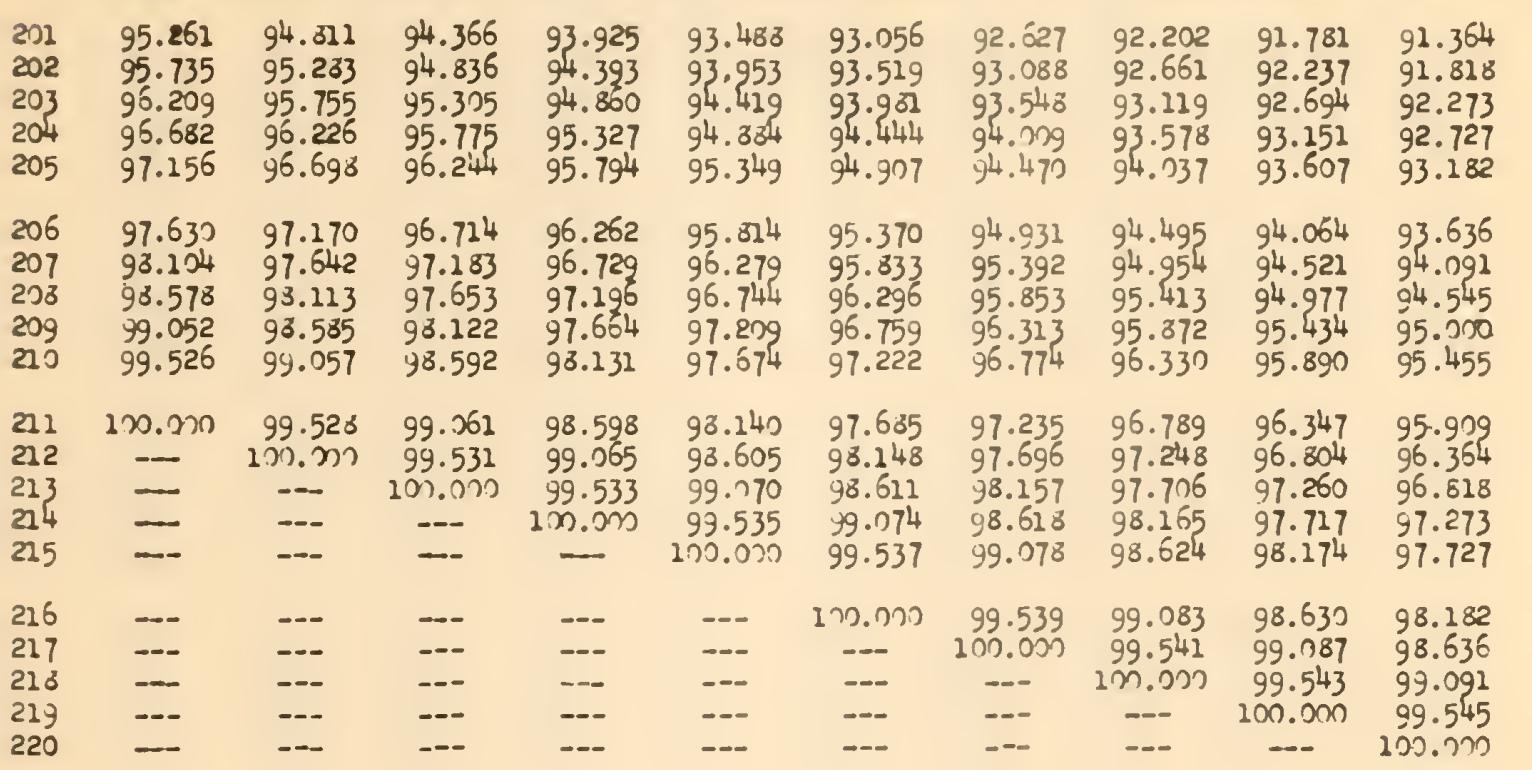





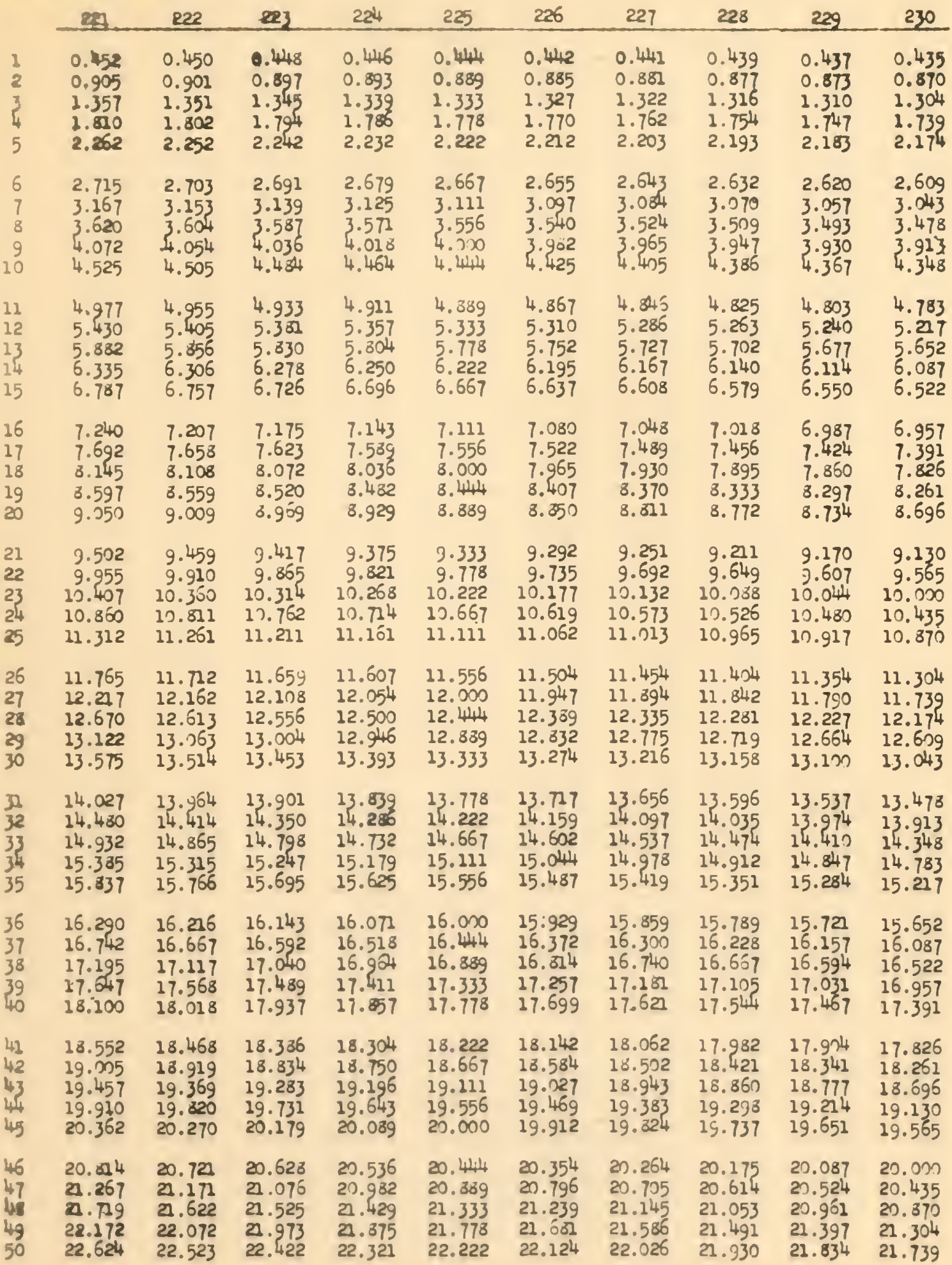





\begin{tabular}{llllllllll}
221 & 222 & 223 & 224 & 225 & 226 & 227 & 228 & 229 & 230 \\
\hline
\end{tabular}

23.07722 .973

2.539

23.922

23.423

24.434

24. 887

23. 874

24. 324

24.775

25.339

25. 792

20.244

26.697

25.225

25.676

26.126

27.149

26.577

27.027

27.602

28.254

23.507

28.359

29.412

27.477

27.928

20.378

26.829

29.279

29.364

32.317

29.133

30.130

32.631

30.709

31.222

31.081

31.532

32.127

32.579

31.982

33.1732

33.434

32.432

32.383

33.333

33.937

33.784

$34.38 y$

34.242

34.234

35.294

34.685

35.135

35.747

35.536

35.036

80

81

82

83

84

35

6

37

88

89

90

91

92

93

95

gó

97

y3

99

100

36.652

35.485

37.124

37.557

$33.0 \%$

36.937

37.387

37.838

30.914

39.357

39.819

40.271

40.724

38. 739

39.189

39.640

40.090

40. 541

41.176

41.629

42.081

42.534

42.985

40.991

42.793

43.439

43.091

44.796

43.243

43.694

44.144

$+5.249$

44.595

45.045
$22.870 \quad 22.768$

23.318

23.767

24.215

24.634

25.112

25.561

25.009

25.457

26.906

27.354

27.803

28.251

28.793

29.148

29.596

30.045

30.493

30.942

31.390

31.839

32.287

32.735

33.184

33.632

34.031

34.529

34.978

35.426

35.874

36.323

36.77

37.220

37.668

38.117

38.565

39.013

39.462

39.910

40.359

40.377

41.256

$41.892 \quad 41.734$

$42.342 \quad 42.152$

$+2.601$

43.049

23.214

23.661

24.107

24.554

25.000

25.446

25.893

26.339

26.736

27.232

27.679

28.125

23.57

29.018

29.464

29.911

30.357

30.804

31.250

31.696

32.143

32.589

33.036

33.482

33.929

34.375

34.821

35.268

35.714

35.161

36.607

37.054

37.500

37.946

38.393

38.339

39.285

39.732

40.179

40.625

41.072

41.518

41.924

42.411

43.493

43.946
4.395

4.343

$22.667 \quad 22.566$

23. 111

23.556

24.200

24. 444

23.009

23.451

23.894

24.336

24.889

25.333

25.778

26.222

26.667

24.779

25.221

25.664

26.106

26.549

27.111

27.556

23.0n

28.44

26.991

27.434

27.876

20.319

26.889

28.751

29.333

29.204

29.778

30.222

30.667

$2 y .046$

30.086

30.531

31.111

32.973

31.556

32.000

32.444

32.339

31.416

31.85

32.301

32.743

$33.333 \quad 33.186$

33.778

34.222

33.628

34.557

35.111

35.556

34.071

34.513

34.956

35.398

36. 270

35.841

$30.444 \quad 36.283$

$36.889 \quad 36.726$

$37.333 \quad 37.168$

37.778

37.511

36.222

36.053

38.567

38.496

39.111

39.556

38.938

39.331

39.823

40.444

4). 839

40. 255

41.333

41.778 


\begin{tabular}{|c|c|c|c|c|c|c|c|c|c|c|}
\hline & 82 & 222 & 223 & 224 & $\infty 3$ & 226 & 227 & 228 & 229 & 230 \\
\hline $\begin{array}{l}101 \\
102 \\
103 \\
104 \\
105\end{array}$ & $\begin{array}{l}45.701 \\
46.154 \\
46.606 \\
47.059 \\
47.511\end{array}$ & $\begin{array}{l}45.495 \\
45.346 \\
46.396 \\
46.847 \\
47.297\end{array}$ & $\begin{array}{l}45.291 \\
45.740 \\
46.288 \\
46.637 \\
47.085\end{array}$ & $\begin{array}{l}45.089 \\
45.536 \\
45.982 \\
46.429 \\
46.875\end{array}$ & $\begin{array}{l}44.889 \\
45.333 \\
45.778 \\
48.222 \\
46.667\end{array}$ & $\begin{array}{l}44.690 \\
45.133 \\
45.575 \\
46.018 \\
46.460\end{array}$ & $\begin{array}{l}44.493 \\
44.934 \\
45.374 \\
45.825 \\
46.256\end{array}$ & $\begin{array}{l}44.298 \\
44.737 \\
45.175 \\
45.614 \\
45.053\end{array}$ & $\begin{array}{l}44.105 \\
44.541 \\
44.978 \\
45.415 \\
45.852\end{array}$ & $\begin{array}{l}43.913 \\
44.348 \\
44.783 \\
45.217 \\
45.652\end{array}$ \\
\hline $\begin{array}{l}106 \\
107 \\
108 \\
109 \\
110\end{array}$ & $\begin{array}{l}47.964 \\
48.416 \\
48.869 \\
49.321 \\
49.774\end{array}$ & $\begin{array}{l}47.748 \\
43.198 \\
48.649 \\
49.099 \\
49.550\end{array}$ & $\begin{array}{l}47.534 \\
47.982 \\
48.430 \\
48.879 \\
49.327\end{array}$ & $\begin{array}{l}47.321 \\
47.768 \\
43.214 \\
48.661 \\
49.107\end{array}$ & $\begin{array}{l}47.111 \\
47.556 \\
48.000 \\
48.444 \\
48.839\end{array}$ & $\begin{array}{l}46.903 \\
47.345 \\
47.788 \\
48.230 \\
48.673\end{array}$ & & $\begin{array}{l}45.491 \\
45.930 \\
47.358 \\
47.807 \\
48.246\end{array}$ & $\begin{array}{l}46.288 \\
45.725 \\
47.162 \\
47.598 \\
48.035\end{array}$ & $\begin{array}{l}46.087 \\
46.522 \\
46.957 \\
47.391 \\
47.826\end{array}$ \\
\hline $\begin{array}{l}111 \\
112 \\
113 \\
114 \\
115\end{array}$ & $\begin{array}{l}50.226 \\
50.679 \\
51.131 \\
51.534 \\
52.036\end{array}$ & $\begin{array}{l}50.000 \\
50.450 \\
50.901 \\
51.351 \\
51.802\end{array}$ & $\begin{array}{l}49.776 \\
50.224 \\
50.673 \\
51.121 \\
51.570\end{array}$ & $\begin{array}{l}49.5513 \\
50.000 \\
50.446 \\
50.893 \\
51.339\end{array}$ & $\begin{array}{l}49.333 \\
49.778 \\
50.222 \\
20.657 \\
51.111\end{array}$ & $\begin{array}{l}49.115 \\
49.558 \\
50.050 \\
50.442 \\
50.835\end{array}$ & $\begin{array}{l}48.899 \\
49.339 \\
49.780 \\
50.220 \\
50.661\end{array}$ & $\begin{array}{l}48.684 \\
49.123 \\
49.561 \\
50.000 \\
50.439\end{array}$ & $\begin{array}{l}48.472 \\
48.908 \\
49.345 \\
49.782 \\
50.218\end{array}$ & $\begin{array}{l}48.261 \\
48.696 \\
49.130 \\
49.565 \\
50.000\end{array}$ \\
\hline $\begin{array}{l}116 \\
117 \\
118 \\
119 \\
120\end{array}$ & $\begin{array}{l}52.489 \\
52.941 \\
53.394 \\
53.646 \\
54.299\end{array}$ & $\begin{array}{l}52.252 \\
52.703 \\
53.153 \\
53.604 \\
54.054\end{array}$ & $\begin{array}{l}52.018 \\
52.466 \\
52.915 \\
53.363 \\
53.312\end{array}$ & $\begin{array}{l}51.725 \\
52.232 \\
52.679 \\
53.125 \\
53.571\end{array}$ & $\begin{array}{l}31.556 \\
52.030 \\
52.444 \\
52.839 \\
53.333\end{array}$ & $\begin{array}{l}51.327 \\
51.770 \\
52.212 \\
52.655 \\
53.097\end{array}$ & $\begin{array}{l}51.101 \\
51.542 \\
51.982 \\
52.423 \\
52.863\end{array}$ & $\begin{array}{l}50.877 \\
51.316 \\
51.754 \\
52.193 \\
52.632\end{array}$ & $\begin{array}{l}50.655 \\
53.092 \\
51.528 \\
51.965 \\
52.402\end{array}$ & $\begin{array}{l}50.435 \\
50.870 \\
51.304 \\
51.739 \\
52.174\end{array}$ \\
\hline $\begin{array}{l}121 \\
122 \\
123 \\
124 \\
125\end{array}$ & $\begin{array}{l}54.751 \\
55.204 \\
55.656 \\
56.109 \\
56.561\end{array}$ & $\begin{array}{l}54.505 \\
54.955 \\
55.405 \\
55.856 \\
56.306\end{array}$ & $\begin{array}{l}54.260 \\
54.709 \\
55.157 \\
55.605 \\
56.354\end{array}$ & $\begin{array}{l}54.018 \\
54.464 \\
54.921 \\
55.357 \\
55.304\end{array}$ & $\begin{array}{l}53.778 \\
54.222 \\
54.667 \\
55.111 \\
55.556\end{array}$ & $\begin{array}{l}53.540 \\
53.982 \\
54.425 \\
54.867 \\
55.310\end{array}$ & $\begin{array}{l}53.304 \\
53.744 \\
54.183 \\
54.626 \\
55.066\end{array}$ & $\begin{array}{l}53.070 \\
53.509 \\
53.947 \\
54.386 \\
54.825\end{array}$ & $\begin{array}{l}52.838 \\
53.275 \\
53.722 \\
54.148 \\
54.585\end{array}$ & $\begin{array}{l}52.609 \\
53.043 \\
53.478 \\
53.913 \\
54.348\end{array}$ \\
\hline $\begin{array}{l}126 \\
127 \\
128 \\
129 \\
130\end{array}$ & $\begin{array}{l}57.014 \\
57.466 \\
57.919 \\
58.37 \\
58.824\end{array}$ & $\begin{array}{l}56.757 \\
57.207 \\
57.658 \\
58.108 \\
53.559\end{array}$ & $\begin{array}{l}56.502 \\
56.951 \\
57.399 \\
57.348 \\
53.296\end{array}$ & $\begin{array}{l}56.250 \\
56.696 \\
57.143 \\
57.589 \\
53.036\end{array}$ & $\begin{array}{l}56.390 \\
56.44 \\
56.839 \\
57.333 \\
57.778\end{array}$ & $\begin{array}{l}55.752 \\
56.195 \\
56.637 \\
57.080 \\
57.522\end{array}$ & $\begin{array}{l}55.507 \\
55.947 \\
56.388 \\
56.828 \\
57.269\end{array}$ & $\begin{array}{l}55.263 \\
55.702 \\
56.140 \\
56.579 \\
57.118\end{array}$ & $\begin{array}{l}55.022 \\
55.459 \\
55.895 \\
56.332 \\
56.769\end{array}$ & $\begin{array}{l}54.783 \\
55.217 \\
55.652 \\
56.087 \\
56.522\end{array}$ \\
\hline $\begin{array}{l}131 \\
132 \\
133 \\
134 \\
335\end{array}$ & $\begin{array}{l}59.276 \\
59.729 \\
60.181 \\
60.633 \\
61.086\end{array}$ & $\begin{array}{l}59.419 \\
59.459 \\
59.910 \\
60.360 \\
67.811\end{array}$ & $\begin{array}{l}58.744 \\
59.293 \\
59.642 \\
60.093 \\
60.533\end{array}$ & $\begin{array}{l}53.422 \\
53.929 \\
59.375 \\
59.321 \\
63.268\end{array}$ & $\begin{array}{l}58.222 \\
58.667 \\
59.111 \\
59.556 \\
60.000\end{array}$ & $\begin{array}{l}57.965 \\
58.407 \\
58.850 \\
59.292 \\
59.735\end{array}$ & & $\begin{array}{l}57.456 \\
57.895 \\
58.333 \\
58.772 \\
59.211\end{array}$ & $\begin{array}{l}57.205 \\
57.642 \\
58.079 \\
58.515 \\
58.952\end{array}$ & $\begin{array}{l}56.957 \\
57.391 \\
57.826 \\
58.261 \\
58.696\end{array}$ \\
\hline $\begin{array}{l}136 \\
137 \\
133 \\
139 \\
145\end{array}$ & $\begin{array}{l}61.538 \\
61.991 \\
62.443 \\
62.396 \\
63.348\end{array}$ & $\begin{array}{l}61.261 \\
61.712 \\
62.162 \\
62.613 \\
63.063\end{array}$ & $\begin{array}{l}60.987 \\
61.435 \\
61.333 \\
62.332 \\
62.780\end{array}$ & $\begin{array}{l}60.724 \\
61.161 \\
61.607 \\
62.054 \\
62.500\end{array}$ & $\begin{array}{l}60.444 \\
60.889 \\
61.333 \\
61.773 \\
62.222\end{array}$ & $\begin{array}{l}60.177 \\
60.619 \\
61.062 \\
61.504 \\
61.947\end{array}$ & $\begin{array}{l}59.912 \\
60.352 \\
60.793 \\
61.233 \\
61.674\end{array}$ & $\begin{array}{l}59.649 \\
60.088 \\
60.526 \\
60.965 \\
61.404\end{array}$ & $\begin{array}{l}59.339 \\
59.825 \\
60.252 \\
60.699 \\
61.135\end{array}$ & $\begin{array}{l}59.130 \\
59.565 \\
60.230 \\
60.435 \\
60.870\end{array}$ \\
\hline $\begin{array}{l}141 \\
142 \\
143 \\
144 \\
145\end{array}$ & $\begin{array}{l}63.801 \\
64.253 \\
64.706 \\
65.158 \\
65.611\end{array}$ & $\begin{array}{l}63.514 \\
63.964 \\
64.414 \\
64.865 \\
65.315\end{array}$ & $\begin{array}{l}63.229 \\
63.677 \\
44.126 \\
64.574 \\
65.222\end{array}$ & $\begin{array}{l}52.246 \\
63.393 \\
63.339 \\
54.235 \\
64.732\end{array}$ & $\begin{array}{l}62.537 \\
63.111 \\
63.555 \\
64.327 \\
54.441\end{array}$ & $\begin{array}{l}62.339 \\
62.832 \\
63.274 \\
63.717 \\
64.159\end{array}$ & $\begin{array}{l}62.115 \\
62.555 \\
62.996 \\
63.436 \\
53.377\end{array}$ & $\begin{array}{l}61.812 \\
62.281 \\
62.719 \\
53.158 \\
63.596\end{array}$ & $\begin{array}{l}61.572 \\
62.029 \\
62.445 \\
62.882 \\
63.319\end{array}$ & $\begin{array}{l}61.304 \\
61.739 \\
62.174 \\
62.609 \\
63.043\end{array}$ \\
\hline $\begin{array}{l}146 \\
147 \\
148 \\
149 \\
150\end{array}$ & $\begin{array}{l}66.063 \\
66.516 \\
66.968 \\
67.421 \\
6 \% .873\end{array}$ & $\begin{array}{l}65.766 \\
65.216 \\
66.657 \\
67.117 \\
67.558\end{array}$ & $\begin{array}{l}66.366 \\
66.316 \\
67.265\end{array}$ & $\begin{array}{l}65.179 \\
55.625 \\
60.071 \\
65.513 \\
65.904\end{array}$ & $\begin{array}{l}64.333 \\
55.333 \\
65.778 \\
50.222 \\
50.057\end{array}$ & $\begin{array}{l}24.602 \\
65.044 \\
65.437 \\
55.929 \\
65.372\end{array}$ & $\begin{array}{l}64.758 \\
65.198 \\
65.639 \\
66.779\end{array}$ & $\begin{array}{l}64.035 \\
64.474 \\
64.912 \\
65.351 \\
65.789\end{array}$ & $\begin{array}{l}63.755 \\
44.192 \\
44.629 \\
65.066 \\
65.502\end{array}$ & $\begin{array}{l}63.478 \\
63.913 \\
64.348 \\
64.783 \\
65.217\end{array}$ \\
\hline
\end{tabular}




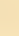




\begin{tabular}{|c|c|c|c|c|c|c|c|c|c|c|}
\hline & 221 & 222 & 223 & 224 & 225 & 226 & 227 & 228 & 229 & 230 \\
\hline $\begin{array}{l}152 \\
152 \\
153 \\
154 \\
155\end{array}$ & $\begin{array}{l}68.326 \\
68.778 \\
69.231 \\
69.683 \\
70.136\end{array}$ & $\begin{array}{l}68.018 \\
68.468 \\
68.919 \\
69.369 \\
69.820\end{array}$ & $\begin{array}{l}67.713 \\
68.161 \\
68.619 \\
69.058 \\
69.507\end{array}$ & $\begin{array}{l}67.411 \\
67.857 \\
68.304 \\
68.750 \\
69.196\end{array}$ & $\begin{array}{l}67.111 \\
67.556 \\
68.000 \\
68.444 \\
68.889\end{array}$ & $\begin{array}{l}66.814 \\
67.257 \\
67.699 \\
68.142 \\
68.584\end{array}$ & $\begin{array}{l}66.520 \\
66.960 \\
67.4 n 1 \\
67.841 \\
68.282\end{array}$ & $\begin{array}{l}66.228 \\
66.667 \\
67.155 \\
67.54 \sqrt{4} \\
67.982\end{array}$ & $\begin{array}{l}65.939 \\
66.376 \\
66.812 \\
67.249 \\
67.686\end{array}$ & $\begin{array}{l}65.652 \\
66.087 \\
66.522 \\
66.957 \\
67.391\end{array}$ \\
\hline $\begin{array}{l}156 \\
157 \\
258 \\
159 \\
160\end{array}$ & $\begin{array}{l}70.588 \\
7.041 \\
7.493 \\
7.946 \\
72.398\end{array}$ & $\begin{array}{l}70.270 \\
70.721 \\
71.171 \\
71.622 \\
72.072\end{array}$ & $\begin{array}{l}69.955 \\
70.404 \\
70.852 \\
71.300 \\
71.749\end{array}$ & $\begin{array}{l}69.043 \\
70.089 \\
70.536 \\
70.982 \\
71.429\end{array}$ & $\begin{array}{l}69.333 \\
69.778 \\
70.222 \\
70.657 \\
71.111\end{array}$ & $\begin{array}{l}69.027 \\
69.469 \\
69.912 \\
70.354 \\
70.796\end{array}$ & $\begin{array}{l}68.722 \\
69.163 \\
69.604 \\
70.044 \\
70.485\end{array}$ & $\begin{array}{l}68.421 \\
68.860 \\
69.298 \\
69.737 \\
70.175\end{array}$ & $\begin{array}{l}68.122 \\
68.559 \\
68.996 \\
69.432 \\
69.869\end{array}$ & $\begin{array}{l}67.826 \\
68.261 \\
68.696 \\
69.130 \\
69.565\end{array}$ \\
\hline $\begin{array}{l}161 \\
162 \\
163 \\
164 \\
165\end{array}$ & $\begin{array}{l}72.351 \\
73.303 \\
73.756 \\
74.208 \\
74.661\end{array}$ & $\begin{array}{l}72.523 \\
72.973 \\
73.423 \\
73.874 \\
74.324\end{array}$ & $\begin{array}{l}72.197 \\
72.446 \\
73.094 \\
73.543 \\
73.991\end{array}$ & $\begin{array}{l}7.875 \\
72.321 \\
72.768 \\
73.214 \\
73.661\end{array}$ & $\begin{array}{l}71.556 \\
72.000 \\
72.444 \\
72.839 \\
73.333\end{array}$ & $\begin{array}{l}7.239 \\
7.681 \\
72.124 \\
72.566 \\
73.009\end{array}$ & $\begin{array}{l}70.925 \\
71.356 \\
71.806 \\
72.247 \\
72.687\end{array}$ & $\begin{array}{l}70.614 \\
7.053 \\
71.491 \\
71.930 \\
72.368\end{array}$ & $\begin{array}{l}70.306 \\
70.742 \\
7.179 \\
7.616 \\
72.052\end{array}$ & $\begin{array}{l}70.000 \\
70.435 \\
70.870 \\
71.304 \\
71.739\end{array}$ \\
\hline $\begin{array}{l}166 \\
167 \\
168 \\
169 \\
170\end{array}$ & $\begin{array}{l}75.113 \\
75.566 \\
76.018 \\
76.471 \\
76.923\end{array}$ & $\begin{array}{l}74.775 \\
75.225 \\
75.676 \\
76.126 \\
76.577\end{array}$ & $\begin{array}{l}74.439 \\
74.888 \\
75.336 \\
75.785 \\
76.233\end{array}$ & $\begin{array}{l}74.107 \\
74.554 \\
75.000 \\
75.446 \\
75.893\end{array}$ & $\begin{array}{l}73.778 \\
74.222 \\
74.667 \\
75.111 \\
75.556\end{array}$ & $\begin{array}{l}73.451 \\
73.894 \\
74.336 \\
74.779 \\
75.221\end{array}$ & $\begin{array}{l}73.128 \\
73.568 \\
74.009 \\
74.449 \\
74.890\end{array}$ & $\begin{array}{l}72.807 \\
73.246 \\
73.684 \\
74.123 \\
74.561\end{array}$ & $\begin{array}{l}72.489 \\
70.926 \\
73.362 \\
73.799 \\
74.236\end{array}$ & $\begin{array}{l}72.174 \\
72.609 \\
73.043 \\
73.478 \\
73.913\end{array}$ \\
\hline $\begin{array}{l}171 \\
172 \\
173 \\
174 \\
175\end{array}$ & $\begin{array}{l}77.376 \\
77.828 \\
78.281 \\
78.733 \\
79.185\end{array}$ & $\begin{array}{l}77.027 \\
77.477 \\
77.928 \\
78.378 \\
78.829\end{array}$ & $\begin{array}{l}76.682 \\
77.130 \\
77.578 \\
78.027 \\
78.475\end{array}$ & $\begin{array}{l}76.339 \\
76.786 \\
77.232 \\
77.679 \\
78.125\end{array}$ & $\begin{array}{l}76.000 \\
76.444 \\
76.889 \\
77.333 \\
77.778\end{array}$ & $\begin{array}{l}75.664 \\
76.106 \\
76.549 \\
76.991 \\
77.434\end{array}$ & $\begin{array}{l}75.330 \\
75.771 \\
76.211 \\
76.652 \\
77.093\end{array}$ & $\begin{array}{l}75.000 \\
75.439 \\
75.877 \\
76 . j 16 \\
76.754\end{array}$ & $\begin{array}{l}74.672 \\
75.109 \\
75.546 \\
75.983 \\
76.419\end{array}$ & $\begin{array}{l}74.348 \\
74.783 \\
75.217 \\
75.652 \\
76.087\end{array}$ \\
\hline $\begin{array}{l}176 \\
177 \\
178 \\
173 \\
180\end{array}$ & $\begin{array}{l}79.638 \\
80.090 \\
80.543 \\
80.995 \\
81.448\end{array}$ & $\begin{array}{l}79.279 \\
79.730 \\
80.180 \\
80.631 \\
81.031\end{array}$ & $\begin{array}{l}75.924 \\
79.372 \\
79.821 \\
80.269 \\
80.717\end{array}$ & $\begin{array}{l}78.57 \\
79.018 \\
79.464 \\
79.941 \\
80.357\end{array}$ & $\begin{array}{l}78.222 \\
78.667 \\
79.111 \\
79.556 \\
80.000\end{array}$ & $\begin{array}{l}77.876 \\
78.319 \\
78.761 \\
79.204 \\
79.646\end{array}$ & $\begin{array}{l}77.533 \\
77.974 \\
78.414 \\
78.855 \\
79.295\end{array}$ & $\begin{array}{l}77.193 \\
77.632 \\
78.070 \\
78.509 \\
78.947\end{array}$ & $\begin{array}{l}76.356 \\
77.293 \\
77.729 \\
78.166 \\
78.603\end{array}$ & $\begin{array}{l}76.522 \\
76.957 \\
77.391 \\
77.826 \\
78.261\end{array}$ \\
\hline $\begin{array}{l}182 \\
182 \\
183 \\
184 \\
185\end{array}$ & $\begin{array}{l}81.900 \\
82.353 \\
82.805 \\
83.258 \\
83.710\end{array}$ & $\begin{array}{l}81.532 \\
81.982 \\
82.432 \\
82.883 \\
83.333\end{array}$ & $\begin{array}{l}81.166 \\
81.614 \\
82.063 \\
82.511 \\
82.960\end{array}$ & $\begin{array}{l}80.804 \\
81.250 \\
81.696 \\
82.143 \\
82.589\end{array}$ & $\begin{array}{l}80.444 \\
80.839 \\
80.333 \\
81.778 \\
82.222\end{array}$ & $\begin{array}{l}80.088 \\
80.531 \\
80.973 \\
81.416 \\
8.858\end{array}$ & $\begin{array}{l}79.736 \\
80.176 \\
80.617 \\
81.057 \\
81.498\end{array}$ & $\begin{array}{l}79.386 \\
79.825 \\
80.263 \\
80.702 \\
81.140\end{array}$ & $\begin{array}{l}79.039 \\
79.476 \\
79.913 \\
80.349 \\
80.786\end{array}$ & $\begin{array}{l}78.696 \\
79.130 \\
79.565 \\
80.000 \\
80.435\end{array}$ \\
\hline $\begin{array}{l}186 \\
187 \\
188 \\
189 \\
190\end{array}$ & $\begin{array}{l}84.163 \\
84.615 \\
85.068 \\
55.520 \\
85.973\end{array}$ & $\begin{array}{l}83.734 \\
84.234 \\
84.685 \\
85.135 \\
85.586\end{array}$ & $\begin{array}{l}83.408 \\
83.857 \\
84.305 \\
84.753 \\
85.202\end{array}$ & $\begin{array}{l}83.036 \\
83.482 \\
83.929 \\
84.375 \\
84.821\end{array}$ & $\begin{array}{l}82.667 \\
83.111 \\
83.556 \\
84.000 \\
84.4444\end{array}$ & $\begin{array}{l}82.301 \\
82.743 \\
83.186 \\
83.628 \\
84.071\end{array}$ & $\begin{array}{l}81.938 \\
82.379 \\
82.819 \\
83.260 \\
83.700\end{array}$ & $\begin{array}{l}81.579 \\
82.018 \\
82.456 \\
82.895 \\
83.333\end{array}$ & $\begin{array}{l}81.223 \\
81.659 \\
82.096 \\
82.533 \\
82.969\end{array}$ & $\begin{array}{l}80.870 \\
81.304 \\
81.739 \\
82.174 \\
82.609\end{array}$ \\
\hline $\begin{array}{l}191 \\
192 \\
193 \\
194 \\
195\end{array}$ & $\begin{array}{l}86.425 \\
85.878 \\
87.330 \\
87.783 \\
88.235\end{array}$ & $\begin{array}{l}86.036 \\
86.486 \\
86.937 \\
87.387 \\
37.838\end{array}$ & $\begin{array}{l}85.650 \\
86.099 \\
86.547 \\
86.996 \\
87.444\end{array}$ & $\begin{array}{l}85.268 \\
35.714 \\
80.161 \\
85.507 \\
87.054\end{array}$ & $\begin{array}{l}84.839 \\
85.333 \\
85.778 \\
86.222 \\
35.567\end{array}$ & $\begin{array}{l}84.513 \\
24.956 \\
85.398 \\
85.841 \\
86.283\end{array}$ & $\begin{array}{l}84.141 \\
84.581 \\
85.022 \\
85.463 \\
85.903\end{array}$ & $\begin{array}{l}83.772 \\
84.211 \\
84.649 \\
85.088 \\
53.526\end{array}$ & $\begin{array}{l}83.406 \\
83.843 \\
84.279 \\
84.716 \\
85.153\end{array}$ & $\begin{array}{l}83.043 \\
83.478 \\
83.913 \\
84.348 \\
84.783\end{array}$ \\
\hline $\begin{array}{l}196 \\
197 \\
198 \\
199 \\
200\end{array}$ & $\begin{array}{l}88.688 \\
89.140 \\
89.593 \\
90.045 \\
90.498\end{array}$ & $\begin{array}{l}88.238 \\
83.739 \\
89.189 \\
89.640 \\
90.090\end{array}$ & $\begin{array}{l}87.892 \\
38.341 \\
38.789 \\
89.238 \\
89.686\end{array}$ & $\begin{array}{l}87.520 \\
87.946 \\
38.393 \\
83.839 \\
89.286\end{array}$ & $\begin{array}{l}87.111 \\
87.556 \\
83.000 \\
88.414 \\
88.889\end{array}$ & $\begin{array}{l}36.726 \\
87.158 \\
87.611 \\
83.053 \\
88.496\end{array}$ & $\begin{array}{l}86.344 \\
86.784 \\
87.225 \\
37.655 \\
83.106\end{array}$ & $\begin{array}{l}85.965 \\
86.404 \\
85.842 \\
87.231 \\
87.719\end{array}$ & $\begin{array}{l}85.590 \\
86.026 \\
86.463 \\
86.900 \\
87.336\end{array}$ & $\begin{array}{l}85.217 \\
85.652 \\
86.037 \\
86.522 \\
86.957\end{array}$ \\
\hline
\end{tabular}




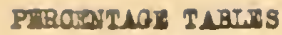

22

90.95090 .541

91.403

91.855

92.308

92760

90.991

91.41

91.892

92.342

93.223
93.665
94.118
94.570

9c. 793

93.243

93.694

94.144

95.023

94.595

95.475

95.928

96.380

36.833

97.285

95.045

95.495

95.946

96.396

96.847

97.738

97.297

$98.190 \quad 97.748$

98.643

99.095

38.198

98.649

99.548

99.299

$100.000 \quad 99.550$

\section{2}

22

22

226

228

229
223

224

225

236

227

228

229 230

\section{$90.135 \quad 89.732$}

90.179

91.03190 .625

$91.480 \quad 91.071$

91.92891 .518

$92.377 \quad 91.964$

92.825
93.274

93.722

9.170

92.411

92.87

93.304

93.750

94.619 94.196

95.06794 .643

$95.516 \quad 95.089$

$95.964 \cdot 95.536$

$96.413 \quad 95.982$

96.861

97.309

96.429

97.758

97.321

$93.206 \quad 97.768$

98.655

98.224

89.333

89.778

90.222

90.667

91.111

88.938

89.381

89.823

90.265

90.708

91. 556

92.000

92.444

92.889

91.150

91.593

92.035

92.478

$93.333 \quad 92.920$

$93.773 \quad 93.363$

94.222

94.667

95.111

95.556

93.805

94.248

94.690

95.133

$96.200 \quad 95.575$

$96.444 \quad 96.013$

$96.889 \quad 96.450$

$97.333 \quad 96.303$

97.778

97.345

$99.103 \quad 98.661$

$49.552 \quad 99.107$

100.250

99.554

-. -

--.

100.000

$93.222 \quad 97.788$

98.65793 .230

99.11198 .673

$99.556 \quad 99.115$

120.00

99.558

-. $\quad 120.020$

$-$

$\begin{array}{rrr}10.020 & 99.559 & 99.123 \\ \ldots & 100.200 & 99.561\end{array}$

-.- 100.000

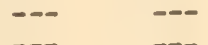

$\cdots$

$\ldots$

$-$

88.158

88.596

89.035

89.474

89.912

90.74990 .351

$91.189 \quad 90.789$

$91.630 \quad 91.228$

$92.070 \quad 91.667$

$92.511 \quad 92.105$

$92.952 \quad 92.544$

$93.392 \quad 92.982$

93.83393 .421

$94.273 \quad 93.860$

94.73494 .298

$95.154 \quad 94.737$

$95.595 \quad 95.175$

$96.035 \quad 95.614$

96.476

96.916

96.053

96.491

$97.357 \quad 96.930$

97.797

98.233

98.678

99.119

$97.3^{\mathrm{h} g}$

97.827

98.246

98.684

87.773

88.210

88.646

89.083

89.520

89.956

90.393

90.830

91.266

91.703

92.140

92.576

93.013

93.450

93.886

94.323

94.760

95.197

95.633

96.070

96.507

96.943

97.380

$97.817 \quad 97.391$

98.25397 .826

$98.690 \quad 98.261$

$99.127 \quad 98.696$

$99.563 \quad 99.130$

... $\quad 100.000 \quad 99.565$

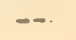

$-\infty$

100.020 

PGR Carr

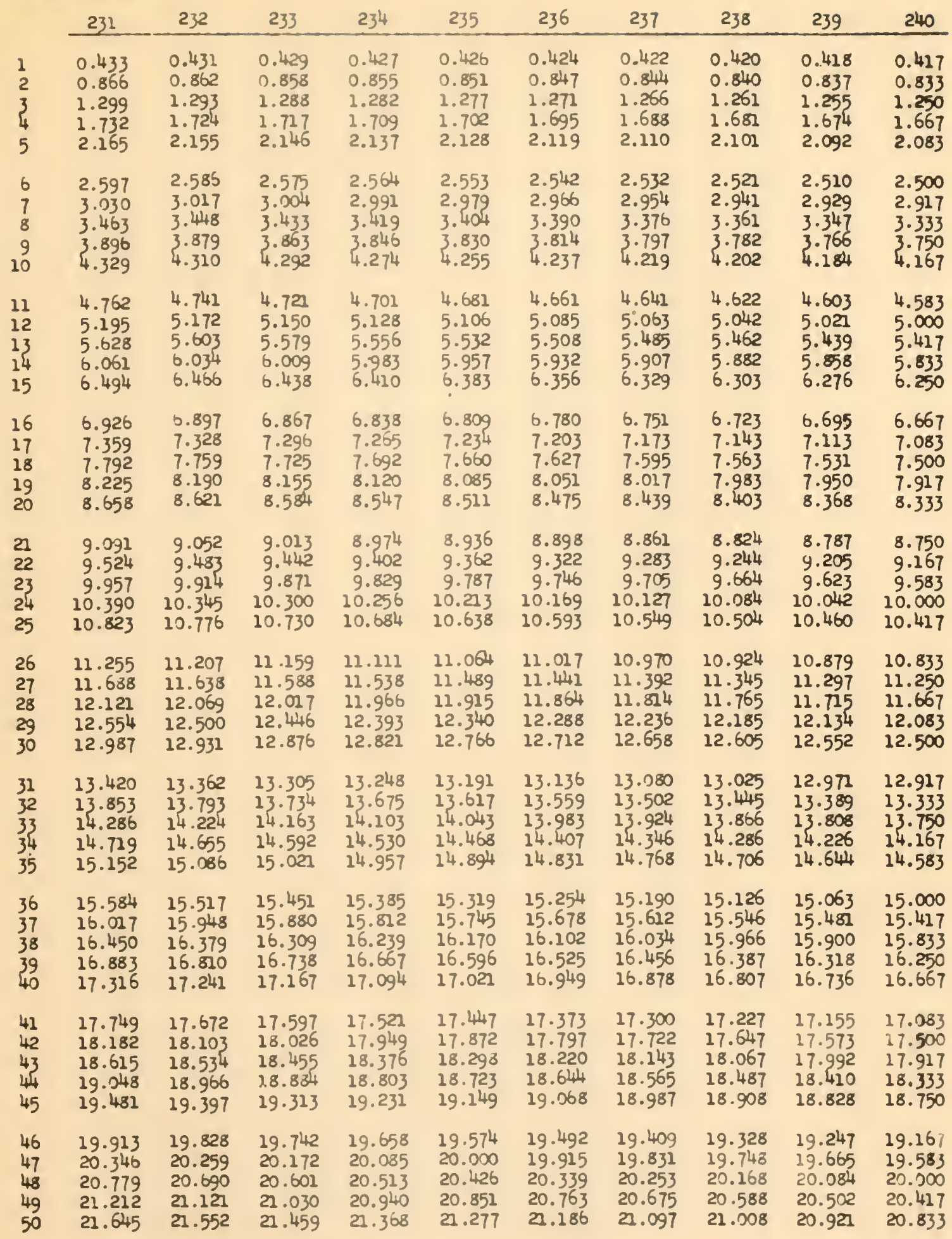



233

238

239

240

\section{1} 22.078 22.078
22.511 22.944 23.377 23.810

21.983

\subsection{8}

22.318

22.845

23.276

22.747

23.176

23.707

23.605

56

57

24.242

24.675

24.138

24.034

24.569

24.464

25.108

25.541

25.000

24.893

25.431

25.322

25.862

25.751

26.407

26.293

26.180

26.840

26. 724

27.273

27.706

27.155

27.586

26.609

27.039

27.468

27.897

28.139

66

67

28.571

29.004

29.437

29.870

28.017

28.448

28.879

29.310

29.741

23.326

28.755

29.185

29.614

30.303

30.172

30.736

30.603

31.169

31.602

32.035

31.034

31.466

31.897

32.328

30.043

30.472

30.901

31.330

31.700

32.189

32.900

32. 759

33.333

33.199

32.618

33.047

33.621

79

80

81

82

83

84

85

86

87

88

89

90

91

92

93

94

34.199
$34.63 ?$

34.052

34.483

33.470

33.906

34.335

35.065

34.914

35.498

35.931

36.364

35.345

34.754

35.193

35.622

35.770

36.207

36.052

36.797

36.638

36.481

37.229

37.069

37.662

38.095

38.528

38.961

37.50 n

37.931

38.362

38.793

36.910

37.339

37.708

38.197

38.627

39.394

39.827

40.260

40.693

41.126

39.224

39.655

40.086

40.51?

40.948

39.056

39.485

39.914

47.343

40.773

41.558

41.379

41.202

41.810

41.631

42.424

98

99

42.357

43.290

42.241

42.000

$42.672 \quad 42.489$

43.103

42.918
21.795

22.222

22.650

23.077

23.504

$23.932 \quad 23.830$

24.359

24.786

25.214

25.641

26.068

26.496

26.923

27.350

27.778

28.205

28.632

29.060

29.487

29.915

30.342

30.769

31.197

31.624

32.051

24.255

24.681

25.106

25.532

25.957

26.383

26.809

27.234

27.660

28.085

28.511

28.936

29.362

29.787

30.213

30.638

31.064

31.489

31.915

32.479

32.906

33.333

33.701

34.188

32.340

32.766

33.191

33.617

34.043

34.615

35.043

35.470

35.897

36.325

34.468

34.894

35.319

35.745

36.170

36.752

37.179

36.590

37.607

38.034

38.462

37.021

37.447

37.872

38.298

38.389

39.316

39.744

40.171

40.598

38.723

39.149

39.574

40.000

40.425

41.026

41.453

40.851

41.880

42.308

42.735
41.277

41.702

42.128

42.553

\section{$21.610 \quad 21.519$}

22.034

22.458

22.881

21.941

22.363

$22.7 \%$

23.207

23.305

23.629

23.729

24.153

24.051

24.576

24.473

25.000

24.895

25.316 



\begin{tabular}{|c|c|c|c|c|c|c|c|c|c|c|}
\hline & 231 & 232 & 233 & 234 & 235 & 236 & 237 & 238 & 239 & 240 \\
\hline 101 & 43.723 & 43.534 & 43.348 & 43.162 & 42.979 & 42.797 & 42.616 & 42.437 & 42.259 & 42.083 \\
\hline 102 & 44.156 & 43.966 & 43.777 & 43.590 & 43.404 & 43.220 & 43.038 & 42.857 & 42.678 & 42.500 \\
\hline 103 & 44.589 & 44.397 & 44.206 & 44.017 & 43.830 & 43.644 & 43.460 & 43.277 & 43.096 & 42.917 \\
\hline 104 & 45.022 & 44.828 & 44.635 & 44.444 & 44.255 & $4 \sqrt{4} .068$ & 43.882 & 43.697 & 43.515 & 43.333 \\
\hline 105 & 45.455 & 45.259 & 45.064 & 44.872 & 44.681 & 44.492 & 44.304 & 44.118 & 43.933 & 43.750 \\
\hline 06 & 45.887 & 45.690 & 45.494 & 45.299 & 45.106 & 44.915 & 44.726 & 44.538 & 44.351 & 44.167 \\
\hline 07 & 46.320 & 46.121 & 45.923 & 45.726 & 45.532 & 45.339 & 45.148 & 44.958 & 44.770 & 44.583 \\
\hline 08 & 46.753 & 46.552 & 46.352 & 46.154 & 45.957 & 45.763 & 45.570 & 45.378 & 45.188 & 45.000 \\
\hline 109 & 47.186 & 46.983 & 46.781 & 46.581 & 46.383 & 46.186 & 45.992 & 45.798 & 45.607 & $45: 417$ \\
\hline 110 & 47.619 & 47.414 & 47.210 & 47.009 & 46.809 & 46.610 & 46.414 & 46.218 & 46.025 & 45.833 \\
\hline 11 & 48.052 & 47.845 & 47.639 & 47.436 & 47.234 & 47.034 & 46.835 & 46.639 & 46.444 & 46.250 \\
\hline 12 & 48.485 & 48.276 & 48.069 & 47.863 & 47.660 & 47.458 & 47.257 & 47.059 & 46.862 & 46.667 \\
\hline 13 & 48.918 & 48.707 & 48.498 & 48.291 & 48.085 & 47.881 & 47.679 & 47.479 & 47.280 & 47.083 \\
\hline 14 & 49.351 & 49.138 & 48.927 & 48.718 & 48.511 & 48.305 & 48.101 & 47.899 & 47.699 & 47.500 \\
\hline 115 & 49.784 & 49.569 & 49.356 & 49.145 & 48.936 & 48.729 & 48.523 & 48.319 & 48.117 & 47.917 \\
\hline 16 & 50.216 & 50.000 & 49.785 & 49.573 & $49 \cdot 362$ & 49.153 & 48.945 & 48.739 & 48.536 & 48.333 \\
\hline 17 & 50.649 & 50.431 & 50.215 & 50.000 & .787 & 49.576 & 49.367 & 49.160 & 48.954 & 48.750 \\
\hline 18 & 51.082 & 50.862 & 50.644 & 50.427 & 50.213 & 50.000 & 49.789 & 49.580 & 49.372 & 49.167 \\
\hline 19 & 51.515 & 51.293 & 51.073 & 50.855 & 50.638 & 50.424 & 50.211 & 50.000 & 49.791 & 49.583 \\
\hline 20 & 51.948 & 51.724 & 51.502 & 51.282 & 51.064 & 50.847 & 50.633 & 50.420 & 50.209 & 50.000 \\
\hline 21 & 52.381 & 52.155 & 51.931 & 51.709 & 51.489 & 51.27 & 51.055 & 50.840 & 50.628 & 50.417 \\
\hline 122 & 52.814 & 52.586 & 52.361 & 52.137 & 51.915 & 51.695 & 51.477 & 51.261 & 51.046 & 50.833 \\
\hline 123 & 53.247 & 53.017 & 52.790 & 52.564 & 52.340 & 52.119 & 51.899 & 51.681 & 51.464 & 51.250 \\
\hline 24 & 53.680 & 53.448 & 53.219 & 52.991 & .766 & 52.542 & 52.321 & 52.101 & 51.883 & 51.667 \\
\hline 125 & 54.113 & 53.879 & 53.648 & 53.419 & 53.191 & 32.966 & 52.743 & 52.521 & 52.301 & 52.083 \\
\hline 26 & 54.545 & 54.310 & & 53.846 & 53.617 & 53.390 & 53.165 & 52.941 & 52.720 & 52.500 \\
\hline 27 & 54.978 & 54.741 & 54.506 & 54.274 & .043 & 53.814 & 53.586 & 53.361 & 53.13 & 52.917 \\
\hline 128 & 55.411 & 55.172 & 54.936 & 54.701 & .468 & 54.237 & 54.008 & 53.782 & 53.556 & 53.333 \\
\hline 129 & 55.844 & 55.603 & 55.365 & 55.128 & 54.894 & 54.661 & 54.430 & 54.202 & 53.975 & 53.750 \\
\hline 130 & 56.277 & 56.034 & 55.794 & 55.556 & 55.319 & 55.085 & 54.852 & 54.622 & 54.393 & 54.167 \\
\hline 31 & 56.710 & 56.466 & 56.223 & 55.983 & 55.745 & $55 \cdot 508$ & 55.274 & 55.042 & 54.812 & 54.583 \\
\hline 32 & 57.143 & 56.897 & 56.652 & 56.410 & 56.170 & 55.932 & 55.696 & 55.462 & 55.2 & .000 \\
\hline 33 & 57.576 & 57.328 & 57.082 & 56.838 & 56.596 & 56.356 & .118 & 55.882 & 55 & 55.417 \\
\hline 34 & 58.009 & 57.759 & 57.511 & 57.265 & 57.021 & 56.780 & 56.540 & 56.303 & 56.067 & 55.833 \\
\hline 135 & 58.442 & 58.190 & 57.940 & 57.692 & & 57.203 & & 56.723 & 56.485 & 56.250 \\
\hline 136 & 58.874 & 58.621 & 58.369 & 58.120 & 57.872 & 57.627 & 57.384 & 57.143 & 56.904 & 56.667 \\
\hline 37 & 59.307 & 59.052 & 58.798 & 58.547 & 58.298 & 58.051 & .806 & 57.563 & $57 \cdot 322$ & 57.083 \\
\hline 138 & & 59.483 & 59.227 & 58.974 & .723 & 58.475 & .228 & 57.983 & 57.74 & 57.500 \\
\hline 139 & 60.173 & 59.914 & 59.657 & 59.402 & 59.149 & 58.898 & 58.650 & 58.403 & 58.15 & 57.917 \\
\hline & 6 & $60 \cdot 345$ & 60.086 & 59.829 & 59.574 & $59 \cdot 322$ & 59.072 & 58 & 58.577 & \\
\hline 141 & 61.039 & 60.776 & 60.515 & 60.256 & 60.000 & 59.746 & 59.494 & 59.244 & 58.99 & 58.750 \\
\hline 142 & 61.472 & 61.207 & 60.944 & 60.684 & 60.426 & 60.169 & $59 \cdot 916$ & 59.664 & & 59.167 \\
\hline 143 & 61.905 & 61.638 & 61.373 & 61.111 & 60.851 & 63.593 & 60.338 & 60.084 & 59.833 & 59.583 \\
\hline 144 & 62.338 & 62.069 & 61.803 & 61.538 & 61.277 & 61.017 & 60.759 & 60.504 & & 60.000 \\
\hline 14 & 62.771 & 62.500 & 62.232 & 61.966 & 61.702 & 61.442 & 61.181 & 60.924 & 60.669 & 60.417 \\
\hline 146 & 63.203 & 62.931 & 62.661 & 62.393 & 62.128 & 52.864 & 61.603 & 61.345 & 61.088 & 60.833 \\
\hline 147 & 63.636 & 63.3621 & 63.090 & 62.821 & 62.553 & 62.288 & 62.025 & 61.765 & 61.506 & 61.250 \\
\hline 148 & 64.069 & 63.793 & 63.519 & 63.248 & 62 & 62.712 & 62.447 & 62.185 & 61.925 & 61.667 \\
\hline & 64.502 & 64.224 & 63.948 & 63.675 & 63.404 & 63.136 & 62.869 & 62.605 & 62.343 & 62.083 \\
\hline 5 & 64.935 & 64.655 & 64.378 & 64.103 & 63.830 & 63.559 & 63.291 & 63.025 & 62.762 & 62.500 \\
\hline
\end{tabular}





$\begin{array}{lllllllll}231 & 232 & 233 & 234 & 235 & 236 & 237 & 238 & 239\end{array}$

\begin{tabular}{|c|c|c|c|c|c|c|c|c|c|c|}
\hline $\begin{array}{l}151 \\
152 \\
153 \\
154 \\
155\end{array}$ & $\begin{array}{l}65.368 \\
65.801 \\
66.234 \\
66.667 \\
67.100\end{array}$ & $\begin{array}{l}65.086 \\
65.517 \\
65.948 \\
66.379 \\
66.810\end{array}$ & $\begin{array}{l}64.807 \\
65.236 \\
65.665 \\
66.094 \\
66.524\end{array}$ & $\begin{array}{l}64.530 \\
64.957 \\
65.385 \\
65.812 \\
66.239\end{array}$ & $\begin{array}{l}64.255 \\
64.681 \\
65.106 \\
65.532 \\
65.957\end{array}$ & $\begin{array}{l}63.983 \\
64.407 \\
64.831 \\
65.254 \\
65.678\end{array}$ & $\begin{array}{l}63.723 \\
64.135 \\
64.557 \\
64.979 \\
65.401\end{array}$ & $\begin{array}{l}63.445 \\
63.866 \\
64.286 \\
64.706 \\
65.126\end{array}$ & $\begin{array}{l}63.180 \\
63.598 \\
64.017 \\
64.435 \\
64.854\end{array}$ & $\begin{array}{l}62.917 \\
63.333 \\
63.750 \\
64.167 \\
64.583\end{array}$ \\
\hline $\begin{array}{l}130 \\
159 \\
160\end{array}$ & $\begin{array}{l}67.532 \\
67.965 \\
68.398 \\
68.831 \\
69.264\end{array}$ & $\begin{array}{l}67.241 \\
67.672 \\
68.103 \\
68.534 \\
68.966\end{array}$ & $\begin{array}{l}66.953 \\
67.382 \\
67.811 \\
68.240 \\
68.670\end{array}$ & $\begin{array}{l}66.667 \\
67.094 \\
67.521 \\
67.949 \\
68.376\end{array}$ & $\begin{array}{l}66.383 \\
66.809 \\
67.234 \\
67.660 \\
68.085\end{array}$ & $\begin{array}{l}66.102 \\
66.525 \\
66.949 \\
67.373 \\
67.797\end{array}$ & $\begin{array}{l}65.823 \\
66.245 \\
66.667 \\
67.089 \\
67.511\end{array}$ & $\begin{array}{l}65.546 \\
65.966 \\
66.387 \\
66.807 \\
67.227\end{array}$ & $\begin{array}{l}65.272 \\
65.690 \\
66.109 \\
66.527 \\
66.946\end{array}$ & $\begin{array}{l}65.000 \\
65.417 \\
65.833 \\
66.250 \\
66.667\end{array}$ \\
\hline $\begin{array}{l}163 \\
164 \\
165\end{array}$ & $\begin{array}{l}69.697 \\
70.130 \\
70.563 \\
70.996 \\
71.429\end{array}$ & $\begin{array}{l}69.397 \\
69.828 \\
70.259 \\
70.690 \\
71.121\end{array}$ & $\begin{array}{l}69.099 \\
69.528 \\
69.957 \\
70.386 \\
70.815\end{array}$ & $\begin{array}{l}68.803 \\
69.231 \\
69.658 \\
70.085 \\
70.513\end{array}$ & $\begin{array}{l}68.511 \\
68.936 \\
69.362 \\
69.787 \\
70.213\end{array}$ & $\begin{array}{l}68.220 \\
68.644 \\
69.068 \\
69.492 \\
69.915\end{array}$ & $\begin{array}{l}67.932 \\
68.354 \\
68.776 \\
69.198 \\
69.620\end{array}$ & $\begin{array}{l}67.647 \\
68.067 \\
68.487 \\
68.908 \\
69.328\end{array}$ & $\begin{array}{l}67.361 \\
67.782 \\
68.201 \\
68.619 \\
69.038\end{array}$ & $\begin{array}{l}67.083 \\
67.500 \\
67.917 \\
68.333 \\
68.750\end{array}$ \\
\hline $\begin{array}{l}166 \\
167 \\
168 \\
169 \\
170\end{array}$ & $\begin{array}{l}71.861 \\
72.294 \\
72.727 \\
73.160 \\
73.593\end{array}$ & $\begin{array}{l}71.552 \\
71.983 \\
72.414 \\
72.845 \\
73.276\end{array}$ & $\begin{array}{l}71.245 \\
71.674 \\
72.103 \\
72.532 \\
72.961\end{array}$ & $\begin{array}{l}70.940 \\
71.368 \\
71.795 \\
72.222 \\
72.650\end{array}$ & $\begin{array}{l}70.638 \\
71.0641 \\
71.489 \\
71.915 \\
72.340\end{array}$ & $\begin{array}{l}70.339 \\
70.763 \\
71.186 \\
71.610 \\
72.034\end{array}$ & $\begin{array}{l}70.042 \\
70.464 \\
70.886 \\
71.308 \\
71.730\end{array}$ & $\begin{array}{l}69.748 \\
70.168 \\
70.588 \\
71.008 \\
71.429\end{array}$ & $\begin{array}{l}69.456 \\
69.874 \\
70.293 \\
70.711 \\
71.130\end{array}$ & $\begin{array}{l}69.167 \\
69.583 \\
70.000 \\
70.417 \\
70.833\end{array}$ \\
\hline $\begin{array}{l}17 \\
172 \\
173 \\
174 \\
175\end{array}$ & $\begin{array}{l}74.026 \\
74.459 \\
74.892 \\
75.325 \\
75.758\end{array}$ & $\begin{array}{l}73.707 \\
74.138 \\
74.569 \\
75.000 \\
75.431\end{array}$ & $\begin{array}{l}73.391 \\
73.820 \\
74.249 \\
74.678 \\
75.107\end{array}$ & $\begin{array}{l}73.077 \\
73.504 \\
73.932 \\
74.359 \\
74.786\end{array}$ & $\begin{array}{l}72.766 \\
73.191 \\
73.617 \\
74.043 \\
74.468\end{array}$ & $\begin{array}{l}72.458 \\
72.881 \\
73.305 \\
73.729 \\
74.153\end{array}$ & $\begin{array}{l}72.152 \\
72.574 \\
72.996 \\
73.418 \\
73.840\end{array}$ & $\begin{array}{l}71.849 \\
72.269 \\
72.689 \\
73.109 \\
73.529\end{array}$ & $\begin{array}{l}71.548 \\
71.967 \\
72.385 \\
72.803 \\
73.222\end{array}$ & $\begin{array}{l}7.250 \\
71.667 \\
72.083 \\
72.500 \\
72.917\end{array}$ \\
\hline $\begin{array}{l}176 \\
177 \\
178 \\
179 \\
180\end{array}$ & $\begin{array}{l}76.190 \\
76.623 \\
77.056 \\
77.489 \\
77.922\end{array}$ & $\begin{array}{l}75.862 \\
76.293 \\
76.724 \\
77.155 \\
77.586\end{array}$ & $\begin{array}{l}75.536 \\
75.966 \\
76.395 \\
76.824 \\
77.253\end{array}$ & $\begin{array}{l}75.214 \\
75.641 \\
76.068 \\
76.496 \\
76.923\end{array}$ & $\begin{array}{l}74.894 \\
75.319 \\
75.745 \\
76.170 \\
76.596\end{array}$ & $\begin{array}{l}74.576 \\
75.000 \\
75.424 \\
75.847 \\
76.271\end{array}$ & $\begin{array}{l}74.262 \\
74.684 \\
75.105 \\
75.527 \\
75.949\end{array}$ & $\begin{array}{l}73.950 \\
74.370 \\
74.790 \\
75.210 \\
75.630\end{array}$ & $\begin{array}{l}73.640 \\
74.059 \\
74.477 \\
74.895 \\
75.314\end{array}$ & $\begin{array}{l}73.333 \\
73.750 \\
74.167 \\
74.583 \\
75.000\end{array}$ \\
\hline $\begin{array}{l}181 \\
182 \\
183 \\
184 \\
185\end{array}$ & $\begin{array}{l}78.355 \\
78.788 \\
79.221 \\
79.654 \\
80.087\end{array}$ & $\begin{array}{l}78.017 \\
78.448 \\
78.879 \\
79.310 \\
79.741\end{array}$ & $\begin{array}{l}77.682 \\
78.112 \\
78.541 \\
78.970 \\
79.399\end{array}$ & $\begin{array}{l}77.350 \\
77.778 \\
78.205 \\
78.632 \\
79.060\end{array}$ & $\begin{array}{l}77.021 \\
77.447 \\
77.872 \\
78.298 \\
78.723\end{array}$ & $\begin{array}{l}76.695 \\
77.219 \\
77.542 \\
77.966 \\
78.390\end{array}$ & $\begin{array}{l}76.371 \\
76.793 \\
77.215 \\
77.637 \\
78.059\end{array}$ & $\begin{array}{l}76.050 \\
76.471 \\
76.891 \\
77.311 \\
77.731\end{array}$ & $\begin{array}{l}75.732 \\
76.151 \\
76.569 \\
76.987 \\
77.406\end{array}$ & $\begin{array}{l}75.417 \\
75.833 \\
76.250 \\
76.667 \\
77.083\end{array}$ \\
\hline $\begin{array}{l}186 \\
187 \\
188 \\
189 \\
190\end{array}$ & $\begin{array}{l}80.519 \\
80.952 \\
81.385 \\
81.818 \\
82.251\end{array}$ & $\begin{array}{l}80.172 \\
80.603 \\
81.034 \\
81.466 \\
81.897\end{array}$ & $\begin{array}{l}79.828 \\
80.258 \\
80.687 \\
81.116 \\
81.545\end{array}$ & $\begin{array}{l}79.487 \\
79.915 \\
80.342 \\
80.769 \\
8.197\end{array}$ & $\begin{array}{l}79.149 \\
79.574 \\
80.000 \\
80.426 \\
80.851\end{array}$ & $\begin{array}{l}78.814 \\
79.237 \\
79.661 \\
80.085 \\
80.508\end{array}$ & $\begin{array}{l}78.481 \\
78.903 \\
79.325 \\
79.747 \\
80.169\end{array}$ & $\begin{array}{l}78.151 \\
78.571 \\
78.992 \\
79.412 \\
79.832\end{array}$ & $\begin{array}{l}77.824 \\
78.243 \\
78.661 \\
79.079 \\
79.498\end{array}$ & $\begin{array}{l}77.500 \\
77.917 \\
78.333 \\
78.750 \\
79.167\end{array}$ \\
\hline $\begin{array}{l}191 \\
192 \\
193 \\
194 \\
195\end{array}$ & $\begin{array}{l}82.684 \\
83.117 \\
83.550 \\
83.983 \\
84.416\end{array}$ & $\begin{array}{l}82.328 \\
82.759 \\
83.190 \\
83.621 \\
84.052\end{array}$ & $\begin{array}{l}81.974 \\
82.403 \\
82.833 \\
83.262 \\
83.691\end{array}$ & $\begin{array}{l}81.624 \\
82.051 \\
82.479 \\
82.906 \\
83.333\end{array}$ & $\begin{array}{l}81.277 \\
81.702 \\
82.128 \\
82.553 \\
82.979\end{array}$ & $\begin{array}{l}80.932 \\
81.356 \\
81.780 \\
82.203 \\
82.627\end{array}$ & $\begin{array}{l}80.591 \\
81.013 \\
81.435 \\
81.857 \\
82.278\end{array}$ & $\begin{array}{l}80.252 \\
80.672 \\
81.092 \\
81.513 \\
81.933\end{array}$ & $\begin{array}{l}79.916 \\
80.335 \\
80.753 \\
81.172 \\
81.590\end{array}$ & $\begin{array}{l}79.583 \\
80.000 \\
80.417 \\
80.833 \\
81.250\end{array}$ \\
\hline $\begin{array}{l}196 \\
197 \\
198 \\
199 \\
200\end{array}$ & $\begin{array}{l}84.848 \\
85.281 \\
85.714 \\
86.147 \\
86.580\end{array}$ & $\begin{array}{l}84.483 \\
84.914 \\
8.345 \\
85.776 \\
86.207\end{array}$ & $\begin{array}{l}84.120 \\
84.549 \\
84.979 \\
85.408 \\
85.837\end{array}$ & $\begin{array}{l}83.761 \\
84.188 \\
84.615 \\
85.043 \\
85.470\end{array}$ & $\begin{array}{l}83.404 \\
83.830 \\
84.255 \\
84.681 \\
85.106\end{array}$ & $\begin{array}{l}83.051 \\
83.475 \\
83.898 \\
84.322 \\
84.746\end{array}$ & $\begin{array}{l}82.700 \\
83.122 \\
83.544 \\
83.966 \\
84.388\end{array}$ & $\begin{array}{l}82.353 \\
82.773 \\
83.193 \\
83.613 \\
84.034\end{array}$ & $\begin{array}{l}82.008 \\
82.427 \\
82.845 \\
83.264 \\
83.682\end{array}$ & $\begin{array}{l}81.667 \\
82.083 \\
82.500 \\
82.917 \\
83.333\end{array}$ \\
\hline
\end{tabular}



PERCEITAGR TABLES

\begin{tabular}{llllllllll}
231 & 232 & 233 & 231 & 235 & 236 & 237 & 238 & 239 & 240 \\
\hline
\end{tabular}

\begin{tabular}{|c|c|c|c|c|c|c|c|c|c|c|}
\hline 201 & 87.013 & 86.638 & 86.266 & 85.397 & 85.532 & 85.169 & 84.810 & 84.454 & 84.100 & 83.750 \\
\hline 202 & 87.446 & 87.069 & 86.695 & 86.325 & 85.957 & 85.593 & 85.232 & 84.874 & 84.519 & 84.167 \\
\hline 203 & 87.879 & 87.500 & 87.124 & 86.752 & 86.383 & 86.017 & 85.654 & 85.294 & 84.937 & 84.583 \\
\hline 204 & 88.312 & 87.931 & 87.554 & 87.179 & 86.809 & 86.441 & 86.076 & 85.714 & 85.356 & 85.000 \\
\hline 205 & 88.745 & 88.362 & 87.983 & 87.607 & 87.234 & 86.864 & 86.498 & 86.134 & 85.774 & 85.417 \\
\hline 206 & 89.177 & 88.793 & 88.412 & 88.034 & 87.660 & 87.288 & 86.920 & 86.555 & 86.192 & 85.833 \\
\hline 207 & 89.610 & 89.224 & 88.841 & 462 & 38.085 & 87.712 & 87.342 & 86. & & 86.250 \\
\hline 208 & 90.043 & 89.655 & 89.270 & 88.889 & 88.511 & 88.136 & 87.764 & 87.395 & 87.029 & 86.667 \\
\hline 209 & 90.476 & 90.086 & 89.700 & 89.316 & 88.936 & 88.559 & 88.186 & 87.8 & 87.448 & 87.083 \\
\hline 210 & 90.909 & 90.517 & 90.129 & 89.744 & 89.362 & 88.983 & 88.608 & 88.235 & 87.866 & 87.500 \\
\hline 211 & 91.342 & 90.948 & 90.558 & 90.171 & 89.787 & 89.407 & 89.030 & 88.655 & 88.285 & 87.917 \\
\hline 212 & 91.775 & 91.379 & 90.987 & & 90.213 & 331 & .451 & 89.076 & .703 & 88.333 \\
\hline 213 & 92.208 & 91.810 & 91.416 & 91.026 & 90.638 & 90.254 & 89.873 & 89.496 & 89.121 & 88.750 \\
\hline 214 & 92.641 & 92.241 & 91.845 & 91. & 91.064 & 90.678 & 90.295 & 89.916 & & 89.167 \\
\hline 215 & 93.074 & 92.672 & 92.275 & 91.880 & 91.489 & 91.102 & 90.717 & 90.336 & 89.958 & 89.583 \\
\hline 215 & 93.506 & 93.103 & 92.704 & 92.308 & 91.915 & 91.525 & 91.139 & 90.756 & 90.377 & 90.000 \\
\hline י7 & 93.939 & 93.534 & 93.133 & 92.735 & 92.340 & 91.949 & 91.561 & 91.176 & 90.795 & 90.417 \\
\hline 8 & 94.372 & 93.966 & 93.562 & 93.162 & 92.766 & 92.373 & 91.983 & 91. & 91.2 & 90.833 \\
\hline 219 & 94.805 & 94.397 & 93.991 & 93.590 & 93.191 & 92.797 & 92.405 & 92.017 & 91.632 & 91.250 \\
\hline 220 & 95.238 & 94.828 & 94.421 & 94.017 & 93.617 & 93.220 & 92.827 & 92.437 & 92.050 & 91.667 \\
\hline 227 & 95.677 & 95.259 & 94.850 & 94.444 & 94.043 & 93.644 & 93.249 & 92.857 & 92.469 & 92.083 \\
\hline 222 & 96.104 & & 95.2 & 94. & & & & 93. & & 500 \\
\hline 223 & 96.537 & 96.121 & 95.708 & 95.299 & 94.894 & 94.492 & 94.093 & 93.697 & 93.305 & 92.917 \\
\hline 224 & 96.970 & 96.552 & 96.137 & 95.726 & 95.319 & 94.915 & 94.515 & 94.118 & 93. & 93.333 \\
\hline 225 & 97.403 & 96.983 & 96.567 & 96.154 & 95.745 & 95.339 & 94.937 & 94.538 & 94.142 & 93.750 \\
\hline 226 & 97.835 & 97.414 & 96.996 & 96.581 & 96.170 & 95.763 & 95.359 & 94.958 & 94.561 & 94.167 \\
\hline 227 & 98.268 & 97.845 & 97.4 & 97.009 & 96.596 & 96.186 & 95.781 & 95.378 & 94.979 & 94.583 \\
\hline 228 & 98.701 & 98.276 & 97.854 & 97.436 & 97.021 & 96.610 & 96.203 & 95.798 & 95. & 95.000 \\
\hline 229 & 99.134 & 98.707 & 98.283 & 97.863 & 97. & 97.034 & 96.624 & 96.2 & 816 & 417 \\
\hline 230 & 99.567 & 99.138 & 98.712 & 98.291 & 97.872 & & 97.046 & 96.639 & 96.234 & 95.833 \\
\hline 231 & 100.000 & 99.569 & 99.142 & 98.718 & 98.298 & 97.881 & 97.468 & 97.059 & 96.653 & 96.25 \\
\hline 23 & -- & 100.000 & & & & & & & & 667 \\
\hline 233 & $\cdots$ & --- & 100.000 & 99.573 & 99.149 & 98.729 & 98.312 & 97.899 & 97.490 & 97.083 \\
\hline 234 & --- & - & $\cdots$ & 100.000 & 99.574 & 99.1 & 98.734 & 98.319 & 97.908 & 97.500 \\
\hline 235 & $\cdots$ & $-\infty$ & $-\infty$ & $\cdots$ & 100.000 & 99.576 & 99.156 & 98.739 & 98.326 & 97.917 \\
\hline 236 & --- & $-\infty$ &.- & - & $-\infty$ & 100.000 & 99.578 & 99.160 & 98.745 & 98.333 \\
\hline 237 & $\cdots$ & $\ldots$ & --- & $\ldots$ &.-- & $\ldots$ & 100.000 & 99.580 & 99.163 & 98.750 \\
\hline 238 & -- & -- & $\ldots$ & - & $\cdots$ & $\cdots$ & -- & 100.000 & 99.582 & 99.167 \\
\hline 239 & $\cdots$ & $\cdots$ & -.. &.-- & -- & -.- & -- & $\cdots$ & 100.000 & 99.583 \\
\hline & -.- & $\ldots$ & -.- & -.. & -.. & $\ldots$ & -.. & -.. & --- & 100.000 \\
\hline
\end{tabular}





\begin{tabular}{|c|c|c|c|c|c|c|c|c|c|c|}
\hline & 241 & 242 & 243 & 244 & 245 & 246 & 247 & 248 & 249 & 250 \\
\hline 1 & 0.415 & 0.413 & 0.432 & 0.410 & 0.408 & 0.407 & 0.405 & 0.403 & 0.402 & 0.400 \\
\hline 2 & 0.830 & 0.826 & 0.823 & 0.820 & 0.816 & 0.813 & 0.810 & & 0.803 & 0.800 \\
\hline 3 & 1.245 & 1.240 & 1.235 & 1.230 & 1.224 & 1.220 & 1.225 & & 1.205 & 1.200 \\
\hline 4 & 1.66 & 1.653 & 1.646 & 1.639 & 1.633 & 1.626 & 1.619 & 1.613 & 1.606 & 1.600 \\
\hline 5 & 2.075 & 2.066 & 2.058 & 2.049 & 2.041 & 2.033 & 2.024 & 2.016 & 2.008 & 2.000 \\
\hline 6 & 2.490 & 2.479 & 2.469 & 2.459 & 2.449 & 2.439 & 2.429 & 2.419 & 2.410 & 2.400 \\
\hline 7 & 2.905 & 2.893 & 2.881 & 2.869 & 2.857 & 2.846 & 2.8 & & & 2.800 \\
\hline 8 & 3.320 & 3.306 & 3.292 & 3.279 & 3.265 & 3.252 & 3.239 & & & 3.200 \\
\hline 9 & 3.734 & 3.719 & 3.704 & 3.689 & 3.673 & 3.659 & 3.644 & 3.629 & & 3.600 \\
\hline 10 & 4.149 & 4.132 & 4.115 & 4.098 & 4.082 & 4.065 & 4.0109 & 4.032 & 4.916 & 4.000 \\
\hline 11 & 4.564 & 4.545 & 4.527 & 4.508 & 4.490 & 4.472 & 4.453 & 4.435 & 4.418 & 4.400 \\
\hline & 4.979 & .959 & 4.938 & 4.918 & 4.898 & 4.8 & 4.858 & & & 4.800 \\
\hline S & 5.394 & 5.372 & 5.350 & 5.328 & 5.306 & 5.285 & 5.263 & 5.242 & & 5.200 \\
\hline 4 & $\begin{array}{l}5.809 \\
6.224\end{array}$ & $\begin{array}{l}5.785 \\
6.198\end{array}$ & 5.761 & $\begin{array}{l}5.738 \\
6.148\end{array}$ & $\begin{array}{l}5.714 \\
6.122\end{array}$ & 5.691 & 5.668 & $\begin{array}{l}5.6 \\
6.0\end{array}$ & $\begin{array}{l}5.6 \\
6.0\end{array}$ & 5.600 \\
\hline & & & & 0.1 & & 0.0 & 0.0 & & & 6.000 \\
\hline 16 & 6.639 & 6.612 & 6.584 & 6.557 & 6.531 & 6.504 & 6.478 & 6.452 & 6.426 & 6.400 \\
\hline & 7.054 & 7.025 & 6.996 & 6.9 & 6.9 & 6.9 & 6.8 & & 6.827 & 6.800 \\
\hline 8 & 7.469 & 7.438 & 7.407 & 7.377 & 7.3 & 7.3 & 7.2 & & & 7.200 \\
\hline 19 & 7.884 & 7.851 & 7.819 & 7.787 & 7.7 & 7.724 & 7.692 & 7.6 & 7.6 & 7.600 \\
\hline 20 & 8.299 & 8.264 & 8.230 & 8.197 & 8.163 & 8.130 & 8.097 & 8.065 & 8.032 & 8.000 \\
\hline 21 & 8.714 & 8.678 & 8.642 & 8.607 & 8.571 & 8.537 & 8.50 & 8.468 & 8.4314 & $8.4 \mathrm{~m}$ \\
\hline 22 & 9.129 & 9.091 & 9.053 & 9.016 & 8.98 & 8.9 & 8.9 & 8.8 & 8.835 & 8.800 \\
\hline & 9.544 & 9.504 & 9.465 & 9.426 & 9.388 & 9.3 & 9.3 & 9. & 9.2 & 9.200 \\
\hline 24 & 9.959 & 9.917 & 9.877 & 9.836 & 9.796 & 9.756 & 9.7 & 9.1 & & 9.600 \\
\hline 25 & 10.373 & 10.331 & 10.288 & 10.246 & 10.204 & 10.163 & 10.121 & 10.081 & 10.040 & 10.000 \\
\hline 26 & 0.788 & 0.744 & 10.700 & 10.656 & 10.612 & 10.569 & 10.526 & & 10.442 & 10.400 \\
\hline c) & .203 & .157 & 11 & .066 & 11.0 & 6 & 31 & 37 & 3 & \\
\hline 28 & 11.618 & .570 & 11.5 & 15 & 9 & 1 & 1.3 & 11. & 5. & 1.200 \\
\hline 29 & 12.033 & .983 & 11.934 & 11.885 & 11.8 & 11.789 & 11.741 & 1.694 & & 11.600 \\
\hline 30 & 12.448 & 12.397 & 12.346 & 12.295 & 12.245 & 12.195 & 12.146 & 12.097 & 12.048 & 12.000 \\
\hline 31 & 12.867 & 12.810 & & & 126 & & & & & \\
\hline & 13.278 & 13.223 & 13 & 5 & 1 & 13. & 55 & 12.9 & 12.8 & .800 \\
\hline 33 & 13.693 & 13.636 & 13.58 & 5 & 1. & 13.4 & 1 & & & 3.200 \\
\hline 34 & 14.108 & 14.050 & 13.992 & 17 & & & & & & 3.600 \\
\hline 35 & 14.523 & 14.463 & 14.403 & 14.344 & 14.286 & 14.228 & 14.170 & 14.113 & 14.056 & 14.000 \\
\hline 36 & .938 & 14.876 & & 14.754 & & & & & 14.45 & \\
\hline & 353 & & & & & & & & & \\
\hline & 768 & .702 & 15.63 & 16 & & & 1 & & & 200 \\
\hline & .183 & & 16 & & & & & & & \\
\hline & 16.598 & .529 & .461 & 393 & & 16.260 & & 16.129 & 16.064 & 16.000 \\
\hline 41 & 17.012 & 6.942 & 16.8 & 16.8 & 16.7 & 16.6 & 16.5 & & & 16.400 \\
\hline & 17.427 & 7.355 & 17.284 & 17.213 & 17.1 & & & & & 16.800 \\
\hline & & & 17.60 & 17.623 & & & & & & 17.200 \\
\hline & 18.257 & 18.1 & & 18.03 & & & & & & \\
\hline 45 & 18.672 & 18.595 & 18.519 & 18.443 & 18.367 & 18.293 & 18.219 & 18.145 & 18.072 & 18.000 \\
\hline 46 & & & & & & & & & & 18.400 \\
\hline & & & & & & & & & & \\
\hline 48 & 917 & & & 19 & & 19.5 & & & & 19.200 \\
\hline 49 & 20.332 & & & & & & & & & 19.600 \\
\hline & 20.747 & 20.661 & 20.576 & 20.492 & 20.408 & 20.325 & 20.243 & 20.161 & 20.080 & 20.000 \\
\hline
\end{tabular}





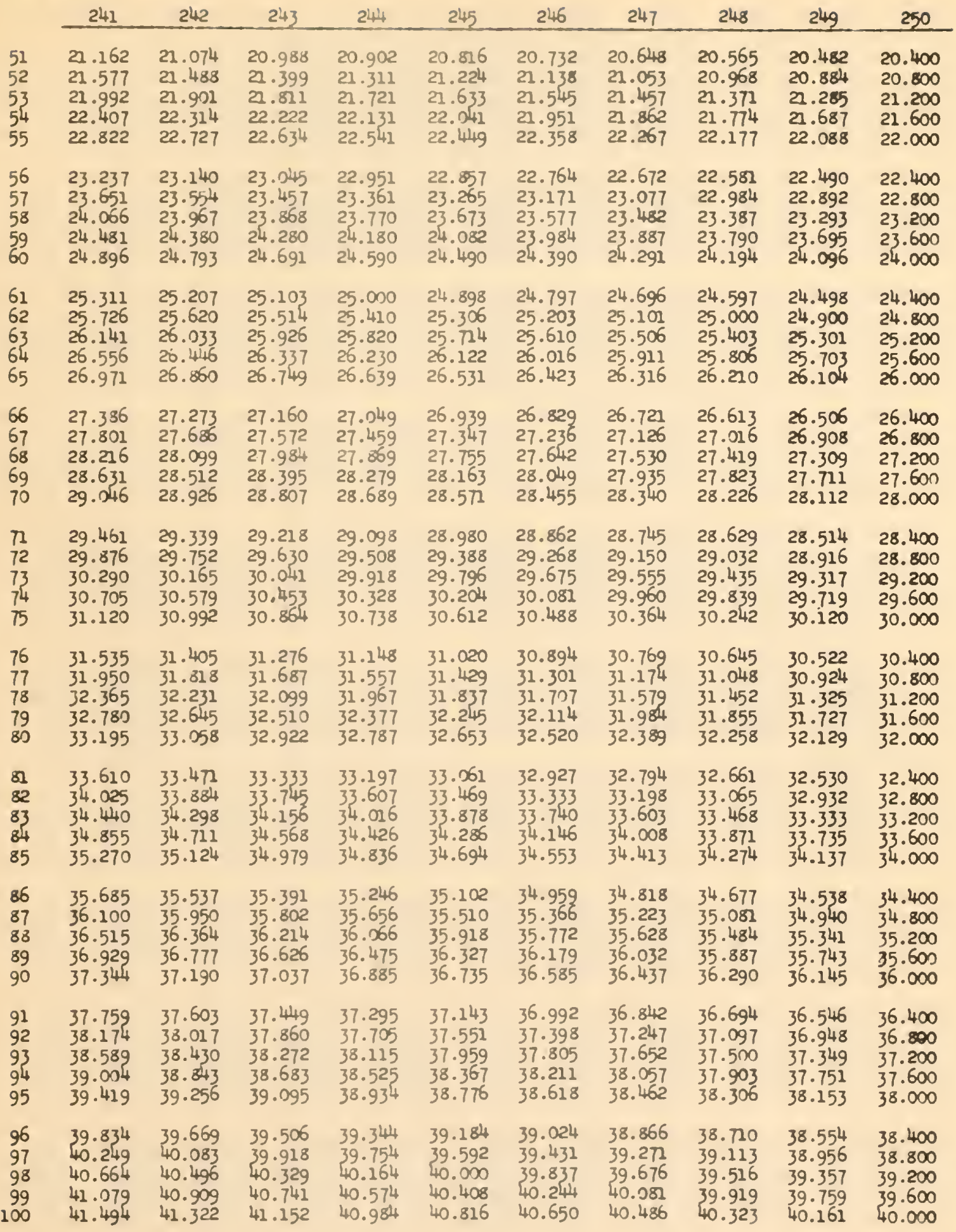





\begin{tabular}{|c|c|c|c|c|c|c|c|c|c|c|}
\hline & 241 & 242 & 243 & 244 & 245 & 246 & 247 & 248 & 249 & 250 \\
\hline 101 & 41.909 & 41.736 & 41.564 & 41.393 & 41.224 & 41.057 & 40.891 & 140.726 & 40.562 & 40.400 \\
\hline 102 & 42.324 & 42.149 & 41.975 & 42.803 & 41.633 & 41.463 & 41.296 & 41.129 & 40.964 & 40.800 \\
\hline 103 & 42.739 & 42.562 & 42.387 & 42.213 & 42.041 & 41.870 & 41.700 & 41.532 & 41.365 & 41.200 \\
\hline 104 & 43.154 & 42.975 & 42.798 & 42.623 & 42.449 & 42.276 & 42.105 & 41.935 & 41.767 & 41.600 \\
\hline 105 & 43.568 & 43.388 & 43.210 & 43.033 & 42.857 & 42.683 & 42.510 & 42.339 & 42.169 & 42.000 \\
\hline$\infty$ & 43.983 & 43.802 & 43.621 & 43.443 & 3.265 & 43.089 & 2.915 & 42.742 & 42.570 & 42.400 \\
\hline 07 & 4.398 & 44.215 & 44.033 & 43.852 & 43.673 & 43.496 & 43.320 & 43.145 & 42.972 & 42.800 \\
\hline 108 & 44.813 & 44.628 & 44.444 & 44.262 & 44.082 & 43.902 & 43.725 & 43.548 & 43.373 & 43.200 \\
\hline 09 & 45.228 & 45.041 & 44.856 & 44.672 & 44.490 & $4 \sqrt{4} 309$ & 44.130 & 43.952 & 43.775 & 43.600 \\
\hline 10 & 45.643 & 45.455 & 45.267 & 45.082 & 44.898 & 44.715 & 44.534 & 44.355 & .177 & 44.000 \\
\hline 11 & 46.058 & 45.868 & 45.679 & 45.492 & 45.306 & 45.122 & 44.939 & 44.758 & 44.578 & 44.400 \\
\hline 12 & 46.473 & 46.281 & 46.091 & 45.902 & 45.714 & 45.528 & $45 \cdot 344$ & 45.161 & 44.980 & 44.800 \\
\hline 3 & 46.838 & 46.694 & 46.502 & 46.311 & 46.122 & 45.935 & 45.749 & 45.565 & 45.382 & 45.200 \\
\hline 4 & 47.303 & 47.107 & 46.914 & 46.721 & 46.531 & 46.341 & 46.154 & 45.968 & 45.783 & 45.600 \\
\hline 15 & 47.718 & 47.521 & 47.325 & 47.131 & 46.939 & 46.748 & 46.559 & 46.37 & 46.185 & 46.000 \\
\hline 16 & 48.133 & 47.934 & 47.737 & 47.541 & $47 \cdot 347$ & 47.154 & 46.964 & 46.774 & 46.586 & 46.400 \\
\hline 7 & 48.548 & 48.347 & 48.148 & 47.951 & 47.755 & 47.561 & 47.368 & 47.177 & 46.988 & 46.800 \\
\hline 18 & 48.963 & 48.760 & 48.560 & 48.361 & 48.163 & 47.967 & 47.773 & 47.581 & 47.390 & 47.200 \\
\hline 19 & 49.378 & 49.174 & 48.971 & 48.770 & $48.5 \pi$ & 48.374 & .178 & 47.984 & 47.791 & 47.600 \\
\hline 20 & 49.793 & 49.587 & 49.383 & 49.180 & 48.980 & 48.780 & 48.583 & 48.387 & 48.193 & 48.000 \\
\hline 21 & 50.207 & 50.000 & 49.794 & 49.590 & 49.388 & 49.187 & 988 & 48.790 & 48.594 & 48.400 \\
\hline 22 & 50.622 & 50.413 & 50.206 & 50.000 & 49.796 & 49.593 & 49.393 & 49.194 & 48.996 & 48.800 \\
\hline 23 & 51.037 & 50.826 & 50.617 & 50.410 & 50.204 & 50.000 & 49.798 & 49.597 & 49.398 & 49.200 \\
\hline 24 & 51.452 & 51.240 & 51.029 & 50.820 & 50.612 & 50.407 & 50.202 & 50.0 & 49.799 & 49.600 \\
\hline 25 & 51.867 & 51.653 & 51.440 & 51.230 & 51.020 & 50.813 & 50.607 & 50.403 & 50.201 & 50.000 \\
\hline 126 & 52.282 & 52.066 & 51.852 & & 51.429 & 51.220 & .012 & 50.806 & 50.602 & 50.400 \\
\hline 27 & 52.697 & 52.479 & 52.263 & 52.049 & 51.837 & 51.626 & .417 & 51.210 & 51.004 & 50.800 \\
\hline 28 & 53.112 & 52.893 & 52.675 & 52.459 & 52.245 & 52.033 & 51.822 & 51.613 & 51.406 & 51.200 \\
\hline 29 & 53.527 & 53.306 & 53.086 & 52.869 & 52.653 & 52.439 & 52.227 & 52.026 & 52.807 & 51.600 \\
\hline 130 & 53.942 & 53.719 & 53.498 & 53.279 & 53.061 & 52 & 52.632 & 52.419 & 52.209 & 52.000 \\
\hline 32 & 54.357 & 54.132 & 53.909 & 53. & 53.469 & 53.252 & 3.036 & 52.823 & 52.610 & 52.400 \\
\hline 32 & 54.772 & 54.545 & 54. & & 878 & 53.659 & $\sqrt{42}$ & 53.226 & 53.022 & 52.800 \\
\hline 133 & 55.187 & 54.959 & 54.733 & 54.508 & 54.286 & 54.065 & 53.846 & 53.629 & 53.414 & 53.200 \\
\hline 34 & 55.602 & 55.372 & 55.144 & 54.918 & 54.694 & 54.472 & 54.251 & 54.032 & 53.025 & 53.600 \\
\hline 3 & & 55.785 & 55.556 & 55.328 & & & & & 27 & 54.000 \\
\hline 36 & 56.432 & 56.198 & 55.967 & & 55.510 & 55.285 & 55.061 & 54.839 & 54.618 & 54.400 \\
\hline 37 & 56.846 & 56.612 & 56.379 & 56.148 & 918 & & .466 & 242 & 020 & 54.800 \\
\hline 38 & 57.261 & 57.025 & 56.790 & 56.557 & 56.327 & 56.098 & 870 & 45 & 422 & 55.200 \\
\hline 39 & 57.676 & 57.438 & 202 & 56.967 & 56.735 & 56.504 & $? 75$ & 48 & & 55.600 \\
\hline & 58.091 & 57.851 & 57. & 57.377 & 57.143 & 56.911 & 80 & 56 hes & & \\
\hline & 58.506 & 58.264 & 58.025 & 57.787 & 57.551 & 57.317 & 57.085 & 56.855 & 56.627 & 56.400 \\
\hline & 58.921 & 58.678 & 58.436 & 58.197 & 57.959 & 57.724 & 57.490 & 57.258 & 57.028 & 56.800 \\
\hline & 59.336 & 59.091 & 58.848 & 58.607 & 58.367 & 58.130 & 57.895 & .661 & 57.430 & 57.200 \\
\hline & 59.751 & 59.504 & 59.259 & & 776 & & & 065 & & 57.600 \\
\hline & 60.166 & 59.917 & & & & & & & & 58.000 \\
\hline W & 60.582 & 60.331 & 60.082 & 59.836 & 59.592 & $59 \cdot 350$ & 59.109 & 58.873 & 58.635 & 58.400 \\
\hline & 60.996 & 60.744 & 60.494 & 60.246 & 60.000 & 59.756 & & & & 58.800 \\
\hline & 61.411 & 61.157 & 905 & & 60.408 & 60.163 & & 59.677 & 59.438 & 59.200 \\
\hline & & 61.570 & 61.317 & & 60.816 & 60.569 & & & & \\
\hline & 62.241 & 61.983 & 61.728 & 62.475 & 61.224 & 60.976 & & & & .000 \\
\hline
\end{tabular}





$$
241
$$

242 243

244 245 246

151

152

153

154

155

156

157

158

159

160

161

162

163

164

166

167

168

169

170

171

172

173

174

175

176

177

178

179

180

18

182

183

18

186

187

188

190

191

192

193

194

195

196

197

198

199

200
62.656

63.071

63.485

63.900

64.315

64.730

65.145

65.560

65.975

66.390

62.397

62.810

63.223

63.636

64.050

64.463

64.876

65.289

65.702

66.805

66.116

67.220

66.529

67.635

68.050

66.942

67.355

68.465

. 769

68.880

68.182

69.295

69.710

70.124

70.539

68.595

69.008

69.421

69.835

70.248

70.954

70.661

71.369

71.074

71.784

71.488

72.199

71.901

72.614

72.314

73.020

73.444

73.859

72.727

73.140

73.554

74.274

73.967

74.689

74.380

75.104

74.793

75.519

75.934

76.349

75.207

75.620

76.033

76.763

76.446

77.178

76.860

77.593

78.008

78.423

78.838

77.273

77.686

78.099

78.512

79.253

79.668

80.083

80.498

80.913

78.926

79.339

79.752

80.165

30.579

81. 328

81.743

82.158

82.573

82.983
30.992

81.405

81.818

82.231

82.645
$62.140 \quad 61.885$

$62.551 \quad 62.295$

$62.963 \quad 62.705$

$63.374 \quad 63.115$

63.786

63.525

$64.198 \quad 63.934$

64.60964 .344

65.027

65.432

65.844

64.754

65.164

65.574

66.255

66.667

65.984

67.078

67.490

67.901

66.393

66.803

67.213

67.623

$68.313 \quad 68.033$

68.724

69.136

69.547

68.443

68.852

69.262

$69.959 \quad 69.672$

70.370

70.082

70.782

71.193

70.492

70.902

71.605

72.016

71.311

71.721

72.428

72.840

73.251

73.663

72.131

72.541

72.951

74.074

74.486

74.897

75.309

75.720

76.132

74.180

74.590

75.000

75.410

75.820

76.543

76.955

76.230

77.366

77.778

78.189

76.639

77.049

77.459

77.869

$78.601 \quad 78.279$

79.012

79.424

79.835

80.247

78.689

79.093

79.508

79.918

$80.658 \quad 80.328$

81.070

80.738

81.481

81.148

81.893

81.557

82.305

81.967

$61.633 \quad 61.382$

62. 241

62.449

62.87

63.265

61.789

62.195

62.602

63.008

63.673

64.082

63.415

64.490

63.821

64.898

65.306

64.228

4. 634

65.041

65.724

65.447

66.122

66.531

66.939

65.854

66.260

66.667

67.347

67.073

67.755

68.163

68.571

68.980

67.480

67.886

68.293

69.388

68.699

69.106

69. 796

70.204

70.612

69.512

69.919 

241 83.402 83.817 84.232 84.647 85.062

205

206

207

208

209

210

211

212

213

214

85.477

85.892

86. 722

87.137

87.552

87.967

88.382

88.797

89.212

216

217

218

219

220

89.627

90.041

90.456

90.871

91.286

221

222

223

224

225

91.701

92.116

92.531

92.946

93.361

226

227
228

229

230

231

232

233

234

235

236

237

238

239

93.776

94.191

94.606

95.021

95.436

95.851

96.266

96.680

97.095

97.510

87.190

$$
87.603
$$

88.017

88.430

88.843

89.256

89.669

90.083

90.496

90.909

91.322

91.736

92.149

92.562

92.975

93.388

93.802

94.215

94.628

95.041

95.455

95.868

96.281

96.694

97.107

97.925

97.521

98.340

97.934

$98.755 \quad 98.347$

$99.170 \quad 98.760$

$99.585 \quad 99.174$

242

242

24

245

246

247

248

249

_- 100.000

243

244

245

246

247

248

249 250

86.307

$\begin{array}{lll}82.716 & 82.377 & 82.041\end{array}$

83.128

83.539

83.951

84.362

84.774

85.185

85.597

86.008

86.420

86.831

87.243

87.654

88.066

88.477

88.889

89.300

89.712

90.123

90.535

90.947

91.358

91.770

92.181

92.593

82.787

83.197

83.607

82.449

82.857

84.016

83.673

84.426

84.836

85.246

85.656

86.066

86.475

86.885

87.295

87.705

88.115

84.082

84.490

84.898

85.306

85.714

86.122

86.531

86.939

87.347

87.755

88.525

88.934

89.344

39.754

90.164

38.163

88.571

88.980

89.388

89.796

90.574

90.984

91.393

91.803

92.213

90.204

90.612

91.020

91.429

91.837

92.623

92.245

93.004

93.416

93.827

93.033

93.443

94.239

93.852

94.650

94.262

92.653

93.061

93.469

93.878

94.672

94.286

95.062

95.473

95.885

96.296

95.082

95.492

95.902

94.694

95.102

95.510

96.708

96.311

95.918

97.119

96.721

96.327

97.131

96.735

97.

942

97.541

97.143

$97.951 \quad 97.551$

98.765

98.361

97.959

98.770

98.367

99.180

99.17

$\begin{array}{cr}100.000 & 99.590 \\ \ldots & 100.000\end{array}$

98.776

99.184

99.592

..- 100.00

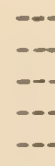

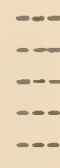

81.707

82.114

82.520

82.927

83.333

81. 377

81.781

82.186

82.591

82.996

83.740

83.401

84.146

84.553

84.959

85.366

83.806

84.211

84.615

85.020

85.772

85.425

86.179

86.585

86.992

85.830

86.235

86.640

87.398

87.045

87.805

87.449

88.211

88.618

89.024

87.854

88.259

88.664

89.431

89.069

89.837 
UNIVERSITY OF FLORIDA

31262092409282 